\title{
Fibre degradation by pig microbiota
}

Citation for published version (APA):

Long, C. (2020). Fibre degradation by pig microbiota. [Doctoral Thesis, Maastricht University].

ProefschriftMaken Maastricht. https://doi.org/10.26481/dis.20201126cl

Document status and date:

Published: 01/01/2020

DOI:

10.26481/dis.20201126cl

Document Version:

Publisher's PDF, also known as Version of record

\section{Please check the document version of this publication:}

- A submitted manuscript is the version of the article upon submission and before peer-review. There can be important differences between the submitted version and the official published version of record.

People interested in the research are advised to contact the author for the final version of the publication, or visit the DOI to the publisher's website.

- The final author version and the galley proof are versions of the publication after peer review.

- The final published version features the final layout of the paper including the volume, issue and page numbers.

Link to publication

\footnotetext{
General rights rights.

- You may freely distribute the URL identifying the publication in the public portal. please follow below link for the End User Agreement:

www.umlib.nl/taverne-license

Take down policy

If you believe that this document breaches copyright please contact us at:

repository@maastrichtuniversity.nl

providing details and we will investigate your claim.
}

Copyright and moral rights for the publications made accessible in the public portal are retained by the authors and/or other copyright owners and it is a condition of accessing publications that users recognise and abide by the legal requirements associated with these

- Users may download and print one copy of any publication from the public portal for the purpose of private study or research.

- You may not further distribute the material or use it for any profit-making activity or commercial gain

If the publication is distributed under the terms of Article $25 \mathrm{fa}$ of the Dutch Copyright Act, indicated by the "Taverne" license above, 
Fibre degradation by pig microbiota

Cheng Long 
The study was funded by the Centre for Healthy Eating \& Food Innovation (HEFI) of Maastricht University-Campus Venlo and has been made possible with the support of the Dutch Province of Limburg with a Grant to HEFI, HEFI-1venk15-25. Cheng Long received a Ph.D. scholarship from the China Scholarship Council under the Chinese Government Graduate Student Overseas Study Program (Grant No. 201603250050).

Cover-design: Cheng Long and ProefschriftMaken

Layout: Cheng Long

Printed by: ProefschriftMaken

ISBN: 978-94-6423-037-6

$\mathrm{PhD}$ thesis, Maastricht University

All rights reserced, no part of this thesis may be reproduced, distributed stored in a retrieval system, or transmitted in any form or by any means without prior permission of the author 


\title{
Fibre degradation by pig microbiota
}

\author{
Dissertation \\ to obtain the degree of Doctor at Maastrich University, \\ on the authority of the Rector Magnificus Prof.dr. Rianne M. Letschert \\ in accordance with the decision of the Board of Deans, \\ to be defended in public on Thursday $26^{\text {th }}$ of November 2020 at 16.00 hours \\ by
}

\section{Cheng Long}


Promotor

Prof. Dr. Koen Venema

\section{Co-promotor}

Dr. Sonja de Vries

Wagningen University \& Research

\section{Assessment committee:}

Prof. Dr. P. H. M. Savelkoul (chair)

Prof. Dr. K. E. Bach Knudsen, Aarhus University

Dr. A. J. M. Jansman, Wageningen University \& Research

Dr. E. E. Canfora 


\section{Abstract}

The aim of the thesis was to investigate whether degradation of rapeseed meal (RSM) by a swine gut microbiota consortium was improved by modifying RSM by treatment with cellulase (CELL), two pectinases (PECT), or alkaline (ALK) compared to untreated RSM and to assess whether microbiota composition and activity changed. The processed RSM was

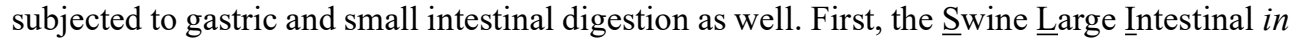
vitro Model (SLIM) was developed, which was developed based on the human, computercontrolled, dynamic TNO in vitro model of the colon, nick-named TIM-2. Second, untreated and pre-digested and processed RSM was fermented in SLIM by a standardized pig gut microbiota. A shot of $5 \mathrm{~g}$ RSM (modified or not) was directly fed to the standardized swine gut microbiota after the system adaption period, and the effect of processing methods on the RSM utilization and microbiota composition was studied. The results showed that ALK, CELL, PECT1, and PECT2 changed microbial community composition, increased the predicted abundance of microbial fibre-degrading enzymes and pathways, and increased acetic acid, propionic acid, butyric acid, and total SCFA production. The increased microbial genera positively correlated with SCFA production. Monoclonal antibody (mAb) analyses showed that the cell wall polysaccharide structures of RSM shifted after ALK, CELL, PECT1, and PECT2 treatment. The degradation of NSP during the fermentation period was dynamic, and not continuous based on the epitope recognition by mAbs. Next, a standardized swine gut microbiota was fed for $48 \mathrm{~h}$ with pre-digested RSM. Adaptive gPCA showed that CELL and ALK had larger effects on the microbiota composition than PECT1 and PECT2, and all substrates had larger effects than CON (untreated RSM). The relative abundance of family Prevotellaceae was significantly higher in CELL treatment compared to others. Prevotella 9 had significant positive correlations with propionic and valeric acid, and Mogibacterium positively correlated with acetic and caproic acid. Subsseqently, The swine gut microbiota was adapted to modified RSM in SLIM for $48 \mathrm{~h}$ first, which was followed by a shot of $5 \mathrm{~g}$ modified RSM as evaluation period. The predicted relative abundances of carbohydrate digestion and absorption, glycolysis, pentose phosphate pathway, and pyruvate metabolism were significantly increased upon CELL and ALK feeding, and CELL and ALK also exhibited increased total short-chain fatty acid (SCFA) production compared to CON. Megasphaera, Prevotella, and Desulfovibrio were significantly positively correlated with SCFA production. Findings were validated in ileal cannulated pigs, which showed that CELL and ALK increased fibre degradation of RSM. Last, a preliminary novel high-throughput technique to screen polysaccharide structures, as a tool to further study fibre degradation by

the (pig) gut microbiota, was developed. The technique was developed with the use of carbohydrate binding modules (CBMs) with unique specificities for plant cell wall polysaccharides, linked to a green fluorescent protein (GFP) as reporter. 

Abstract

Chapter 1

Chapter 2

Chapter 3

Chapter 4

Chapter 5

Chapter 6

Chapter 7

Summary

Samenvatting

总结

Impact

Acknowledgments

High-throughput screening of structures of plant cell wall polysaccharides using a novel method

General discussion

About the author
155 169

173

177

179

183

i

1

25

59

87

111

143

187 



\section{Chapter 1}

\section{General introduction}

Partly submitted for publication:

Long, C., K. Venema. Chemical and nutritional characteristics, and microbial degradation of rapeseed meal recalcitrant carbohydrates. Submitted for publication. 


\subsection{Background}

The European Union (EU) is the second-largest importer of raw material for feed (especially protein-rich feed ingredients) in the world, after China. Therefore, in order to have a more sustainable supply of responsible protein-rich ingredients, the European livestock sector needs an alternative local protein feed ingredient to fill the "protein gap".

Rapeseed meal (RSM), a byproduct from rapeseed oil production, is not only a suitable protein source for animal feed but also a potential energy source. RSM contains a high amount of cell wall polysaccharides, even higher when compared to soybean meal commonly used in the feed industry. However, the high amount of pectins in RSM can also reduce the absorption of other nutrients in the gut. Therefore, the aim of the project was to improve utilization of recalcitrant fibre of by-product of rapeseed oil. Enzymatic and chemical treatment on the RSM were performed in the project to improve the digestibility and fermentability of the by-product. This thesis deals with the pig microbiota.

As fibres are fermented in the large intestine by the gut microbiota, this thesis focused on the interaction between gut microbiota and modified RSM, and energy yield from RSM in vitro and in vivo (cannulated pigs). It was expected that our findings could help to guide swine industry with their feed additive strategies to improve efficiency and productivity, through the indication of how feed enzymes may modulate microbial status, which in trun may provide good opportunity to develop novel carbohydrase particularly in swine feed.

\subsection{Characterization of RSM carbohydrates}

Carbohydrates in rapeseed meal (B. napus) are mainly pectins and (hemi)celluloses, and carbohydrates comprise 35 to $36 \%$ of the RSM dry matter (DM) (1,2). The common feedstuff analysis shows commercial RSM contains $12.1 \%$ crude fiber and $34 \%$ nitrogen free extract (NFE) (Table 1). Carbohydrates of RSM can be categorized into non-structural carbohydrates and structural carbohydrates: a portion of the NFE is non-structural carbohydrates, whereas the crude fiber and the remainder of the NFE are structural polysaccharides. The composition of the different categories of RSM-carbohydrates are displayed in Table 1. 


\begin{tabular}{ll}
\hline Component, $\%$ & RSM \\
\hline Crude fiber & 12.1 \\
Ether extract & 3.8 to 4.1 \\
Acid detergent fiber & 17.3 \\
Neutral detergent fiber & 22.7 \\
Lignin & 2.6 \\
Non-structural carbohydrates ${ }^{\text {b }}$ & 3.2 \\
Structural carbohydrates & $29^{\text {c }}$ \\
Nitrogen free extract & 34 \\
Monosaccharides composition, mol \%o & \\
$\quad$ Glucose & 40 \\
$\quad$ Arabinose & 17 \\
$\quad$ Xalactose & 10 \\
$\quad$ Fructose & 9 \\
$\quad$ Mannose & 1 \\
$\quad$ Uhamnose & 2 \\
Water-soluble carbohydrates & 2 \\
Monosaccharides and sucrose & 20 \\
Polysaccharides & 18.7 \\
Insoluble carbohydrates & 16.7 \\
\hline Carbohydrate total & 2.0 \\
\hline
\end{tabular}

adapted from reference $(1,3-5)$

${ }^{\mathrm{b}}$ Fructose, glucose, galactose, sucrose, galactinol, raffinose and stachyose.

${ }^{\mathrm{c}}$ Pectins, cellulose residue, amylose, arabinan, arabinogalactan.

\subsubsection{Non-structural carbohydrates}

The non-structural carbohydrates in RSM are comprised of low molecular weight sugars, oligosaccharides and storage polysaccharides.

\subsubsection{Low molecular weight sugars}

Low molecular weight sugars mainly are sucrose (2.3 to $2.9 \%$ ), fructose ( 0.05 to $0.16 \%$ ), and glucose $(0.05$ to $0.16 \%)(3)$.

\subsubsection{Oligosaccharides}

The primary oligosaccharides found in RSM are raffinose, stachyose, galactinol, and myoinositol. Of those, stachyose has the highest concentration $(0.4$ to $0.5 \%)$, followed by raffinose $(0.05$ to $0.16 \%)$, galactinol $(0.1 \%)$ and myo-inositol $(0.1 \%)(6)$.

\subsubsection{Storage polysaccharides}

Only low concentrations of storage polysaccharides are present in RSM. The primary storage polysaccharide is starch. The starch content of the seed approaches $50 \%$ during early development, but starch almost completely disappears when energy stores are converted into oil as the seed matures. Rommi et al. (2) reported that starch concentrations found in the intact was the same as dehulled rapeseed press cakes $(0.2 \%$ of DM), while the starch content of canola meal was up to $2.5 \%$ of DM (7). Starch granules are comprised of two main macromolecules (amylose and amylopectin). Amylose is a linear polymer consisting of $\alpha$ 1,4-linked D-glucose units (GU), and amylose includes 500-600 GU in its native form, which can be subdivided into 1-20 chains. Amylopectin is a large branched macromolecule (molecular weight $10^{7}-10^{9} \mathrm{kDa}$ ), with approximately $5 \%$ of its total linkages consisting of $\beta$ 1,6-linked GU plus a large number of short $\alpha$-1,4-linked linear GU chains (12-70 GU) (8).

\subsubsection{Structural polysaccharides}

The rest of the RSM carbohydrates is made up of structural polysaccharides. These include 
cellulose, hemicellulose (xyloglucan and xylan), and pectic polysaccharides (homogalacturonan, rhamnogalacturonan I, arabinan, and arabinogalactan).

\subsubsection{Polysaccharides composition}

RSM contains $34.5 \%$ NSP of its DM, and RSM is high in glucosyl (40 mol\%), arabinosyl (19 $\mathrm{mol} \%)$ and uronyl residues $(18 \mathrm{~mol} \%)(1,9)$. Water-soluble and water-insoluble carbohydrates comprise 18.7 and $15.8 \%$ of the total carbohydrates content of RSM, respectively (Table 1). Water-soluble carbohydrates mainly contain glucosyl (64 mol\%) and some galactosyl residues (17 mol\%) (1), while water-insoluble carbohydrates are mainly glucosyl (32 mol\%), arabinosyl (25 mol\%), uronyl (18 mol\%) and xylosyl residues (12\%) (Table 2).

The detailed monosaccharide constituent compositions of the Water unextractable solids (WUS), which were sequentially extracted with chelating agent (ChSS) to release calciumbound pectins, dilute alkali (DASS) to release pectins tightly bound to hemicellulose, 4 and 6 molar alkali (4 MASS and 6 MASS) to release hemicelluloses, and cellulose that will remain in the residue (RES), are presented in Table 2. The composition of the glycosidic residues indicates the presence of 1,5-linked arabinan branched at O-2, galactomannan, homogalacturonan, rhamnogalacturonan I, type II arabinogalactan, glucuronoxylan, XXGGtype and XXXG-type xylo(X)glucan $(\mathrm{G})$, and cellulose in RSM $(1,2,10)$. Arabinan consists of a linear backbone of $\alpha$-L-1,5-linked arabinose (Ara) units with $\alpha$-1,2-lined or $\alpha$-1,3-lined Ara units (Figure 1). Galactomannan has a backbone of $\beta$-1,4-linked mannose units, substituted with $\alpha-1,6-$ lined galactose units (Figure 1). Homogalacturonan (HG) is the simplest form of pectin, consisting of a linear chain of $\alpha$-1,4-linked galacturonic acid, and part of its carboxyl groups is esterified with acetyl or methyl groups (Figure 1). Rhamnogalacturonan I (RG-I) consists of alternating rhamnose (Rha) and galacturonic acid (GalA) residues [-,2)- $\alpha$-L-Rha-(1,4)- $\alpha$-D-GalA- $(1,-]$, which is highly ramified with single terminal $\beta$-D-Gal and/or $\alpha$-D-Ara at position O-4 or O-3 of the rhamnosyl residues where the $\alpha$-D-GalA residues are often O-acetyl esterified at O-2 and/or O-3 (Figure 1) (11). Type II arabinogalactan (AG) is composed of a $\beta$-1,3-galactan backbone and $\beta$-1,6-galactan side chains (Figure 1). The side chains are variably decorated with L-arabinose. Glucuronoxylan (GX) has a linear backbone of $\beta$-(1-4)-linked D-xylosyl (Xyl) residues, which can be ramified with acetyl and arabinosyl residues, and some of the Xyl residues are decorated with a single $\alpha$-D-glucuronic acid (GlcA) or 4-O-methyl-D-glucuronic acid (MeGlcA) residue at $\mathrm{O} 2$ (Figure 1) $(12,13)$. Both XXGG- and XXXG-type xyloglucan (XG) exist in RSM. XXGG (Figure 1) consists of a $\beta$-1,4-linked D-glucosyl (Glc) backbone carrying various side chains of D- $\beta-1,2-G a l-D-\alpha-1,6-X y l$ and L- $\alpha-1,2-A r a-D-\beta-D-\alpha-1,6-X y l$ (14), whereas, XXXG (Figure 1) comprises a $\beta$-1,4-linked D-glucosyl backbone carrying continuous side chains of D- $\alpha-1,6-X y l, D-\beta-1,2-G a l-D-\alpha-1,6-X y 1$, and L- $\alpha-1,2-A r a-D-\beta-1,2-G a l-D-\alpha-1,6-X y l$ in every 4 residues of D- $\beta-1,4-G l c$ (14). Cellulose consists of long linear chains of $\beta-1,4$-linked DGlc residues, with a degree of polymerization between 2000-14000 residues (Figure 1). 
Table 2. Molar sugar composition of RSM fractions ${ }^{\mathrm{a}}$

\begin{tabular}{|c|c|c|c|c|c|c|c|}
\hline & \multicolumn{7}{|c|}{ Molar composition $(\mathrm{mol} \%)$} \\
\hline & Rha & Ara & Xyl & Man & Gal & Glc & UA \\
\hline RSM & 2 & 19 & 8 & 6 & 10 & 40 & 15 \\
\hline WSS & $\operatorname{Tr}^{b}$ & 7 & 1 & 7 & 17 & 64 & 5 \\
\hline WUS & 1 & 25 & 12 & 4 & 8 & 32 & 18 \\
\hline CHSS & 2 & 15 & 4 & 2 & 4 & 3 & 71 \\
\hline DASS & $\operatorname{Tr}$ & 44 & 6 & 3 & 10 & 9 & 29 \\
\hline 4MASS & $\operatorname{Tr}$ & 17 & 20 & 15 & 13 & 30 & 5 \\
\hline 6MASS & 1 & 29 & 22 & 2 & 13 & 23 & 11 \\
\hline RES & 4 & 10 & 6 & 4 & 6 & 40 & 20 \\
\hline
\end{tabular}

a adapted from reference (1)

b Trace amounts.

RSM: rapeseed meal; WSS: water soluble solids; WUS: water unextractable solids; ChSS: Chelating Agent Soluble Solids; DASS: Dilute Alkali Soluble Solids; 4 MASS: 4 Molar Alkali Soluble Solids; 6 MASS: 6 Molar Alkali Soluble Solids; RES: residue; Ara: arabinose; Xyl: xylose; Man: mannose; Fuc: fucosyl; Gal: galactose; Glc: glucose; UA: uronic acids.

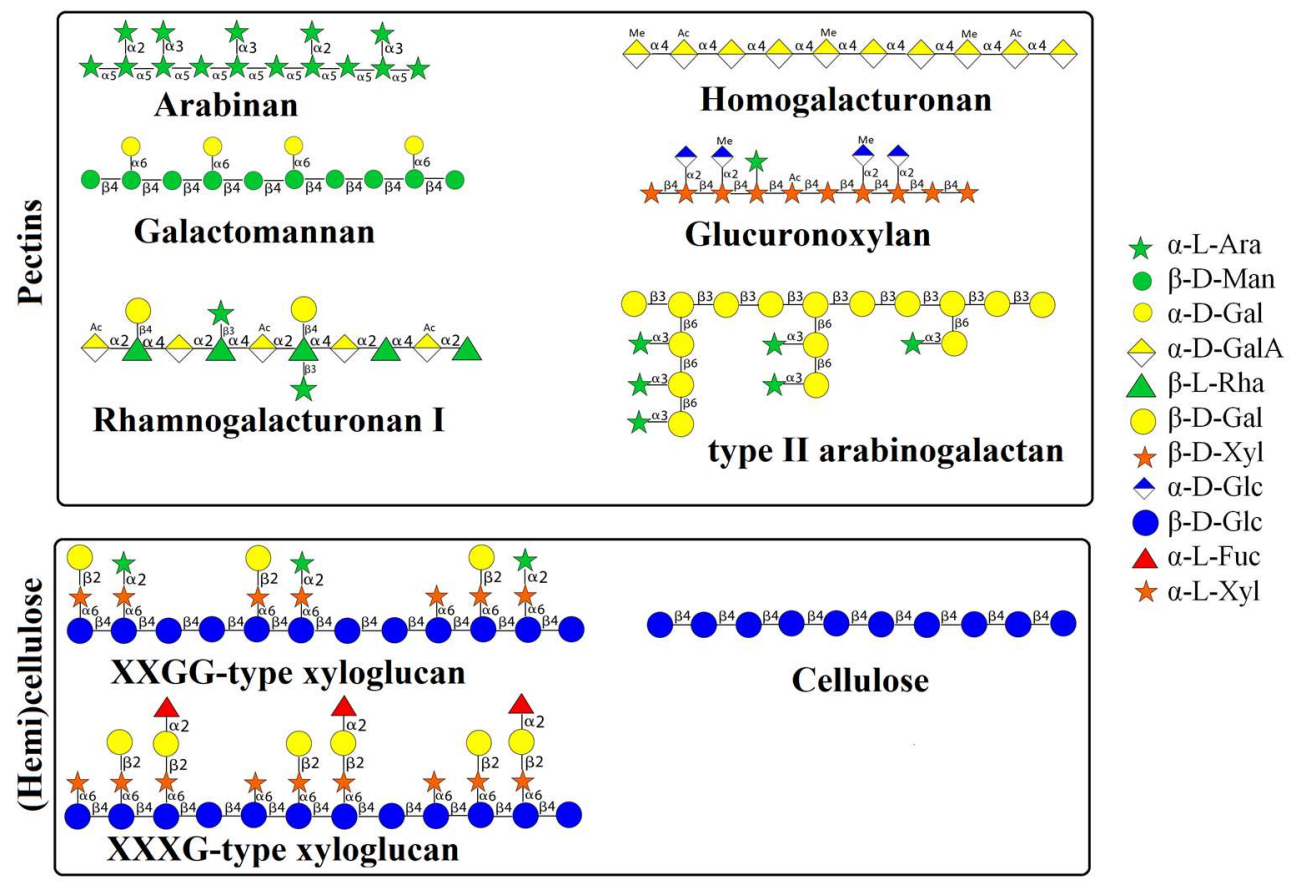

Figure 1. Schematic representation of structures of polysaccharides in RSM.

The backbone of these polysaccharides can also be esterified by methyl-esters, ethyl-esters, and glycosyls (arabinosyl, galactosyl, mannosyl, gucosyl, xylosyl, or frucosyl).

Except for containing glucosyl residues, RES (Table 2) also has quite some arabinosyl and uronyl residues $(10 \%$ and $20 \%$, respectively), which indicates that some pectic polysaccharides are tightly associated with cellulose microfibrils. This suggests that the cell wall polysaccharide matrix of rapeseed meal is strongly interlinked. The specific structures of dietary fibers of RSM are still not entirely understood. The main restriction may be the analysis method, but nowadays, the comprehensive microarray polymer profiling (CoMPP) 
technique is a powerful tool for probing cell wall structure studies (15). The profiles generated by CoMPP provide a global snapshot of cell-wall composition. It cannot only detect the amount of the particular polysaccharides but also their linkage type.

\subsubsection{Glycosidic linkage type}

A covalent link between one carbohydrate molecule and a second carbohydrate molecule is called a glycosidic bond. Glycosidic linkage is the type of bond between two adjacent glycosides in the chain of polysaccharides (16).

The proposed sugar linkage compositions of RSM fractions are shown in Table 3. The data can only be used in a qualitative manner instead of quantitative, due to the poor DMSO solubility of the fractions and the high amount of uronic acids (which are not detected with this method) present in some samples (17).

\begin{tabular}{|c|c|c|c|c|}
\hline & WUS & DASS & 4 MASS & 6 MASS \\
\hline $\mathrm{t}-\mathrm{Ara}^{\mathrm{b}}$ & 18 & 41 & 16 & 15 \\
\hline 1,2-Ara & - & 1 & 1 & - \\
\hline 1,5-Ara & 10 & 9 & 2 & 11 \\
\hline $1,2,5$-Ara & 6 & 29 & 10 & 4 \\
\hline Total Ara & 34 & 80 & 29 & 30 \\
\hline t-Xyl & 9 & 3 & 7 & 12 \\
\hline 1,2-Xyl & 2 & 1 & 10 & 3 \\
\hline 1,4-Xyl & 3 & 1 & 3 & 7 \\
\hline Total Xyl & 14 & 5 & 20 & 22 \\
\hline 1,4,6-Man & - & - & 3 & 1 \\
\hline Total Man & - & - & 3 & 1 \\
\hline t-Fuc & 2 & 1 & 2 & 5 \\
\hline 1,2,4-Fuc & 7 & 3 & - & 8 \\
\hline Total Fuc & 9 & 4 & 2 & 13 \\
\hline t-Gal & 3 & - & 4 & 6 \\
\hline 1,2-Gal & - & - & 5 & 7 \\
\hline 1,3-Gal & - & - & 7 & - \\
\hline 1,3,6-Gal & - & 7 & 4 & - \\
\hline Total Gal & 3 & 7 & 20 & 13 \\
\hline 1,4-Glc & 25 & 5 & 17 & 6 \\
\hline 1,4,6-Glc & 16 & - & 11 & 15 \\
\hline Total Glc & 41 & 5 & 28 & 21 \\
\hline $\mathrm{T} / \mathrm{B}^{\mathrm{c}}$ & 1.10 & 1.15 & 1.04 & 1.36 \\
\hline
\end{tabular}

\subsection{Microbial degradation of RSM cell wall carbohydrates}

Low molecular weight sugars and starch can be $100 \%$ digested, while (oligo- and poly-)saccharides are considered indigestible in the small intestine due to lack of the necessary enzymes in monogastric animals (18). Indeed, mammalian genomes do not encode most of the enzymes needed to degrade the structural polysaccharides present in plant material. Instead, a complex mutual dependence has developed between the mammalian host and symbiotic gut microorganisms that do possess the ability to access the abundant source of energy in carbohydrate that are indigestible by the host. The gut microbiota has glycoside hydrolases $(\mathrm{GH})$ that are able to degrade the oligo- and polysaccharides into small oligomers and monosaccharides which are subsequently taken up and fermented.

\subsubsection{Glycoside hydrolases}


Glycoside hydrolases (GHs) are a vast repertoire of cell wall-degrading enzymes that hydrolyse glycosidic bonds between two or more carbohydrate modules or a sugar and a nonsugar moiety within carbohydrates or oligosaccharides $(19,20)$. GH families widely exist in prokaryotic, eukaryotic and archaea species. A total of $167 \mathrm{GH}$ families have been identified until now (accessed on July-2020, http://www.cazy.org/Glycoside-Hydrolases.html).

Carbohydrate binding modules (CBMs) are the non-catalytic part of cell-wall-degrading enzymes, and they are attached to the GH catalytic modules. Usually, CBMs have to recognize and bind to the specific polysaccharides first, before the GHs cleave the polysaccharides (21).

\subsubsection{Arabinan}

Arabinan can be hydrolyzed by $\alpha$-L-Arabinofuranosidases (EC 3.2.1.55) and endo- $\alpha-1,5-\mathrm{L}-$ arabinanases (EC 3.2.1.99), which are found in GH families 3, 43, 51,54 and 62, and which release arabinosyl oligomers and L-arabinose (22). A previous study reported that Bacillus subtilis contained a series of arabinan-degrading genes, $a b n A, a b n 2, a b f A$, and $a b f 2$, which were induced by arabinose and arabinan, repressed by glucose, and subjected to temporal regulation (23). $A b n A$ and $a b n 2$ can express extracellular endo- $\alpha-1,5-\mathrm{L}$-arabinanases belonging to GH43, which hydrolyzes arabinan (branched) and linear $\alpha-1,5-\mathrm{L}-$ arabinan and produces arabinose and arabino-oligosaccharides (24). The resulting products, arabinose and arabino-oligosaccharides, are transported into the cell by different transport systems. Arabinose enters the cell mainly though the $\operatorname{araE}$ permease (25), and the uptake of arabinose oligomers occurs most likely via araNPQ, and ABC-type transporter (26). These products are further digested into the monosaccharide arabinose by two intracellular arabinofuranosidases, $a b f A$ and $a b f 2$, which are $\alpha$-L-arabinofuranosidases (EC 3.2.1.55) belonging to GH51. AbfA acts preferentially on $(1 \rightarrow 5)$ arabinofuranosyl linkages, and in contrast $a b f 2$ is most active on $(1 \rightarrow 2)$ and $(1 \rightarrow 3)$ linkages $(27)$. After this, L-arabinose is converted into D-xylulose-5-phosphate, which is further catabolized through the pentose phosphate pathway. The induction mechanism of these genes is mediated through negative control by the key regulator of arabinose metabolism, araR. The transcriptional repression of the $a b f A$ and $a b f 2$ genes is achieved by a tightly controlled mechanism but the regulation of $a b n A$ is more flexible.

The presence of $\alpha$-L-arabinofuranosidases (Table 4) has been determined in Bifidobacterium longum subsp. Longum (28), Bacteroides thetaiotaomicron VPI-5482 (29), Sulfolobus solfataricus P2 (30), Anoxybacillus kestanbolensis AC26Sari (31), Monoglobus pectinilyticus (32) and Roseburia faecis M72/1 (33). Endo- $\alpha-1,5-\mathrm{L}-$ arabinanases (Table 4) are present in Paenibacillus polymyxa (34), Bacillus licheniformis (35), Caldicellulorsiruptor saccharolyticus (36), Bacillus. Subtilis (37), Bacillus. Thermodenitrificans (38), Pseudomonas fluorecens subsp. cellulosa (39), Monoglobus pectinilyticus (32) and Roseburia faecis M72/1(33).

\subsubsection{Galactomannan}

Endo-1,4- $\beta$-mannanase (EC 3.2.1.78), $\beta$-mannosidase (EC 3.2.1.25) and $\alpha$-galactosidase (EC 3.2.1.22) are involved in degradation of galactomannan into monosaccharides (40). $\beta$ mannanase degrades randomly within the main chain of galactomannans, and produces shorter galactomanno-oligosaccharides that can be further hydrolyzed by $\beta$-mannosidase and $\alpha$-galactosidase. $\beta$-mannosidase hydrolyses $\beta$-1,4-linked mannose residues from the nonreducing end of the galactomanno-oligosaccharides and $\alpha$-galactosidase hydrolyses terminal $\alpha$-1,6-linked galactose residues from galactomannans or the galactomannooligosaccharides. The gut microbes Bacillus subtilis YH12 (41), Bacillus subtilis TD7 (42), 
Bacillus subtilis Bs5 (43), Bacillus licheniformis DSM13 (44), Sphingomonas sp. JB13 (45), Sphingobacterium sp. GN25 (46), Klebsiella oxytoca KUB-CW2-3 (47), Enterobacter sp. strain N18 (48), Flavobacterium sp (49), Pseudomonas cellulosa(39), Monoglobus pectinilyticus (32) and Bacteroides ovatus (50) are reported to have endo-1,4- $\beta$-mannanase (Table 4). While Bifidobacterium sp. (51), Bifidobacterium longum subsp. longum NCC2705 (52), Bacteroides ovatus (53, 54), Cellvibrio mixtus (55), Bacteroides thetaiotaomicron (56), Pseudomonas cellulose (39), Kitasatospora sp. (57) are reported to have $\beta$-mannosidase (Table 4). Moreover, Lactobacillus acidophilus NCFM (58), Lactobacillus crispatus ST1 (59), Lactobacillus brevis (60), Bifidobacterium animalis subsp. lactis Bl-04 (58), Arthrobacter sp. C2-2 (61), Bacillus megaterium (62), Dictyoglomus thermophilum (63), Bacillus stearothermophilus NCIM-5146 (64), Bacillus stearothermophilus NUB 3621(65), Bacteroides ovatus 0038-1 (66), Bifidobacterium bifidum NCIMB41171 (67), Bifidobacterium adolescentis DSM20083 (68), Bifidobacterium breve 203 (69), Clostridium stercorarium (70) and Monoglobus pectinilyticus (32) are reported to have $\alpha$-galactosidases (Table 4 ).

Asperigillus niger contains galactomannan-degradation genes, $\operatorname{agl} A, \operatorname{alg} B$ and $\operatorname{alg} C$ (encoding $\alpha$-galactosidases), and $m n d A$ (encoding a $\beta$-mannosidase). AglA and aglB have been classified into $\mathrm{GH} 27$, while $a g l C$ has been classified into $\mathrm{GH} 36$, and mndA belongs to GH2. The metabolism mechanism of galactomannan is that $\alpha$-galactosidase (encoded by aglC) and $\beta$-mannosidase (encoded by $m n d A$ ) hydrolyze the galactomannan to the oligosaccharide Gal2Man5 (monomeric sugars), where $m n d A$ cleaves single mannose units from the nonreducing end of the substrate until it reaches a galactose side-group (71), and afterward the nonreducing galactose group is hydrolyzed by $\alpha$-galactosidase (aglA, aglB, or $a g l C$ ). $A g l B$ and $m n d A$ play a major role in the degradation of galactomannan in $A$. niger. The expression of aglA is high on galactose and galactose-containing oligosaccharides but is fully repressed in the presence of glucose (72). Little is known about genes and their regulation in members of the gut microbiota.

\subsubsection{Homogalacturonan}

Homogalacturonan (HG) can be cleaved by $\alpha-1,4-\mathrm{L}$-galacturonan reducing-enddisaccharide-lyase (pectate disaccharide-lyase) (EC 4.2.2.9), (1 $\rightarrow 4)-6-O-m e t h y l-\alpha-D-$ galacturonan lyase (pectin lyase) (EC 4.2.2.10), (1 $\rightarrow 4)-\alpha$-D-galacturonan reducing-endtrisaccharide-lyase (pectate trisaccharide-lyase) (EC 4.2.2.22), $\alpha$-1,4-D-galacturonan lyase (pectate lyase) (EC 4.2.2.2), and $(1 \rightarrow 4)-\alpha$-D-galacturonan glycanohydrolase (endopolygalacturonase) (EC 3.2.1.15) (73-78). Pectin lyase (EC 4.2.2.10) provides cleavage of $\alpha$ 1,4-linked D-galacturonan methyl ester to give oligosaccharides with 4-deoxy-6-O-methyl$\alpha$-D-galact-4-enuronosyl groups at their non-reducing ends, while $\alpha$-1,4-D-galacturonan lyase (EC 4.2.2.2) cleaves $\alpha$-1.4-linked D-galacturonan to give oligosaccharides with 4deoxy- $\alpha$-D-galact-4-enuronosyl groups at their non-reducing ends. Afterwards, pectate disaccharide-lyase (EC 4.2.2.9) hydrolyzes these oligosaccharides to $(1,4-\alpha-\mathrm{D}$ galacturonosyl $)_{\mathrm{n}-2}$ and 4-(4-deoxy- $\alpha$-D-galact-4-enuronosyl)-D-galacturonate, and (1,4- $\alpha$-Dgalacturonosyl) n-2 $^{2}$ will be cleaved by pectin lyase (EC 4.2.2.10 or EC 2.2.2) again until the disaccharide. Polygalacturonase (EC 3.2.1.15) randomly hydrolyzes (1 $\rightarrow 4$ )- $\alpha$-Dgalactosiduronic linkages in pectate and other galacturonans. Pectate disaccharide-lyase (Table 4) has been reported in Caldicellulosiruptor bescii (79), Bacteroides thetaiotaomicron (80), Bacteroides ovatus (81), Bacillus pumilus BK2 (82), Eubacterium eligens, Faecalibacterium prausnitzii (83) and Monoglobus pectinilyticus (32). Pectate lyase (Table 4) has been reported in Caldicellulosiruptor bescii (79), genus Bacillus: Bacillus subtilis, B. 
licheniformis, B. cereus, B. circulans, B. pasteurii, B. amyloliquefaciens, and B. pumilus (84, 85), Paenibacillus sp. (86), Clostridium cellulovorans (87), Streptomyces thermocarboxydus (88), Bacteroides thetaiotaomicron (80), Bacteroides ovatus (81), Eubacterium eligens, Faecalibacterium prausnitzii (83) and Monoglobus pectinilyticus (32). Endopolygalacturonase (Table 4) has been reported in Caldicellulosiruptor bescii (79), Bacteroides thetaiotaomicron (80), Bacteroides ovatus (81), Eubacterium eligens, Faecalibacterium prausnitzii (83), Monoglobus pectinilyticus (32) and Bifidobacterium longum subsp. Longum (89).

\subsubsection{Rhamnogalacturonan I}

Utilization of rhamnogalacturonan I (RG-I) by microbes is mediated by a series of enzymes, which is well studied in Bacillus subtilis (90). Two main enzymes, a hydrolase and a lyase, are involved in the degradation of the RG-I backbone, whereas a few other enzymes are responsible for the breakdown of the RG-I side chains. Rhamnogalacturonan hydrolase (rhamnogalacturonan $\alpha$-L-rhamnopyranohydrolase) cleaves $\alpha-1,2$ linkages between GalA and Rha (91). Rhamnogalacturonan lyase [-L-rhamnopyranosyl-( $1 \rightarrow 4)$-alpha-Dgalactopyranosyluronate endolyase (EC 4.2.2.23)] cleaves the $\alpha-1,4$ linkages of RG-I resulting in a double bond in nonreducing GalA residue (92). Rhamnogalacturonan lyase and hydrolase (Table 4) have been reported in Caldicellulosiruptor bescii (79), Bacteroides thetaiotaomicron (80), Bacteroides ovatus (81), Bacillus subtilis (93), Bacillus licheniformis (94), Cellvibrio japonicus (95), Clostridium cellulolyticum (96), Bacillus licheniformis DSM13 (94), Pseudomonas cellulose (95), Penicillium chrysogenum (97) and Monoglobus pectinilyticus (32).

\subsubsection{Type II arabinogalactan}

Exo- $\beta$-1,3-galactanase (EC 3.2.1.145) cleaves the $\beta$-1,3-galactan backbone of type II arabinogalactan via bypassing the $\beta$-1,6-galactan side chains and releasing $\beta-1,6-$ galactooligosaccharides and their derivatives (98). The $\beta$-1,6-galactan side chains are hydrolyzed to $\beta$-1,6-galactooligosaccharides of various degrees of polymerization (DP) by endo- $\beta$-1,6-galactanase (EC 3.2.1.164) (99, 100). On the other hand, exo- $\beta-1,6-$ galactobiohydrolase releases $\beta$-1,6-galactobiose $(\beta-1,6-\mathrm{Gal} 2)$ from the nonreducing terminal end of $\beta$-1,6-galactooligosaccharides, and the $\alpha$-L-arabinofuranosidase (EC 3.2.1.55) releases arabinofuranose (Araf) from $\alpha$-1,3-Araf-substituted $\beta$-1,6-galactooligosaccharides (101). Exo- $\beta$-1,3-galactanase (Table 4) has been reported in Monoglobus pectinilyticus (32), Bifidobacterium longum subsp. longum (89), Clostridium thermocellum (102), Phanerochaete chrysosporium (103), Sphingomonas sp. (104), Bacteroides thetaiotaomicron (105), Bacteroides ovatus (105), Bacteroides caccae (106) and Bacteroides cellulosilyticus (106). Endo- $\beta$-1,6-galactanase (Table 4 ) had been reported in Streptomyces avermitilis NBRC14893 (107), Bacteroides ovatus (105), Bacteroides caccae (106), Bacteroides cellulosilyticus (106) and Bifidobacterium longum subsp. longum (89). Exo- $\beta$-1,6-galactobiohydrolase (Table 4) has been reported in Monoglobus pectinilyticus (32), Bacteroides thetaiotaomicron (106), Streptomyces avermitilis (107), Bacteroides ovatus (105), Bacteroides caccae (106), Bacteroides cellulosilyticus (106) and Bifidobacterium longum subsp. Longum (89).

\subsubsection{Glucuronoxylan}

Two enzymes, $\beta$-(1-4)-D-xylan xylanohydrolase (endo- $\beta$-1,4 xylanase) (EC 3.2.1.8) and 1,4$\beta$ xylohydrolase (exo- $\beta-1,4$ xylanase) (EC 3.2.1.37), are involved in degrading the $\beta-1,4$ xylosyl linkages in unsubstituted domains along the xylan backbone of glucuronoxylan (GX) (108, 109). Glucuronoxylanase cleaves glucuronosyl moietes which are substituted as 
monomeric side chains on the xylan backbone (110). Endo- $\beta-1,4$-xylanase (Table 4 ) has been reported in Pseudomonas boreopolis G22 (111), Bacteroides ovatus (80), Monoglobus pectinilyticus (32), Bacteroides thetaiotaomicron (105), Bacteroides caccae (106), Bacteroides cellulosilyticus (106), Clostridium thermocellum (112), Bacillus subtilis (113) and Streptomyces turgidiscabies (114). Exo- $\beta-1,4$ xylanase (Table 4 ) has been reported in Monoglobus pectinilyticus (32), Luteimicrobium xylanilyticum (115), Amycolatopsis mediterranei (115), Clostridium thermocellum (112), Bacillus subtilis (113) and Streptomyces turgidiscabies (114).

\subsubsection{Xyloglucan}

A set of glucanases and glycosidases are involved in cleaving xyloglucan (XG) into monosaccharides by two-step degradation (116, 117). Endo- $\beta$-1,4-glucanase (EC 3.2.1.4) hydrolyzes XG into large fragments, which are further degraded into monosaccharides by $\alpha$ xylosidase (EC 3.2.1.177) and $\beta$-glucosidase (EC 3.2.1.21) (118). Endo- $\beta$-1,4-glucanase (Table 4) has been reported in Monoglobus pectinilyticus (32), Caldicellulosiruptor kronotskyensis(119), Roseburia sp (33), Eubacterium rectale group (33), Ruminococcus champanellensis (120), Ruminococcus bromii (121), Ruminiclostridium cellulolyticum (122) and Phaeoacremonium minimum (123). Exo- $\beta-1,4$ xylanase (Table 4 ) has been reported in Monoglobus pectinilyticus (32), Luteimicrobium xylanilyticum (115), Amycolatopsis mediterranei (115), Clostridium thermocellum (112), Bacillus subtilis (113), Streptomyces turgidiscabies (114), Ruminiclostridium cellulolyticum (122) and Phaeoacremonium minimum (123). While $\alpha$-D-xylosidase (Table 4) has been reported in Sulfolobus solfataricus P2 (30), Talaromyces thermophilus (124), Cellvibrio japonicus (125), Bacteroides thetaiotaomicron (80), Bacteroides ovatus (80) and Monoglobus pectinilyticus (32). Moreover, $\beta$-glucosidase (Table 4) has been reported in Bifidobacterium adolescentis (126), Bacteroides ovatus (127), Listeria innocua (128), Streptomyces venezuelae (129), Pyrococcus furiosus (130), Cellvibrio japonicus (125), Caldicellulosiruptor saccharolyticus (131), Microbispora bispora (132), Thermoanaerobacter brockii (133), Thermobifida fusca (111), Pseudomonas sp. (134), Monoglobus pectinilyticus (32), Ruminococcus champanellensis (120) and Ruminococcus bromii (121)

\subsubsection{Cellulose}

The enzymes of the cellulase system consist of endo- $\beta$-1,4-glucanase (EC 3.2.1.4), exo- $\beta$ 1,4-glucanase (EC 3.2.1.91), and $\beta$-glucosidase (EC 3.2.1.21). Endo- $\beta$-1,4-glucanase and exo- $\beta$-1,4-glucanase cleave cellulose to cellodextrins and cellobiose, which are then degraded to glucose by $\beta$-glucosidase $(135,136)$. Endo and exo- $\beta$-1,4-glucanase (Table 4$)$ have been reported in Monoglobus pectinilyticus (32), Caldicellulosiruptor kronotskyensis (119), Roseburia sp (33), Eubacterium rectale group (33), Ruminococcus champanellensis (120), Ruminococcus bromii (121), Ruminiclostridium cellulolyticum (122), Paenibacillus sp (137) and Phaeoacremonium minimum (123). The enzyme $\beta$-glucosidase (Table 4) has been reported in Bifidobacterium adolescentis (126), Bacteroides ovatus (127), Listeria innocua (128), Streptomyces venezuelae (129), Pyrococcus furiosus (130), Cellvibrio japonicus (125), Caldicellulosiruptor saccharolyticus (131), Microbispora bispora (132), Thermoanaerobacter brockii (133), Thermobifida fusca (111), Pseudomonas sp. (134), Monoglobus pectinilyticus (32), Ruminococcus champanellensis (120) and Ruminococcus bromii (121). 
Table 4. Gut microbes containing the plant cell wall degrading enzymes

\begin{tabular}{|c|c|}
\hline Microbial enzymes & Bacteria \\
\hline$\alpha$-L-arabinofuranosidases & $\begin{array}{l}\text { Bifidobacterium longum subsp. Longum (28), Bacteroides thetaiotaomicron VPI- } \\
5482 \text { (29), Sulfolobus solfataricus P2 (30), Anoxybacillus kestanbolensis AC26Sari } \\
\text { (31), Monoglobus pectinilyticus (32) and Roseburia faecis M72/1 (33) }\end{array}$ \\
\hline $\begin{array}{l}\text { endo- } \alpha-1,5-\mathrm{L}- \\
\text { arabinanases }\end{array}$ & $\begin{array}{l}\text { Paenibacillus polymyxa (34), Bacillus licheniformis (35), Caldicellulorsiruptor } \\
\text { saccharolyticus (36), Bacillus. Subtilis (37), Bacillus. Thermodenitrificans (38), } \\
\text { Pseudomonas fluorecens subsp. cellulosa (39), Monoglobus pectinilyticus (32) and } \\
\text { Roseburia faecis } \mathrm{M} 72 / 1(33)\end{array}$ \\
\hline endo-1,4- $\beta$-mannanase & $\begin{array}{l}\text { Bacillus subtilis YH12 (41), Bacillus subtilis TD7 (42), Bacillus subtilis Bs5 (43), } \\
\text { Bacillus licheniformis DSM13 (44), Sphingomonas sp. JB13 (45), } \\
\text { Sphingobacterium sp. GN25 (46), Klebsiella oxytoca KUB-CW2-3 (47), } \\
\text { Enterobacter sp. strain N18 (48), Flavobacterium sp (49), Pseudomonas } \\
\text { cellulosa(39), Monoglobus pectinilyticus (32) and Bacteroides ovatus (50) }\end{array}$ \\
\hline$\beta$-mannosidase & $\begin{array}{l}\text { Bifidobacterium sp. (51), Bifidobacterium longum subsp. longum NCC2705 (52), } \\
\text { Bacteroides ovatus (53, 54), Cellvibrio mixtus (55), Bacteroides thetaiotaomicron } \\
\text { (56), Pseudomonas cellulose (39), Kitasatospora sp. (57) }\end{array}$ \\
\hline$\alpha$-Galactosidases & $\begin{array}{l}\text { Lactobacillus acidophilus NCFM (58), Lactobacillus crispatus ST1 (59), } \\
\text { Lactobacillus brevis (60), Bifidobacterium animalis subsp. lactis B1-04 (58), } \\
\text { Arthrobacter sp. C2-2 (61), Bacillus megaterium (62), Dictyoglomis } \\
\text { thermophilum (63), Bacillus stearothermophilus NCIM-5146 (64), Bacillus } \\
\text { stearothermophilus NUB 3621(65), Bacteroides ovatus 0038-1 (66), } \\
\text { Bifidobacterium bifidum NCIMB41171 (67), Bifidobacterium adolescentis } \\
\text { DSM20083 (68), Bifidobacterium breve } 203 \text { (69), Clostridium stercorarium (70) } \\
\text { and Monoglobus pectinilyticus (32) }\end{array}$ \\
\hline $\begin{array}{l}\text { Pectate disaccharide- } \\
\text { lyase }\end{array}$ & $\begin{array}{l}\text { Caldicellulosiruptor bescii (79), Bacteroides thetaiotaomicron (80), Bacteroides } \\
\text { ovatus (81), Bacillus pumilus BK2 (82), Eubacterium eligens, Faecalibacterium } \\
\text { prausnitzii (83) and Monoglobus pectinilyticus (32) }\end{array}$ \\
\hline pectate lyase & $\begin{array}{l}\text { in Caldicellulosiruptor bescii (79), genus Bacillus: Bacillus subtilis, B. B. } \\
\text { licheniformis, B. cereus, B. circulans, B. pasteurii, B. amyloliquefaciens, and B. } \\
\text { pumilus (84, 85), Paenibacillus sp. (86), Clostridium cellulovorans (87), } \\
\text { Streptomyces thermocarboxydus (88), Bacteroides thetaiotaomicron (80), } \\
\text { Bacteroides ovatus (81), Eubacterium eligens, Faecalibacterium prausnitzii (83) } \\
\text { and Monoglobus pectinilyticus (32) }\end{array}$ \\
\hline endo-polygalacturonase & $\begin{array}{l}\text { Caldicellulosiruptor bescii (79), Bacteroides thetaiotaomicron (80), Bacteroides } \\
\text { ovatus (81), Eubacterium eligens, Faecalibacterium prausnitzii (83), Monoglobus } \\
\text { pectinilyticus (32) and Bifidobacterium longum subsp. Longum (89) }\end{array}$ \\
\hline $\begin{array}{l}\text { rhamnogalacturonan } \\
\text { lyase and hydrolase }\end{array}$ & $\begin{array}{l}\text { Caldicellulosiruptor bescii (79), Bacteroides thetaiotaomicron (80), Bacteroides } \\
\text { ovatus (81), Bacillus subtilis (93), Bacillus licheniformis (94), Cellvibrio japonicus } \\
\text { (95), Clostridium cellulolyticum (96), Bacillus licheniformis DSM13 (94), } \\
\text { Pseudomonas cellulose (95), Penicillium chrysogenum (97) and Monoglobus } \\
\text { pectinilyticus (32) }\end{array}$ \\
\hline exo- $\beta$-1,3-galactanase & $\begin{array}{l}\text { Monoglobus pectinilyticus (32), Bifidobacterium longum subsp. longum (89), } \\
\text { Clostridium thermocellum (102), Phanerochaete chrysosporium (103), } \\
\text { Sphingomonas sp. (104), Bacteroides thetaiotaomicron (105), Bacteroides ovatus } \\
\text { (105), Bacteroides caccae (106) and Bacteroides cellulosilyticus (106) }\end{array}$ \\
\hline endo- $\beta$-1,6-galactanase & $\begin{array}{l}\text { Streptomyces avermitilis NBRC14893 (107), Bacteroides ovatus (105), } \\
\text { Bacteroides caccae (106), Bacteroides cellulosilyticus (106) and Bifidobacterium } \\
\text { longum subsp. longum (89) }\end{array}$ \\
\hline $\begin{array}{l}\text { exo- } \beta-1,6- \\
\text { galactobiohydrolase }\end{array}$ & $\begin{array}{l}\text { Monoglobus pectinilyticus (32), Bacteroides thetaiotaomicron (106), Streptomyces } \\
\text { avermitilis (107), Bacteroides ovatus (105), Bacteroides caccae (106), Bacteroides } \\
\text { cellulosilyticus (106) and Bifidobacterium longum subsp. longum (89) }\end{array}$ \\
\hline endo- $\beta$-1,4-xylanase & $\begin{array}{l}\text { Pseudomonas boreopolis G22 (111), Bacteroides ovatus (80), Monoglobus } \\
\text { pectinilyticus (32), Bacteroides thetaiotaomicron (105), Bacteroides caccae (106), } \\
\text { Bacteroides cellulosilyticus (106), Clostridium thermocellum (112), Bacillus } \\
\text { subtilis (113) and Streptomyces turgidiscabies (114) }\end{array}$ \\
\hline exo- $\beta-1,4$ xylanase & $\begin{array}{l}\text { Monoglobus pectinilyticus (32), Luteimicrobium xylanilyticum (115), } \\
\text { Amycolatopsis mediterranei (115), Clostridium thermocellum (112), Bacillus }\end{array}$ \\
\hline
\end{tabular}




\begin{tabular}{|l|l|}
\hline & subtilis (113) and Streptomyces turgidiscabies (114) \\
\hline $\begin{array}{l}\text { endo and exo- } \beta-1,4- \\
\text { glucanase }\end{array}$ & $\begin{array}{l}\text { Monoglobus pectinilyticus (32), Caldicellulosiruptor kronotskyensis(119), } \\
\text { Roseburia sp (33), Eubacterium rectale group (33), Ruminococcus } \\
\text { champanellensis (120), Ruminococcus bromii (121), Ruminiclostridium } \\
\text { cellulolyticum (122) and Phaeoacremonium minimum (123) }\end{array}$ \\
\hline$\alpha$-D-xylosidase & $\begin{array}{l}\text { Sulfolobus solfataricus P2 (30), Talaromyces thermophilus (124), Cellvibrio } \\
\text { japonicus }(125), \text { Bacteroides thetaiotaomicron (80), Bacteroides ovatus (80) and } \\
\text { Monoglobus pectinilyticus (32) }\end{array}$ \\
\hline$\beta$-glucosidase & $\begin{array}{l}\text { Bifidobacterium adolescentis (126), Bacteroides ovatus (127), Listeria innocua } \\
(128), \text { Streptomyces venezuelae (129), Pyrococcus furiosus (130), Cellvibrio } \\
\text { japonicus (125), Caldicellulosiruptor saccharolyticus (131), Microbispora bispora } \\
\text { (132), Thermoanaerobacter brockii (133), Thermobifida fusca (111), } \\
\text { Pseudomonas sp. (134), Monoglobus pectinilyticus (32), Ruminococcus } \\
\text { champanellensis (120) and Ruminococcus bromii (121) }\end{array}$ \\
\hline
\end{tabular}

\subsection{Processing to increase use of recalcitrant fibres}

Our previous studies have already shown that physical processing technologies cannot significantly increase fibre degradability in monogastric animals $(9,138,139)$. However, the utilization of feed enzymes is a promising method to improve fibre fermentability.

\subsubsection{Enzymatic modification}

Supplementation of cell wall degrading enzymes to improve feed efficiency for pigs gets more and more attention from the feed industry nowadays. Supplementation of cell wall degrading enzymes can remove side-chains of polysaccharides in plant cell wall, which make them more accessible to bacterial enzymes (138). In addition, cell wall degrading enzymes are able to reduce the digesta viscosity, which might affect absorption of other nutrients, by cleaving the viscous polysaccharides (e.g. pectin). In the end, carbohydrases can depolymerize polysaccharides to oligosaccharides, which have potential prebiotic effects to the host animal.

\subsubsection{Chemical modification}

A previous study showed that pre-processing with sulfuric acid could increase utilization of carbohydrates from lignocellulosic biomass (140). Mild acid-treated rye (together with heat treatment) was reported to improve the release of arabinosyl residues in chickens (141). Alkaline pretreatment of rapeseed meal in the feed improved its fermentation in pigs (142). Therefore, chemical treatment in feed might be a promising method to improve fibre fermentation in pigs.

\subsection{Swine gut microbiota}

Nowadays, it is well known that the gut microbiota plays a crucial role in the human and animal gut, which is related to the health status of the host as well as to nutrient digestibility $(143,144)$. Pigs are both an important meat source for human consumption and an animal model for biomedical research. Thus, there is numerous research about the pig gut microbiota (145-148). Ramayo-Caldas et al. (144) have studied fecal microbiota from 518 healthy piglets, and reported that the dominant microbial phyla are Firmicutes and Bacteroidetes, which is in agreement with other reports (149-152). The remaining phyla contain Actinobacteria, Proteobacteria, and others (144, 149-151). The microbial abundances in lower phylogenetic levels are highly variable, but the bacterial types are quite similar. For instance, at family level, the relative abundances of 11 families in the pig gut microbiota are Prevotellaceae (26.5\%), Veillonellaceae (18.2\%), Lachnospiraceae (16.6\%), Ruminococcaceae (16.4\%), Paraprevotellaceae (3.67\%), S24-7 (identified as Muribaculaceae, 1.91\%), Campylobacteraceae (1.24\%), Clostridiaceae(1.24\%), and others 
(146). A similar number of family types are reported in another study (152), and in addition some additional ones that ere found in that study are Streptococcaceae, Lactobacillaceae, Erysipelotrichaceae, and Coriobacteriaceae. The same findings are observed at genus level, namely that the core genera reported by different researchers are similar, but the abundances are highly variable $(146,152,153)$, e.g., due to different diet intake or diferent pig-race. Dietary fiber and resistant starch are known to modulate the intestinal microbiota and thereby contribute to gut health status by promoting the growth of beneficial microorganism in pigs and contribute to improvements in feed efficiency by production of short-chain fatty acids [SCFA] $(146,152)$. Resistant starch increased the relative abundance of Lachnospiraceae-, Prevotella-, and Rumicnococcus- affiliated phylotypes in pigs, and resulted in increases in the production of SCFA (154). Furthermore, Belobrajdic et al. (155) demonstrated that a arabinoxylan-containing diet enhanced caecal fermentation and protected colonocyte DNA against diet-induced damage in pigs. But the main drawback of these kinds of in vivo research is that the diets are not controllable. Inevitably, extra carbon sources will be introduced to the formula, which does not allow to fully explain the result mechanistically. These mechanisms could be studied in in vitro models, that allow much more control over the intervention.

\subsection{In vitro fermentation models}

It is complicated and costly to investigate the effect of feed fermentation by the gut microbiota in pigs in vivo, due to the interaction with other feed-components (such as other fibers) in the swine feed, the ethical considerations (such as using pathogens, toxic compounds, and animal experiments themselves) and other uncontrollable factors (such as infectious disease or death during the experiment) which are important for reproducibility of results. In vitro fermentative models are considered excellent alternatives to study the effect on the composition and/or activity of the gut microbiota without ethical constraints. They allow the screening of a large number of substances such as dietary ingredients and drugs, and even allow the use of radioactive compounds or pathogens. There are several fermentation models mimicking the large intestine of human beings (156-159). Nowadays, two main types of in vitro fermentation models have been developed: batch fermentation models, and dynamic fermentation models.

\subsubsection{Batch fermentation model}

Barry et al. (159) described a simple in vitro batch system allowing estimation of the fermentability of dietary fiber in the in vitro model in five European laboratories. It is a one compartment fermentation model to mimic the human colonic environment. To perform fermentation experiments in the model, small reactor vessels or test-tubes are usually used, in which different cultures, such as specific strains, intestinal or fecal microbial communities from animal or human origin, are tested for their ability to metabolize different substrates. Relying on the one compartment system (bottles), one advantage of the system is that the anaerobic conditions can be easily maintained as the system is closed and air-tight. Another distinctive characteristic of one compartment in vitro colon models is the use of a buffer with minerals as the main matrix of the medium instead of nutritive medium with additional carbon sources. The main down side of these systems however is that they are not equipped with a dialysis system, and, as a result, the microbiota should be significantly affected by the accumulation of the end products of the microbial metabolism. Thus, $\mathrm{pH}$ control and long term fermentations are not possible. These simple system are defined as "static" cultures, which are far from physiological (160). Therefore, "dynamic" fermentation models have also been developed.

\subsubsection{Dynamic fermentation model}


The most sophisticated dynamic continuous single-stage fermentation model is represented by the multiple-compartment system TIM-2 (161), and two representative three-stage fermentation models are the Simulator of the Human Intestinal Microbial Ecosystem [SHIME] (157) and SIMulator Gastro-Intestinal [SIMGI] (158), which are technically upgraded from the three-stage culture reactor of Gibson and Macfarlane in the 1980s (162, $163)$.

\subsubsection{TIM-2}

The TNO in vitro model of the colon, nick-named TIM-2, is a model that closely mimics the physiological conditions of the human proximal large intestine (Figure 1)(164), in which all parameters are computer-controlled. In brief, TIM-2 consists of four connected glass-jackets with flexible silicon membranes inside. Peristaltic movements are used to mix and move chyme through the system, by applying pressure on the flexible walls. This mixing is better than the movement in batch fermentation models and stirrers in SHIME (discussed below), where fluids and solid phases may separate. A unique design of the system is that TIM-2 is equipped with a dialysate system, a dialysis membrane running through the model and a bottle collecting the spent dialysate. This prevents accumulation of microbial metabolites, which are normally absorbed by the gut lining, and would lead to the inhibition or death of the microbes in the model when they would accumulate. The dialysis system thus maintains a highly active microbiota with a similar density as that found in the human large intestine (165). In batch fermentation models or less sophisticated models the microbiota is usually inoculated at a 100-fold or even lower density, which leads to slow growth to physiological densities. As the microbial metabolites are also taken up by the epithelial cells of the colon in vivo, TIM-2 mimics better the physiological environment in the gut. Since all metabolites are collected, an approximately $100 \%$ mass-balance can be made, which is not possible in vivo in humans. This is not even possible in animals, despite that they may be sometimes euthanized in animal trials, since part or all of the metabolites can be metabolized by colonocytes and do not reach the blood circulation, or are quickly used by tissue before (blood) sampling takes place. Therefore, TIM-2 can be used to study (molecular) mechanisms. For example, stable-isotope $\left({ }^{13} \mathrm{C}\right)$ labeled carbohydrates were fed to TIM-2 and the degradation of these carbohydrates was followed over time $(166,167)$. After incubating in TIM-2, NMR and LC-MS were used to trace the fate of these labeled substrates. TIM-2 is also highly reproducible, this has been shown in numerous studies (168-170). Of course, in vitro models have their limitations. Similar to every other in vitro model that mimics the colon, TIM-2 has no epithelial cells or immune system, and lacks feed-back mechanisms. 


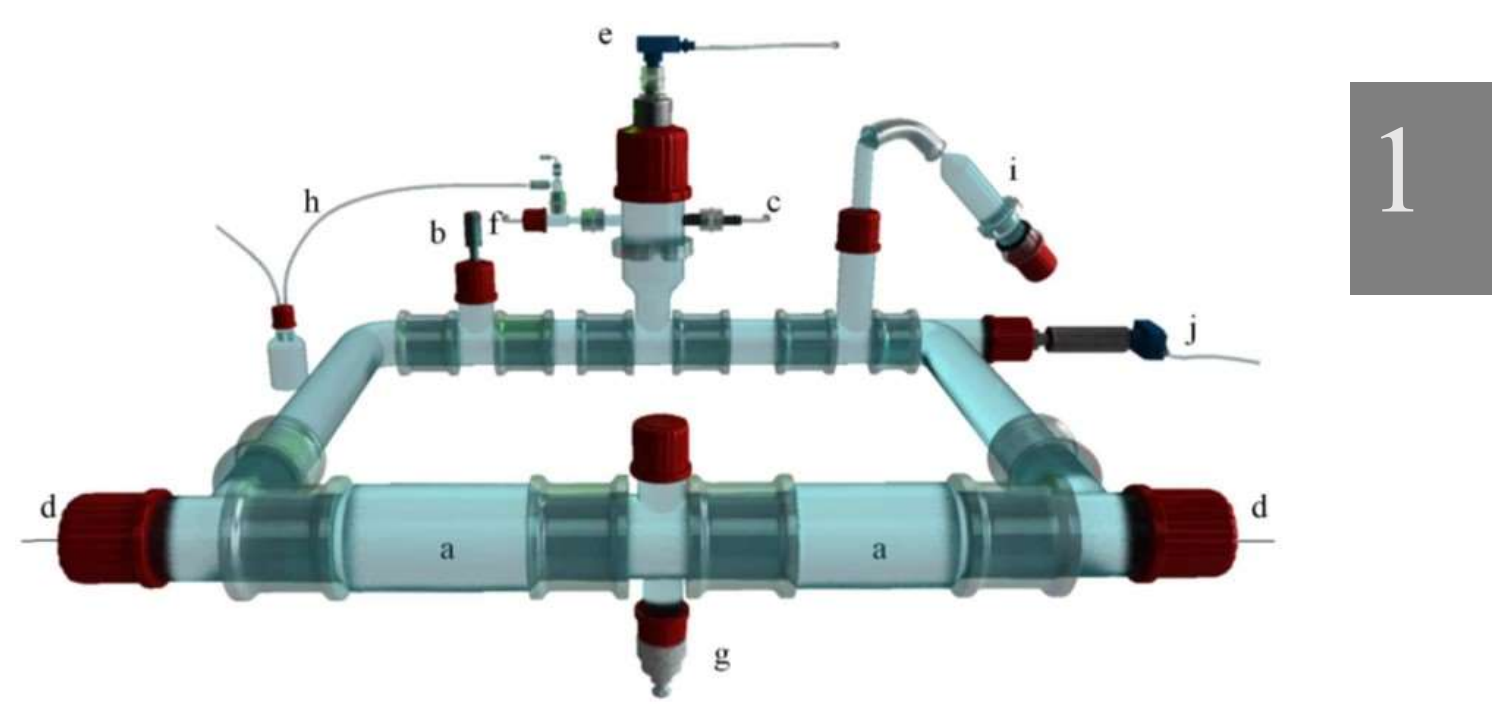

Figure 1. TIM-2 schematic. a) peristaltic compartments with a hollow fiber membrane inside, b) pH sensor, c) $\mathrm{NaOH}$ secretion, d) dialysate system, e) level sensor, f) gaseous N2 inlet, g) sampling port, h) gas outlet, i) test compound + feeding syringe, $\mathrm{j}$ ) temperature sensor.

\subsubsection{SHIME}

The Simulator of the Human Intestinal Microbial Ecosystem (SHIME) is a multicompartment dynamic simulator of the human gut (157). The conventional SHIME consists of a succession of five reactors simulating the entire gastrointestinal tract. The first two reactors, mimicking stomach and small intestine, follow the fill- and-draw principle adding a specific amount of nutritional medium, pancreatic enzymes and bile to the simulated stomach and small intestinal compartments. The remaining three compartments mimic the ascending, transverse, and descending colon, respectively (171). The colonic digestion phase is initiated by pumping the chyme of the small intestine compartment in the ascending colon vessel. Compared to TIM-2, SHIME has a few advantages. Firstly, it connects stomach and small intestine with colon together, while the TIM system consists of two separate machines (TIM-1 (156), mimicking stomach and small intestine; TIM-2 (161), mimicking the colon); secondly, recently, SHIME has been optimized to mimicking mucosal microbial colonization, and can also be coupled to a Host Microbiome Interaction module with epithelial or immune cells (172-174). However, the main drawback of SHIME is that no dialysis system is present, and as a result, SHIME cannot properly mimic the environmental conditions as discussed above. SHIME also has no feed-back mechanisms.

\subsubsection{SIMGI}

The SIMGI (SIMulator Gastro-Intestinal) is an automated gastrointestinal in vitro model, which, like SHIME, comprises five interconnected chambers that simulate the stomach, small intestine and three stages of colon (158). Since this system is quite similar to SHIME, we do not describe the model here. Details can be found in the literature (158). It looks like SHIME, but has some advantages taken from TIM-2. For example, its stomach uses peristaltic movements for mixing instead of stirring in the reactor, and the system is fully computercontrolled, which leads to more controllable $\mathrm{pH}$ and stomach emptying. No dialysis system, no feed-back mechanisms, and no host-microbe interactions are its limitations.

\subsection{Aim and outline of the thesis}

The aim of the thesis was to assess whether fermentation by swine gut microbiota of RSM, 
which was processed by enzymatic or chemical treatment, was improved compared to untreated RSM, and whether the microbiota composition and activity changed. In addition, we believed that the feeding methods had significant effects on the efficiency of fibre utilization, and therefore, the effect of different feeding methods on RSM fermentation were explored in the thesis.

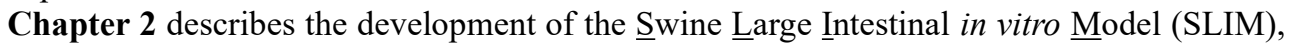
which was developed based on the human, computer-controlled, dynamic TNO in vitro model of the colon (TIM-2). The medium used for the TIM-2 (SIEM, for simulated ileum efflux medium) was optimized for the pig-microbiota (SIEMP). And pig colonic pH (5.9) and temperature $\left(39^{\circ} \mathrm{C}\right)$ were implemented. In Chapter 3, the developed SLIM-system was used to study effects of RSM on the pig microbiota. RSM was modified by enzyme (a cellulase or 2 pectinases) or chemical ( $6 \mathrm{~N}$ alkaline) treatment. A shot of $5 \mathrm{~g}$ RSM (modified or not) were directly fed to the standardized swine gut microbiota after the system adaption period, and the effect of processing methods on the RSM utilization and microbiota composition was studied. Enzyme and chemical treatment as well as degradation by the pig microbiota of the fibre was studied using a suit of monoclonal antibodies directed against carbohydrates. In Chapter 4, the modified RSM was fed to SLIM continuously for $48 \mathrm{~h}$, and investigated whether degradation of modified RSM was improved compared to untreated RSM and whether the microbiota composition was changed. In Chapter 5, the swine gut microbiota was adapted to modified RSM in SLIM for $48 \mathrm{~h}$ first, which was followed by a shot of $5 \mathrm{~g}$ modified RSM as evaluation period. Afterward, the degradability of modified RSM was validated in vivo (in cannulated pigs) by the mobile nylon bag technique. Chapter 6 describes a preliminary novel high-throughput technique to screen polysaccharide structures, as a tool to further study fibre degradation by the (pig) gut microbiota. Results of all parts of the thesis are summarized and discussed in Chapter 7. 


\section{References:}

1. Pustjens AM, Schols HA, Kabel MA, Gruppen H. Characterisation of cell wall polysaccharides from rapeseed (Brassica napus) meal. Carbohydr. Polym. 2013;98(2):1650-6.

2. Rommi K, Hakala TK, Holopainen U, Nordlund E, Poutanen K, Lantto R. Effect of enzyme-aided cell wall disintegration on protein extractability from intact and dehulled rapeseed (Brassica rapa L. and Brassica napus L.) press cakesJ. Agric. Food Chem. 2014;62(32):7989-97.

3. Bell J. Nutrients and toxicants in rapeseed meal: a review. J. Anim. Sci. 1984;58(4):996-1010.

4. Nair J, Penner GB, Yu P, Lardner H, McAllister T, Damiran D, et al. Evaluation of canola meal derived from Brassica juncea and Brassica napus seed as an energy source for feedlot steers. Can. J. Anim. Sci. 2015;95(4):599-607.

5. Rommi K, Hakala TK, Holopainen U, Nordlund E, Poutanen K, Lantto R. Effect of enzyme-aided cell wall disintegration on protein extractability from intact and dehulled rapeseed (Brassica rapa L. and Brassica napus L.) press cakesJ. Agric. Food Chem. 2014;62(32):7989-97.

6. Simbaya J, Slominski BA, Rakow G, Campbell LD, Downey RK, Bell JM. Quality characteristics of yellow-seeded Brassica seed meals: Protein, carbohydrate, and dietary fiber components. J. Agric. Food Chem. 1995;43(8):2062-6.

7. Slominski B, Campbell L, editors. The carbohydrate content of yellow-seeded canola. Proceedings of the 8th International Rapeseed Congress; 1991.

8. Gallant D, Bouchet B, Buleon A, Perez S. Physical characteristics of starch granules and susceptibility to enzymatic degradation. $\quad$ Eur. J. Clin. Nutr. 1992;46(Suppl 2):S3-S16.

9. De Vries S, Pustjens A, Kabel M, Kwakkel R, Gerrits W. Effects of processing technologies and pectolytic enzymes on degradability of nonstarch polysaccharides from rapeseed meal in broilers. Poult. Sci. 2014;93(3):589-98.

10. Ghosh P, Ghosal P, Thakur S, Lerouge P, Loutelier-Bourhis C, Driouich A, et al. Cell wall polysaccharides of Brassica campestris seed cake: isolation and structural features. Carbohydr. Polym.. 2004;57(1):7-13.

11. Colquhoun IJ, de Ruiter GA, Schols HA, Voragen AG. Identification by nmr spectroscopy of oligosaccharides obtained by treatment of the hairy regions of apple pectin with rhamnogalacturonase. Carbohydr. Resh. 1990;206(1):131-44.

12. Ebringerová A, Heinze T. Xylan and xylan derivatives-biopolymers with valuable properties, 1. Naturally occurring xylans structures, isolation procedures and properties. Macromolecular Rapid Communications. 2000;21(9):542-56.

13. Peña MJ, Zhong R, Zhou G-K, Richardson EA, O'Neill MA, Darvill AG, et al. Arabidopsis irregular xylem8 and irregular xylem9: implications for the complexity of glucuronoxylan biosynthesis. The Plant Cell. 2007;19(2):549-63.

14. Larsbrink J, Rogers TE, Hemsworth GR, McKee LS, Tauzin AS, Spadiut O, et al. A discrete genetic locus confers xyloglucan metabolism in select human gut Bacteroidetes. Nature. 2014;506(7489):498-502.

15. Kračun SK, Fangel JU, Rydahl MG, Pedersen HL, Vidal-Melgosa S, Willats WGT. Carbohydrate Microarray Technology Applied to High-Throughput Mapping of Plant Cell Wall Glycans Using Comprehensive Microarray Polymer Profiling (CoMPP). High-Throughput Glycomics and Glycoproteomics: Methods and Protocols. 2017:147-65.

16. Forsee WT, Springfield JD, Schutzbach J. Effect of phospholipids on alpha-1, 2-mannosidase activity. J. Biol. Chem. 1982;257(17):9963-7.

17. Hilz H, Bakx EJ, Schols HA, Voragen AG. Cell wall polysaccharides in black currants and bilberriescharacterisation in berries, juice, and press cake. Carbohydr. Polym.. 2005;59(4):477-88.

18. Slominski BA. Hydrolysis of galactooligosaccharides by commercial preparations of $\alpha$-galactosidase and $\beta$-fruetofuranosidase: Potential for use as dietary additives. J Sci Food Agric. 1994;65(3):323-30.

19. Flint HJ, Bayer EA. Plant cell wall breakdown by anaerobic microorganisms from the mammalian digestive tract. Ann. N. Y. Acad. Sci.. 2008;1125(1):280-8.

20. van den Brink J, de Vries RP. Fungal enzyme sets for plant polysaccharide degradation. Appl. Microbiol. Biotechnol. 2011;91(6):1477.

21. Crouch LI, Labourel A, Walton PH, Davies GJ, Gilbert HJ. The contribution of non-catalytic carbohydrate binding modules to the activity of lytic polysaccharide monooxygenases. J. Biol. Chem. 2016;291(14):7439-49.

22. Cartmell A, McKee LS, Pena MJ, Larsbrink J, Brumer H, Kaneko S, et al. The structure and function of an arabinan-specific alpha-1,2-arabinofuranosidase identified from screening the activities of bacterial GH43 glycoside hydrolases. J. Biol. Chem. 2011;286(17):15483-95.

23. Raposo MP, Inacio JM, Mota LJ, de Sa-Nogueira I. Transcriptional Regulation of Genes Encoding Arabinan-Degrading Enzymes in Bacillus subtilis. J. Bacteriol. 2004;186(5):1287-96. 
24. Leal TF, de Sa-Nogueira I. Purification, characterization and functional analysis of an endo-arabinanase (AbnA) from Bacillus subtilis. FEMS Microbiology Letters. 2004;241(1):41-8.

25. Sá-Nogueira I, Ramos SS. Cloning, functional analysis, and transcriptional regulation of the Bacillus subtilis araE gene involved in L-arabinose utilization. J. Bacteriol. 1997;179(24):7705-11.

26. Sá-Nogueira I, Nogueira TV, Soares S, de Lencastre H. The Bacillus subtilis L-arabinose (ara) operon: nucleotide sequence, genetic organization and expression. Microbiology. 1997;143(3):957-69.

27. Inacio JM, de Sa-Nogueira I. Characterization of abn2 (yxiA), encoding a Bacillus subtilis GH43 arabinanase, Abn2, and its role in arabino-polysaccharide degradation. J. Bacteriol. 2008;190(12):4272-80.

28. Komeno M, Hayamizu H, Fujita K, Ashida H. Two Novel $\alpha-<$ span class $="$ sc" $>1</$ span $>$ Arabinofuranosidases from $<\mathrm{em}>$ Bifidobacterium longum $</ \mathrm{em}>$ subsp. $<\mathrm{em}>$ longum $</ \mathrm{em}>$ Belonging to Glycoside Hydrolase Family 43 Cooperatively Degrade Arabinan. Appl. Environ. Microbiol. 2019;85(6):e0258218.

29. Mahowald MA, Rey FE, Seedorf H, Turnbaugh PJ, Fulton RS, Wollam A, et al. Characterizing a model human gut microbiota composed of members of its two dominant bacterial phyla. Proc. Natl. Acad. Sci. U. S. A. 2009;106(14):5859-64.

30. Morana A, Paris O, Maurelli L, Rossi M, Cannio R. Gene cloning and expression in Escherichia coli of a bi-functional $\beta$-D-xylosidase/ $\alpha$-L-arabinosidase from Sulfolobus solfataricus involved in xylan degradation. Extremophiles. 2007;11(1):123-32.

31. Canakci S, Kacagan M, Inan K, Belduz AO, Saha BC. Cloning, purification, and characterization of a thermostable $\alpha$-L-arabinofuranosidase from Anoxybacillus kestanbolensis AC26Sari. Appl. Microbiol. Biotechnol. 2008;81(1):61.

32. Kim CC, Healey GR, Kelly WJ, Patchett ML, Jordens Z, Tannock GW, et al. Genomic insights from Monoglobus pectinilyticus: a pectin-degrading specialist bacterium in the human colon. The ISME Journal. 2019;13(6):1437-56

33. Sheridan PO, Martin JC, Lawley TD, Browne HP, Harris HM, Bernalier-Donadille A, et al. Polysaccharide utilization loci and nutritional specialization in a dominant group of butyrate-producing human colonic Firmicutes. Microbial Genomics. 2016;2(2).

34. Wang S, Yang Y, Zhang J, Sun J, Matsukawa S, Xie J, et al. Characterization of abnZ2 (yxiA1) and abnZ3 (yxiA3) in Paenibacillus polymyxa, encoding two novel endo-1,5- $\alpha-1$-arabinanases. Bioresour. Bioprocess. 2014;1(1): 14 .

35. Seo E-S, Lim Y-R, Kim Y-S, Park C-S, Oh D-K. Characterization of a recombinant endo-1, 5- $\alpha-1-$ arabinanase from the isolated bacterium Bacillus licheniformis. Biotechnol Bioproc E. 2010;15(4):590-4.

36. Hong M-R, Park C-S, Oh D-K. Characterization of a thermostable endo-1, 5- $\alpha$-L-arabinanase from Caldicellulorsiruptor saccharolyticus. Biotechnol. Lett.. 2009;31(9):1439.

37. Inácio JM, de Sá-Nogueira I. Characterization of abn2 (yxiA), encoding a Bacillus subtilis GH43 arabinanase, Abn2, and its role in arabino-polysaccharide degradation. J. Bacteriol. 2008;190(12):4272-80.

38. Takao M, Akiyama K, Sakai T. Purification and characterization of thermostable endo-1, 5- $\alpha$-L-arabinase from a strain of Bacillus thermodenitrificans. Appl. Environ. Microbiol. 2002;68(4):1639-46.

39. Hogg D, Woo E-J, Bolam DN, McKie VA, Gilbert HJ, Pickersgill RW. Crystal structure of mannanase 26A from Pseudomonas cellulosa and analysis of residues involved in substrate binding. J Biol Chem. 2001;276(33):31186-92.

40. $\quad$ Ademark P, de Vries RP, Hägglund P, Stålbrand H, Visser J. Cloning and characterization of Aspergillus niger genes encoding an $\alpha$-galactosidase and a $\beta$-mannosidase involved in galactomannan degradation. Eur $\mathbf{J}$ Biochem. 2001;268(10):2982-90.

41. Liu H-X, Gong J-S, Li H, Lu Z-M, Li H, Qian J-Y, et al. Biochemical characterization and cloning of an endo-1, 4- $\beta$-mannanase from Bacillus subtilis YH12 with unusually broad substrate profile. Process Biochem. 2015;50(5):712-21.

42. $\quad$ Li C-y, Liu F-F, Ye J, Liu J-F, Yang S-Z, Zhang H-Z, et al. A low-temperature active endo- $\beta-1$, 4mannanase from Bacillus subtilis TD7 and its gene expression in Escherichia coli. Appl Env Biotechnol. 2018;3(2):17-25.

43. Huang JL, Bao LX, Zou HY, Che SG, Wang GX. High-level production of a cold-active B-mannanase from Bacillus subtilis Bs5 and its molecular cloning and expression. Mol Gen Microbiol Virol. 2012;27(4):147-53. 44. Songsiriritthigul C, Buranabanyat B, Haltrich D, Yamabhai M. Efficient recombinant expression and secretion of a thermostable GH26 mannan endo-1, 4- $\beta$-mannosidase from Bacillus licheniformis in Escherichia coli. Microb Cell Fact. 2010;9(1):20.

45. Zhou J, Zhang R, Gao Y, Li J, Tang X, Mu Y, et al. Novel low-temperature-active, salt-tolerant and proteases-resistant endo-1, 4- $\beta$-mannanase from a new Sphingomonas strain. J Biosci Bioeng. 2012;113(5):568-74.

46. Zhang R, Zhou J, Gao Y, Guan Y, Li J, Tang X, et al. Molecular and biochemical characterizations of a new low-temperature active mannanase. Folia Microbiol. 2015;60(6):483-92. 
47. Pongsapipatana N, Damrongteerapap P, Chantorn S, Sintuprapa W, Keawsompong S, Nitisinprasert S. Molecular cloning of kman coding for mannanase from Klebsiella oxytoca KUB-CW2-3 and its hybrid mannanase characters. Enzyme Microb Technol. 2016;89:39-51.

48. You J, Liu J-F, Yang S-Z, Mu B-Z. Low-temperature-active and salt-tolerant $\beta$-mannanase from a newly isolated Enterobacter sp. strain N18. J Biosci Bioeng. 2016;121(2):140-6.

49. Zakaria MM, Ashiuchi M, Yamamoto S, Yagi T. Optimization for $\beta$-mannanase production of a psychrophilic bacterium, Flavobacterium sp. Biosci, Biotechnol, Biochem. 1998;62(4):655-60.

50. Reddy SK, Bågenholm V, Pudlo NA, Bouraoui H, Koropatkin NM, Martens EC, et al. A $\beta$-mannan utilization locus in Bacteroides ovatus involves a GH36 $\alpha$-galactosidase active on galactomannans. FEBS Lett. 2016;590(14):2106-18.

51. Cordeiro RL, Pirolla RAS, Persinoti GF, Gozzo FC, de Giuseppe PO, Murakami MT. N-glycan Utilization by Bifidobacterium Gut Symbionts Involves a Specialist $\beta$-Mannosidase. J Mol Biol. 2019;431(4):73247.

52. Sonnenburg JL, Chen CT, Gordon JI. Genomic and metabolic studies of the impact of probiotics on a model gut symbiont and host. PLoS Biol. 2006;4(12):e413.

53. Krishnaswamyreddy S. galactmannan degradation by fungi and gut bacteria: structural enzymology and fine-tuned substrate specifcity: Lund University; 2016.

54. Bågenholm V, Reddy SK, Bouraoui H, Morrill J, Kulcinskaja E, Bahr CM, et al. Galactomannan Catabolism Conferred by a Polysaccharide Utilization Locus of Bacteroides ovatus enzyme synergy and crystal structure of a $\beta$-mannanase. J Biol Chem. 2017;292(1):229-43.

55. Dias FM, Vincent F, Pell G, Prates JA, Centeno MS, Tailford LE, et al. Insights into the molecular determinants of substrate specificity in glycoside hydrolase family 5 revealed by the crystal structure and kinetics of Cellvibrio mixtus mannosidase 5A. J Biol Chem. 2004;279(24):25517-26.

56. Tailford LE, Money VA, Smith NL, Dumon C, Davies GJ, Gilbert HJ. Mannose foraging by Bacteroides thetaiotaomicron structure and specificity of the $\beta$-mannosidase, BtMan2A. J Biol Chem. 2007;282(15):11291-9.

57. Rahmani N, Kashiwagi N, Lee J, Niimi-Nakamura S, Matsumoto H, Kahar P, et al. Mannan endo-1,4- $\beta$ mannosidase from Kitasatospora sp. isolated in Indonesia and its potential for production of mannooligosaccharides from mannan polymers. AMB Express. 2017;7(1):100.

58. Hachem MA, Fredslund F, Andersen JM, Jonsgaard Larsen R, Majumder A, Ejby M, et al. Raffinose family oligosaccharide utilisation by probiotic bacteria: insight into substrate recognition, molecular architecture and diversity of GH36 $\alpha$-galactosidases. Biocatal. Biotransformation. 2012;30(3):316-25.

59. Ojala T, Kuparinen V, Koskinen JP, Alatalo E, Holm L, Auvinen P, et al. Genome sequence of Lactobacillus crispatus ST1. J. Bacteriol. 2010;192(13):3547-8.

60. Comfort DA, Bobrov KS, Ivanen DR, Shabalin KA, Harris JM, Kulminskaya AA, et al. Biochemical analysis of Thermotoga maritima GH36 $\alpha$-galactosidase (Tm GalA) confirms the mechanistic commonality of clan GH-D glycoside hydrolases. Biochemistry. 2007;46(11):3319-30.

61. Skalova T, Dohnálek J, Spiwok V, Lipovová P, Vondráčková E, Petroková H, et al. Cold-active $\beta$ galactosidase from Arthrobacter sp. C2-2 forms compact $660 \mathrm{kDa}$ hexamers: crystal structure at $1.9 \AA$ resolution. J Mol Biol. 2005;353(2):282-94.

62. Huang Y, Zhang H, Ben P, Duan Y, Lu M, Li Z, et al. Characterization of a novel GH36 $\alpha$-galactosidase from Bacillus megaterium and its application in degradation of raffinose family oligosaccharides. Int $J$ Biol Macromol. 2018;108:98-104.

63. Schröder C, Janzer V-A, Schirrmacher G, Claren J, Antranikian G. Characterization of two novel heatactive $\alpha$-galactosidases from thermophilic bacteria. Extremophiles. 2017;21(1):85-94.

64. Gote M, Khan M, Gokhale D, Bastawde K, Khire J. Purification, characterization and substrate specificity of thermostable $\alpha$-galactosidase from Bacillus stearothermophilus (NCIM-5146). Process Biochem. 2006;41(6):1311-7.

65. Lisowska B. Genomic analysis and metabolic modelling of Geobacillus thermoglucosidasius NCIMB 11955: University of Bath; 2016.

66. Gherardini F, Babcock M, Salyers AA. Purification and characterization of two alpha-galactosidases associated with catabolism of guar gum and other alpha-galactosides by Bacteroides ovatus. J. Bacteriol. 1985;161(2):500-6.

67. Goulas T, Goulas A, Tzortzis G, Gibson GR. A novel $\alpha$-galactosidase from Bifidobacterium bifidum with transgalactosylating properties: gene molecular cloning and heterologous expression. Appl. Microbiol. Biotechnol. 2009;82(3):471-7.

68. Van Laere K, Hartemink R, Beldman G, Pitson S, Dijkema C, Schols H, et al. Transglycosidase activity of Bifidobacterium adolescentis DSM $20083 \alpha$-galactosidase. Appl. Microbiol. Biotechnol. 1999;52(5):681-8.

69. Zhao H, Lu L, Xiao M, Wang Q, Lu Y, Liu C, et al. Cloning and characterization of a novel $\alpha$ galactosidase from Bifidobacterium breve 203 capable of synthesizing Gal- $\alpha-1,4$ linkage. FEMS Microbiol Lett. 
2008;285(2):278-83

70. Kimura T, Sakka K, OHMIYA K. Cloning, sequencing, and expression of the gene encoding the Clostridium stercorarium $\alpha$-Galactosidase Aga36A in Escherichia coli. Biosci, Biotechnol, Biochem. 2003;67(10):2160-6.

71. Ademark P, Lundqvist J, Hägglund P, Tenkanen M, Torto N, Tjerneld F, et al. Hydrolytic properties of a $\beta$-mannosidase purified from Aspergillus niger. J. Biotechnol. 1999;75(2-3):281-9.

72. de Vries RP, van den Broeck HC, Dekkers E, Manzanares P, de Graaff LH, Visser J. Differential expression of three $\alpha$-galactosidase genes and a single $\beta$-galactosidase gene from Aspergillus niger. Appl. Environ. Microbiol. 1999;65(6):2453-60.

73. Edstrom R, Phaff H. Purification and Certain Properties of Pectin trans= Eliminase from Aspergihs fonsecaeus. J Biol Chem. 1963;239:2403-8.

74. Nagel CW, Vaughn RH. The degradation of oligogalacturonides by the polygalacturonase of Bacillus polymyxa. Archives of biochemistry and biophysics. 1961;94(2):328-32.

75. Nasuno S, Starr M. Polygalacturonic acid trans-eliminase of Xanthomonas campestris. Biochem J. 1967;104(1):178.

76. Mayans O, Scott M, Connerton I, Gravesen T, Benen J, Visser J, et al. Two crystal structures of pectin lyase A from Aspergillus reveal a $\mathrm{pH}$ driven conformational change and striking divergence in the substrate-binding clefts of pectin and pectate lyases. Structure. 1997;5(5):677-89.

77. Tamaru Y, Doi RH. Pectate lyase A, an enzymatic subunit of the Clostridium cellulovorans cellulosome. Proc. Natl. Acad. Sci. U.S.A.. 2001;98(7):4125-9.

78. Shevchik VE, Kester HC, Benen JA, Visser J, Robert-Baudouy J, Hugouvieux-Cotte-Pattat N. Characterization of the exopolygalacturonate lyase PelX of Erwinia chrysanthemi 3937. J. Bacteriol. 1999;181(5):1652-63.

79. Hamouda HI, Ali N, Su H, Feng J, Lu M, Li F-L. Exploration of Two Pectate Lyases from <span class="named-content genus-species" id="named-content-1" $>$ Caldicellulosiruptor bescii $</$ span $>$ Reveals that the CBM66 Module Has a Crucial Role in Pectic Biomass Degradation. Appl. Environ. Microbiol. 2020;86(16):e0078720.

80. Martens EC, Lowe EC, Chiang H, Pudlo NA, Wu M, McNulty NP, et al. Recognition and degradation of plant cell wall polysaccharides by two human gut symbionts. PLoS Biol. 2011;9(12):e1001221.

81. Luis AS, Martens EC. Interrogating gut bacterial genomes for discovery of novel carbohydrate degrading enzymes. Curr Opin Chem Biol. 2018;47:126-33.

82. Klug-Santner BG, Schnitzhofer W, Vršanská M, Weber J, Agrawal PB, Nierstrasz VA, et al. Purification and characterization of a new bioscouring pectate lyase from Bacillus pumilus BK2. J. Biotechnol. 2006;121(3):390401.

83. Lopez-Siles M, Khan TM, Duncan SH, Harmsen HJ, Garcia-Gil LJ, Flint HJ. Cultured representatives of two major phylogroups of human colonic Faecalibacterium prausnitzii can utilize pectin, uronic acids, and hostderived substrates for growth. Appl. Environ. Microbiol. 2012;78(2):420-8.

84. Hugouvieux-Cotte-Pattat N, Condemine G, Shevchik VE. Bacterial pectate lyases, structural and functional diversity. Environ Microbiol Rep. 2014;6(5):427-40.

85. Bekli S, Aktas B, Gencer D, Aslim B. Biochemical and Molecular Characterizations of a Novel pH-and Temperature-Stable Pectate Lyase from Bacillus amyloliquefaciens S6 for Industrial Application. Mol Biotechnol. 2019;61(9):681-93.

86. Yaniv O, Jindou S, Frolow F, Lamed R, Bayer EA. A simple method for determining specificity of carbohydrate-binding modules for purified and crude insoluble polysaccharide substrates. Biomass Conversion: Springer; 2012. p. 101-7.

87. Tamaru Y, Doi RH. Pectate lyase A, an enzymatic subunit of the Clostridium cellulovorans cellulosome. Proc. Natl. Acad. Sci. U.S.A.. 2001;98(7):4125-9.

88. Tonouchi A, Hara Y, Umehara R, Sanuki T, Fukusawa T, Miyairi K. Cloning of the gene encoding an endo-acting pectate lyase from Streptomyces thermocarboxydus. Biosci, Biotechnol, Biochem. 2010:0912291811-. 89. Fujita K, Sakaguchi T, Sakamoto A, Shimokawa M, Kitahara K. Bifidobacterium longum subsp. longum exo- $\beta-1,3$-galactanase, an enzyme for the degradation of type II arabinogalactan. Appl. Environ. Microbiol. 2014;80(15):4577-84.

90. Ochiai A, Itoh T, Kawamata A, Hashimoto W, Murata K. Plant Cell Wall Degradation by Saprophytic $<$ em $>$ Bacillus subtilis $<$ em> Strains: Gene Clusters Responsible for Rhamnogalacturonan Depolymerization. Appl. Environ. Microbiol. 2007;73(12):3803-13.

91. Mutter M, Beldman G, Schols HA, Voragen A. Rhamnogalacturonan [alpha]-L-Rhamnopyranohydrolase (A Novel Enzyme Specific for the Terminal Nonreducing Rhamnosyl Unit in Rhamnogalacturonan Regions of Pectin). Plant Physiol. 1994;106(1):241-50.

92. Mutter M, Colquhoun IJ, Schols HA, Beldman G, Voragen A. Rhamnogalacturonase B from Aspergillus 
aculeatus Is a Rhamnogalacturonan [alpha]-L-Rhamnopyranosyl-(1-\&gt;4)-[alpha]-D-Galactopyranosyluronide Lyase. Plant Physiol. 1996;110(1):73-7.

93. Ochiai A, Itoh T, Kawamata A, Hashimoto W, Murata K. Plant cell wall degradation by saprophytic Bacillus subtilis strains: gene clusters responsible for rhamnogalacturonan depolymerization. Appl. Environ. Microbiol. 2007;73(12):3803-13.

94. Silva IR, Larsen DM, Meyer AS, Mikkelsen JD. Identification, expression, and characterization of a novel bacterial RGI Lyase enzyme for the production of bio-functional fibers. Enzyme Microb Technol. 2011;49(2):160-6.

95. MCKIE VA, VINCKEN J-P, VORAGEN AG, VAN DEN BROEK LA, STIMSON E, GILBERT HJ. A new family of rhamnogalacturonan lyases contains an enzyme that binds to cellulose. Biochem J. 2001;355(1):16777.

96. Pagès S, Valette $\mathrm{O}$, Abdou L, Bélaïch A, Bélaïch J-P. A rhamnogalacturonan lyase in the Clostridium cellulolyticum cellulosome. J. Bacteriol. 2003;185(16):4727-33.

97. Kunishige Y, Iwai M, Nakazawa M, Ueda M, Tada T, Nishimura S, et al. Crystal structure of exorhamnogalacturonan lyase from Penicillium chrysogenum as a member of polysaccharide lyase family 26 . FEBS Lett. 2018;592(8):1378-88.

98. Fujita K, Takashi Y, Obuchi E, Kitahara K, Suganuma T. Characterization of a Novel $\beta-1-$ Arabinofuranosidase in Bifidobacterium longum functional elucidation of a DUF1680 protein family member. J Biol Chem. 2014;289(8):5240-9.

99. Aspeborg H, Coutinho PM, Wang Y, Brumer H, Henrissat B. Evolution, substrate specificity and subfamily classification of glycoside hydrolase family 5 (GH5). BMC Evol Biol. 2012;12(1):186.

100. St John FJ, González JM, Pozharski E. Consolidation of glycosyl hydrolase family 30: a dual domain 4/7 hydrolase family consisting of two structurally distinct groups. FEBS Lett. 2010;584(21):4435-41.

101. Fujita K, Sakamoto A, Kaneko S, Kotake T, Tsumuraya Y, Kitahara K. Degradative enzymes for type II arabinogalactan side chains in Bifidobacterium longum subsp. longum. Appl. Microbiol. Biotechnol. 2019;103(3):1299-310.

102. Ichinose H, Kuno A, Kotake T, Yoshida M, Sakka K, Hirabayashi J, et al. Characterization of an exo- $\beta$ 1, 3-galactanase from Clostridium thermocellum. Appl. Environ. Microbiol. 2006;72(5):3515-23.

103. Ichinose H, Yoshida M, Kotake T, Kuno A, Igarashi K, Tsumuraya Y, et al. An exo- $\beta-1$, 3-galactanase having a novel $\beta-1,3$-galactan-binding module from Phanerochaete chrysosporium. J Biol Chem. 2005;280(27):25820-9.

104. Sakamoto T, Tanaka H, Nishimura Y, Ishimaru M, Kasai N. Characterization of an exo- $\beta-1$, 3-dgalactanase from Sphingomonas sp. 24T and its application to structural analysis of larch wood arabinogalactan. Appl. Microbiol. Biotechnol. 2011;90(5):1701-10.

105. Fujita K, Sasaki Y, Kitahara K. Degradation of plant arabinogalactan proteins by intestinal bacteria: Characteristics and functions of the enzymes involved. Appl. Microbiol. Biotechnol. 2019;103(18):7451-7.

106. Cartmell A, Muñoz-Muñoz J, Briggs JA, Ndeh DA, Lowe EC, Baslé A, et al. A surface endogalactanase in Bacteroides thetaiotaomicron confers keystone status for arabinogalactan degradation. Nature microbiology. 2018;3(11):1314-26.

107. Ichinose H, Kotake T, Tsumuraya Y, Kaneko S. Characterization of an endo- $\beta$-1, 6-galactanase from Streptomyces avermitilis NBRC14893. Appl. Environ. Microbiol. 2008;74(8):2379-83.

108. Comtat J, Joseleau J-P. Mode of action of a xylanase and its significance for the structural investigation of the branched L-arabino-D-glucurono-D-xylan from redwood (Sequoia sempervirens). Carbohydr Res. 1981;95(1):101-12.

109. Takenishi S, Tsujisaka Y. On the modes of action of three xylanases produced by a strain of Aspergillus niger van Tieghem. Agric Biol Chem. 1975;39(12):2315-23.

110. Nishitani K, Nevins D. Glucuronoxylan xylanohydrolase. A unique xylanase with the requirement for appendant glucuronosyl units. J Biol Chem. 1991;266(10):6539-43.

111. Chen S, Wilson DB. Proteomic and transcriptomic analysis of extracellular proteins and mRNA levels in Thermobifida fusca grown on cellobiose and glucose. J. Bacteriol. 2007;189(17):6260-5.

112. Sharma K, Fontes CM, Najmudin S, Goyal A. Molecular organization and protein stability of the Clostridium thermocellum glucuronoxylan endo- $\beta-1,4$-xylanase of family 30 glycoside hydrolase in solution. $\mathrm{J}$ Struct Biol. 2019;206(3):335-44.

113. St. John FJ, Rice JD, Preston JF. Characterization of XynC from $<\mathrm{em}>$ Bacillus subtilis $</ \mathrm{em}>$ subsp. $<\mathrm{em}>$ subtilis $</ \mathrm{em}>$ Strain 168 and Analysis of Its Role in Depolymerization of Glucuronoxylan. J. Bacteriol. 2006;188(24):8617-26.

114. Maehara T, Yagi H, Sato T, Ohnishi-Kameyama M, Fujimoto Z, Kamino K, et al. GH30 GlucuronoxylanSpecific Xylanase from Streptomyces turgidiscabies C56. Appl. Environ. Microbiol. 2018;84(4):e01850-17.

115. Lee SH, Lee MJ, Cho H-Y, Lee JS, Rhee YH, Shin D-H, et al. Genetic and functional characterization 
of a novel GH10 endo- $\beta$-1, 4-xylanase with a ricin-type $\beta$-trefoil domain-like domain from Luteimicrobium xylanilyticum HY-24. Int J Biol Macromol. 2018;106:620-8.

116. Koyama T, Hayashi T, Kato Y, Matsuda K. Degradation of xyloglucan by wall-bound enzymes from soybean tissue I. Occurrence of xyloglucan-degrading enzymes in soybean cell wall. Plant Cell Physiol. 1981;22(7):1191-8.

117. Koyama T, Hayashi T, Kato Y, Matsuda K. Degradation of xyloglucan by wall-bound enzymes from soybean tissue II: degradation of the fragment heptasaccharide from xyloglucan and the characteristic action pattern of the $\alpha$-D-xylosidase in the enzyme system. Plant Cell Physiol. 1983;24(2):155-62.

118. Hayashi T. Xyloglucans in the primary cell wall. Annu Rev Plant Biol. 1989;40(1):139-68.

119. Conway JM, Pierce WS, Le JH, Harper GW, Wright JH, Tucker AL, et al. Multidomain, surface layerassociated glycoside hydrolases contribute to plant polysaccharide degradation by Caldicellulosiruptor species. J Biol Chem. 2016;291(13):6732-47.

120. Moraïs S, David YB, Bensoussan L, Duncan SH, Koropatkin NM, Martens EC, et al. Enzymatic profiling of cellulosomal enzymes from the human gut bacterium, R uminococcus champanellensis, reveals a fine-tuned system for cohesin-dockerin recognition. Environ Microbiol. 2016;18(2):542-56.

121. Ze X, David YB, Laverde-Gomez JA, Dassa B, Sheridan PO, Duncan SH, et al. Unique organization of extracellular amylases into amylosomes in the resistant starch-utilizing human colonic Firmicutes bacterium Ruminococcus bromii. MBio. 2015;6(5).

122. Ravachol J, De Philip P, Borne R, Mansuelle P, Maté MJ, Perret S, et al. Mechanisms involved in xyloglucan catabolism by the cellulosome-producing bacterium Ruminiclostridium cellulolyticum. Sci Rep. 2016;6(1):1-17.

123. Matsuzawa T, Kameyama A, Yaoi K. A novel isoprimeverose-producing enzyme from Phaeoacremonium minimum is active with low concentrations of xyloglucan oligosaccharides. FEBS Open Bio. 2019;9(1):92-100.

124. Guerfali M, Gargouri A, Belghith H. Catalytic properties of Talaromyces thermophilus $\alpha-1-$

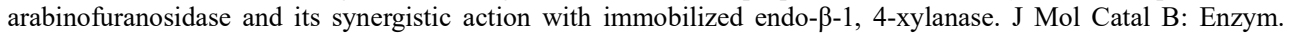
2011;68(2):192-9.

125. Larsbrink J, Izumi A, Ibatullin FM, Nakhai A, Gilbert HJ, Davies GJ, et al. Structural and enzymatic characterization of a glycoside hydrolase family $31 \alpha$-xylosidase from Cellvibrio japonicus involved in xyloglucan saccharification. Biochem J. 2011;436(3):567-80.

126. Florindo RN, Souza VP, Manzine LR, Camilo CM, Marana SR, Polikarpov I, et al. Structural and biochemical characterization of a GH3 $\beta$-glucosidase from the probiotic bacteria Bifidobacterium adolescentis. Biochimie. 2018;148:107-15.

127. Hemsworth GR, Thompson AJ, Stepper J, Sobala ŁF, Coyle T, Larsbrink J, et al. Structural dissection of a complex Bacteroides ovatus gene locus conferring xyloglucan metabolism in the human gut. Open biology. 2016;6(7):160142.

128. Nakajima M, Yoshida R, Miyanaga A, Abe K, Takahashi Y, Sugimoto N, et al. Functional and structural analysis of a $\beta$-glucosidase involved in $\beta$-1, 2-glucan metabolism in Listeria innocua. PLoS One. 2016;11(2): 0148870

129. Zmudka MW, Thoden JB, Holden HM. The structure of DesR from Streptomyces venezuelae, a $\beta$ glucosidase involved in macrolide activation. Protein Sci. 2013;22(7):883-92.

130. Cota J, Corrêa TL, Damasio AR, Diogo JA, Hoffmam ZB, Garcia W, et al. Comparative analysis of three hyperthermophilic GH1 and GH3 family members with industrial potential. N Biotechnol. 2015;32(1):13-20.

131. Hong M-R, Kim Y-S, Park C-S, Lee J-K, Kim Y-S, Oh D-K. Characterization of a recombinant $\beta$ glucosidase from the thermophilic bacterium Caldicellulosiruptor saccharolyticus. J Biosci Bioeng. 2009;108(1):3640 .

132. Wright RM, Yablonsky MD, Shalita ZP, Goyal A, Eveleigh D. Cloning, characterization, and nucleotide sequence of a gene encoding Microbispora bispora BglB, a thermostable beta-glucosidase expressed in Escherichia coli. Appl. Environ. Microbiol. 1992;58(11):3455-65.

133. Breves R, Bronnenmeier K, Wild N, Lottspeich F, Staudenbauer WL, Hofemeister J. Genes encoding two different beta-glucosidases of Thermoanaerobacter brockii are clustered in a common operon. Appl. Environ. Microbiol. 1997;63(10):3902-10.

134. McKIE VA, BLACK GW, MILLWARD-SADLER SJ, HAZLEWOOD GP, LAURIE JI, GILBERT HJ. Arabinanase A from Pseudomonas fluorescens subsp. cellulosa exhibits both an endo-and an exo-mode of action. Biochem J. 1997;323(2):547-55.

135. Béguin P, Aubert J-P. The biological degradation of cellulose. FEMS Microbiol Rev. 1994;13(1):25-58.

136. Teeri TT. Crystalline cellulose degradation: new insight into the function of cellobiohydrolases. Trends Biotechnol. 1997;15(5):160-7.

137. Adlakha N, Sawant S, Anil A, Lali A, Yazdani SS. Specific fusion of $\beta$-1, 4-endoglucanase and $\beta-1,4-$ glucosidase enhances cellulolytic activity and helps in channeling of intermediates. Appl Environ Microbiol. 
2012;78(20):7447-54.

138. de Vries S, Pustjens AM, Kabel MA, Salazar-Villanea S, Hendriks WH, Gerrits WJ. Processing technologies and cell wall degrading enzymes to improve nutritional value of dried distillers grain with solubles for animal feed: an in vitro digestion study. J. Agric. Food Chem. 2013;61(37):8821-8.

139. De Vries S, Pustjens A, Schols H, Hendriks W, Gerrits W. Improving digestive utilization of fiber-rich feedstuffs in pigs and poultry by processing and enzyme technologies: A review. Anim. Feed Sci. Technol. 2012;178(3-4):123-38.

140. Liu Z-S, Wu X-L, Kida K, Tang Y-Q. Corn stover saccharification with concentrated sulfuric acid: effects of saccharification conditions on sugar recovery and by-product generation. Bioresour Technol. 2012;119:224-33.

141. Teitge D, Campbell G, Classen H, Thacker P. Heat pretreatment as a means of improving the response to dietary pentosanase in chicks fed rye. Can. J. Anim. Sci. 1991;71(2):507-13.

142. Pustjens AM, de Vries S, Bakuwel M, Gruppen H, Gerrits WJ, Kabel MA. Degradability of rapeseed meal polysaccharides in pigs. Fiber fermentation in pigs and poultry. Sense and nonsense of its manipulation.2014; 64.

143. Lozupone CA, Stombaugh JI, Gordon JI, Jansson JK, Knight R. Diversity, stability and resilience of the human gut microbiota. Nature. 2012;489(7415):220.

144. Ramayo-Caldas Y, Mach N, Lepage P, Levenez F, Denis C, Lemonnier G, et al. Phylogenetic network analysis applied to pig gut microbiota identifies an ecosystem structure linked with growth traits. The ISME journal. 2016;10(12):2973-7.

145. Hopkins MJ, Englyst HN, Macfarlane S, Furrie E, Macfarlane GT, McBain AJ. Degradation of crosslinked and non-cross-linked arabinoxylans by the intestinal microbiota in children. Appl Environ Microbiol. 2003;69(11):6354-60.

146. Kraler M, Ghanbari M, Domig KJ, Schedle K, Kneifel W. The intestinal microbiota of piglets fed with wheat bran variants as characterised by $16 \mathrm{~S}$ rRNA next-generation amplicon sequencing. Arch Anim Nutr. 2016;70(3):173-89.

147. Warren FJ, Fukuma NM, Mikkelsen D, Flanagan BM, Williams BA, Lisle AT, et al. Food Starch Structure Impacts Gut Microbiome Composition. mSphere. 2018;3(3).

148. Martinez RC, Cardarelli HR, Borst W, Albrecht S, Schols H, Gutierrez OP, et al. Effect of galactooligosaccharides and Bifidobacterium animalis Bb-12 on growth of Lactobacillus amylovorus DSM 16698, microbial community structure, and metabolite production in an in vitro colonic model set up with human or pig microbiota. FEMS Microbiol Ecol. 2013;84(1):110-23.

149. Casellas NM, Berri EM, Fabrellas JE, Levenez F, Lemonnier G, Denis C, et al., editors. Establishment of the swine gut microbiome during early life and further impact on adult health and performance. 34 International Society for Animal Genetics Conference; 2014.

150. Costa MO, Chaban B, Harding JCS, Hill JE. Characterization of the fecal microbiota of pigs before and after inoculation with "Brachyspira hampsonii". PLoS One. 2014;9(8):e106399.

151. Cantu-Jungles TM, do Nascimento GE, Zhang X, Iacomini M, Cordeiro LM, Hamaker BR. Soluble xyloglucan generates bigger bacterial community shifts than pectic polymers during in vitro fecal fermentation. Carbohydr. Polym.. 2019;206:389-95.

152. Umu ÖC, Frank JA, Fangel JU, Oostindjer M, Da Silva CS, Bolhuis EJ, et al. Resistant starch diet induces change in the swine microbiome and a predominance of beneficial bacterial populations. Microbiome. 2015;3(1):16.

153. Niu Q, Li P, Hao S, Zhang Y, Kim SW, Li H, et al. Dynamic distribution of the gut microbiota and the relationship with apparent crude fiber digestibility and growth stages in pigs. Sci Rep. 2015;5:9938.

154. Umu OC, Frank JA, Fangel JU, Oostindjer M, da Silva CS, Bolhuis EJ, et al. Resistant starch diet induces change in the swine microbiome and a predominance of beneficial bacterial populations. Microbiome. 2015;3:16.

155. Belobrajdic DP, Bird AR, Conlon MA, Williams BA, Kang S, McSweeney CS, et al. An arabinoxylanrich fraction from wheat enhances caecal fermentation and protects colonocyte DNA against diet-induced damage in pigs. Br. J. Nutr. 2012;107(9):1274-82.

156. Minekus M, Marteau P, Havenaar R, Veld JH. A multicompartmental dynamic computer-controlled model simulating the stomach and small intestine. Atla-Altern Lab Anim. 1995;23(2):197-209.

157. Molly K, Woestyne MV, Verstraete W. Development of a 5-step multi-chamber reactor as a simulation of the human intestinal microbial ecosystem. Appl. Microbiol. Biotechnol. 1993;39(2):254-8.

158. Barroso E, Cueva C, Peláez C, Martínez-Cuesta MC, Requena T. Development of human colonic microbiota in the computer-controlled dynamic SIMulator of the GastroIntestinal tract SIMGI. Lwt-Food Sci Technol. 2015;61(2):283-9.

159. Barry J, Hoebler C, Macfarlane G, Macfarlane S, Mathers J, Reed K, et al. Estimation of the fermentability of dietary fibre in vitro: a European interlaboratory study. Br. J. Nutr. 1995;74(3):303-22.

160. Venema K, Van den Abbeele P. Experimental models of the gut microbiome. Best Pract Res $\mathrm{Cl} \mathrm{Ga.}$ 
2013;27(1):115-26.

161. Minekus M, Smeets-Peeters M, Bernalier A, Marol-Bonnin S, Havenaar R, Marteau P, et al. A computercontrolled system to simulate conditions of the large intestine with peristaltic mixing, water absorption and absorption of fermentation products. Appl Microbiol Biotechnol. 1999;53(1):108-14.

162. Gibson GR, Cummings JH, Macfarlane GT. Use of a three-stage continuous culture system to study the effect of mucin on dissimilatory sulfate reduction and methanogenesis by mixed populations of human gut bacteria. Appl Environ Microbiol. 1988;54(11):2750-5.

163. Macfarlane G, Cummings J, Macfarlane S, Gibson G. Influence of retention time on degradation of pancreatic enzymes by human colonic bacteria grown in a 3-stage continuous culture system. J. Appl. Microbiol.. 1989;67(5):521-7.

164. Venema K. The TNO in vitro model of the colon (TIM-2). The Impact of Food Bioactives on Health: Springer, Cham; 2015. p. 293-304.

165. Venema K, Nuenen Mv, Smeets-Peeters M, Minekus M, Havenaar R. TNO's in vitro large intestinal model: an excellent screening tool for functional food and pharmaceutical research. Ernährung. 2000;24(12):55864.

166. Binsl T, De Graaf A, Venema K, Heringa J, Maathuis A, De Waard P, et al. Measuring non-steady-state metabolic fluxes in starch-converting faecal microbiota in vitro. Beneficial microbes. 2010;1(4):391-405.

167. Maathuis AJ, van den Heuvel EG, Schoterman MH, Venema K. Galacto-oligosaccharides have prebiotic activity in a dynamic in vitro colon model using a (13)C-labeling technique. J Nutr. 2012;142(7):1205-12.

168. Martinez RC, Cardarelli HR, Borst W, Albrecht S, Schols H, Gutiérrez OP, et al. Effect of galactooligosaccharides and Bifidobacterium animalis Bb-12 on growth of Lactobacillus amylovorus DSM 16698, microbial community structure, and metabolite production in an in vitro colonic model set up with human or pig microbiota. FEMS Microbiol Ecol. 2013;84(1):110-23.

169. Kovatcheva-Datchary P, Egert M, Maathuis A, Rajilić-Stojanović M, De Graaf AA, Smidt H, et al. Linking phylogenetic identities of bacteria to starch fermentation in an in vitro model of the large intestine by RNAbased stable isotope probing. Environ Microbiol. 2009;11(4):914-26.

170. Rose DJ, Venema K, Keshavarzian A, Hamaker BR. Starch-entrapped microspheres show a beneficial fermentation profile and decrease in potentially harmful bacteria during in vitro fermentation in faecal microbiota obtained from patients with inflammatory bowel disease. Br. J. Nutr. 2010;103(10):1514-24.

171. Van den Abbeele P, Grootaert C, Marzorati M, Possemiers S, Verstraete W, Gérard P, et al. Microbial community development in a dynamic gut model is reproducible, colon region specific, and selective for Bacteroidetes and Clostridium cluster IX. Appl Environ Microbiol. 2010;76(15):5237-46.

172. Possemiers S, Pinheiro I, Verhelst A, Van den Abbeele P, Maignien L, Laukens D, et al. A dried yeast fermentate selectively modulates both the luminal and mucosal gut microbiota and protects against inflammation, as studied in an integrated in vitro approachJ. Agric. Food Chem. 2013;61(39):9380-92.

173. Marzorati M, Vanhoecke B, De Ryck T, Sadabad MS, Pinheiro I, Possemiers S, et al. The HMI ${ }^{\mathrm{TM}}$ module: a new tool to study the Host-Microbiota Interaction in the human gastrointestinal tract in vitro. BMC Microbiol. 2014;14(1):133.

174. Van den Abbeele P, Belzer C, Goossens M, Kleerebezem M, De Vos WM, Thas O, et al. Butyrateproducing Clostridium cluster XIVa species specifically colonize mucins in an in vitro gut model. The ISME journal. 2013;7(5):949. 


\section{Chapter 2}

\section{Polysaccharide source altered ecological network, functional profile, and short-chain fatty acid production in a porcine gut microbiota

\footnotetext{
Cheng Long ${ }^{1}$, Sonja de Vries ${ }^{2}$, Koen Venema ${ }^{{ }^{*}}$

${ }^{1}$ Faculty of Science and Engineering, Centre for Healthy Eating \& Food Innovation, Maastricht University - campus Venlo, The Netherlands

${ }^{2}$ Animal Nutrition Group, Wageningen University, Wageningen, The Netherlands

*Correspondence:

Maastricht University - campus Venlo, Centre for Healthy Eating \& Food Innovation, St. Jansweg 20, 5928 RC Venlo, The Netherlands

k.venema@maastrichtuniversity.nl; tel.: +31622435111
}

Published in Beneficial Microbes: 11 (6), 591-610. 


\begin{abstract}
Several validated dynamic in vitro models of the colon have been developed for humans, but there is no dynamic in vitro fermentation model for pigs. This study was conducted to modify the human, dynamic, computer-controlled TNO in vitro model of the colon (TIM-2) for pigs and investigate effects of different starch sources and polysaccharides on swine microbiota structure, ecological network, predictive functional profile, and short-chain fatty acids production. Our study showed that three different types of starch or two polysaccharides greatly impacted microbiota composition. Co-occurrence network analysis indicated that microbiota fed with different sources of starch changed the network topological properties. Functional profiles were predicted to vary significantly among the three starch treatments, and the original pig fecal inoculum was more similar to maize starch treatment. On the other hand, compared with maize starch and arabinoxylans (AX), the microbial composition of the original inoculum was more similar when AX-XG (arabinoxylans and xyloglucan) were added, and the functional profile of the original inoculum also clustered with AX-XG. The cumulative production of acetic, propionic, and butyric acid on maize starch were significant higher than those on potato starch and wheat starch, while only the amount of acetic acid was significant higher on $\mathrm{AX}-\mathrm{XG}$ than that on $\mathrm{AX}$. In conclusion, supplementation of maize starch as the starch source together with $\mathrm{AX}$ and $\mathrm{XG}$, leads to the bacteria being more stable in the in vitro model and closer to the original inoculum and microbial function compared to potato starch, wheat starch and AX. A maize basal diet may improve energy absorption in the large intestine in growing pigs.
\end{abstract}


Introduction

Nowadays, it is well known that the gut microbiota plays a crucial role in the human and animal gut, which is related to the health status of the host and also to nutrient digestibility(1, 2). Pigs are both an important meat source for human consumption and an animal model for biomedical research. Thus, there is numerous research about pig gut microbiota (3-6). Ramayo-Caldas et al.(1) have studied the fecal microbiota from 518 healthy piglets, and reported that the dominant microbial phyla are Firmicutes and Bacteroidetes, which are in agreement with other reports (7-10). The remaining phyla contain Actinobacteria, Proteobacteria, and others (1, 7-9). The microbial abundances in lower phylogenetic levels are hightly variable, but the bacterial types are quite similar. For instance, at family level, 11 families in pig gut microbiota are reported: Prevotellaceae, Veillonellaceae, Lachnospiraceae, Ruminococcaceae, Paraprevotellaceae, S24-7 (identified as Muribaculaceae), Campylobacteraceae, Clostridiaceae, and others (3). A similar amount of family types are reported in another study (10), and some additional ones are Streptococcaceae, Lactobacillaceae, Erysipelotrichaceae, and Coriobacteriaceae. The same findings are observed at genus level, namely that the core genera reported by different researchers are similar, but the abundances are highly variable $(3,10,11)$, e.g., due to different diet intake. Dietary fiber and resistant starch are known to modulate the intestinal microbiota and thereby contribute to gut health status by promoting the growth of beneficial microorganism in pigs and contribute to improvements in feed efficiency by production of short chain fatty acids [SCFA] $(3,10)$. Resistant starch increased the relative abundance of Lachnospiraceae-, Prevotella-, and Rumicnococcus- affiliated phylotypes in pigs as a result of increases in the production of SCFA (10). Furthermore, Belobrajdic et al. (12) demonstrated that arabinoxylan-diet enhanced caecal fermentation and protected colonocyte DNA against diet-induced damage in pigs. But the main drawback of these kinds of in vivo research is that the diets are not controllable. Inevitably, extra carbon sources will be introduced to the formula, which does not allow to fully explain the result mechanistically. It is complicated and costly to investigate the effect of feed fermentation by the gut microbiota in pigs in vivo, due to the interaction with other feed-components (such as other fibers) in the swine feed, the ethical considerations (such as using pathogens, toxic compounds, and animal experiments itself) and other uncontrollable factors (such as infectious disease or death during the experiment, which are important for reproducibility of results). In vitro fermentative models are considered excellent alternatives to study the effect on the composition and/or activity of the gut microbiota without ethical constraints. They allow the screening of a large number of substances such as dietary ingredients and drugs, and even allow the use of radioactive compounds or pathogens. There are several fermentation models mimicking the large intestine of human beings (13-16).

Nowadays, two main types of in vitro fermentation models have been developed: batch fermentation models, and dynamic fermentation models. Barry et al. (16) described a simple in vitro batch system, which is a one compartment fermentation model to mimic human colonic environment. The main side effect of these systems however is that they are not equipped with a dialysis system, and, as a result, the microbiota should be significantly affected by the accumulation of the end products of the microbial metabolism, such as SCFA. Thus, $\mathrm{pH}$ control and long term fermentations are not possible. These simple system are defined as "static" cultures, which are far from physiological conditions (17). Therefore, dynamic fermentation models have also been developed. The most sophisticated dynamic continuous single-stage fermentation model is represented by the multiple-compartment 
system TIM-2 (18), and two representative three-stage fermentation models are the Simulator of the Human Intestinal Microbial Ecosystem [SHIME] (14) and SIMulator Gastro-Intestinal [SIMGI] (19), which are technically upgraded from the three-stage culture reactor of Gibson and Macfarlane in the 1980s $(20,21)$.

The TNO in vitro model of the colon, nick-named TIM-2, is a model that closely mimics the physiological conditions of the human proximal large intestine [Figure S1] (18), in which all parameters are computer-controlled (elaborated in the Materials and Methods section). In brief, TIM-2 consists of four connected glass-jackets with flexible silicon membranes inside. Peristaltic movements are used to mix and move chyme through the system, by applying pressure on the flexible walls. This mixing is better than the stirring in batch fermentation models or other in vitro models, where fluids and solid phases separate. A unique design of the system is that TIM-2 is equipped with a dialysate system, a dialysis membrane running through the model and a bottle collecting the spent dialysate (Figure S1). This prevents accumulation of microbial metabolites, which would lead to the inhibition or death of the microbes in the model when they would accumulate. The dialysis system thus maintains a highly active microbiota with a similar density as that found in the human large intestine (22). In batch fermentation models or less sophisticated models the microbiota is usually inoculated at a 100-fold or even lower density, which leads to slow growth to physiological densities. As the microbial metabolites are also taken up by the epithelial cells of the colon in vivo, TIM-2 mimics better the physiological environment in the gut. Since all metabolites are collected, an approximately $100 \%$ mass-balance can be made, which is not possible in vivo. This is not even possible in animals, despite that they may be sometimes euthanized in animal trials, since part or all of the metabolites can be metabolized by colonocytes and cannot reach the blood circulation, or are quickly used by tissue before sampling takes place. Therefore, TIM-2 can be used to study (molecular) mechanisms. For example, stable-isotope $\left({ }^{13} \mathrm{C}\right)$ labeled carbohydrates were fed to TIM-2 and the degradation of these carbohydrates was followed over time $(23,24)$. After incubating in TIM-2, NMR and LC-MS were used to trace the fate of these labeled substrates. TIM-2 is also highly reproducible, this has been shown in numerous studies $(5,25,26)$. Of course, in vitro models have their limitations. Similar to every other in vitro model that mimics the colon, TIM-2 has no epithelial cells or immune system, and lacks feed-back mechanism.

The Simulator of the Human Intestinal Microbial Ecosystem (SHIME) and SIMGI (SIMulator Gastro-Intestinal) are multi-compartment dynamic simulators of the human gut $(14,15)$. Compared to TIM-2, SHIME and SIMGI have their own advantages. For instance, they connect stomach and small intestine with colon together, while the TIM system consists of two separate machines [TIM-1 (13), mimicking stomach and small intestine; TIM-2 (18), mimicking the colon]. However, the main drawback of SHIME and SIMGI is that no dialysis system is present, and as a result, they cannot properly mimic the environmental conditions as discussed above. They also have no feed-back mechanism.

Mostly, these systems have been used to study the human microbiota. To our knowledge, advanced and dynamic in vitro models for swine studies have not been developed, and TIM2 has its own unique advantages compared with other in vitro models. Therefore in this study, the authors intended on changing the parameters of TIM-2, which is mimicking the colonic physiological conditions of human beings, to the parameters of the swine large intestine, such as $\mathrm{pH}$, temperature, and the growth medium. The growth medium for the gut microbiota was initially developed by (20) which was subsequently used for TIM-2 (standard ileum effluent medium, SIEM), SHIME, SIMGI, and other models in the field with minor modifications. 
The medium comprises a source of protein and complex carbohydrates (i.e. resistant starch, pectin, arabinogalactan, xylan) that are not digested by the human enzymes. In the current study, the medium was optimized for use by the swine gut microbiota, as the strategy of modifying TIM-2 to swine in vitro large intestinal model (named SLIM). Based on this, two studies were designed: Study I, to assess the effect of different starch sources (potato, wheat, and maize starch) on pig microbiota in SLIM (in order to define the optimized starch source to supplement to standard ileum effluent medium for pigs, SIEMP); Study II, after selecting the optimal starch in the SIEM recipe, two more polysaccharides (xyloglucan and arabinoxylan) were added to the recipe of SIEM to investigate their effect on pig microbiota (to optimize SIEMP). Study I was trying to clarify which starch source fits pig microbiota in vitro best. Study II was designed to best mimic the polysaccharide composition of swine basal diets to allow growth of most of the members of the pig microbiota. 
1 Materials and Methods

\subsection{The Swine in vitro large intestinal model}

The Swine in vitro Large Intestinal Model (SLIM) (Figure S1) was established on the basis of the human, computer-controlled, dynamic TNO in vitro model of the colon, TIM-2 (18), which simulates the physiological conditions in the lumen of the human proximal colon. In brief, the units, consisting of 4 interlinked glass compartments with a flexible membrane inside (Figure S1a), were flushed with nitrogen (Figure S1f) prior to the introduction of the inoculum, and throughout the remainder of the experiment. The volume of the lumen is 120 $\mathrm{mL}$.

The system was maintained under the condition of porcine body temperature $\left(39^{\circ} \mathrm{C}\right)$ using a temperature sensor (Figure S1j; Easytem R31, Endress + Hauser, Nesselwang, Germany) for $96 \mathrm{~h}$ with the $\mathrm{pH}$ kept at or above 5.9 by automatic titration with $2 \mathrm{M} \mathrm{NaOH}$ (Figure S1c). In order to prevent the accumulation of microbial metabolites, which would otherwise inhibit and/or kill the microbiota in a matter of 5-6 hours, water and microbial metabolites were removed from the lumen using a dialysate system (Figure S1d; described below) consisting of a semi-permeable hollow membrane which runs through the lumen. For all the experiments, the speed of the dialysis fluid was set at $1.5 \mathrm{ml} \mathrm{min}^{-1}$ (27). Mixing and transport of the intestinal contents occurs through the simulation of peristaltic movements, by squeezing of the flexible membrane. The system was inoculated with a standardized, complex, high density, metabolically active, anaerobic microbiota of swine origin.

\subsection{Feces collection and standardization}

Grab samples, fresh and not contaminated with urine, were collected from the floor from growing pigs (48 pens with 6 pigs/pen, Hypor Libra x Hypor Maxter, Hendrix Genetics, Boxmeer, the Netherlands), and were put into a bag and placed immediately into boxes with gastight lids, containing an Anaerocult ${ }^{\circledR}$ strip (AnaeroGenTM, Cambridge, UK) to create anaerobic conditions during transport to the anaerobic cabinet. This transport never lasted longer than 3 hours. The pigs received no antibiotics and their body weight was approximately $70 \mathrm{~kg}$. Pigs were fed ad libitum with a commercial diet, which was formulated to meet or exceed the nutrient requirements of growing pigs according to CVB (Dutch research institute for livestock feed and nutrition). Since this was not an intervention study, feces collection from the floor of the pins of the pigs did not require ethical approval in accordance with local/national guidelines.

The standardized pig microbiota used to inoculate in the SLIM system was prepared in an anaerobic cabinet [Sheldon Lab - Bactron IV, Gomelius, OR, USA] (22). The fecal samples were weighed and mixed with an equal weight of dialysate solution (described below). Then, glycerol was added to final concentration of $15 \%$ and the slurry was mixed well. The fecal material was aliquoted $(45 \mathrm{ml})$ in $50 \mathrm{ml}$ tubes, snap-frozen in liquid nitrogen and stored at $80{ }^{\circ} \mathrm{C}$.

\subsection{Study I: The effect of different starch sources on pig microbiota 1.3.1 Experimental setup}

To start the experiment, SLIM was inoculated with $60 \mathrm{ml}$ of the standardized microbiota (described above) plus $60 \mathrm{ml}$ of dialysis liquid (described below) yielding a total of $120 \mathrm{ml}$ (total volume of the system). The microbiota was fed with simulated ileal effluent medium (described below), but the original starch source as described by (18) was replaced by potato $\left(4.5 \mathrm{~g} \mathrm{~d}^{-1}\right)$, wheat $\left(4.5 \mathrm{~g} \mathrm{~d}^{-1}\right)$, or maize starch $\left(4.5 \mathrm{~g} \mathrm{~d}^{-1}\right)$.

\subsubsection{Simulated ileal effluent medium and dialysate}

The simulated ileal effluent medium (SIEM), slightly modified from (20) and described in 
(27) together with the dialysate composition, contained the following components $\left(\mathrm{g} \mathrm{L}^{-1}\right)$ : 74.6 starch (potato, wheat or maize), 9.0 xylan, 9.0 pectin, 9.0 amylopectin,9.0 arabinogalactan, 31.5 Tween $80,43.7$ casein, 0.7 ox-bile, 43.7 bactopepton, 4.7 $\mathrm{K}_{2} \mathrm{HPO}_{4} .3 \mathrm{H}_{2} \mathrm{O}, 0.009 \mathrm{FeSO}_{4} .7 \mathrm{H}_{2} \mathrm{O}, 8.4 \mathrm{NaCl}, 0.8 \mathrm{CaCl}_{2} .2 \mathrm{H}_{2} \mathrm{O}, 0.7 \mathrm{MgSO}_{4} .7 \mathrm{H}_{2} \mathrm{O}, 0.05$ bile, 0.02 haemin and 0.3 cysteine $\mathrm{HCl}$, plus $1.5 \mathrm{~mL}$ of a vitamin mixture containing (per litre): 1 $\mathrm{mg}$ menadione, $0.5 \mathrm{mg}$ vitamin B12, $2 \mathrm{mg}$ D-biotin, $10 \mathrm{mg}$ pantothenate, $5 \mathrm{mg}$ aminobenzoic, $4 \mathrm{mg}$ thiamine and $5 \mathrm{mg}$ nicotinamide acid. The $\mathrm{pH}$ was adjusted to 5.9.

Dialysis liquid contained (per litre): $2.5 \mathrm{~g} \mathrm{~K}_{2} \mathrm{HPO}_{4} .3 \mathrm{H}_{2} \mathrm{O}, 0.005 \mathrm{~g} \mathrm{FeSO}_{4} .7 \mathrm{H}_{2} \mathrm{O}, 4.5 \mathrm{~g} \mathrm{NaCl}$, $0.45 \mathrm{~g} \mathrm{CaCl}_{2} .2 \mathrm{H}_{2} \mathrm{O}, 0.05 \mathrm{~g}$ bile, $0.5 \mathrm{~g} \mathrm{MgSO}_{4} .7 \mathrm{H} 2 \mathrm{O}$ and $0.4 \mathrm{~g}$ cysteine $\cdot \mathrm{HCl}$, plus $1 \mathrm{~mL}$ of the vitamin mixture. All medium components were purchased at Tritium Microbiology (Eindhoven, The Netherlands). SIEM only contains indigestible carbohydrates and hence did not require pre-digestion. SIEM was administered at $60 \mathrm{ml} \mathrm{d}^{-1}$ [corresponding to $7.5 \mathrm{~g}$ carbohydrate $\left.\mathrm{d}^{-1}\right](28)$.

\subsection{Study II: The effect of polysaccharides on pig microbiota}

Except for SIEM all other procedures were the same as for the above experiment. Starch of SIEM was changed to the optimal source discovered in the above experiment (maize). Moreover, arabinoxylan (AX, $0.54 \mathrm{~g} \mathrm{~d}^{-1}$; BioActor, Maastricht, the Netherlands), or arabinoxylan $\left(0.54 \mathrm{~g} \mathrm{~d}^{-1}\right)$ and xyloglucan $\left(0.54 \mathrm{~g} \mathrm{~d}^{-1}\right.$; CarboMer, Inc., San Diego, CA USA) together $(\mathrm{AX}-\mathrm{XG})$ were added to the SIEM recipe.

\subsection{Sample collection and statistics}

Four SLIM-units were run in parallel each time. Experiments were performed in duplicate (n $=2$ ). Each starch source (Study I) or non-starch polysaccharides (Study II) was run in two units, and samples of each unit were taken from the lumen and dialysate after 24, 48 and 72 hours to analyze microbiota composition and SCFA production. Samples were snap-frozen in liquid nitrogen and stored until analyses (described below). After 24 and $48 \mathrm{~h}$ of fermentation $25 \mathrm{ml}$ of lumen sample was removed from the system to mimic the transit of chyme from the proximal to the distal colon (27).

\subsubsection{Microbial DNA extraction and sequencing of the V3-V4 region of the 16S rRNA gene}

Genomic DNA extraction from a single sample at each time point was performed using the Quick-DNA ${ }^{\mathrm{TM}}$ Fecal/Soil Microbe Miniprep Kit (Zymo Research) according to the manufacturer's instructions.

Illumina 16S rRNA gene amplicon libraries were generated and sequenced by BaseClear (Leiden, the Netherlands). In short, barcoded amplicons from the V3-V4 region of 16S rRNA genes were generated using a 2-step PCR. 10-25 ng genomic DNA was used as template for the first PCR with a total volume of $50 \mu \mathrm{l}$ using the 341F (5'-CCTACGGGNGGCWGCAG$\left.3^{\prime}\right)$ and the 785R (5'-GACTACHVGGGTATCTAATCC-3') primers (29) appended with Illumina adaptor sequences. PCR products were purified (QIAquick PCR Purification Kit) and the size of the PCR products were checked on a Fragment analyzer (Advanced Analytical, Ankeny, US) and quantified by fluorometric analysis (Qubit ${ }^{\mathrm{TM}}$ dsDNA HS Assay Kit). Purified PCR products were used for the 2nd PCR in combination with sample-specific barcoded primers (Nextera XT index kit, Illumina). Subsequently, PCR products were purified, checked on a Fragment analyzer and quantified, followed by multiplexing, clustering, and sequencing on an Illumina MiSeq with the paired-end (2x) 300 bp protocol and indexing. The sequencing run was analyzed with the Illumina CASAVA pipeline (v1.8.3) with demultiplexing based on sample-specific barcodes.

The raw sequencing data produced was processed using tools available in QIIME 2 (Version 
2017.11) (https://qiime2.org). Reads were imported and quality filtered and dereplicated with q2-data2 (30). Dada2 was performed with paired-end reads. Truncation parameters were as follows: for forward reads the first 17 base pairs were trimmed off, and truncated at position 280 base pairs; for reverse reads the first 21 base pairs were trimmed off, but these were truncated at position 230 base pairs. The resulting data was used for further analysis. The q2phylogeny plugin (https://github.com/qiime2/q2-phylogeny) was used to generate a tree for phylogenetic diversity analyses. Alpha-diversity (Shannon, Evenness and Faith's PD) and beta-diversity analyses were performed with the q2-diversity plugin (https://github.com/qiime2/q2-diversity). At study I, study II, and study III, in order to include all the samples, we selected 15177, 17638, and 9227 as sampling-depth, respectively. Inverse Simpson was calculated by the package vegan in R. Shannon, Faith's PD, and inverse Simpson were compared among starch sources (or non-starch polysaccharides sources) and time points by a Two-way-ANOVA by the R function aov() in the built-in R package, and multiple pairwise-comparison between the means of groups was performed by Tukey HSD (Tukey Honest Significant Differences, R function: TukeyHSD()) in the built-in R package. The results were visualized by ggpubr $\mathrm{R}$ package. Distance matrices were calculated by unweighted (qualitative) and weighted (quantitative) UniFrac to compare the microbiota of individual units used in the study, where the former only considers their presence or absence, whereas the latter accounts also for abundance of observed organisms (31). In QIIME2 the script "qiime diversity beta-group-significance --i-distance-matrix --m-metadata-column -p-method --p-pairwise --p-permutations --o-visualization" was used for significance testing on distance matrices. The script performs PERMANOVA analyses (32). Factors of starch sources and non-starch polysaccharides were tested with PERMANOVA. Taxonomic analysis was done using the q2-feature-classifier plugin (33). The classifier was trained on Silva_128_release using the $99 \%$ identity level for amplicon sequence variant (ASV), where the sequences had been trimmed to only include 400 bases from the region of the $16 \mathrm{~S}$ that was sequenced in this analysis (the V3-V4 region, bound by the Bakt_341F/ Bakt_785R primer pair). Networks were constructed by Conet (34), analyzed by NetworkAnalyzer (35) and visualized in Cytoscape (36). For constructing co-occurrence networks, the feature (ASV) table was split into three datasets: maize starch, potato starch, and wheat starch. For each of the datasets, only the ASV that appeared in 3 or more in the total of 6 samples were kept for downstream analysis. Five methods (Pearson, Spearman, Mutual Information, Bray Curtis and Kullback-Leibler dissimilarity) were selected for the ensemble inference. MCODE (37), a Cytoscape plugin, was used to uncover modules in the three networks. Linear discriminant analysis effect size [LEfSe] (38) was used to find biomarkers between groups using relative abundances from the feature tables generated in QIIME. The feature tables were filtered as follows: only ASV with a mean of all the samples greater than $10^{-5}$ were kept; taxa not seen more than 3 times in at least $20 \%$ of the samples were removed before LEfSe analysis. Phylogenetic Investigation of Communities by Reconstruction of Unobserved States [PICRUSt] (39) was used to predict the gene families contributing to the metagenomes of the samples identified using 16S rRNA sequencing (KEGG database was used to predict the results). This data was visualized using statistical analysis of taxonomic and functional profiles (STAMP) (40). Raw sequencing data was submitted to the European Nucleotide Archive under the accession number: PRJEB31280.

\subsubsection{SCFA (acetate, propionate and butyrate) analysis}

Samples for determination of concentrations of SCFA were analyzed by Brightlabs (Venlo, The Netherlands). Ion exclusion chromatography (IEC) was applied on an 883 Ion 
Chromatograph (IC; Metrohm, Switzerland), using a Transgenomic IC Sep ICE-ION-300 column (30 cm length, $7.8 \mathrm{~mm}$ diameter and $7 \mu \mathrm{m}$ particles) and a MetroSep RP2 Guard. The mobile phase consists of $1.5 \mathrm{mM}$ aqueous sulphuric acid. A column flow rate of $0.4 \mathrm{ml}$ $\min ^{-1}$ was used. The temperature of the column was $65^{\circ} \mathrm{C}$. The organic acids were detected using suppressed conductivity detecting. Samples were centrifuged at 14,000 rpm (K243R Refrigerated Centrifuge, PrO-Research, UK) for 10 minutes, and the clear supernatant was filtered through a $0.45 \mu \mathrm{m}$ PFTE filter and diluted with mobile phase (for lumen 1:5, for dialysate 1:2). Ten microliters were loaded on the column by an autosampler 730 (Metrohm). Molecules were eluted according to their pKa. Significance of SCFA concentrations among different treatments was analyzed by ANCOVA with the built-in R function aov() (version 3.5.2), and bar charts were made in Microsoft Office. 


\section{RESULTS}

\subsection{The Effect of Different Starch Sources on Pig Microbiota}

\subsubsection{Microbiota diversity}

Alpha-diversity analyses were performed on the samples from each unit of the in vitro model over the 72 hour period and showed that there was no significant difference in Shannon index among the different diets. However, Faith's PD and inverse Simpson indices were significantly changed among the different diets (Figure S2). The Faith's PD index of the microbiota treated with maize starch was significantly higher than that of microbiotas treated with potato or wheat starch. The inverse Simpson index of the microbiota fed with wheat starch was considerably higher than that fed with maize or potato starch (Figure S2). Shannon, Faith's PD, and inverse Simpson indices of the microbiotas treated with the three different starches decreased over time, where the wheat starch fed group fell at the fastest rate, followed by potato starch and maize starch fed groups, respectively (Figure S2). All indices were significantly lower at time point 72 hours, compared to time point 24 hours (Figure S2). Figure 1 demonstrates that pig microbiota communities were clustered according to the substrate that was fed (unweighted: $\mathrm{P}=0.001$, weighted, $\mathrm{P}=0.001$ ) (Figure 1). The dissimilarity in $\beta$-diversity within the same time points of wheat starch fed microbiota assessed with weighted UniFrac was bigger than that with unweighted UniFrac, which demonstrated that ASV abundance affected the dissimilarity of the wheat starch fed microbiota. A PERMANOVA analysis of the Bray-Curtis distances showed a similar results, where Bray-Curtis metrices were significantly different among the three starch sources (data not shown). After feeding maize, potato, and wheat starch, the original inoculum sample separated with samples from maize, potato, and wheat starch.
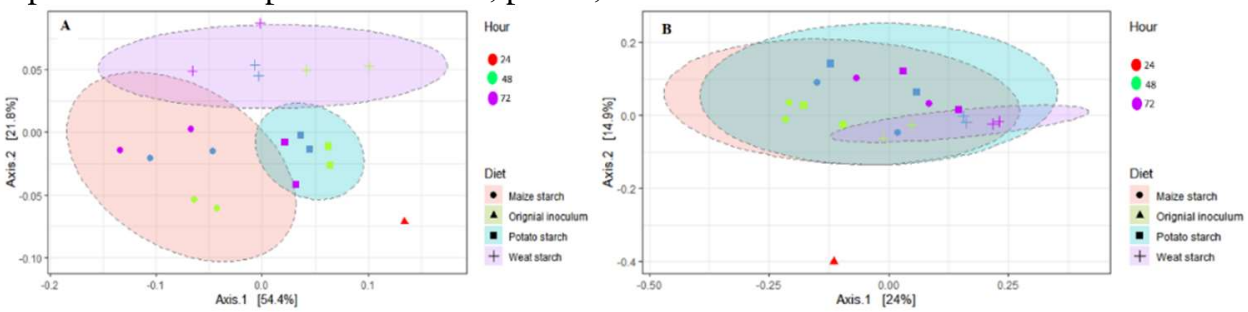

Figure 1: Principal coordinate analysis (PCoA) plots generated based on the calculated distances in a weighted (A) and unweighted (B) UniFrac matrix. Samples were grouped by color (time-points) and shape (in terms of diet group) they belonged to. The ellipses were drawn at the 0.95 confidence level. Diet sources and original were significant different from each other, for weighted uniFrac: Original inoculum to Maize starch $(\mathrm{P}=0.041)$, to Potato starch $(\mathrm{P}=0.031)$, to Wheat starch $(\mathrm{P}=0.033)$; Maize starch to Potato starch $(\mathrm{P}=0.002)$, to Wheat starch $(\mathrm{P}=0.002)$; for unweighted uniFrac: Original inoculum to Maize starch $(\mathrm{P}=0.046)$, to Potato starch $(\mathrm{P}=0.041)$, to Wheat starch $(\mathrm{P}=0.048)$; Maize starch to Potato starch $(\mathrm{P}=0.022)$, to Wheat starch $(\mathrm{P}=0.006)$.

\subsubsection{Taxonomic affiliations}

The dominant bacterial phyla were Firmicutes $(48 \%, 46 \%, 48 \%$, and $50 \%$ in maize starch, potato starch, wheat starch and original inoculum experiments, respectively), Bacteroidetes (17\%, 27\%, 24\%, and 34\% respectively), and Actinobacteria (33\%, 17\%, 12\%, and 11\% respectively) (Figure 2A, Figure S3). Figure 2B and Figure S3 shows the 14 most abundant families of the original inoculum and the three experimental groups. The relative abundance of the Prevotellaceae family in the original inoculum was much higher than those in maize, 
potato, and wheat starch groups. At both phylum and family level, the microbial relative abundances between duplicates were quite similar and the bacterial abundances among the three different time points were also stable (Figure S3).
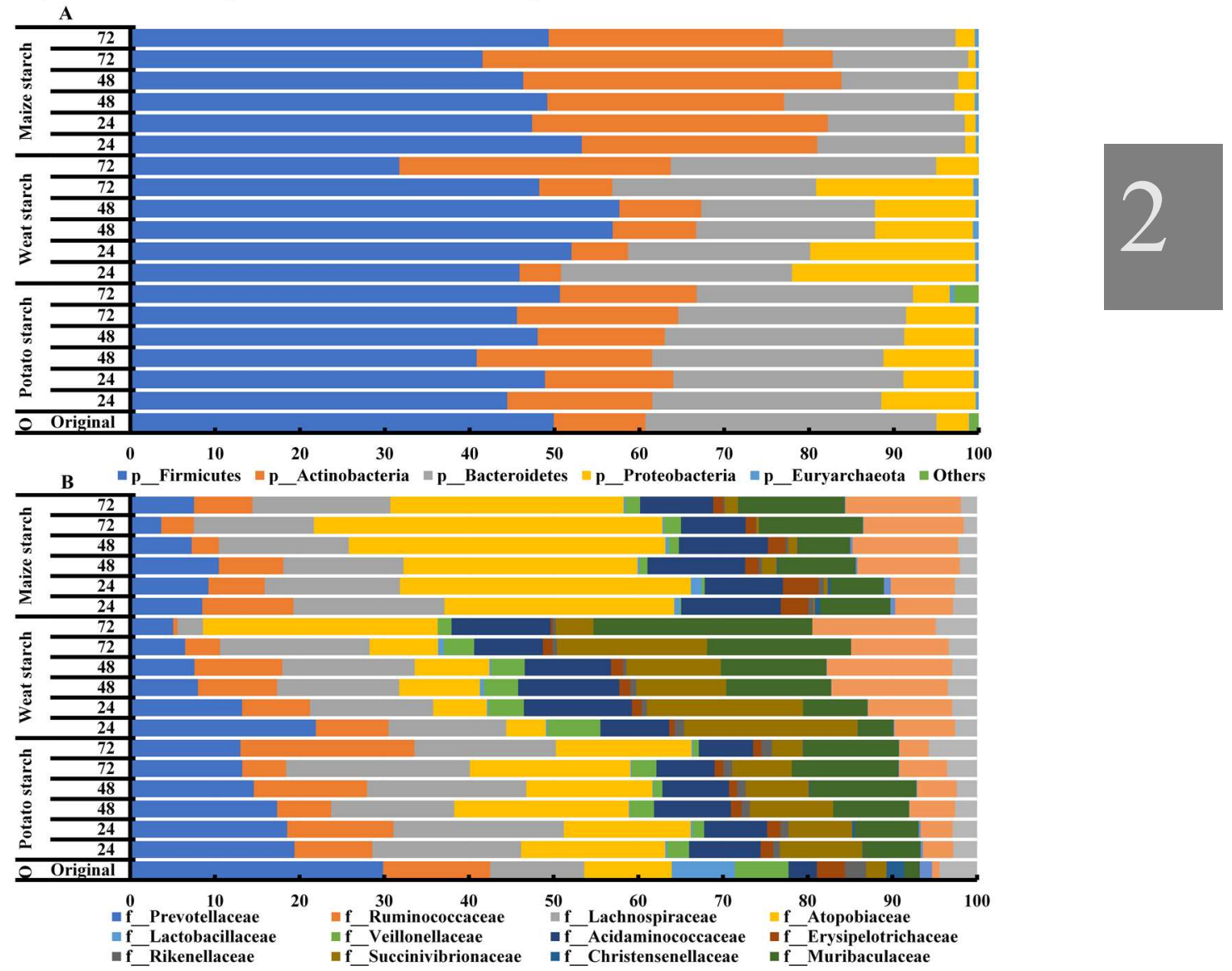

Figure 2: Relative abundances of microbial phyla (A) and families (B) in the original inoculum, and microbiome fed with different starches at different time points $(24 \mathrm{~h}, 48 \mathrm{~h}$, and $72 \mathrm{~h})$.

Within the phylum Firmicutes, the families Lactobacillaceae, Streptococcaceae, Christensenllaceae, Clostridiales, Family XIII, and Erysipelotrichaceae were more abundant in maize starch treated microbiota compared to potato and wheat starch, while Acidaminococcaceae and Veillonellaceae showed higher abundance upon wheat starch addition compared to the other two starches (Figure 3). Among Bacteroidetes, Prevotellaceae was more abundant upon potato starch feeding compared to the other two starch substrates. Among Actinobacteria, Coriobacteriaceae was more abundant for maize starch, while Bifidobacteriaceae was more abundant for wheat starch (Figure 3). Within Proteobacteria, Alcaligenaceae, Succinivibrionaceae, and Enterobacteriaceae were more abundant for wheat compared to maize and potato starch (Figure 3). At the genus level (Figure S4), the relative abundance of Coriobacteria, Olsenella, Holdemanella, Acidaminocuccus, Mogibacterium, 
Solobacterium, Lactobacillus, Coprococcus3, Streptococcus, Dorea, and Catenibacterium were significantly higher for maize starch, while the relative abundance of Prevotella9, Acetitomaculum, Ruminococcus2, Coprococcus1, Syntrophococcus, Sharpea, and Mitsuokella were significantly higher for potato starch group. The relative abundance of Succinivibrio, Negativicutes, Megasphaera, Bifidobacteriales, Actinobacteria, Citrobacter, and Sutterella were significantly higher for wheat starch.
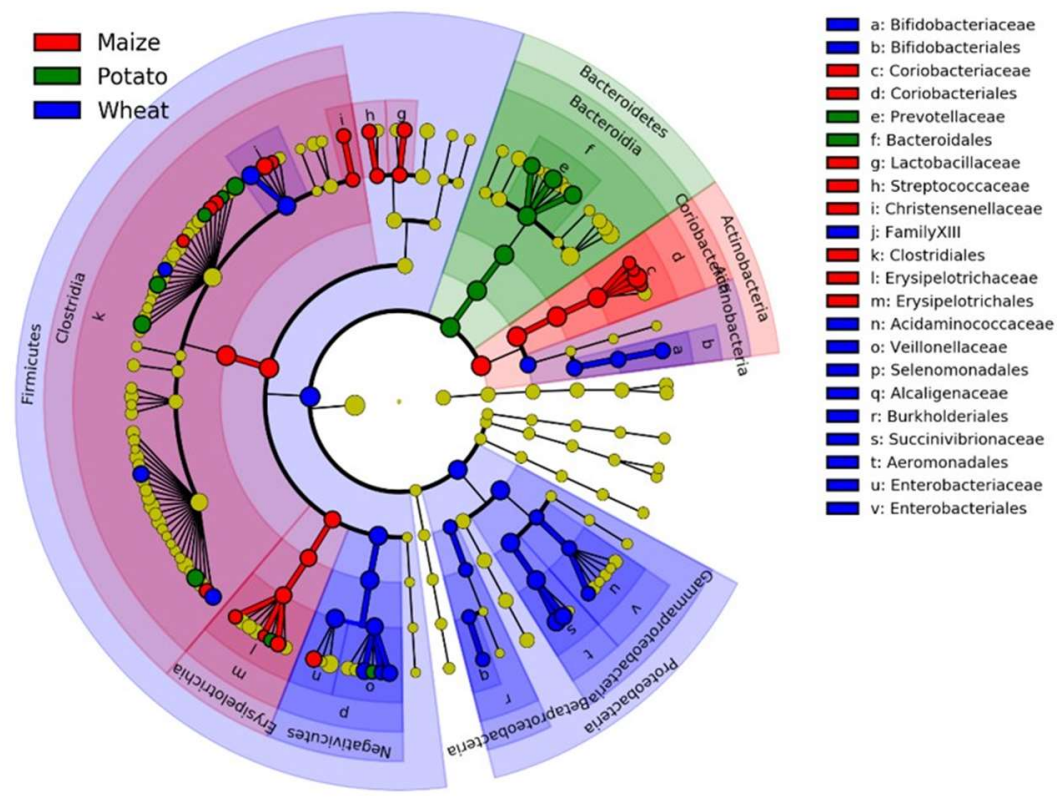

Figure 3. LEfSe results on starch-fed pig microbiota showing taxonomic representation of statistically and biologically consistent differences among maize, potato, and wheat starch treated microbiota. Differences are represented by the color of the substrates (red, green, and blue indicating Maize, Potato, and Wheat starch, respectively).

\subsubsection{Ecological network}

As shown in Figure S5 and Table 1, the topological structures were different among maize, potato, and wheat starch. There were 325 nodes connected by 3349 edges, 239 nodes connected by 2874 edges, and 264 nodes connected by 3194 edges in maize, potato, and wheat starch-based networks, respectively. This indicates that maize starch-based network was larger. The $\mathrm{R}^{2}$ fitting of the three networks was 0.709 (maize starch), 0.543 (potato starch), and 0.536 (wheat starch), respectively. This suggests that the scale-free property of the maize starch-based network was better than those of the networks for potato and wheat starch.

To detect dense modules and to explore the biological patterns in the three starch networks, the tool MCODE (37), a plugin for Cytoscape, was applied to analyze the networks. We detected 14 modules, 8 modules, and 15 modules present in maize, potato, and wheat starch networks, respectively. Figure 4 shows the top three highly-connected modules present in 
each of the three starch networks. In the largest module (module 1), maize starch contained 30 nodes connected by 624 edges. Among them, 20 nodes belonged to the phylum Firmicutes, and the rest nodes belonged to Bacteroidetes (6 nodes), Actinobacteria ( 3 nodes), and Protobacteria (1 node). Module 1 of Potato starch contained 32 nodes and 565 edges, among which 16 nodes and 11 nodes belonging to Firmicutes and Bacteroidetes, respectively. The other 5 nodes were shared by the phyla Actinobacteria, Proteobacteria, and Euryarchaeota. With respect to wheat starch, the sub-network (module 1), contained 35 nodes and 803 edges, among which both Bacteroidetes and Firmicutes had 14 nodes, and Proteobacteria and Actinobacteria had 5 nodes and 2 nodes, respectively.
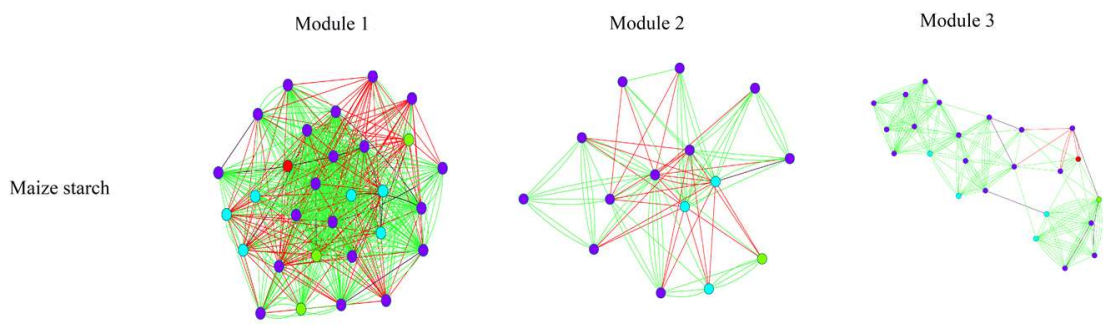

Potato starch
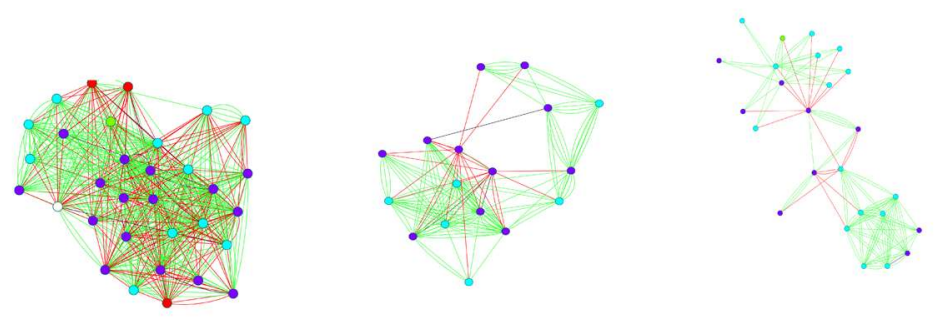

Wheat starch
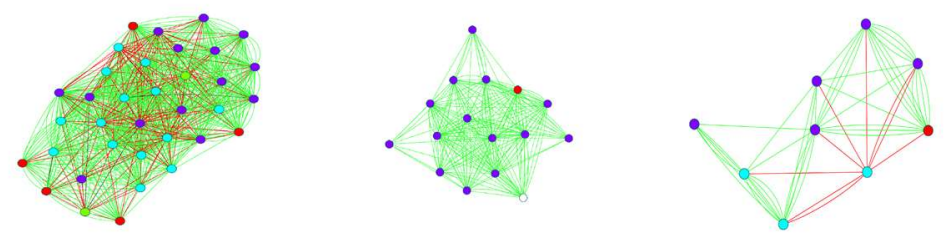

Actinobactera $=$ Bacteroidetes

Firmicutes

- Proteobacteria

Figure 4: Top three highly-connected clusters in networks of porcine gut microbiota fed with maize, potato, and wheat starch. Node colors represent the different phyla. Green edges represent positive interactions, while red edges indicate negative interactions, and black ones are unknown.

To reveal the hub nodes of each network, the degree (number of edges connected to a node), positive degree (number of positive edges connected to a node), and negative degrees (number of negative edges connected to a node) were assigned to each node by Conet (Table S1) (34). The top connected nodes of maize, potato, and wheat starch were Tuicibacter, Subdoligranulum, and Prevotella, respectively, which were also their top positive hubs. The 
top negative hubs belong to Megasphaera, Ruminococcaceae UCG-014, and Prevotellaceae, respectively.

\subsubsection{Predictive functional profile}

Given the structural changes within the bacterial community among maize, potato, and wheat starch treatment, we subsequently tested whether different starches caused functional profile changes within each microbiota too, and we compared this to an original pig fecal sample to reveal the functional similarity between different starch groups. PICRUSt (39) was applied to the 16S rRNA gene data to predict functional content of the metagenome. Twenty-eight active features were found to be significantly different among the three starch groups (Table S2). More specifically, in the top five most abundant differential features, the relative abundance of Membrane Transport for maize starch was significantly higher than the other two groups (maize starch : wheat starch, $\mathrm{P}<0.001$; maize starch : potato starch, $\mathrm{P}<0.01$ ). Moreover, for potato starch this was also significantly higher than for wheat (potato starch : wheat starch, $\mathrm{P}<0.05)$. The relative abundance of Replication and Repair for maize starch was significantly lower (wheat starch : maize starch $\mathrm{P}<0.001$; potato starch : maize starch, $\mathrm{P}<0.001$ ) (Table S2). The relative abundance of Carbohydrate Metabolism for maize starch was significantly higher than the other two starches (maize starch : wheat starch, $\mathrm{P}<0.01$; potato starch : wheat starch, $\mathrm{P}<0.01$; maize starch: potato starch, $\mathrm{P}<0.01$ ), and that of wheat starch lower than that of potato starch. The relative abundance of Amino Acid Metabolism for wheat starch was significantly higher (wheat starch : potato starch, $\mathrm{P}<0.001$; wheat starch : maize starch, $\mathrm{P}<0.001)$. The relative abundance of Translation for potato starch was significantly higher than that for wheat starch $(\mathrm{P}<0.05)$ (Table $\mathrm{S} 2)$.

The relative abundance of microbial functional profiles of the three starch groups and the original pig feces were used to create a PCA plot (Figure 5A) and heatmap (Figure 5B). Hierarchical clustering showed that samples from the same starch tended to cluster together, and the original pig microbiota clustered with the maize starch treated microbiota. The same result was observed in the PCA plot: samples from different starch treatments clearly separated, and the sample from the pig feces clustered with samples from maize starch (Figure 5A).

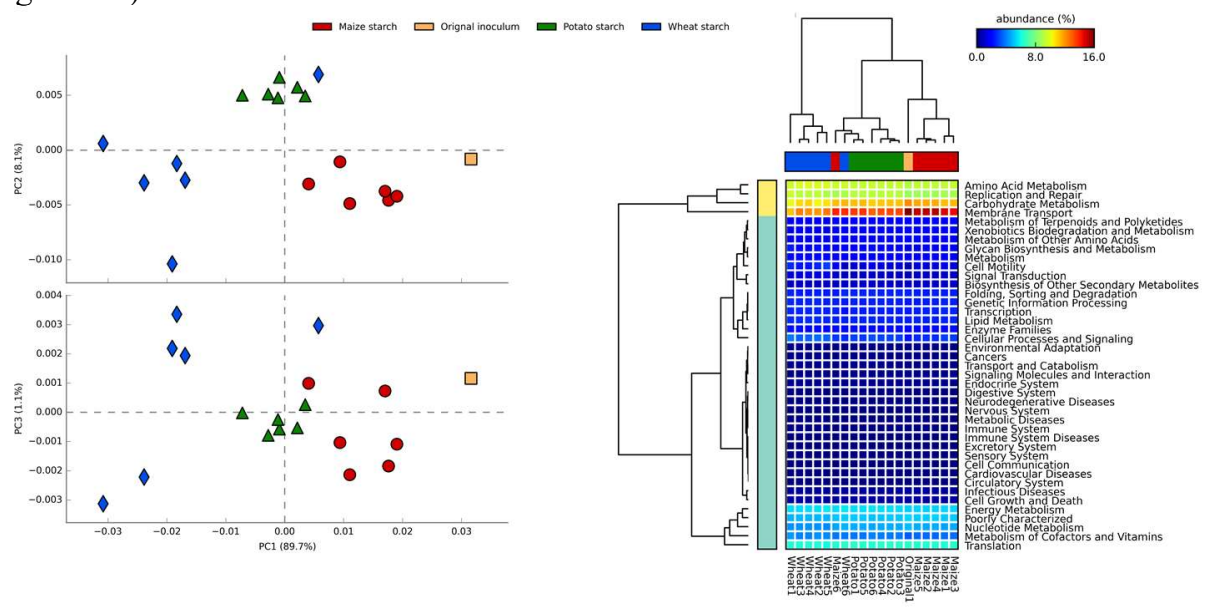

Figure 5. PCA (A) and heatmap (B) plot of microbial functional profile of maize (red), potato (green), and wheat starch (blue) treatments and the original pig fecal sample (orange). 


\subsubsection{Microbiota activity}

Figure 6 shows the cumulative production of beneficial microbial metabolites SCFA (acetate, propionate and butyrate) after 72 hours of maize, potato, and wheat starch treatment. The cumulative amounts of acetic $(\mathrm{P}=0.006)$, propionic $(\mathrm{P}=0.008)$, and butyric acid $(\mathrm{P}=0.034)$ produced upon maize starch feeding were significantly higher than for potato starch and wheat starch. This corroborated the predicted functional data that the relative abundance of Carbohydrate Metabolism for maize starch was significantly higher than that of the other two groups. SCFA production did not differ between wheat and potato starch.

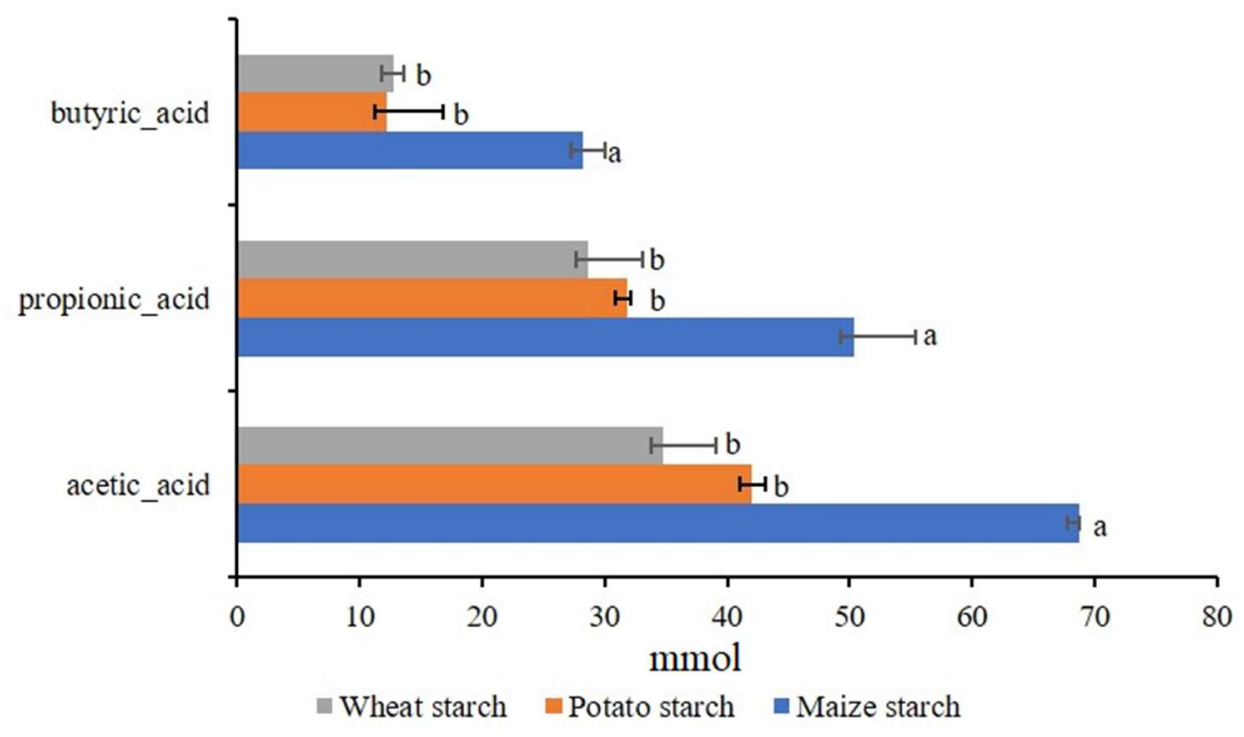

Figure 6. Effect of different starches on the SCFA after 72 hours fermentation

\subsection{Study II: The Effect of Different Non-Starch Polysaccharides on Pig Microbiota}

Study I proved that predictive functional profile composition for maize starch treatment was most similar to that of the original pig inoculum. Therefore, maize starch was chosen as starch source in the SIEM recipe in study II. SIEM is optimized for humans. But, as the diet of human beings is different from pigs, the composition of other polysaccharides (besides starch source) should also be different. Moreover, pigs may obtain more fibers from their diet. Consequently, two more polysaccharides, which are present in maize or soybean meal, were added into the recipe of SIEM (arabinoxylans, AX; or arabinoxylans plus xyloglucan, AX$\mathrm{XG}$ ) to investigate their effect on pig microbiota functionality. The microbiome functional profile data of maize group of study I and the original pig feces was used to compare their differences among AX, and AX-XG group.

\subsubsection{Microbiota diversity}

There was no significant differences in Shannon index and Faith's PD between AX and AXXG feeding (Figure S6), but inverse Simpson of microbiota fed with AX-XG was significantly higher than that fed with AX. Shannon index and inverse Simpson of AX-XG were slightly higher than those of maize starch, but they were not significant (data not shown). Additionally, Shannon index, Faith's PD, and inverse Simpson of time point 24 hours were significantly higher than those of time point 48 and 72 hours. 
The added polysaccharides significantly affected beta-diversity (Figure S7). The weighted and unweighted UniFrac were used to calculate the phylogenetic distance matrix, and a PERMANOVA test was performed in each case to determine if the separation of sample groups was significant. The PERMANOVA test showed microbial profiles of AX treatment separated significantly from that of $\mathrm{AX}-\mathrm{XG}$ treatment (weighted: $\mathrm{P}=0.003$, unweighted: $\mathrm{P}$ $=0.034)$.

Beta-diversity of maize starch from study I was introduced to compare with AX and AX-XG. There were significant differences between maize starch and AX-XG in beta-diversity (weighted: $\mathrm{P}=0.003$, unweighted: $\mathrm{P}=0.024)$. The difference in unweighted UniFrac between maize starch and AX was not significant $(\mathrm{P}=0.141)$, while it was significant for weighted UniFrac $(\mathrm{P}=0.003)$. Figure $\mathrm{S} 7$ shows that difference among samples in $\mathrm{AX}-\mathrm{XG}$ was smaller than those in $\mathrm{AX}$, which means $\mathrm{AX}-\mathrm{XG}$ had less individual difference and highly reproducibility between replicates.

\subsubsection{Taxonomic affiliations}

Stacked relative abundance bar plots were produced for all samples at phylum and family level (Figure 7 and Figure S8). Compared to the microbiota fed with maize starch (containing 17\% Bacteroidetes, 33\% Actinobacteria), the relative abundance of Bacteroidetes was increased in the microbiota fed with AX (28\%), and AX-XG (30\%), and the relative abundance of Actinobacteria was deceased for AX (22\%), and AX-XG (19\%), which made the microbial composition closer to the original inoculum (34\% Bacteroidetes, $10 \%$ Actinobacteria). At phylum level, the microbial composition of AX-XG was more similar to the original inoculum than that of AX. Similar results are also shown at the family level. After feeding with $\mathrm{AX}$ and $\mathrm{AX}-\mathrm{XG}$, relative abundances of the families Prevotellaceae, and Succinivibrionaceae were increased and that of family Atopobiaceae was decreased, compared to the microbiota fed with maize starch, which made the microbiota composition more similar to the original inoculum. But all treatments failed to maintain similar levels of the families Lactobacillaceae and Christensenellaceae, which had higher relative abundance in the original inoculum (Figure 7). The microbial abundances (at phylum and family level) between duplicates were quite similar (Figure 7), and were stable among different time points, except for family Ruminococcaceae. During the first 24 hours of incubation, the relative abundance of Ruminococcaceae was quite similar to the original inoculum, but it dropped down at 48 hours and 72 hours. 


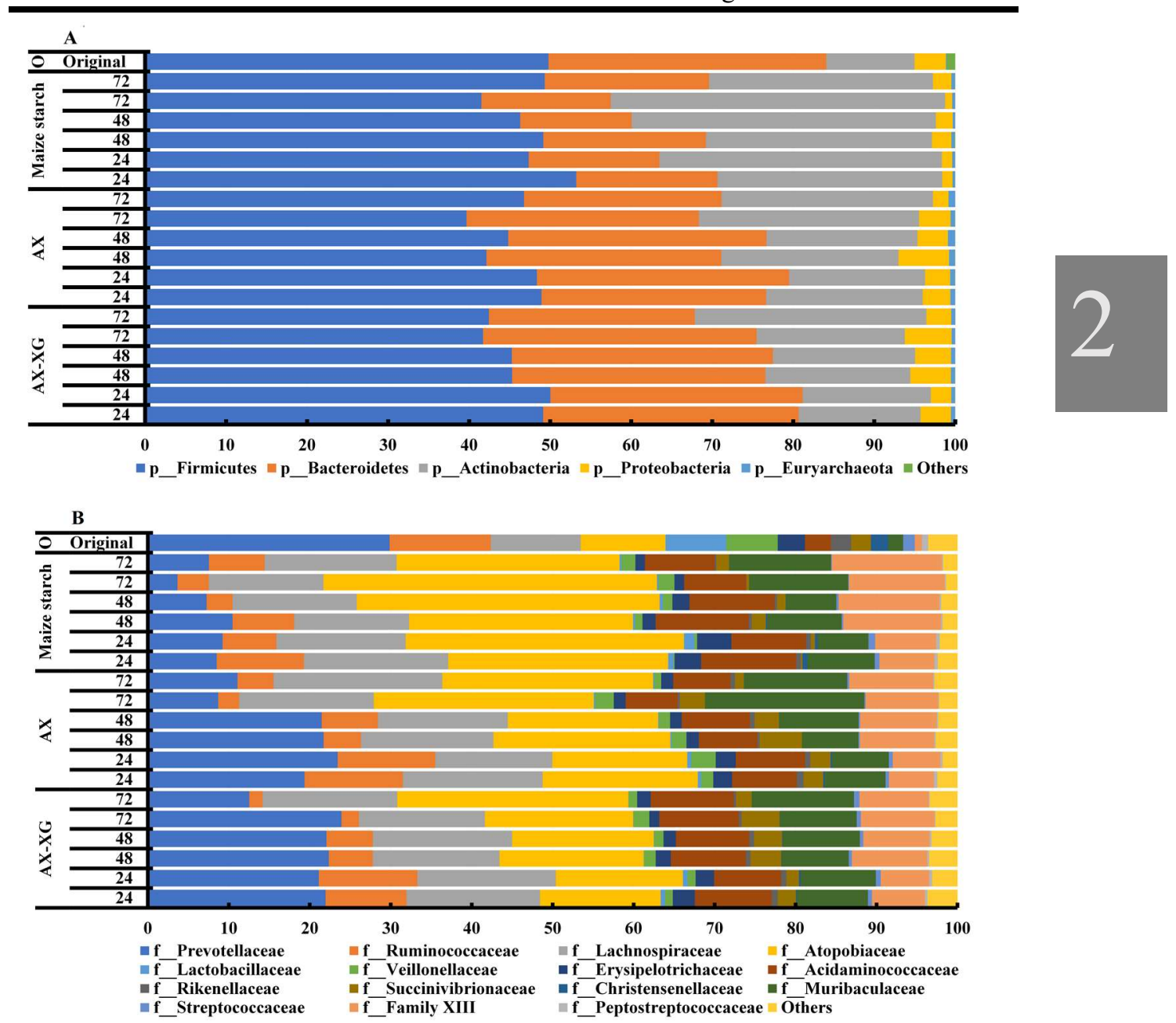

Figure 7. Relative abundances of microbial phyla and families in the original inoculum, and microbiome fed with different substrates at different time points ( $24 \mathrm{~h}, 48 \mathrm{~h}$, and $72 \mathrm{~h}$ ).

At the genus level, the core genera in the original inoculum, and the microbiotas fed with different polysaccharides are shown in a PCA plot (Figure 8A), showing that the genus composition of the original inoculum was closer to $\mathrm{AX}-\mathrm{XG}$, compared to maize starch and AX. And samples in maize starch and AX also had larger individual differences than samples in $A X-X G$. 


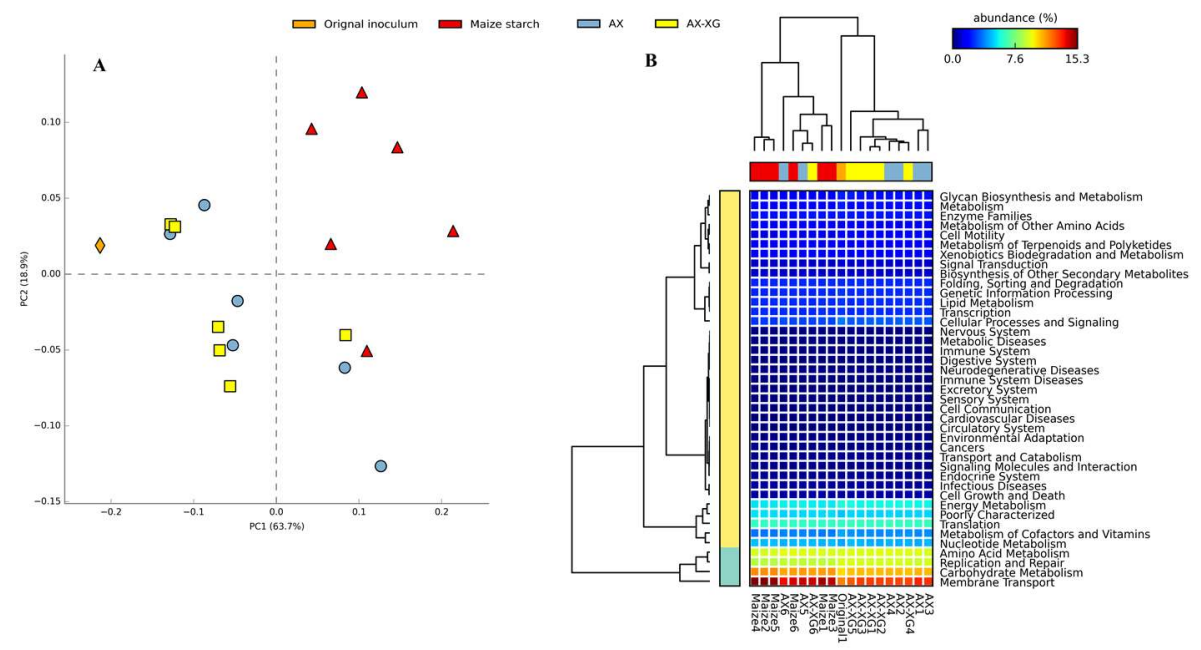

Figure 8. PCA (A) plot of microbial relative abundance at genus level of maize (red), AX (blue), and AX-XG (yellow) treatments and the original pig fecal sample (orange); Heatmap plot (B) of microbial functional profile of maize (red), AX (blue), and AX-XG (yellow) treatments and the original pig fecal sample (orange).

Most of the significant taxa affected by the treatment were observed in the AX-XG group (Figure S9). At the family level, four families were more abundant upon AX-XG treatment than after AX feeding: Methanobacteriaceae, Enterococcaceae, Streptococcacceae, and Acidminococcaceae. In contrast, only one family (Bacillaccae) was more abundant for AX than for AX-XG. At the genus level, Prevotella7, Lactococcus, Succiniclasticum, Enterobacter, and Escherichia_Shigella, were more abundant for AX-XG, while Bacillus had a significantly lower abundance compared to AX. There were no significantly difference at genus level between AX-XG and original inoculum, excepting for [Ruminococcus] gauvreauii group and Dialister (Table S7).

\subsubsection{Predictive functional profile}

Metagenome function was predicted using PICRUSt to investigate whether the diets would change the microbiome functional profile. Despite some differences in beta-diversity, only one significant feature was found, Immune system, of which the relative abundance was significantly higher $(\mathrm{P}=0.034)$ for $\mathrm{AX}$. Subsequently, the predicted microbiome functional profiles of the maize starch treatment from Study I and the original pig fecal sample were compared to that of AX and AX-XG group (Figure 8B). The heatmap plot (Figure 8B) shows that the relative abundances of the predictive functional metagenome of the original inoculum were similar to $\mathrm{AX}$ and $\mathrm{AX}-\mathrm{XG}$, but it clustered more with $\mathrm{AX}-\mathrm{XG}$.

\subsubsection{Microbiota activity}

Table S3 shows that there were no significant differences between AX and AX-XG with respect to the cumulative production of the beneficial metabolites propionate and butyrate after 72 hours fermentation. Acetate production was comparable for the two substrates, yet the amount of Acetate for AX-XG was significantly higher than that for AX $(\mathrm{P}<0.001)$.

\subsubsection{Reproducibility of microbiota fed with AX-XG}

As described above, AX-XG was the optimized medium for SLIM, thus its microbial reproducibility was assessed by its replicates of alpha diversity, beta diversity, microbial 
abundance, and microbial activities. The coefficients of variation (C.V.) of Shannon index and Faith_PD at time points 24, 48, and $72 \mathrm{~h}$ were all lower than $6 \%$ (Table S4). Most of the C.V. of microbial abundance (at phylum and family level) were lower than $15 \%$, only 5 taxa, which represented $9.8 \%$ of total taxa, were higher than $15 \%$ (Table S5). And most of them were lower than $10 \%$. C.V. of acetic acid, propionic acid, and butyric acid were all lower than $14 \%$, and most of them were lower than $5 \%$ (Table S6).

\section{Discussion}

The TNO computer-controlled dynamic in vitro model of the colon (nick-named TIM-2) was developed by TNO approximately 20 years ago (18), and has been used extensively to study the effect of all kinds of dietary substrates on the human gut microbiota. For instance, the effect of probiotics and antibiotics on the intestinal homeostasis (41), the effect of numerous carbohydrates $(42,43)$, or fermentation of polyphenols from citrus, tea, or soy flavonoids into phenolic compounds with a more simple structure (44). The model is highly reproducible, as it is completely computer-controlled. Here, in order to investigate the pig gut microbiota in vitro, we modified the in vitro system to the swine colon (SLIM). As TIM-2 is originally designed for human beings, the colon parameters, such as temperature $\left(37^{\circ} \mathrm{C}\right), \mathrm{pH}(5.8)$, and Standard Ileal Efflux Medium (SIEM), are adapted to the physiology of man. For SLIM, therefore temperature $\left(39{ }^{\circ} \mathrm{C}\right), \mathrm{pH}(5.9)$ and medium composition were modified to correspond to swine physiology $(45,46)$. Next generation sequencing was used to evaluate the effect of different starch sources and polysaccharides added to the SIEM medium on the microbiota of finishing pigs in the in vitro model during 72-hour fermentation experiments to investigate the optimal medium composition for pig gut microbiota. This study showed that starches from three different sources had significantly different effects on microbiota composition of finishing pigs and their predicted functional profiles. The microbial composition and predicted functional profile of original inoculum was similar to those of the microbiota fed with AX-XG, compared with the microbiota fed with maize starch and AX. Moreover, pig microbiota composition and activity fed with SIEMP (comprising of maize starch, AX, XG, and the rest of the compounds in normal (human) SIEM) were highly reproducible.

\subsection{The Effect of Different Starch Sources on Pig Microbiota}

Normally carbohydrates fermented by bacterial community in the colon belong to non-starch polysaccharides, but research showed that in humans 8-40 g starch per day may reach the colon $(47,48)$, which is termed resistant starch (49). In pigs, there is already substantial fermentation in the distal small intestine, and thus the amount of resistant starch that may reach the cecum/colon in pigs may differ, but we were unable to find any data on this. Resistant starch can be classified into four types, which are RS1 (physically inaccessible), RS2 (resistant granules), RS3 (retrograded starch), and RS4 (chemically modified). Feed processing, like heat, moisture, and pelleting, will affect the starch availability and formation of different types of RS (50). This RS reaching the swine colon will affect the microbiota composition and host health. In this in vitro study, SIEM (containing potato starch) was heated and mixed with other feed compounds during the preparation, and it contains RS. But the SIEM medium was developed to mimic a human diet. Thus, we aimed to investigate how the pig microbiota changes after feeding different sources of (resistant) starch.

Starch sources impacted the microbiota composition. Different starch sources had a considerable effect on both alpha-diversity (inverse Simpson, and Faith's PD), and betadiversity (weighted and unweighted UniFrac, and Bray-Curtis) (Figure S2, Figure 1), indicating that some microbial species (composition and/or abundance) among the three 
starch treatments were significantly different. Previous studies showed that certain starches can impact gut microbiota composition $(4,51)$. (4) demonstrated that microbiota fed with starches with the same RS type clustered together, while starches with different RS types significantly separated (4). (52) showed retrograded potato starch (RS3), but not native potato starch (RS2), promotes fecal and cecal Lactobacillus counts in rats. Another study showed that a chemically modified RS (RS4) increased Bifidobacterium adolescentis and Parabacteroides distasonis, while a RS2 led to significant increases in the proportions of Ruminococcus bromii and Eubacterium rectale when compared to RS4 (53). In the current study, among the three starch treatments, 11 genera, 7 genera and 7 genera were more abundant upon maize, potato, and wheat starch treatment, respectively (Figure 3, Figure S4). The inherent properties of starches, such as crystallinity, granular structure, amylose:amylopectin ratio, and retrogradation of amylose, will influence the formation of resistant starch (54). Therefore, the proportion and amount of RS types of starch sources may lead to alteration of the microbiota composition after feeding these starches.

The ensemble method was used based on multiple similarity measures in combination with generalized boosted linear models on microbial abundance (55). This investigates whether the shift of microbial abundance will change the bacterial co-occurrence relationships. Our results showed that maize starch-based network had much better scale-free properties, as evaluated by the value of degree distribution fitting $\mathrm{R}^{2}$ (Table 1). Scale-free networks display a very strong robustness against random failures (56). Thus microbiota fed with maize starch may be more stable or resilient, compared to those of potato and wheat starch.

Table 1 Topological properties of networks of porcine gut microbiota fed with different sources of starch.

\begin{tabular}{lccc}
\hline Network property & Maize starch & Potato starch & Wheat starch \\
\hline Number of nodes & 325 & 239 & 264 \\
Number of edges & 3349 & 2874 & 3194 \\
Clustering coefficient & 0.361 & 0.389 & 0.476 \\
Network diameter & 10 & 8 & 8 \\
Characteristic path length & 3.369 & 3.188 & 3.127 \\
Network density & 0.041 & 0.069 & 0.058 \\
Network heterogeneity & 1.088 & 1.027 & 1.005 \\
Multi-edge node pairs & 778 & 628 & 756 \\
Degree distribution fitting slope & -0.807 & -0.617 & -0.626 \\
Degree distribution fitting R & & 0.543 & 0.536 \\
\hline
\end{tabular}

Networks of microbiota fed with different sources of starch had different keystone species (Figure 4, Table S1). The top ten highly-connected nodes in the maize starch-based network were all different from those of potato and wheat starch-based network. Moreover, Figure 4 shows that the top three highly-connected clusters of the maize starch had more hub nodes from the phylum Firmicutes, while potato starch had more hub nodes from the phylum Bacteroidetes. Wheat starch contained more hubs from the phylum Proteobacteria, compared to maize and potato starch. A high ratio of Firmicutes:Bacteriodetes are characterized as a trait of obesity, which goes hand-in-hand with observations of high levels of SCFA in the gut (57-59). In our study, hub nodes of the maize starch-based network showed that it was dominated by Firmicutes ( Figure 4). Likely as a consequence of this, SCFA production of maize starch fed microbiota was significantly higher than that of potato and wheat starch ones (Figure7), and the corresponding (predicted) microbial function was also changed. Metagenome prediction revealed that gene functions associated with Carbohydrate Metabolism were more abundant for maize starch than for potato (the second most abundance) 
and wheat starch (the lowest abundance) (Table S2). Resistant starch is believed to play a key role in SCFA production in the intestine (60). It shifts the SCFA-producing bacteria, resulting in different production of short-chain fatty acids. The relative abundances of Coriobacteria, Holdemanella, Acidaminococcus, Lactobacillus, and Coprococcus3, which are SCFA-producing bacteria (61-63), were significantly higher for maize starch. The SCFA production data corroborated the functional prediction results of Carbohydrate Metabolism. The cumulative amount of acetic $(\mathrm{P}=0.006)$, propionic $(\mathrm{P}=0.008)$, and butyric acid $(\mathrm{P}=$ 0.034 ) produced upon maize starch feeding were all significantly higher than for potato starch and wheat starch (Figure 6). These findings suggest that maize starch was more fermentable than potato and wheat starch. These findings, together with the considerable higher abundant carbohydrates metabolism linked to maize starch, suggest a better overall digestive capacity (digestion in the upper GI tract plus fermentation in the colon) of animals fed with maize basal diets. Therefore, a maize basal diet may improve energy absorption in the large intestine in growing pigs. However, further in vivo intervention studies need to be performed to validate these hypotheses, for starters with respect to the kinetics of fermentation and SCFA production of the different starches.

In order to investigate which functional composition driven by the different starch sources was more similar to the original pig microbiota, a pig fecal sample was included. Both the PCA and the heatmap of the functional predictive profile showed that the original pig fecal sample clustered with maize starch treatment, which indicated that the functional profile of these samples was more similar to the original pig inoculum (Figure 5).

As discussed above, microbiota fed with maize starch may be more stable or resilient, compared to those of potato and wheat starch, which can more closely simulate the microbial ecology in vivo. And the predictive functional metagenome of the microbiota fed with maize starch was more similar to the original inoculum. Thus, maize starch was used as starch source in the recipe of standard ileal effluent medium for pigs (SIEMP). But the microbial composition between microbiota fed with maize starch and the original inoculum still was very different. Figure 1 shows that the original inoculum separated from the microbiota fed with one of the three starch sources, which indicated the different microbial composition between the original inoculum and starch-fed microbiotas. This is reasonable, as pig diets have different carbohydrate sources than human beings. The dominant phyla in the maize starch-fed microbiota were Firmicutes and Actinobacteria, while they were Firmicutes and Bacteroidetes in the original inoculum (Figure 2). Previous reports $(1,7,8)$ have also shown that the most abundant phyla were Firmicutes and Bacteroidetes in pig gut microbiota. Therefore, the study II was performed, in which an attempt was made to adjust the microbial composition in the in vitro model to become more similar to the original inoculum.

\subsection{The Effect of Non-Starch Polysaccharides on Pig Microbiota}

Supplementation of non-starch polysaccharides to the SIEMP on top of maize-starch led to increases of the relative abundance of the phyla Bacteroidetes and Proteobacteria, and the decrease of the relative abundance of phylum Actinobacteria (Figure 7A) eventually leading to the competitive exclusion of Actinobacteria. This is supported by the results reported by (12) showing that AX-fed pigs had a higher abundance of species from the Prevotella cluster from the phylum Bacteroidetes. (6) found that Bacteroides (phylum Bacteroidetes) played a major role in the degradation of AX in children's fecal microbiotas. (64) reported that AX induced a specific increase in Bacteroides-Prevotella spp. and Roseburia spp. At a lower phylogenetic level, similar results were also found in our study. At the family level, the relative abundances of Prevotellaceae in the microbiota fed with AX and AX-XG were more 
than twice times higher compared with that in the microbiota fed with maize starch (Figure 7B). All the three studies mentioned showed that AX can induce increases in Prevotella from the family Prevotellaceae $(6,12,64)$. There are numerous other studies showing that Prevotella is the major genus involved in AX degradation (3, 65-69). AX supplementation also induced the abundance of the family Muribaculaceae from the Bacteroidetes phylum (Figure 7B), which lead to an abundance similar to that of original inoculum. Until now, there is no research about utilization of AX through Muribaculaceae in pigs, but it is known to be a dominant bacterial group in the mouse gut (70). By using 30 population genomes extracted from fecal samples of four different animal hosts (human, mouse, koala, and guinea pig), (71) found that the most abundant glycosidase hydrolases in Muribaculaceae are dominated by xylosidase and arabinosidase enzymes capable of degrading xylan and arabinan, respectively. Thus, we suspect that Muribaculaceae has the capability for degrading AX in the pig gut. Compared to only supplementing AX to maize starch based SIEMP, microbiota fed AX-XG on top of maize-starch had a higher relative abundance of Bacteroidetes and Firmicutes, and a lower relative abundance of Actinobacteria, which made the microbial composition more similar to the original inoculum (Figure 8A). This indicated that the XG supplementation can further increase a relative abundance of bacteria in the phyla Bacteroidetes and Firmicutes, at the expense of the phylum Actinobacteria. (72) confirmed Bacteroidetes can catabolize $\mathrm{XG}$ by a discrete genetic locus, which corroborates that XG supplementation induced the growth of Bacteroidetes in our study. (9) reported that a substantial increase in the $B$. uniformis population occurred after $\mathrm{XG}$ addition to an in vitro fecal fermentation. At a lower phylogenetic level in our study, XG mainly increased the amount of Prevotellaceae, and led to a slight decrease in the number of Atopobiaceae, Lachospiraceae, and Muribaculaceae. We did not find any researches about Prevotellaceae catabolizing XG in pigs. But (73) reported that Prevotellaceae from the rumen microbiota can express xyloglucanses, which are XGdegrading enzymes.

Previous results showed that $\mathrm{XG}$ generates big microbial community shifts during in vitro fecal fermentation (9). Similar results were found in our study; LEfSe results showed that five genera (Prevotella7, Lactococcus, Succiniclasticum, Enterobacter, and Escherichia_Shigella) were found more abundantly upon AX-XG feeding compared to AX alone, while only one genus (Bacillus) was less abundant compared to AX (Figure S9). Moreover, Figure 8 also shows that microbiota shifts occurred during XG fermentation, compared with AX fermentation. It has been hypothesized that isolated $\mathrm{XG}$, an uncommon carbohydrate structure for microbiota fermentation, has the potential to generate larger shifts in microbiota composition than commonly consumed fibers [AX, mainly in wheat bran] (9). Wheat bran, a by-product from milling industry, contains a large proportion of AX and is indeed widely used in pig industry. However, $\mathrm{XG}$, although existing in almost all land plants, is usually strongly embed in the plant's cellulosic cell wall matrix (74) that may cause it to be difficult to be accessed for bacterial fermentation. In our study, pure (isolated) XG was supplemented to the SIEMP and this was fully accessible to microbiota. This uncommon free form of XG might therefore drive to a different fermentation profile than that of AX-only fed pig microbiota. Interestingly, the facultative anaerobic genus Bacillus was found in microbiota fed with AX (average relative abundance, 0.13\%). (75) reported that Bacillus subtilis was found at all intestinal sites and also in mouse feces, which is also supported by other studies $(76,77)$. These results demonstrated that Bacillus can indeed be part in the gut microbiota.

Supplementation with both $\mathrm{AX}$ and $\mathrm{XG}$ in maize-based SIEMP also showed higher 
reproducibility than only supplementing AX to maize-based SIEMP. Figure S7 shows that microbiota compositions between replicates at different time points upon AX-XG feeding was much more similar than those on AX. In order to test the reproducibility of microbial composition, C.V. of replicates of microbiota fed with AX-XG of alpha diversity, microbial abundance, and microbial activities were evaluated (Table S4, S5, and S6), which were all lower than $15 \%$ (expect for 5 taxa in microbial abundance). The low C.V.s indicated that the results of the biological replicates were very reproducible (78). The high reproducibility of TIM-2 (the human version) was previously evaluated by the clustering of the microbiota after the adaptation period (at t0) (79), and during an entire experiment (43). Since SLIM (or TIM2 ) is computer-controlled, it is highly reproducible. This has been shown in numerus studies $(5,25,26,43)$.

Overall, at the phylum, family, and genus levels, the microbial composition of AX-XG-fed microbiota was more similar to that of the original inoculum than those of AX-fed and maize starch-fed microbiota (Figure 7, Figure 8A), and the dominant phyla were Firmicutes and Bacteroidetes, which is in agreement with previous reports $(1,7,8)$. Even though the microbial composition of the microbiota fed with AX-XG or AX was not exactly the same as the original inoculum, their predictive functional profiles were similar to each other, and clustered together (Figure 8B). Other studies have shown that function structure across microbial community can be remarkably similar, although the taxonomic composition was highly variable $(2,80)$. This can be explained because many of the same functions can be contained within different species that have independently evolved (2). We also found that microbial composition of samples in AX-XG had less individual differences compared with that in AX ( Figure 7). And the swine in vitro large intestine model can be reproduced to contain similar microbial communities (table S4, S5, and S6). All the data collectively together, we therefore conclude that both AX and XG were recommended to be supplemented to maize starch based SIEMP, and that the pig gut microbiota fed with this optimized SIEMP had a similar microbial composition and functional profile as the original inoculum.

\subsection{Conclusion}

In conclusion, different sources of starch significantly changed the pig microbiota composition, ecological network, and predictive functional profile in vitro, while two nonstarch polysaccharides did not affect this further. In study I, the predicted functional profile of the maize starch group was more similar to the original pig fecal sample, whereas in addition in study II, both the microbial composition and the predictive functional profile of AX-XG groups were more similar to the original swine fecal samples, compared with maize starch and AX alone. Study II showed that the pig microbiota fed with SIEMP (comprising of maize starch, $\mathrm{AX}, \mathrm{XG}$, and the rest of the compounds in normal SIEM) were highly reproducible. All data collectively considered, maize starch with arabinoxylans (AX) and xyloglucan (XG) should be added to the SIEM recipe to simulate ileal effluent medium for pigs (SIEMP), and this research lays the foundation for experiments mimicking the swine large intestine fermentation, and the possibility to establish the effect of feed interventions on this activity, e.g. with respect to whole intestinal energy extraction of feedstuffs. 
Chapter 2 
Reference:

1. Ramayo-Caldas Y, Mach N, Lepage P, Levenez F, Denis C, Lemonnier G, et al. Phylogenetic network analysis applied to pig gut microbiota identifies an ecosystem structure linked with growth traits. The ISME journal. 2016;10(12):2973.

2. Lozupone CA, Stombaugh JI, Gordon JI, Jansson JK, Knight R. Diversity, stability and resilience of the human gut microbiota. Nature. 2012;489(7415):220.

3. Kraler M, Ghanbari M, Domig KJ, Schedle K, Kneifel W. The intestinal microbiota of piglets fed with wheat bran variants as characterised by $16 \mathrm{~S}$ rRNA next-generation amplicon sequencing. Arch Anim Nutr. 2016;70(3):173-89.

4. Warren FJ, Fukuma NM, Mikkelsen D, Flanagan BM, Williams BA, Lisle AT, et al. Food Starch Structure Impacts Gut Microbiome Composition. mSphere. 2018;3(3).

5. Martinez RC, Cardarelli HR, Borst W, Albrecht S, Schols H, Gutiérrez OP, et al. Effect of galactooligosaccharides and Bifidobacterium animalis Bb-12 on growth of Lactobacillus amylovorus DSM 16698, microbial community structure, and metabolite production in an in vitro colonic model set up with human or pig microbiota. FEMS Microbiol Ecol. 2013;84(1):110-23.

6. Hopkins MJ, Englyst HN, Macfarlane S, Furrie E, Macfarlane GT, McBain AJ. Degradation of crosslinked and non-cross-linked arabinoxylans by the intestinal microbiota in children. Appl Environ Microbiol. 2003;69(11):6354-60.

7. Casellas NM, Berri EM, Fabrellas JE, Levenez F, Lemonnier G, Denis C, et al., editors. Establishment of the swine gut microbiome during early life and further impact on adult health and performance. 34 International Society for Animal Genetics Conference; 2014.

8. Costa MO, Chaban B, Harding JCS, Hill JE. Characterization of the fecal microbiota of pigs before and after inoculation with "Brachyspira hampsonii". PLoS One. 2014;9(8):e106399.

9. Cantu-Jungles TM, do Nascimento GE, Zhang X, Iacomini M, Cordeiro LM, Hamaker BR. Soluble xyloglucan generates bigger bacterial community shifts than pectic polymers during in vitro fecal fermentation. Carbohydr. Polym. 2019;206:389-95.

10. Umu OC, Frank JA, Fangel JU, Oostindjer M, da Silva CS, Bolhuis EJ, et al. Resistant starch diet induces change in the swine microbiome and a predominance of beneficial bacterial populations. Microbiome. 2015;3:16.

11. Niu Q, Li P, Hao S, Zhang Y, Kim SW, Li H, et al. Dynamic distribution of the gut microbiota and the relationship with apparent crude fiber digestibility and growth stages in pigs. Sci Rep. 2015;5:9938.

12. Belobrajdic DP, Bird AR, Conlon MA, Williams BA, Kang S, McSweeney CS, et al. An arabinoxylanrich fraction from wheat enhances caecal fermentation and protects colonocyte DNA against diet-induced damage in pigs. Br. J. Nutr. 2012;107(9):1274-82.

13. Minekus M, Marteau P, Havenaar R. Multicompartmental dynamic computer-controlled model simulating the stomach and small intestine. Altern Lab Anim. 1995.

14. Molly K, Woestyne MV, Verstraete W. Development of a 5-step multi-chamber reactor as a simulation of the human intestinal microbial ecosystem. Appl. Microbiol. Biotechnol. 1993;39(2):254-8.

15. Barroso E, Cueva C, Peláez C, Carmen M. The computer-controlled multicompartmental dynamic model of the gastrointestinal system SIMGI. The Impact of Food Bioactives on Health. 2015:319.

16. Barry J, Hoebler C, Macfarlane G, Macfarlane S, Mathers J, Reed K, et al. Estimation of the fermentability of dietary fibre in vitro: a European interlaboratory study. Br. J. Nutr. 1995;74(3):303-22.

17. Venema K, Van den Abbeele P. Experimental models of the gut microbiome. Best Pract Res Cl Ga. 2013;27(1):115-26.

18. Minekus M, Smeets-Peeters M, Bernalier A, Marol-Bonnin S, Havenaar R, Marteau P, et al. A computercontrolled system to simulate conditions of the large intestine with peristaltic mixing, water absorption and absorption of fermentation products. Appl Microbiol Biotechnol. 1999;53(1):108-14.

19. Barroso E, Cueva C, Peláez C, Martínez-Cuesta MC, Requena T. Development of human colonic microbiota in the computer-controlled dynamic SIMulator of the GastroIntestinal tract SIMGI. LWT-Food Sci Technol. 2015;61(2):283-9.

20. Gibson GR, Cummings JH, Macfarlane GT. Use of a three-stage continuous culture system to study the effect of mucin on dissimilatory sulfate reduction and methanogenesis by mixed populations of human gut bacteria. Appl Environ Microbiol. 1988;54(11):2750-5.

21. Macfarlane G, Cummings J, Macfarlane S, Gibson G. Influence of retention time on degradation of pancreatic enzymes by human colonic bacteria grown in a 3-stage continuous culture system. J. Appl. Microbiol. 1989;67(5):521-7.

22. Venema K, Nuenen Mv, Smeets-Peeters M, Minekus M, Havenaar R. TNO's in vitro large intestinal model: an excellent screening tool for functional food and pharmaceutical research. Ernährung. 2000;24(12):55864.

23. Binsl T, De Graaf A, Venema K, Heringa J, Maathuis A, De Waard P, et al. Measuring non-steady-state 
metabolic fluxes in starch-converting faecal microbiota in vitro. Benef microbes. 2010;1(4):391-405.

24. Maathuis AJ, van den Heuvel EG, Schoterman MH, Venema K. Galacto-oligosaccharides have prebiotic activity in a dynamic in vitro colon model using a (13)C-labeling technique. J Nutr. 2012;142(7):1205-12.

25. Kovatcheva-Datchary P, Egert M, Maathuis A, Rajilić-Stojanović M, De Graaf AA, Smidt H, et al. Linking phylogenetic identities of bacteria to starch fermentation in an in vitro model of the large intestine by RNAbased stable isotope probing. Environ Microbiol. 2009;11(4):914-26.

26. Rose DJ, Venema K, Keshavarzian A, Hamaker BR. Starch-entrapped microspheres show a beneficial fermentation profile and decrease in potentially harmful bacteria during in vitro fermentation in faecal microbiota obtained from patients with inflammatory bowel disease. Br. J. Nutr. 2010;103(10):1514-24.

27. Maathuis A, Hoffman A, Evans A, Sanders L, Venema K. The effect of the undigested fraction of maize products on the activity and composition of the microbiota determined in a dynamic in vitro model of the human proximal large intestine. J Am Coll Nutr. 2009;28(6):657-66.

28. de Souza CB, Roeselers G, Troost F, Jonkers D, Koenen M, Venema K. Prebiotic effects of cassava bagasse in TNO's in vitro model of the colon in lean versus obese microbiota. J. Funct. Foods. 2014;11:210-20.

29. Klindworth A, Pruesse E, Schweer T, Peplies J, Quast C, Horn M, et al. Evaluation of general 16S ribosomal RNA gene PCR primers for classical and next-generation sequencing-based diversity studies. Nucleic Acids Res. 2013;41(1):e1-e.

30. Callahan BJ, McMurdie PJ, Rosen MJ, Han AW, Johnson AJA, Holmes SP. DADA2: High-resolution sample inference from Illumina amplicon data. Nat. Methods. 2016;13:581.

31. Lozupone CA, Hamady M, Kelley ST, Knight R. Quantitative and qualitative beta diversity measures lead to different insights into factors that structure microbial communities. Appl Environ Microbiol. 2007;73(5):1576-85.

32. Anderson MJ. A new method for non-parametric multivariate analysis of variance. Austral Ecol. 2001;26(1):32-46

33. Bokulich NA, Kaehler BD, Rideout JR, Dillon M, Bolyen E, Knight R, et al. Optimizing taxonomic classification of marker-gene amplicon sequences with QIIME 2's q2-feature-classifier plugin. Microbiome. 2018;6(1):90.

34. Faust K, Sathirapongsasuti JF, Izard J, Segata N, Gevers D, Raes J, et al. Microbial co-occurrence relationships in the human microbiome. PLoS Comp Biol. 2012;8(7):e1002606.

35. Assenov Y, Ramírez F, Schelhorn S-E, Lengauer T, Albrecht M. Computing topological parameters of biological networks. Bioinformatics. 2007;24(2):282-4.

36. Shannon P, Markiel A, Ozier O, Baliga NS, Wang JT, Ramage D, et al. Cytoscape: a software environment for integrated models of biomolecular interaction networks. Genome Res. 2003;13(11):2498-504

37. Bader GD, Hogue CW. An automated method for finding molecular complexes in large protein interaction networks. BMC bioinformatics. 2003;4(1):2.

38. Segata N, Izard J, Waldron L, Gevers D, Miropolsky L, Garrett WS, et al. Metagenomic biomarker discovery and explanation. Genome biology. 2011;12(6):R60.

39. Langille MG, Zaneveld J, Caporaso JG, McDonald D, Knights D, Reyes JA, et al. Predictive functional profiling of microbial communities using 16S rRNA marker gene sequences. Nat. Biotechnol. 2013;31(9):814.

40. Parks DH, Tyson GW, Hugenholtz P, Beiko RG. STAMP: statistical analysis of taxonomic and functional profiles. Bioinformatics. 2014;30(21):3123-4.

41. Rehman A, Heinsen FA, Koenen ME, Venema K, Knecht H, Hellmig S, et al. Effects of probiotics and antibiotics on the intestinal homeostasis in a computer controlled model of the large intestine. BMC Microbiol. 2012;12:47.

42. Aguirre M, Jonkers DM, Troost FJ, Roeselers G, Venema K. In vitro characterization of the impact of different substrates on metabolite production, energy extraction and composition of gut microbiota from lean and obese subjects. PLoS One. 2014;9(11):e113864.

43. Aguirre M, de Souza CB, Venema K. The gut microbiota from lean and obese subjects contribute differently to the fermentation of arabinogalactan and inulin. PloS one. 2016;11(7):e0159236.

44. Gao K, Xu A, Krul C, Venema K, Liu Y, Niu Y, et al. Of the major phenolic acids formed during human microbial fermentation of tea, citrus, and soy flavonoid supplements, only 3, 4-dihydroxyphenylacetic acid has antiproliferative activity. Nutr. J. 2006;136(1):52-7.

45. Pluske J, Hampson D. Rice-based Diets in Pigs: For Protection Against Intestinal Bacterial Infections: a Report for the Rural Industries Research and Development Corporation: Rural Industries Research and Development Corporation; 2005.

46. Jackson PG, Cockcroft PD. Handbook of pig medicine: Elsevier Health Sciences; 2007

47. Cummings J, Macfarlane G. The control and consequences of bacterial fermentation in the human colon. J. Appl. Microbiol. 1991;70(6):443-59.

48. Baghurst PA, Baghurst KI, Record SJ. Dietary fibre, non-starch polysaccharides and resistant starch: a 
review. Food Australia. 1996;48(3):S3-5.

49. Englyst HN, Kingman SM, Hudson GJ, Cummings JH. Measurement of resistant starch in vitro and in vivo. Br. J. Nutr. 1996;75(5):749-55

50. Svihus B, Uhlen AK, Harstad OM. Effect of starch granule structure, associated components and processing on nutritive value of cereal starch: A review. Anim. Feed Sci. Technol. 2005;122(3):303-20.

51. Bird A, Illman R, Hayakawa T, Topping D, editors. Brown rice increases faecal and large bowel shortchain fatty acid levels in pigs. Proceedings-Nutrition Society of Australia; 1997: 21:135-135.

52. Kleessen B, Sykura B, Zunft H-J, Blaut M. Effects of inulin and lactose on fecal microflora, microbial activity, and bowel habit in elderly constipated persons. Am J Clin Nutr . 1997;65(5):1397-402.

53. Martínez I, Kim J, Duffy PR, Schlegel VL, Walter J. Resistant starches types 2 and 4 have differential effects on the composition of the fecal microbiota in human subjects. PloS one. 2010;5(11):e15046.

54. Sajilata MG, Singhal RS, Kulkarni PR. Resistant starch-a review. Comprehensive reviews in food science and food safety. Compr. Rev. Food Sci. Food Saf. 2006;5(1):1-17.

55. Faust K, Raes J. Microbial interactions: from networks to models. Nat. Rev. Microbiol. 2012;10:538.

56. Cohen R, Havlin S. Complex networks: structure, robustness and function: Cambridge university press; 2010.

57. Turnbaugh PJ, Ley RE, Mahowald MA, Magrini V, Mardis ER, Gordon JI. An obesity-associated gut microbiome with increased capacity for energy harvest. nature. 2006;444(7122):1027.

58. Turnbaugh PJ, Hamady M, Yatsunenko T, Cantarel BL, Duncan A, Ley RE, et al. A core gut microbiome in obese and lean twins. nature. 2009;457(7228):480.

59. Bervoets L, Van Hoorenbeeck K, Kortleven I, Van Noten C, Hens N, Vael C, et al. Differences in gut microbiota composition between obese and lean children: a cross-sectional study. Gut Pathog. 2013;5(1):10.

60. Brouns F, Arrigoni E, Langkilde AM, Verkooijen I, Fässler C, Andersson H, et al. Physiological and Metabolic Properties of a Digestion-Resistant Maltodextrin, Classified as Type 3 Retrograded Resistant Starch. J. Agric. Food Chem. 2007;55(4):1574-81.

61. Gaenzle MG. Lactic metabolism revisited: metabolism of lactic acid bacteria in food fermentations and food spoilage. Curr Opin Food Sci. 2015;2:106-17.

62. David LA, Maurice CF, Carmody RN, Gootenberg DB, Button JE, Wolfe BE, et al. Diet rapidly and reproducibly alters the human gut microbiome. Nature. 2014;505(7484):559-63.

63. Duncan SH, Belenguer A, Holtrop G, Johnstone AM, Flint HJ, Lobley GE. Reduced dietary intake of carbohydrates by obese subjects results in decreased concentrations of butyrate and butyrate-producing bacteria in feces. Appl Environ Microbiol. 2007;73(4):1073-8.

64. Neyrinck AM, Possemiers S, Druart C, Van de Wiele T, De Backer F, Cani PD, et al. Prebiotic effects of wheat arabinoxylan related to the increase in bifidobacteria, Roseburia and Bacteroides/Prevotella in diet-induced obese mice. PloS one. 2011;6(6):e20944.

65. Stevenson DM, Weimer PJ. Dominance of Prevotella and low abundance of classical ruminal bacterial species in the bovine rumen revealed by relative quantification real-time PCR. Appl. Microbiol. Biotechnol. 2007;75(1):165-74.

66. Jami E, Mizrahi I. Composition and similarity of bovine rumen microbiota across individual animals. PloS one. 2012;7(3):e33306.

67. De Menezes AB, Lewis E, O'Donovan M, O'Neill BF, Clipson N, Doyle EM. Microbiome analysis of dairy cows fed pasture or total mixed ration diets. FEMS Microbiol Ecol. 2011;78(2):256-65.

68. Tian D, Xu X, Peng Q, Wen Z, Zhang Y, Wei C, et al. In vitro fermentation of arabinoxylan from oat (Avena sativa L.) by Pekin duck intestinal microbiota. 3 Biotech. 2019;9(2):54.

69. Emerson EL, Weimer PJ. Fermentation of model hemicelluloses by Prevotella strains and Butyrivibrio fibrisolvens in pure culture and in ruminal enrichment cultures. Appl. Microbiol. Biotechnol. 2017;101(10):426978.

70. Seedorf H, Griffin NW, Ridaura VK, Reyes A, Cheng J, Rey FE, et al. Bacteria from diverse habitats colonize and compete in the mouse gut. Cell. 2014;159(2):253-66.

71. Ormerod KL, Wood DLA, Lachner N, Gellatly SL, Daly JN, Parsons JD, et al. Genomic characterization of the uncultured Bacteroidales family S24-7 inhabiting the guts of homeothermic animals. Microbiome. 2016;4(1):36.

72. Larsbrink J, Rogers TE, Hemsworth GR, McKee LS, Tauzin AS, Spadiut O, et al. A discrete genetic locus confers xyloglucan metabolism in select human gut Bacteroidetes. Nature. 2014;506(7489):498.

73. Svartström O, Alneberg J, Terrapon N, Lombard V, de Bruijn I, Malmsten J, et al. Ninety-nine de novo assembled genomes from the moose (Alces alces) rumen microbiome provide new insights into microbial plant biomass degradation. The ISME journal. 2017;11(11):2538.

74. $\quad$ Scheller HV, Ulvskov P. Hemicelluloses. Annu Rev Plant Biol. 2010;61:263-89.

75. Spinosa MR, Braccini T, Ricca E, De Felice M, Morelli L, Pozzi G, et al. On the fate of ingested Bacillus 
spores. Res Microbiol. 2000;151(5):361-8.

76. Adami A, Cavazzoni V. Occurrence of selected bacterial groups in the faeces of piglets fed with Bacillus coagulans as probiotic. Journal of Basic Microbiology: An International Journal on Biochemistry, Physiology, Genetics, Morphology, and Ecology of Microorganisms. J. Basic Microbiol. 1999;39(1):3-9.

77. Turnbull P, Kramer J. Intestinal carriage of Bacillus cereus: faecal isolation studies in three population groups. Epidemiol Infect. 1985;95(3):629-38.

78. Gomez KA, Gomez AA. Statistical procedures for agricultural research: John Wiley \& Sons; 1984.

79. Verhoeckx K, Cotter P, López-Expósito I, Kleiveland C, Lea T, Mackie A, et al. The impact of food bioactives on health: in vitro and ex vivo models: Springer; 2015.

80. Louca S, Jacques SM, Pires AP, Leal JS, Srivastava DS, Parfrey LW, et al. High taxonomic variability despite stable functional structure across microbial communities. Nat. Ecol. Evol. 2017;1(1):0015. 


\section{Supplementary Figures:}

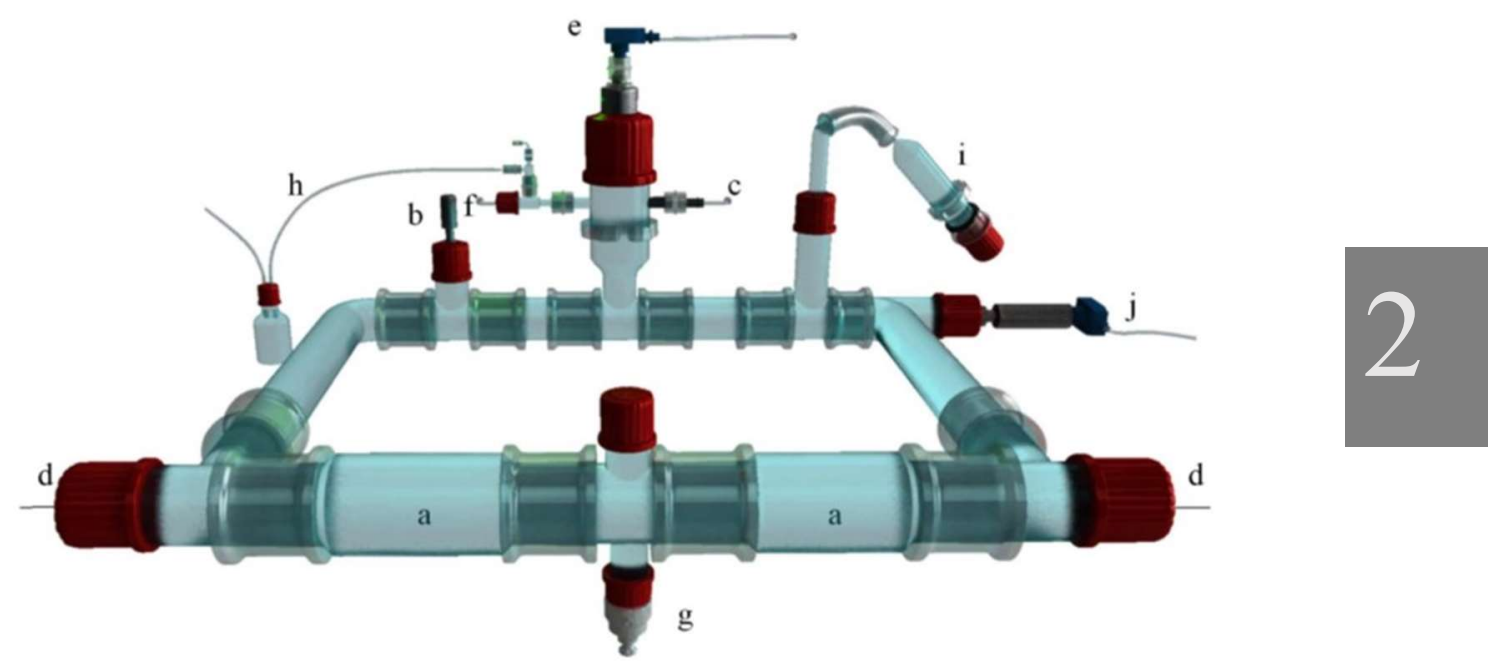

Figure S1. SLIM schematic, modified from Minekus et al. (1999). a) peristaltic compartments with a hollow fiber membrane inside, b) $\mathrm{pH}$ sensor, c) $\mathrm{NaOH}$ secretion, d) dialysate system, e) level sensor, f) gaseous $\mathrm{N}_{2}$ inlet, g) sampling port, h) gas outlet, i) test compound + SIEM feeding syringe, $\mathrm{j}$ ) temperature sensor. 


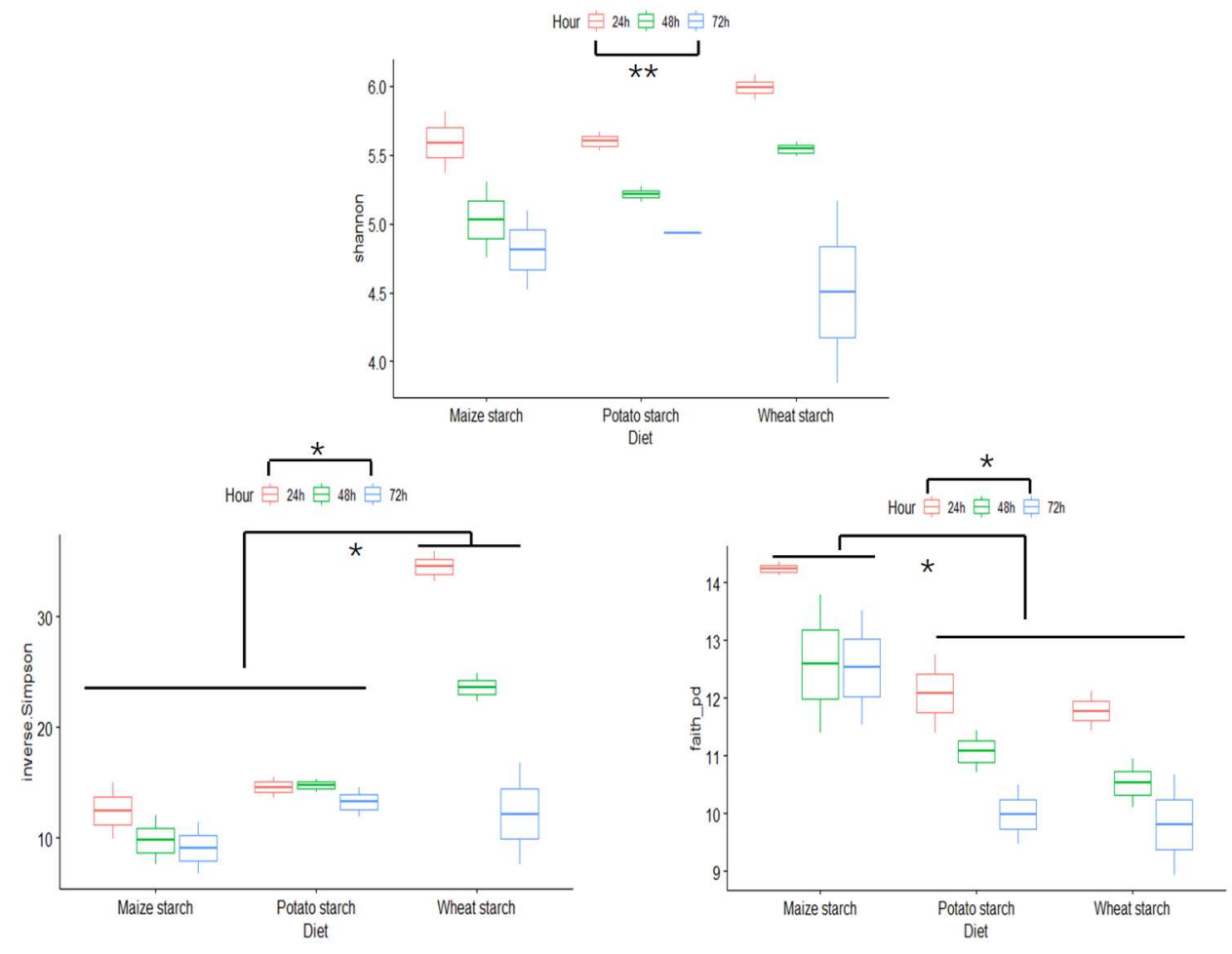

Supplementary Figure S2. The effect of starch source and fermentation time on alpha diversity indices (Shannon, inverse Simpson and Faith_PD).
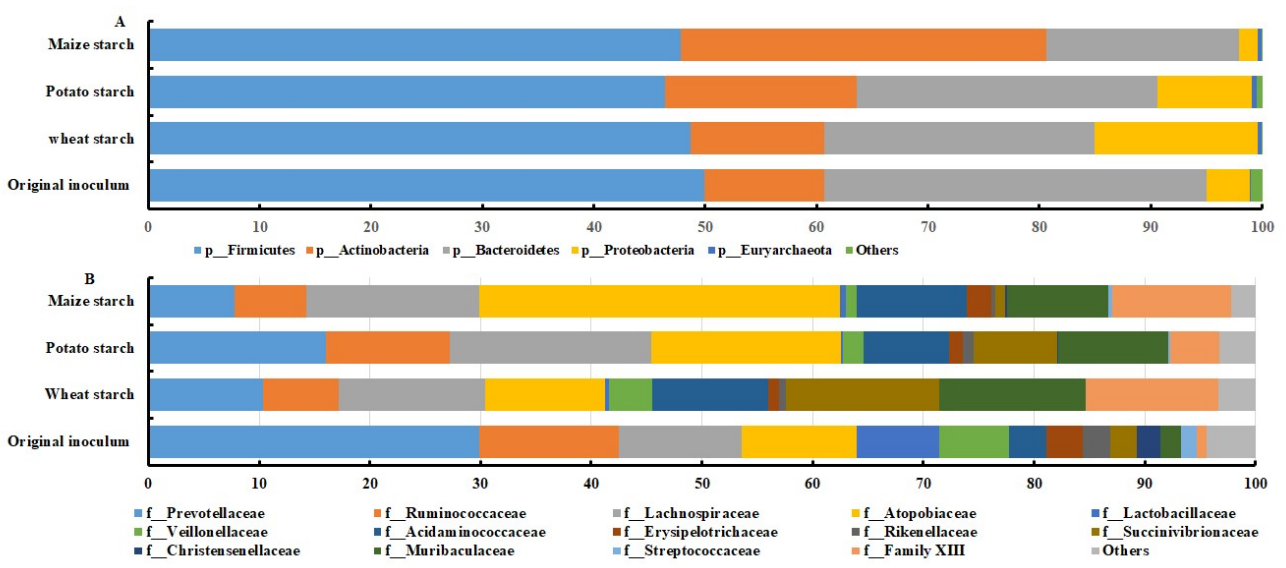

Figure S3: Relative abundances of microbial phyla (A) and families (B) in the original 
inoculum, and microbiota fed with different starches

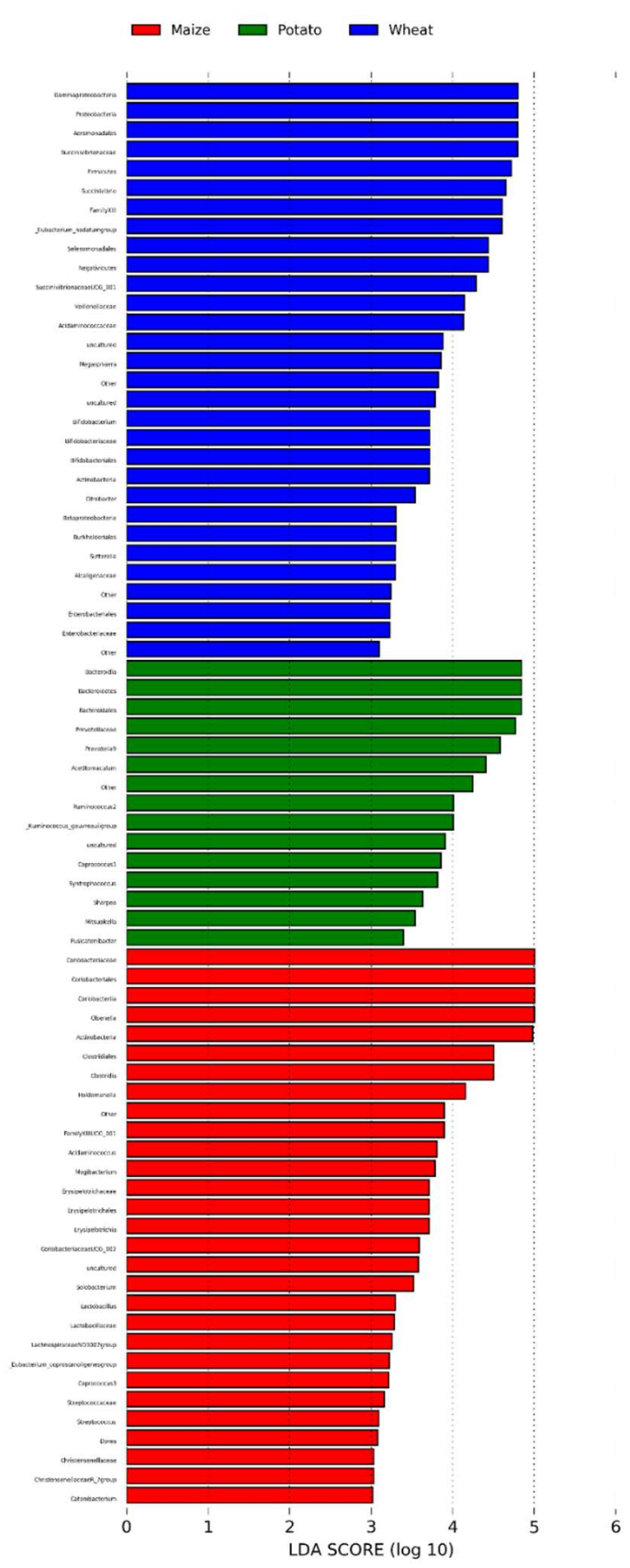

Supplementary Figure S4. LEfSe results on the microbiome of Maize-, Potato- and Wheatstarch fed groups. Histogram of the LDA scores computed for features differentially abundant 
among maize, potato, and wheat starch treated microbiotas.
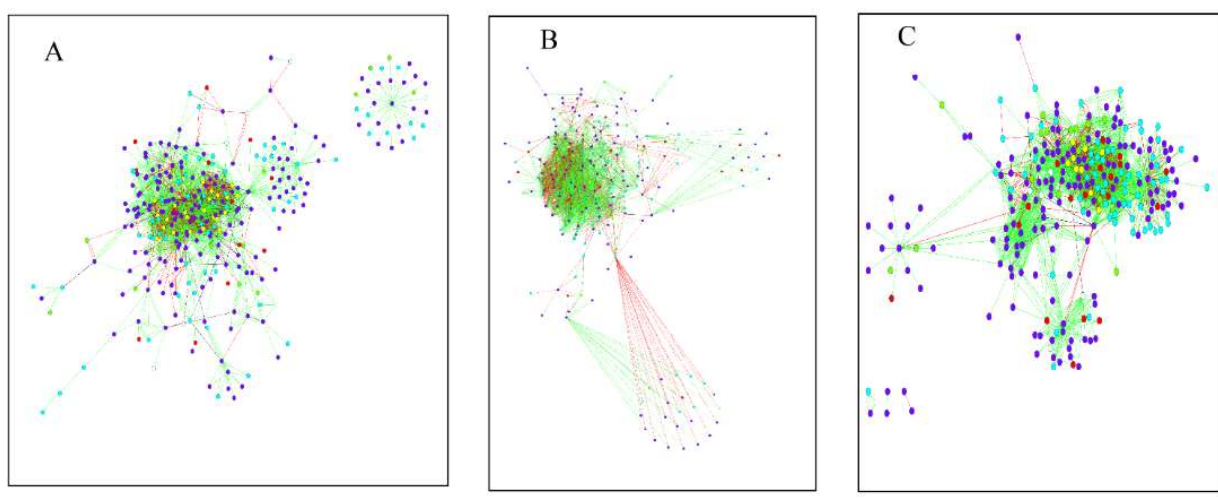

Supplementary Figure S5. Co-occurrence networks in the porcine gut microbiome. A. Maize-starch-based network. B. Potato-starch-based network. C. Wheat-starch-based network. Modules were detected by using MCODE in Cytoscape. Each color represents a module. Green edges represent positive interaction, while red ones represent negative interaction, and black ones are unknown.

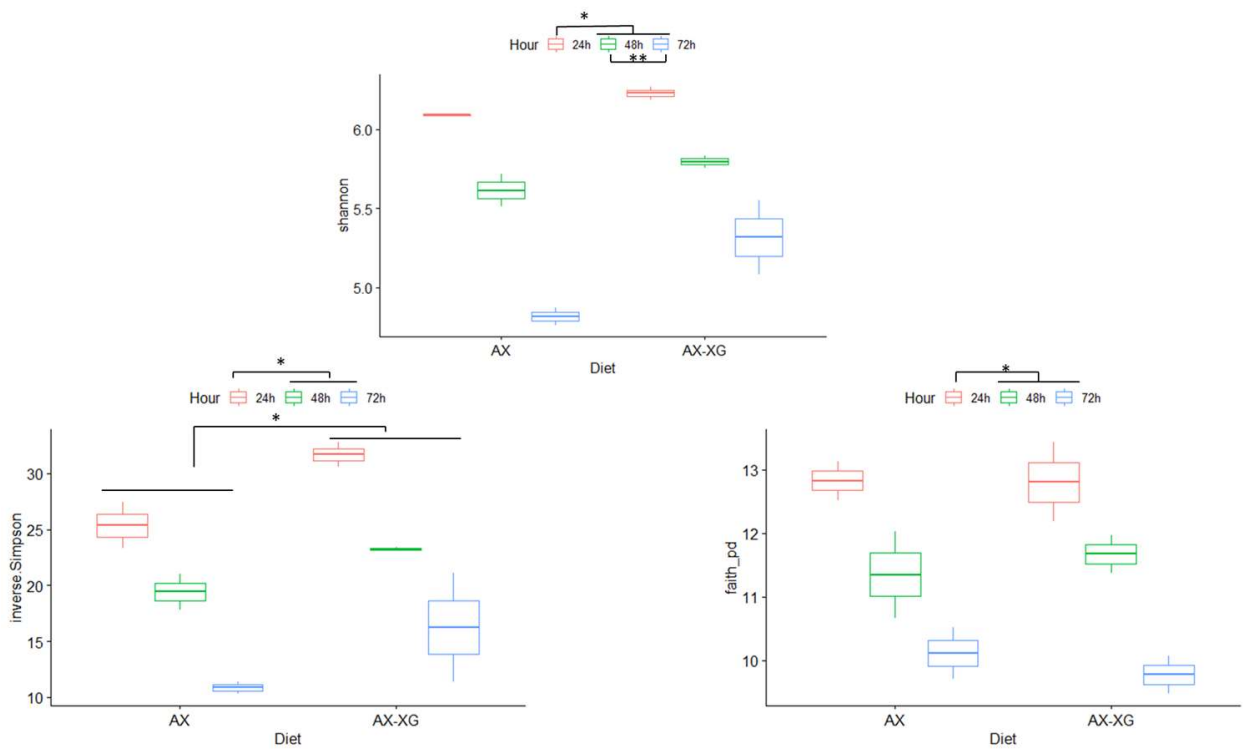

Supplementary Figure S6. The effect of non-starch polysaccharides and fermentation time on alpha diversity indices (Shannon, inverse Simpson and Faith_PD). 

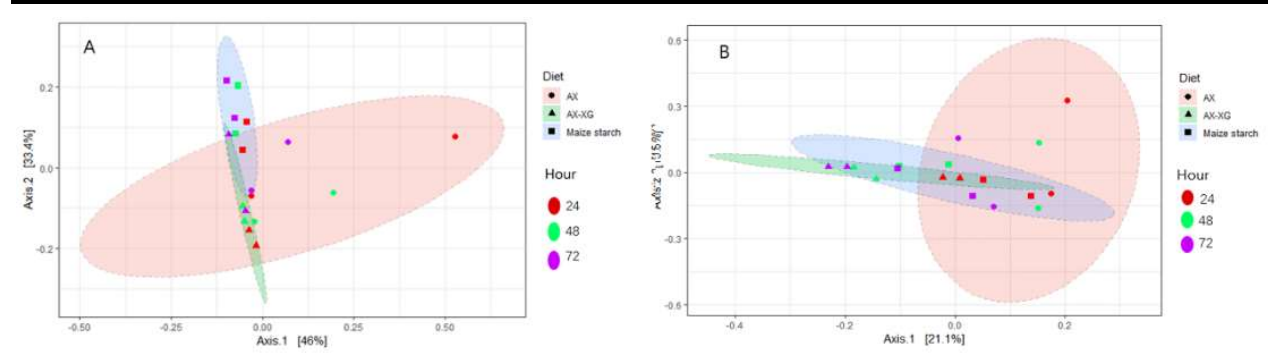

Figure S7. Principal coordinate analysis ( $\mathrm{PCoA}$ ) plots generated based on the calculated distances in a weighted (A) and unweighted (B) UniFrac matrix. Samples were grouped by color (time-point) and shape (in terms of diet group) they belonged to. The ellipses were drawn at the 0.95 confidence level. Weighted: AX to AX-XG $(P=0.003), \mathrm{AX}-\mathrm{XG}$ to maize starch $(P=0.003)$, AX to maize starch $(P=0.003)$; Unweighted: AX to AX-XG $(P=0.034)$, AX-XG to maize starch $(P=0.024)$, AX to maize starch $(P=0.141)$.

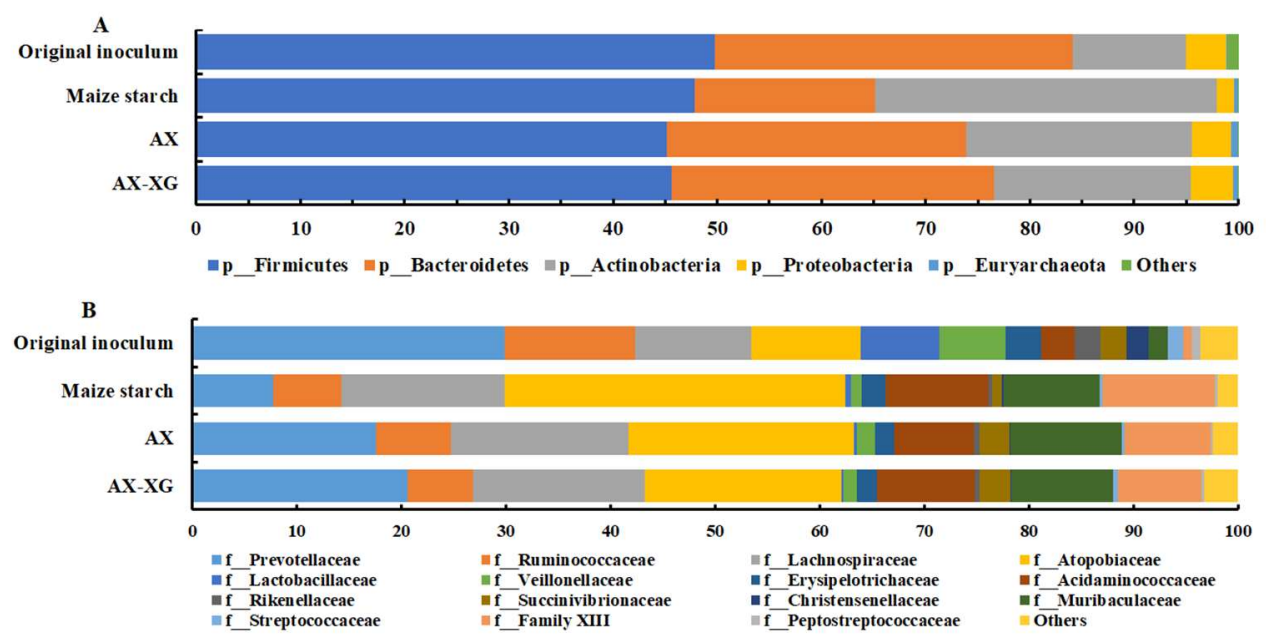

Figure S8: Relative abundances of microbial phyla and families in the original inoculum and microbiota fed with maize starch, AX, and AX-XG. 
A

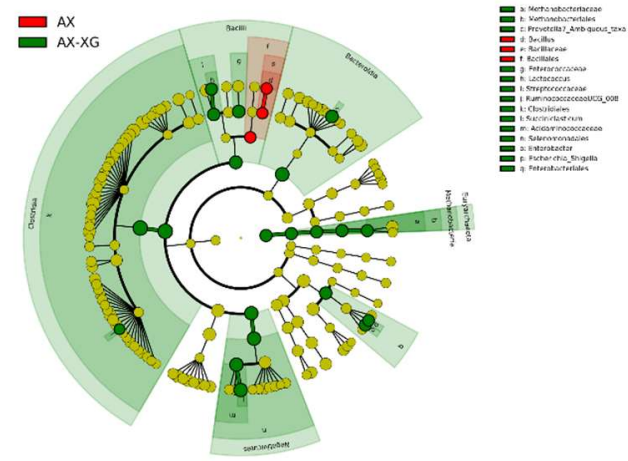

B

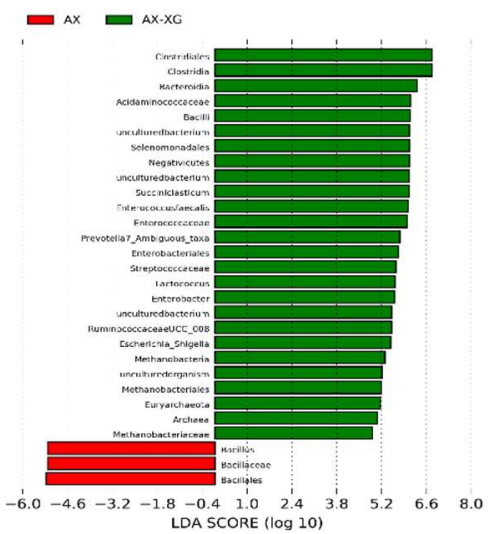

Supplementary Figure S9. LEfSe results on the microbiome of AX and AX-XG group. (A) Histogram of the LDA scores computed for features differentially abundant between AX and AX-XG group. (B) Taxonomic representation of statistically and biologically consistent differences between AX and AX-XG groups. Differences in the most abundance taxa are indicated, red indicating significantly higher in the AX group, green indicating significantly higher in the AX-XG group. 


\title{
Chapter 3
}

\section{Pretreatment of rapeseed meal increases its recalcitrant fibre fermentation and alters the microbial community in an in vitro model of swine large intestine}

\author{
Cheng Long ${ }^{1,2}$, Koen Venema ${ }^{1,2^{*}}$ \\ ${ }^{1}$ Faculty of Science and Engineering, Centre for Healthy Eating \& Food Innovation, \\ Maastricht University - campus Venlo, The Netherlands \\ ${ }^{2}$ School of Nutrition and Translational Research in Metabolism (NUTRIM), Maastricht \\ University, The Netherlands \\ *Correspondence: \\ Maastricht University - campus Venlo, Centre for Healthy Eating \& Food Innovation, St. \\ Jansweg 20, 5928 RC Venlo, The Netherlands \\ k.venema@maastrichtuniversity.nl; tel.: +31622435111
}

Accepted for publication in Frontiers in Microbiology. 


\begin{abstract}
The aim of current study was to investigate whether degradation of rapeseed meal (RSM) which was modified by a cellulase, two pectinase, or alkaline treatment was improved by the swine gut microbiota compared to untreated RSM, and whether the microbiota composition was changed. An in vitro study was performed to assess how enzymatic and chemical pretreated rapeseed meal (RSM) influences the fibre fermentation and microbial community in the swine large intestine. RSM was processed enzymatically by a cellulase (CELL), two pectinases (PECT), or chemically by an alkaline (ALK) treatment. 16S rRNA gene sequencing data was performed to evaluate changes in the gut microbiota composition, whereas short chain fatty acid production (ion-chromatography) and non-starch polysaccharides (NSP) composition (using monoclonal antibodies; mAbs) were used to assess fibre degradation. The results showed that ALK, CELL, PECT1, and PECT2 changed microbial community composition, increased the abundance of microbial fibre-degrading enzymes and pathways, and increased acetic acid, propionic acid, butyric acid, and total SCFA production. The increased genera also positively correlated with SCFA production. The cell wall polysaccharide structures of RSM shifted after ALK, CELL, PECT1, and PECT2 treatment. The degradation of NSP during the fermentation period was dynamic, and not continuous based on the epitope recognition by mAbs. This study provides the first detailed analysis of changes in the swine intestinal microbiota due to RSM modified by ALK, CELL, PECT1 and PECT2. ALK, CELL, PECT1 and PECT2 altered microbial community structure, shifted the predicted functional metagenomic profile and subsequently increased total SCFA production. Our findings that ALK, CELL, PECT1 and PECT2 increased fiber degradability in RSM could help guide feed additive strategies to improve efficiency and productivity in swine industry. The current study gave insight into how feed enzyme modulate microbial status, which provides good opportunity to develop novel carbohydrase, particularly in swine feed.
\end{abstract}




\section{Introduction}

Rapeseed meal (RSM), a by-product of rapeseed oil production, is not only a suitable protein source for swine feed but also a potentially energy source. RSM contains 20 to 40 \% nonstarch polysaccharides (NSP) $(1,2)$. The primary cell walls of RSM consist of pectin and xyloglucan and its cellulose microfibrils are interlinked with xyloglucan via hydrogen bonds forming a stiff network (3). Pectins are linked to each other and cross-linked between pectin and hemicellulose, and between pectin and cellulose (4). Pectins, consisting of homogalacturonan, rhamnogalacturonan, xylogalacturonan, arabinogalactan and arabinan, are the major polysaccharides present in the dehulled rapeseed meal (5). In the secondary cell wall of RSM, the main carbohydrates are 4-O-methyglucuronoxylan, xyloglucan, and cellulose. A drawback of using RSM in animal feed is that the complex cell wall polysaccharides cannot be utilized by endogenous enzymes from monogastric animals (e.g. pigs), and also can only partly be fermented by the microbial community in the gastrointestinal tract (GIT) of the pig.

Therefore, the animal feed sector seeks opportunities to enhance degradability of NSP of feedstuffs, in order to improve its potential as a nutrient source for domestic animals. Previous research showed that physical processing technologies, such as hammer milling, pelleting, wet-milling, extrusion, and mild hydrothermal acid treatment, had limited effect on recalcitrant NSP structures $(6,7)$. As a result, more efficient solutions are needed to modify the cell wall architecture and allow the gut microbiota to utilize the complex carbohydrates. Former research has shown that NSP-degrading enzymes, such as cellulase and pectinases, could open the cell wall structure and improve NSP degradability $(8,9)$. Previous studies showed that addition of pectolytic enzymes improved degradability of NSP of RSM in vitro (10) and in broilers $(11,12)$. However, none of the above studies investigated how the gut microbiota was affected by the modified RSM or by the feed enzymes. It is important to know this, as NSP can only be fermented by microbes. Bindelle et al. (13) demonstrated that NSP-degrading enzymes increased abundances of cellulolytic Ruminococcus- and xylanolytic Clostridium-like bacteria and altered fermentation patterns of barley cultivars and wheat products. Torok and colleagues (14) investigated changes in gut microbial population in response to the supplementation of an NSP-degrading enzyme (containing $\beta$-glucanase, xylanase, and protease activities) in a barley-based diet in chickens, and the results showed that microbial composition revealed distinct clusters correlating with un-supplemented and enzyme supplemented birds. Previous research reported that the pretreatment of feed stuffs with carbohydrases can cause the release of reducing sugars and other hydrolysis materials, promoting chemotactic response in specific bacteria, and stimulating their attachment to feed particles, and thereby growth of these microbes $(9,15-$ 17).

In the present study, RSM (predigested with digestive enzymes) was treated independently with two kinds of pectinases (PECT1 and PECT2), one cellulase (CELL), or alkaline (ALK), and afterwards the untreated and treated RSM preparations were fermented in the Swine Large Intestine in vitro Model (SLIM) (18). The aim of the current study was to investigate whether fermentation by the swine gut microbiota of treated RSM was improved compared to untreated RSM, and whether the microbiota composition and activity were changed. 


\section{Materials and methods}

\section{Substrate preparation}

Rapeseed meal (Brassica napus, Cargill N.V., Antwerp, Belgium; 2011) was obtained from a commercial feed mill (Agrifirm B.V., Utrecht, the Netherlands). Preparation method I (predigestion of RSM after carbohydrase or alkaline treatment) [Figure 1]: to $200 \mathrm{~g}$ of RSM

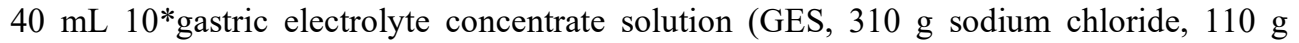
potassium chloride, $15 \mathrm{~g}$ calcium chloride di-hydrate, and $4840 \mathrm{~g}$ ultrapure water) and 360 $\mathrm{mL}$ ultrapure water were added. The $\mathrm{pH}$ was adjusted to 5.5 and then nothing (CON), $10 \mathrm{~mL}$ of alkaline (ALK, $6 \mathrm{M} \mathrm{NaOH}$ ), or the following carbohydrases were added CELL (Accellerase 1000, Sigma-Aldrich, Missouri, United States), PECT1 (Pectinex Ultra SP, Novozymes A/S, Bagsvaerd, Denmark), or PECT2 (Multifect Pectinase, DuPont Industrial Biosciences, Genencor division, Rochester, NY). Enzyme preparations were incubated at $37^{\circ} \mathrm{C}$ for $2 \mathrm{~h}$, with occasional shaking (every $30 \mathrm{mins}$ ), while ALK was incubated overnight at $4{ }^{\circ} \mathrm{C}$. Enzyme preparations were then heated at $100{ }^{\circ} \mathrm{C}$ for $5 \mathrm{~min}$ to inactive enzymes. Afterwards, for all five samples, $120 \mathrm{~mL}$ GES was added and $\mathrm{pH}$ adjusted to 3 to continue with the gastric incubation according to the predigestion protocol as described elsewhere (19). After predigestion, the slurry was centrifuged $\left(8.000 \mathrm{~g}\right.$, at $4{ }^{\circ} \mathrm{C}$, for 20 minutes $)$ and dialysis was performed for the supernatants. For dialysis, a dialyzer (Sureflux, Nipro Europe Group Companies, Mechelen, Belgium) was used with a peristaltic pump to remove small digestion products and water. After reduction of the total volume to $\sim 450-500 \mathrm{~mL}$, supernatant was mixed with pellet, and freeze-dried. Method II (predigestion of RSM before carbohydrase or alkaline treatment) [Figure 1]: four batches of $200 \mathrm{~g}$ RSM were predigested as described

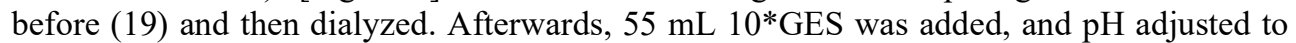
5.5, after which $10 \mathrm{~mL}$ of CELL, PECT1, PECT2, or ALK treatment commenced, respectively. Enzyme preparations were incubated at $37{ }^{\circ} \mathrm{C}$ for 2 hours with occasional shaking (every $30 \mathrm{mins}$ ), and ALK was incubated overnight at $4{ }^{\circ} \mathrm{C}$. Afterwards, enzyme preparations were heated at $100{ }^{\circ} \mathrm{C}$ for $5 \mathrm{~min}$ to inactive enzymes, and $\mathrm{pH}$ was neutralized to 6.5-7 with $\mathrm{HCl}$ or $\mathrm{NaOH}$, and the samples were freeze-dried. Samples are differentiated by the suffix _B (for before) or _A (for after) (e.g. PECT1_A) for carbohydrase- or ALKtreatment prior to and after digestion, respectively.

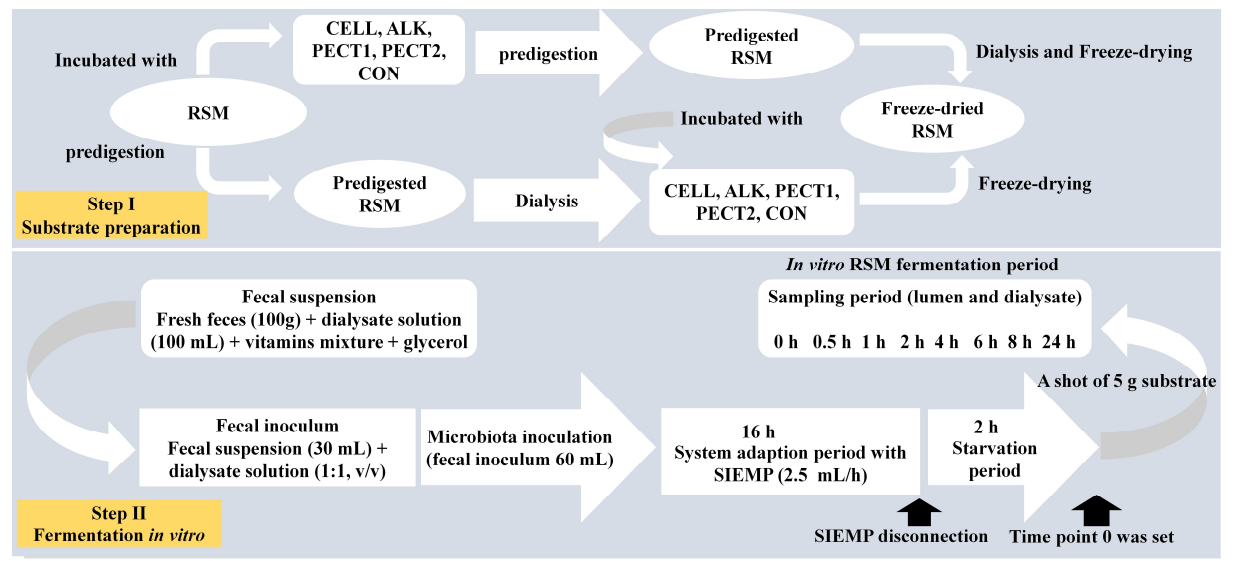

Figure 1. Schematic experimental setup for fibre fermentation in the Swine Large Intestine 
in vitro model (SLIM).

\section{Fermentation in The Swine in vitro Large Intestinal Model (SLIM)}

The setup of SLIM was as follows: a fully computer-controlled in vitro model based on TIM2 (20) was used to mimic the swine large intestine (18). The $\mathrm{pH}$ (5.9) was controlled by continuous addition of $2 \mathrm{M}$ sodium hydroxide. Standard ileal efflux medium of pigs (SIEMP) was used to simulate the materials entering the colon. The SIEMP, adapted from (21) and described in Long et al.(2020) (18) contained the following components (g/L): 74.6 maize starch, 9.0 xylan, 19.0 pectin, 9.0 amylopectin, 9.0 arabinogalactan, 9.0 arabinoxylan, 9.0 xyloglucan, 31.5 Tween $80,43.7$ casein, 0.7 ox-bile, 43.7 bactopepton, $4.7 \mathrm{~K}_{2} \mathrm{HPO}_{4} .3 \mathrm{H}_{2} \mathrm{O}$, $0.009 \mathrm{FeSO}_{4} .7 \mathrm{H}_{2} \mathrm{O}, 8.4 \mathrm{NaCl}, 0.8 \mathrm{CaCl}_{2} .2 \mathrm{H}_{2} \mathrm{O}, 0.7 \mathrm{MgSO}_{4} .7 \mathrm{H}_{2} \mathrm{O}, 0.05$ bile, 0.02 haemin and 0.3 cysteine $\cdot \mathrm{HCl}$, plus $1.5 \mathrm{~mL}$ of a vitamin mixture containing (per litre): $1 \mathrm{mg}$ menadione, $0.5 \mathrm{mg}$ vitamin B12, $2 \mathrm{mg}$ D-biotin, $10 \mathrm{mg}$ pantothenate, $5 \mathrm{mg}$ p-aminobenzoic, $4 \mathrm{mg}$ thiamine and $5 \mathrm{mg}$ nicotinamide acid. The $\mathrm{pH}$ was adjusted to 5.9. Dialysis liquid contained (per litre): $2.5 \mathrm{~g} \mathrm{~K}_{2} \mathrm{HPO}_{4} .3 \mathrm{H}_{2} \mathrm{O}, 0.005 \mathrm{~g} \mathrm{FeSO}_{4} .7 \mathrm{H}_{2} \mathrm{O}, 4.5 \mathrm{~g} \mathrm{NaCl}, 0.45 \mathrm{~g} \mathrm{CaCl}_{2} .2 \mathrm{H}_{2} \mathrm{O}, 0.05$ $\mathrm{g}$ bile, $0.5 \mathrm{~g} \mathrm{MgSO}_{4} .7 \mathrm{H}_{2} \mathrm{O}$ and $0.4 \mathrm{~g}$ cysteine $\cdot \mathrm{HCl}$, plus $1 \mathrm{~mL}$ of the vitamin mixture. All medium components were purchased at Tritium Microbiology (Eindhoven, The Netherlands). The pig fecal inoculum was a standardized microbiota from growing pigs, freshly collected from the floor (48 pens with 6 pigs/pen, Hypor Libra x Hypor Maxter, Hendrix Genetics, Boxmeer, The Netherlands), but only material from the top was selected (so not toughing the floor). Feces was pooled and mixed with dialysate as described before (18).

In order to create a complete anaerobic environment, SLIM with $90 \mathrm{~mL}$ dialysate in each of the 4 individual units was flushed with gaseous nitrogen for at least 3 hours before incorporating the standardized microbiota. Thirty $\mathrm{mL}$ of the standardized microbiota was added to each SLIM-unit, making the total volume $120 \mathrm{~mL}$. Figure 1 shows the experimental set-up for fibre addition to SLIM. The microbiota was adapted to the model with SIEMP for 16 hours. During the adaptation phase, SIEMP was added into each SLIM-unit at a rate of $2.5 \mathrm{~mL} / \mathrm{h}$ through the feeding syringe. At the end of the adaptation period, a 2-hour starvation period was performed, which was used to allow all the carbohydrates within SIEMP to be fermented. Afterwards, a shot of 5 grams of the different RSMs was given to the system at time point 0 hours, and incubation was continued for $24 \mathrm{~h}$ after that (Figure 1).

\section{Sample collection}

Samples from lumen and spent dialysate were collected at time point $0,0.5,1,2,4,6,8$, and $24 \mathrm{~h}(\mathrm{t} 0, \mathrm{t} 0.5, \mathrm{t} 1, \mathrm{t} 2, \mathrm{t} 4, \mathrm{t} 6, \mathrm{t} 8$, and $\mathrm{t} 24)$. They were snap-frozen in liquid nitrogen and stored until analyses. Lumen samples were used to analyze microbiota composition and polysaccharides structures, and both lumen and dialysis samples were analyzed for short chain fatty acid (SCFA) concentrations.

\section{Sequencing of the V3-V4 region of the $16 \mathrm{~S}$ rRNA gene}

Microbial DNA extraction and sequencing of the V3-V4 region of the 16S rRNA gene were performed by BaseClear B.V. (Leiden, The Netherlands). Briefly, genomic DNA extraction was performed using the Quick-DNA ${ }^{\mathrm{TM}}$ Fecal/Soil Microbe Miniprep Kit (Zymo Research, California, USA) according to the manufacturer's instructions. Barcoded amplicons from the V3-V4 region of 16S rRNA genes were generated using a 2-step PCR. 10-25 ng genomic 
DNA was used as template for the first PCR with a total volume of $50 \mu \mathrm{L}$ using the $341 \mathrm{~F}\left(5^{\prime}\right.$ CCTACGGGNGGCWGCAG-3') and the 785R (5'-GACTACHVGGGTATCTAATCC-3') primers (22) appended with Illumina adaptor sequences. PCR products were purified (QIAquick PCR Purification Kit, Venlo, The Netherlands) and the size of the PCR products were checked on a Fragment analyzer (Advanced Analytical, Ankeny, US) and quantified by fluorometric analysis. Purified PCR products were used for the 2nd PCR in combination with sample-specific barcoded primers (Nextera XT index kit, Illumina, city, CA, USA). Subsequently, PCR products were purified, checked on a Fragment analyzer and quantified, followed by multiplexing, clustering, and sequencing on an Illumina MiSeq with the pairedend (2x) $300 \mathrm{bp}$ protocol and indexing. The sequencing run was analyzed with the Illumina CASAVA pipeline (v1.8.3) and demultiplexed based on sample-specific barcodes.

\section{Bioinformatics analysis}

The demultiplexed raw sequences obtained from BaseClear were processed using the QIIME2 pipeline (23). In short, reads were imported and quality filtered and dereplicated with q2-dada2 (24). Next, dada2 was performed with paired-end reads and truncations parameters were as follows: the first 17 and 14 base pairs were trimmed off in forward and reverse reads, respectively. And at position 280 base pairs the fragment was truncated in forward reads, and at position 230 base pairs for the reverse reads. The processed sequences were used for all the downstream analyses. Alpha-diversity (Shannon index) and $\beta$-diversity (weighted and unweighted UniFrac) were analyzed by the q2-phylogeny plugin (https://github.com/qiime2/q2-diversity).

Phylogenetic Investigation of Communities by Reconstruction of Unobserved States, PICRUSt2. The PICRUSt2 software (25) was used to predict microbial functional abundances based on marker gene sequences. KEGG database was used to predict the results.

\section{Chemical analyses}

Short-chain fatty acids analyses. Samples from lumen and dialysate were analyzed by Brightlabs (Venlo, The Netherlands) for determination of concentrations of SCFA. Ion exclusion chromatography (IEC) was applied on an 883 Ion Chromatograph (IC; Metrohm, Switzerland), using a Transgenomic IC Sep ICE-ION-300 column $(30 \mathrm{~cm}$ length, $7.8 \mathrm{~mm}$ diameter and $7 \mu \mathrm{m}$ particles) and a MetroSep RP2 Guard. The mobile phase consists of 1.5 $\mathrm{mM}$ aqueous sulphuric acid. Samples were centrifuged $(21,000 \mathrm{~g}, 10 \mathrm{~min})$ and the clear supernatant was filtered through a $0.45 \mu \mathrm{m}$ PFTE filter and diluted with mobile phase (for lumen 1:5, for dialysate 1:2). Ten microliters were loaded on the column by an autosampler 730 (Metrohm). Molecules were eluted according to their pKa.A column flow rate of 0.4 $\mathrm{ml}{ }^{*} \min ^{-1}$ was used. The temperature of the column was $65^{\circ} \mathrm{C}$. The organic acids were detected using suppressed conductivity detection

\section{Glycome profiling}

Sample preparation. Lumen samples from each time point and treatment were freeze-dried, after which they were dissolved at $1 \mathrm{mg} / \mathrm{mL}$ in deionized water, and stored at $-20^{\circ} \mathrm{C}$ as stock solutions.

Monoclonal antibodies ( $m A$ bs). $\mathrm{mAbs}$ were obtained as hybridoma cell culture supernatants from CarboSource (Atlanta, GA, USA) [http://www.carbosource.net/]. 
ELISA. The ELISA protocol was slightly modified from Pattathil et al. (2010) (26). In brief, samples prepared above were applied $(50 \mu \mathrm{L}$ of $100 \mu \mathrm{g} / \mathrm{mL}$ in deionized water per well) to Costar 3598 96-well plates (Corning Life Sciences, city, country) and were dried to the well surfaces by evaporation overnight at $37^{\circ} \mathrm{C}$. Control wells contained deionized water. The plates were blocked with $200 \mu \mathrm{l}$ of $1 \%$ (w/v) bovine serum albumin (BSA) in Tris-buffered saline (50 mM Tris- $\mathrm{HCl}$, pH 7.6, containing $100 \mathrm{mM}$ sodium chloride) for $1 \mathrm{~h}$. Blocking agent was removed by aspiration, and $50 \mu \mathrm{l}$ of undiluted hybridoma supernatant were added to each well and incubated for $1 \mathrm{~h}$ at room temperature. Supernatant was removed and wells were washed three times with $200 \mu \mathrm{l}$ of $0.1 \%$ (w/v) BSA in Tris-buffered saline (wash buffer). Peroxidase-conjugated goat anti-mouse IgG, anti-mouse IgM, goat anti-rat IgG, or goat antirat IgM antibodies (Sigma-Aldrich), depending on the primary antibody used, were diluted 1:5,000 in wash buffer, and $50 \mu \mathrm{l}$ were added to each well and incubated for $1 \mathrm{~h}$. Wells were then washed five times with $200 \mu \mathrm{l}$ of wash buffer. Next, 3,3',5,5'-tetramethylbenzidine (TMB) solution (Sigma-Aldrich, St. Louis, USA) was freshly prepared according to the manufacturer's instructions, and $50 \mu \mathrm{L}$ were added to each well. After 20 min, the reaction was stopped by adding $50 \mu \mathrm{l}$ of $0.5 \mathrm{~N}$ sulfuric acid to each well. The OD of each well was read at a wavelength of $450 \mathrm{~nm}$ using a Multi-mode microplate reader (BioTek Synergy HTX, Abcoude, The Netherlands).

Polysaccharide panel screening. Polysaccharide panel screening of mAbs was carried out by ELISA against all lumen samples immobilized to $96-w e l l$ plates. Duplicate preparations of each polysaccharide were used for all experiments reported here. Binding data was visualized in $\mathrm{R}$ via ComplexHeatmap package (27).

\section{Statistics}

Kruskal-Wallis Rank Sum Test (one-way ANOVA on ranks) was applied to compare $\alpha$ diversities (Shannon index) among different RSM treatments and time points, and Wilcoxon Rank Sum Test was used for pairwise comparison in R version 3.5.3 (https://www.rproject.org/). Bonferroni was used to correct $P$-values. Permutational multivariate analysis of variance (PERMANOVA; REFERENCE) was performed to test the significance of $\beta$ diversity distances in QIIME2 (weighted and unweighted UniFrac) between non-processed and processed RSM. The results were visualized in $\mathrm{R}$ ( $\mathrm{R}$ version 3.5.3).

The amplicon sequence variant (ASV) table (feature table of QIIME2) was normalized and filtered in R, and statistical analysis and visualizations were performed in R and STAMP (Parks, Tyson et al. 2014). The table was normalized via division by the sum of sequences in a given sample and multiplied by the minimum sum across all samples. Relative abundances were filtered as follows: values below a relative abundance threshold of $0.01 \%$ were not taken into account; taxa with a median relative abundance $<1 \%$ in all groups were not considered for statistical analysis. White's non-parametric t-test was applied to compare between CON and treatments. $P$-values were corrected using the Benjamini-Hochberg method. A $q$-value (corrected $P$-value) $<0.05$ was considered significant.

Pearson correlations between continuous meta-variables and taxonomic variables were calculated and visualized in R. Parameters were set as follows: Missing values for metavariables were handled as NO imputation (replacing missing data with substituted); zeros were kept for the calculation of correlation; a minimum number of $0.1 \%$ was considered for calculation; a minimum of 4 paired observations were required for calculation of correlations. 
$P$-values were corrected using the Benjamini-Hochberg method. A q-value (corrected Pvalue $)<0.05$ was considered significant.

SCFA production between CON and the treated RSM substrates were compared and visualized in $\mathrm{R}$.

\section{Results}

\section{Characteristics of non-processed and processed RSM}

A comprehensive set of 155 plant cell wall glycan-directed monoclonal antibodies was used to screen untreated RSM by a ELISA-based assay $(26,28)$, and 34 antibodies reacted with RSM (data not shown). These were subsequently used in the current study to obtain information on the presence and relative abundance of specific epitopes that are characteristic of the different types of polymers in untreated RSM and RSM processed by ALK, CELL, PECT1, and PECT2.

Figure 2 shows that both increases and decreases in epitope recognition occurred in ALK, CELL, PECT1, and PECT2 compared to CON. Samples from after ( A) and before (_B) predigestion clustered together according to each treatment, which indicated_A and B B from the same treatment had similar epitope accessibility. ALK strongly increased binding of nonfucosylated XG mAbs, while CELL, PECT1, and PECT2 led to disappearance of those compared to CON, regardless of _A and _B treatment. All the treatments increased the binding of "Linseed Mucilage RG-I group" directed mAbs, but had little effect on Xylan-2 and RG-Ic group compared to CON. Binding of MAC204 (AG-1), which is binding to gum tragacanth and to lettuce and green tomato RG-I preparations (arabinogalactan), disappeared with ALK_A, ALK_B, and CELL_B, while increased binding of CCRC-M107 (AG-2), which binds to linear and branched arabinans and RG-I preparations from diverse plants but does not bind to larch arabinogalactan (26), was observed in ALK_A and ALK_B. CELL_A, CELL_B, PECT1_A, PECT1_B, and PECT2_B led to disappearance of the binding of mAbs of "pectic backbone group" and CCRC-M 133, which also binds to linear and branched arabinans and RG-I preparations from diverse plants but do not bind to larch arabinogalactan. ALK, PECT1, and PECT2 increased binding of $m A b s$ directed against the arabinogalactan side chains of RG-I (RG-I/AG). 

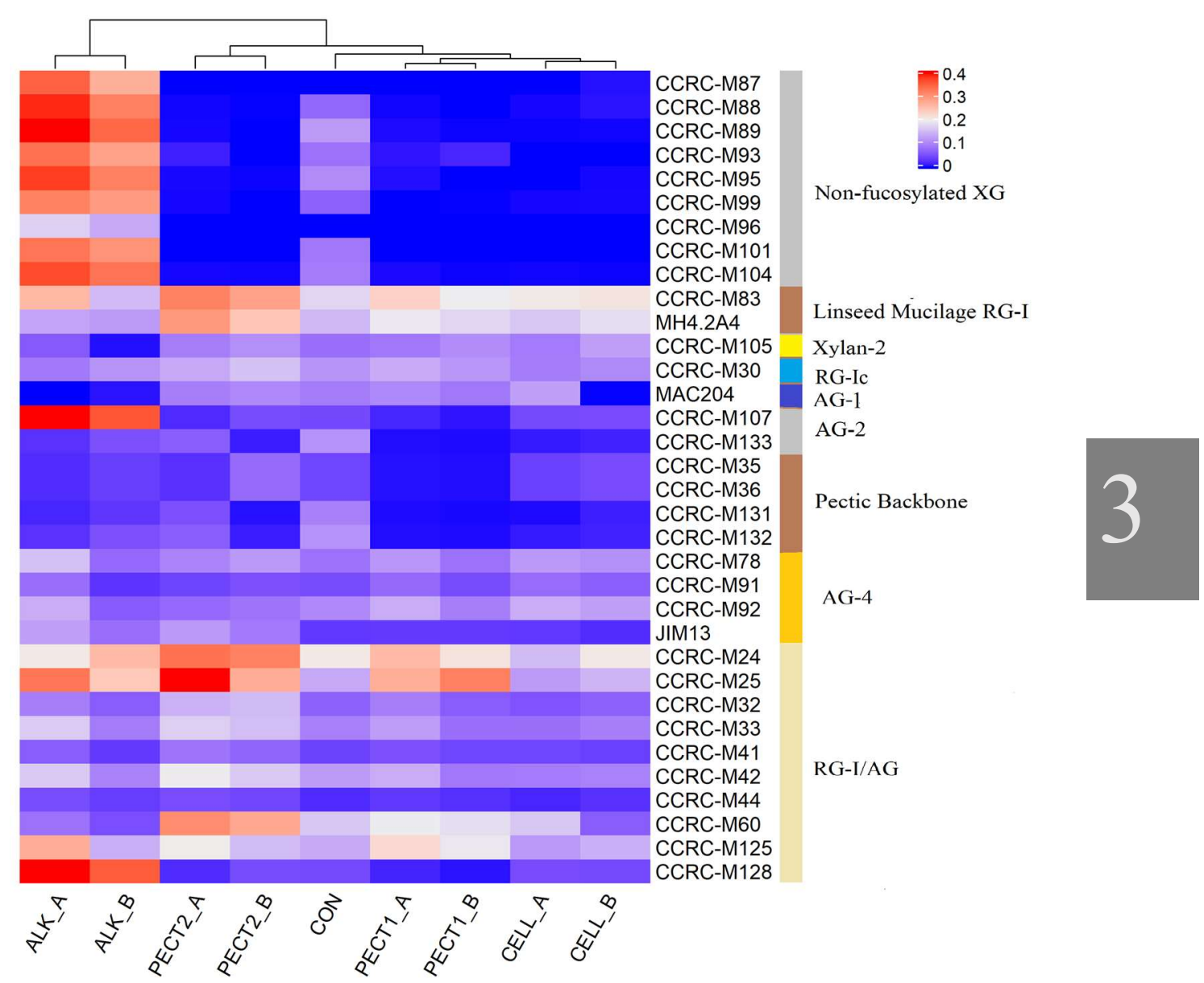

Figure 2. Glycome profiling of non-processed and processed RSM. The binding response data of the mAbs are presented as heatmap using a blue-white-red scale indicating the intensity of the ELISA signal (blue, white, and red colors depict no, medium, and strong binding, respectively). The mAbs (indicated by their codes) are grouped based on the cell wall glycans they predominantly recognize as shown in the panel on right-hand side of the figure._A, RSM was treated after predigestion,_B; RSM was treated before predigestion. XG, xyloglucan; RG-I, rhamnogalacturonan I; AG, arabinogalactan; RG-I/AG, arabinogalactan side chains of RG-I.

\section{ALK, CELL, PECT1, and PECT2 significantly changed microbiota composition compared to $\mathrm{CON}$}

To determine the changes in composition of the gut microbiota fed with a shot of $5 \mathrm{~g} \mathrm{CON}$, ALK, CELL, PECT1, or PECT2, a comparison of microbiota based on sequencing the V3$\mathrm{V} 4$ region of the $16 \mathrm{~S}$ rRNA gene was performed. Shannon indeces significantly decreased at $\mathrm{t} 4, \mathrm{t} 6, \mathrm{t} 8$ and $\mathrm{t} 24$, compared to that of $\mathrm{t} 0$ (Figure S1A). When data from all of the time points were pooled, there were no significant differences among CON, ALK, CELL, PECT1, and 
PECT2 in Shannon index (Figure S1B). Phylogeny based UniFrac methodology was then used to compare the $\beta$-diversity of the microbial communities between microbiota fed with non-processed and processed RSM. Unweighted UniFrac analysis (Figure 3) shows that samples from processed RSM (ALK, CELL, PECT1, and PECT2) significantly $(P=0.004)$ separated from non-processed RSM (CON), and samples from different processed method clustered together. Samples from CON, ALK, CELL, PECT1, and PECT2 all clustered together $(P=0.125)$ with respect to weighted UniFrac (Figure $\mathrm{S} 2$ ).

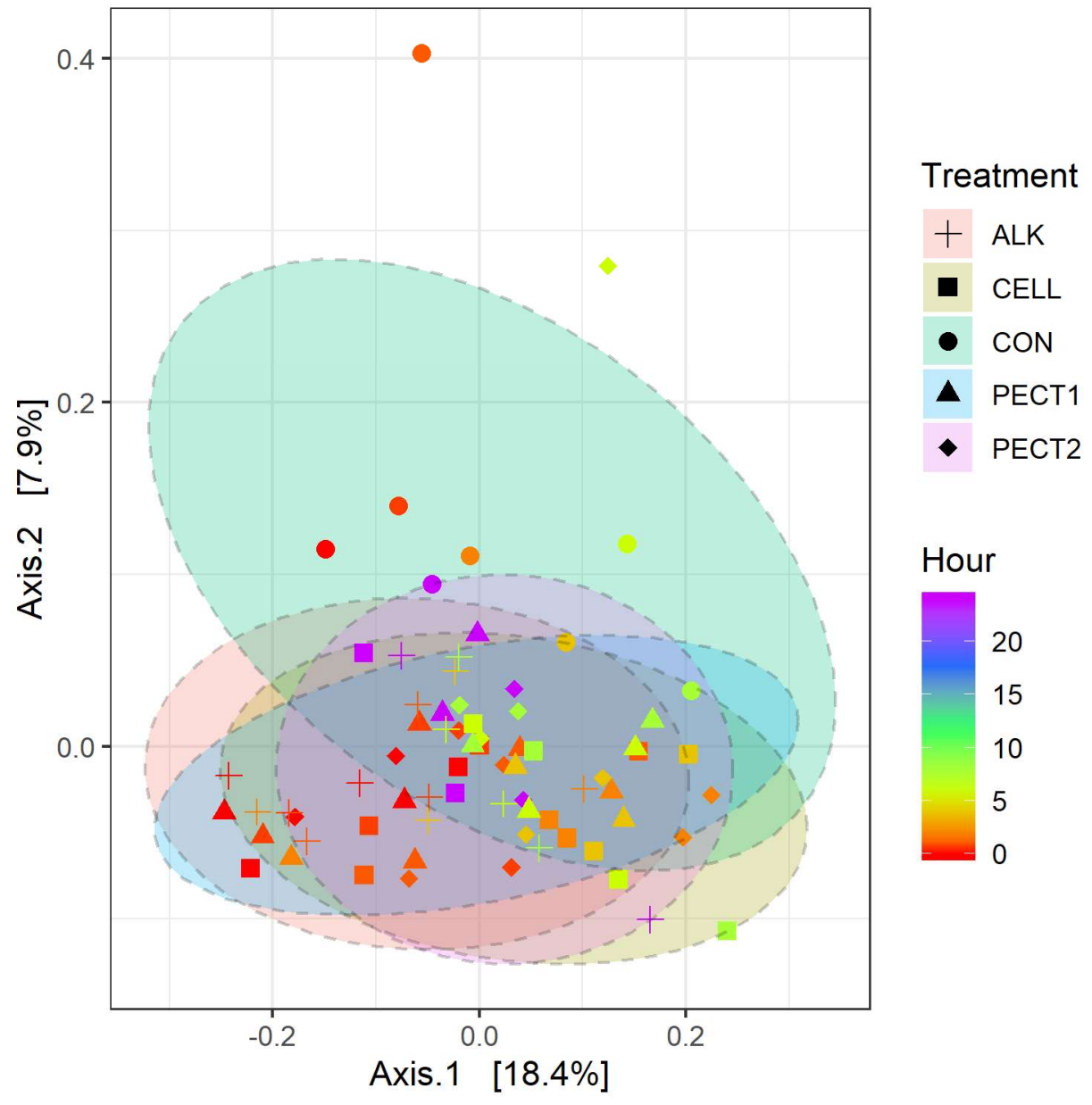

Figure 3. Principal coordinate analysis (PCoA) plot generated based on the calculated distances in unweighted UniFrac matrix. Samples were grouped by shape and color in terms of treatment and time point they belonged to, respectively: CON (sphere), ALK (plus), PECT1 (triangle), PECT2 (diamond), CELL (square); a red-green-purple scale was used to indicate the fermentation time (red and purple depict start and end of the fermentation period).

There were no significant differences between microbiota fed with RSM predigested before 
or after carbohydrase or ALK treatment with respect to both $\alpha$-diversity (Figure S3), or $\beta$ diversity (data not shown), which indicated predigesting before or after processing RSM had little effect on microbiota composition. Glycome profiling of RSM (Figure 2) also shows that samples from after and before processing clustered together according to each treatment, which indicated their polysaccharide compositions were similar to each other. Therefore, they were treated as duplicates in the following (microbial relative abundance) analyses.

Relative abundances of taxa within the pig microbiotas fed with non-processed and processed RSM were compared to identify significantly different bacterial taxa. At genus level (Figure 4), seven genera were significant higher in relative abundance after ALK, CELL, PECT1, and PECT2 treatment compared to CON. These were Ruminococcaceae NK4A214 group, Ruminococcaceae UCG-002, Ruminococcaceae UCG-005, Roseburia, Anaerotruncus, Bifidobacterium, Christensenellaceae $R-7$ group, and Selenomonas. For genera Christensenellaceae $R-7$ group and Ruminococcaceae UCG-005, their relative abundances were also higher in ALK and PECT1 compared to CELL. Instead, the relative abundances of Prevotella 7, an unclassified genus from Prevotellaceae, and Prevotellaceae UCG-001 were significantly decreased after feeding ALK, CELL, PECT1, and PECT2 compared to microbiota fed with CON. The relative abundance of Succinivibrionaceae UCG-001 was significant higher in ALK and PECT1 compared to CON.
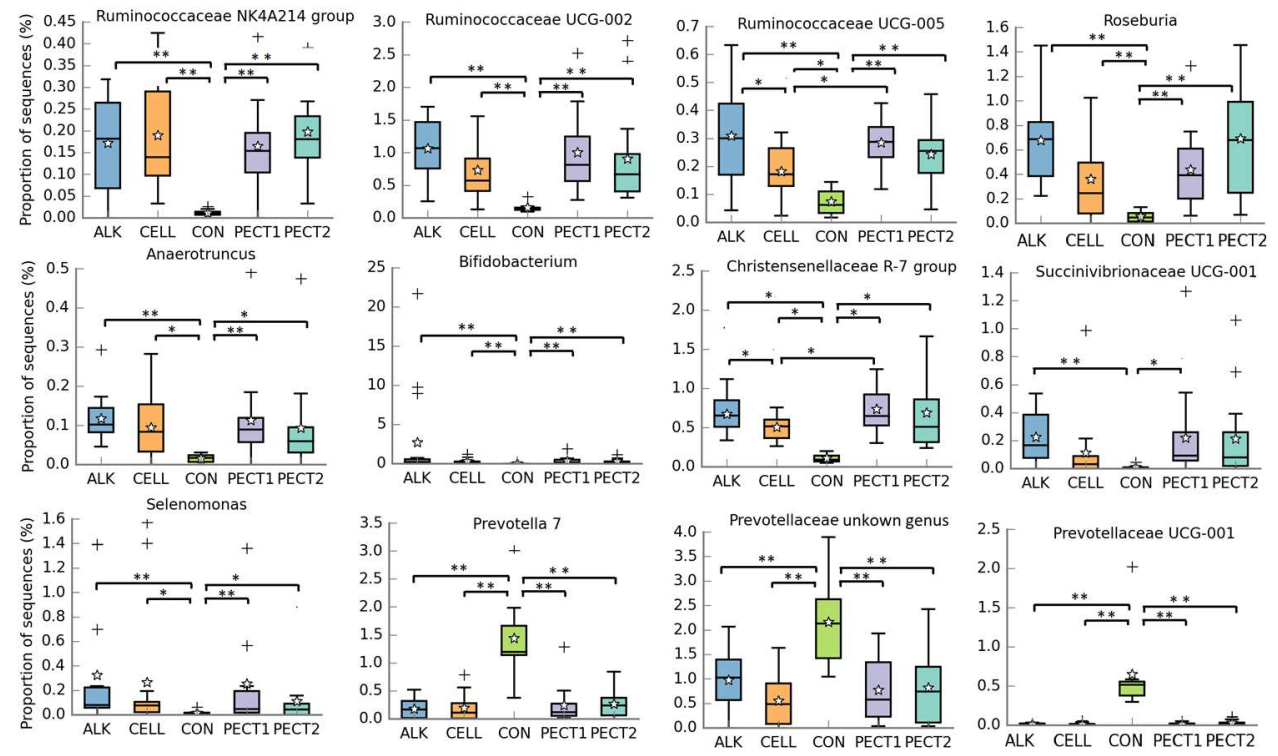

Figure 4. Significantly different relative abundances of microbial genera upon treatment with ALK, CELL, CON, PECT1, and PECT2. * $\mathrm{P}<0.05 ; * * \mathrm{P}<0.01$

PICRUSt2 analyses revealed that microbial functional abundances related to carbohydrate metabolism and SCFA production were significantly increased with processed RSM compared to CON

PICRUSt2 was performed to the $16 \mathrm{~S}$ rRNA gene data to predict metagenomic functional profiles. In this study we focused on carbohydrate metabolism related microbial functions 
(Figure 5). The relative abundances of fibre degradation pathways, beta-glucosidase [EC:3.2.1.21], beta-mannosidase [EC:3.2.1.25], cellobiose phosphorylase [EC:2.4.1.20], and sucrose phosphorylase [EC:2.4.1.7], were significant higher in ALK, CELL, PECT1, and PECT2 compared to CON, whereas conversely that of alpha-L-fucosidase [EC:3.2.1.51] was significant higher in CON compared to ALK, CELL, PECT1, and PECT2. For cellobiose phosphorylase [EC:2.4.1.20] and sucrose phosphorylase [EC:2.4.1.7], the relative abundances in ALK were also significant higher than those of CELL, PECT1, and PECT2.

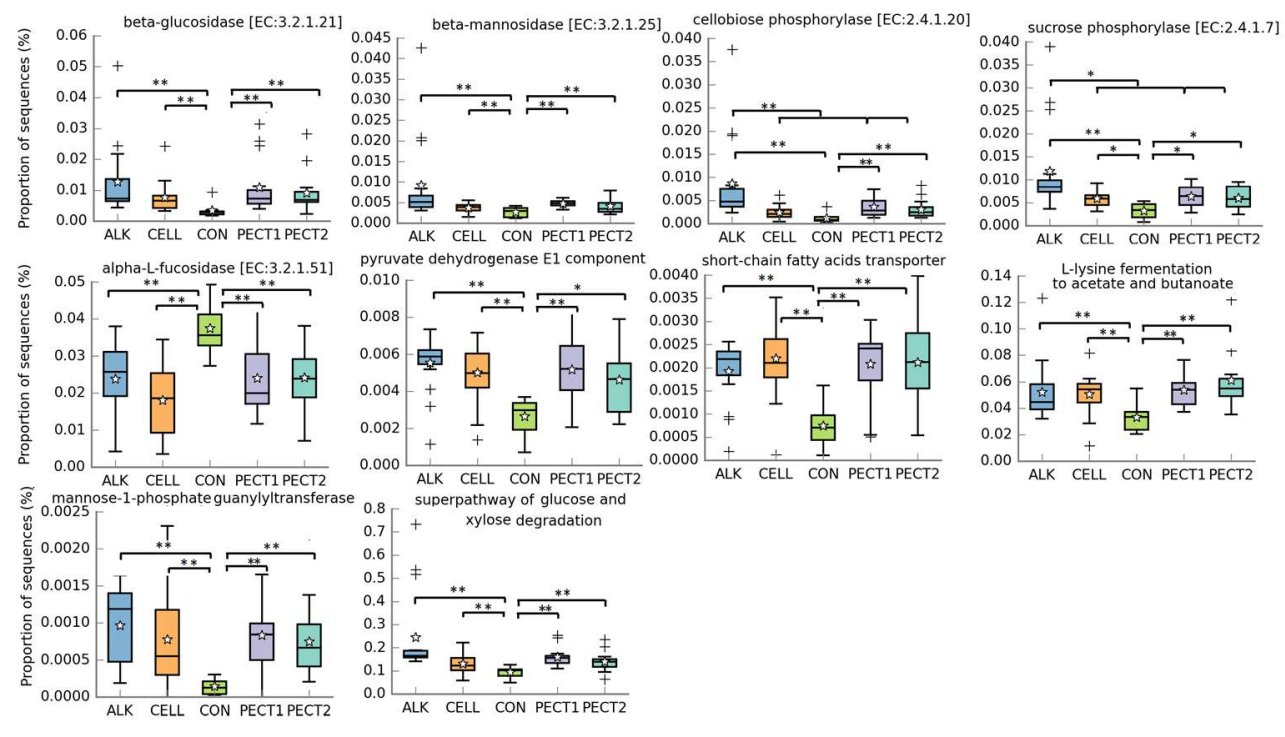

Figure 5. Significantly different metagenomic functions in relative abundance among ALK, CELL, CON, PECT1, and PECT2. * $\mathrm{P}<0.05 ; * * \mathrm{P}<0.01$.

Six microbial pathways related to fermentation were significant higher in relative abundance when microbiotas were fed with ALK, CELL, PECT1, and PECT2 compared to microbiota fed with CON. These pathways were pyruvate dehydrogenase E1 component, short-chain fatty acids transporter, mannose-1-phosphate guanylytransferase, superpathway of glucose and xylose degradation, sucrose degradation IV, and L-lysine fermentation to acetate and butanoate. The relative abundance of lactose/L-arabinose transport system permease protein was significant higher in ALK, PECT1 and PECT2 compared to CON.

Figure 6 shows that the cumulative acetic, propionic, and butyric acid and total SCFA production were higher in ALK, CELL, PECT1, and PECT2 compared to CON. For acetic acid, more than 2 times greater production was observed when the microbiota was fed with ALK, CELL, PECT1, and PECT2 compared to when the microbiota was fed with CON. The production of propionic, butyric acid and total SCFA in ALK, CELL, PECT1, and PECT2 were more than 1.6 times higher than that in $\mathrm{CON}$, except for propionic (1.3 times), and butyric acid (1.4 times) production in ALK. 

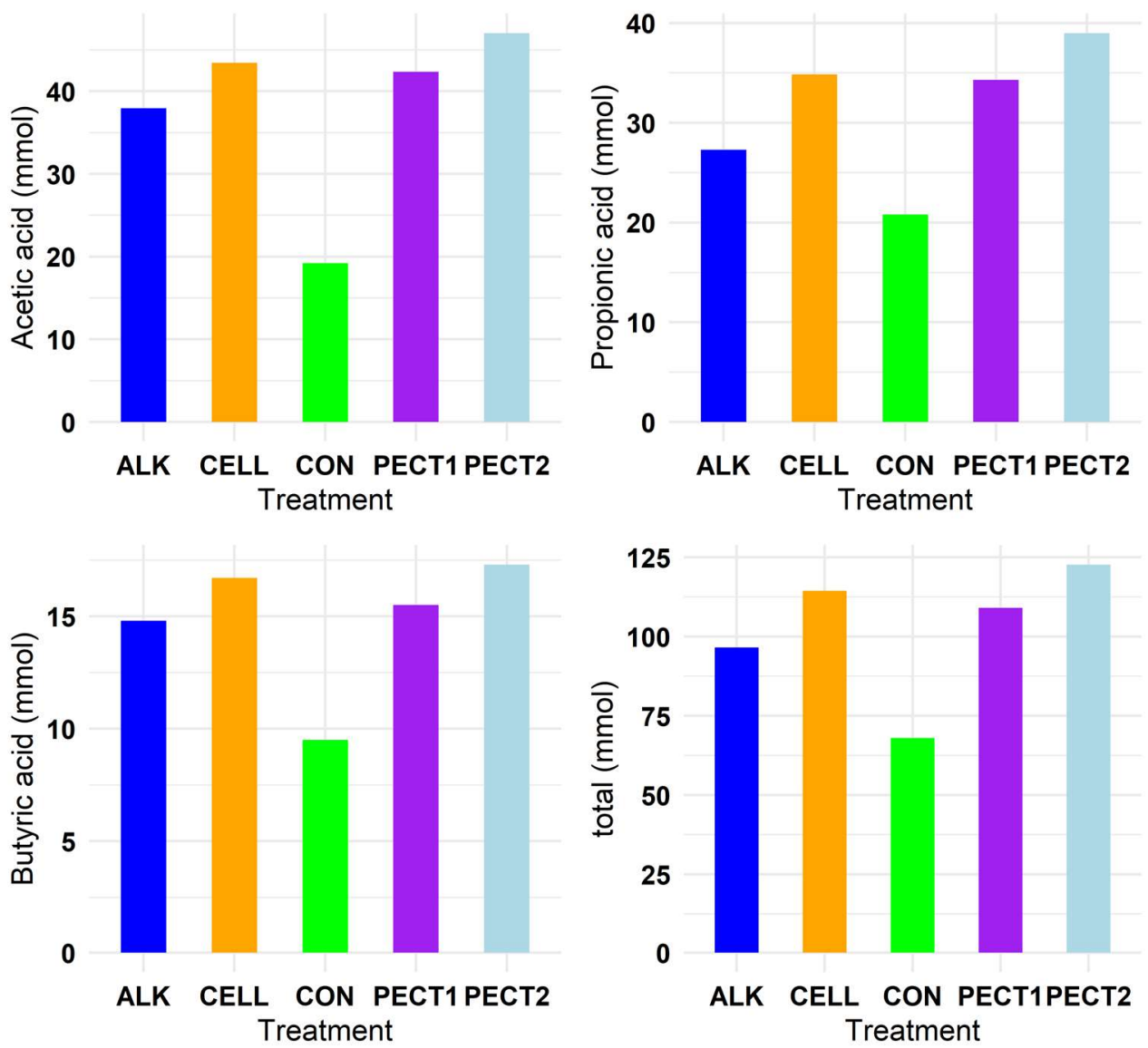

\begin{tabular}{|c|c|c|c|c|}
\hline Treatment & acetic.acid & propionic.acid & butyric.acid & total \\
\hline ALK & 2.0 & 1.3 & 1.6 & 1.4 \\
\hline CELL & 2.3 & 1.7 & 1.8 & 1.7 \\
\hline PECT1 & 2.2 & 1.6 & 1.6 & 1.6 \\
\hline PECT2 & 2.5 & 1.9 & 1.8 & 1.8 \\
\hline
\end{tabular}

Figure 6. Cumulative Acetic (A), Propionic (B), and Butyric acid (C), and total short-chain fatty acid (D) production during fermentation of ALK, PECT1, PECT2, and CELL compared to CON. Fold change of short-chain fatty acid in ALK, PECT1, PECT2, and CELL compared to $\mathrm{CON}(\mathrm{E})$.

Glycome profiling shows that binding of mAbs in lumen digests were dynamic during the in vitro fermentation in SLIM 
To investigate the dynamic changes of polysaccharides structure in CON, ALK, CELL, PECT1, and PECT2 during in vitro fermentation, a time series of sampling was performed and the set of $34 \mathrm{mAbs}$ was used to screen the lumen digests. Figure 7 shows that no or few binding signals were observed in Non-fucosylated XG, AG-2, and Pectic Backbone mAbs upon feeding CON, ALK, CELL, PECT1, and PECT2 at all time points.

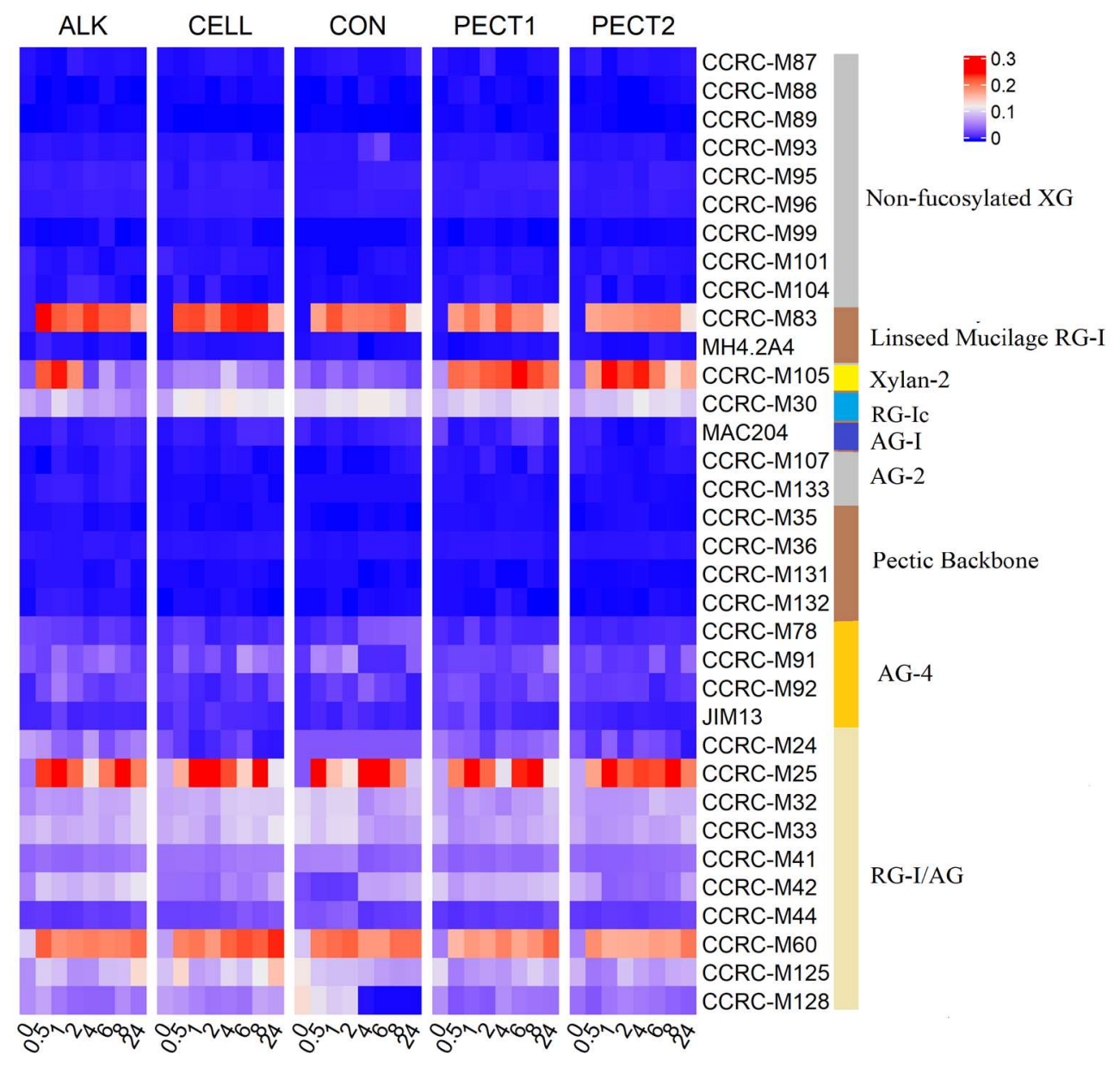

Figure 7. Glycome profiling of lumen digests during in vitro fermentation of ALK, CELL, CON, PECT1, and PECT2 at time point $0,0.5,1,2,4,6,8$, and $24 \mathrm{~h}$. The binding response data are presented as heatmap using a blue-white-red scale indicating the strength of the ELISA signal (blue, white, and red colors depict no, medium, and strong binding, respectively). The mAbs are grouped based on the cell wall glycans they predominantly recognize as shown in the panel on the right-hand side of the figure. XG, xyloglucan; RG-I, rhamnogalacturonan I; AG, arabinogalactan; RG-I/AG, arabinogalactan side chains of RG-I.

No binding of CCRC-M83 that specifically bind to Linseed Mucilage RG-I was observed at t0 in all treatments (just prior to addition of the fibre shots), and binding signals appeared 
afterwards. For ALK, binding of CCRC-M83 decreased from t0.5 to t2, increased again at $\mathrm{t} 4$, and decreased to the lowest value at $\mathrm{t} 24$. For CELL, binding of CCRC-M83 decreased from t0.5 to t2, stabilized from $t 4$ to $t 8$, and decreased to the lowest value at t 24 . For CON, binding increased from $t 0.5$ to $t 1$, decreased from $t 2$ to $t 6$, increased at $t 8$, and decreased to the lowest value at $\mathrm{t} 24$. For PECT1, binding increased from $\mathrm{t} 0.5$ to $\mathrm{t} 4$, and then decreased progressively until t24. For PECT2, binding increased slightly from t0.5 to $t 8$, and then decreased to the lowest value at $\mathrm{t} 24$.

In terms of Xylan-2 recognizing $\mathrm{mAb}$ (i.e. CCRC-M105), weak bindings were detected at all time points after t0 with CELL and CON. Increased binding of CCRC-M105 was observed from t0.5 to $t 1$ in ALK and the binding strength decreased from $t 2$ to $t 4$, slightly increased again at t6, and then decreased until t24. For PECT1, increased binding of CCRC-M105 was observed from t0.5 to t6, which decreased afterward until t24. Binding for PECT2 was dynamic from t0.5 to 24 , but the lowest binding was observed at $\mathrm{t} 24$.

As for the RG-Ic recognizing mAb (i.e. CCRC-M30), binding of CCRC-M30 was lower in ALK, PECT1, and PECT2 according to each time point compared to CELL and CON, but binding over time was dynamic. With respect to the AG-4 mAbs (recognizing arabinogalactans), weak and dynamic binding was observed at each time point in all treatments.

With respect to RG-I/AG mAbs, more active mAbs were observed compared to other groups of mAbs in all treatments. Within RG-I/AG mAbs, binding strengths of CCRC-M25 and CCRC-M60 were stronger than other RG-I/AG mAbs in all treatments, and their binding strengths were fluctuating during the whole fermentation period and still existed at 24 in all treatments.

\section{Correlation between microbiota abundance and SCFA production and mAb binding}

Pearson correlation analyses were performed to investigate the relationship between the relative abundance of microbial genera and SCFA production at each time point (Figure 8). Seven genera (Bifidobacterium, Collinsella, Denitrobacterium, Olsenella, Coriobacteriaceae.1, Bacteroidales S24-7 group.2, and Acetitomaculum) had significant negative correlation with propionic acid, butyric acid, valeric acid and total SCFA production. Within these, Bacteroidales S24-7 group.2, Olsenella, Coriobacteriaceae.1, and Acetitomaculum also significantly negatively correlated with acetic acid. Eight genera (Bacteroidales S24-7 group.1, Prevotella 9, Faecalibacterium, Ruminococcaceae UCG-005, Ruminococcus 2, Selenomonas, Succinivibrio, and Succinivibrionaceae UCG-001) significantly positively correlated with acetic acid, propionic acid, butyric acid, valeric acid and total SCFA production. Within these, Ruminococcus 2 and Succinivibrio also had significant positive correlation with caproic acid. Bacteroidales S24-7 group, Sarcina, and Oribacterium had significant positive correlation with propionic acid, butyric acid, valeric acid and total SCFA production. Roseburia, Ruminococcaceae NK4A214 group and Ruminococcaceae UCG-002 significantly positively correlated with acetic acid, propionic acid, butyric acid and total SCFA production. Prevotella 7 significantly positively correlated with propionic acid, valeric acid, caproic acid, and total SCFA production. 


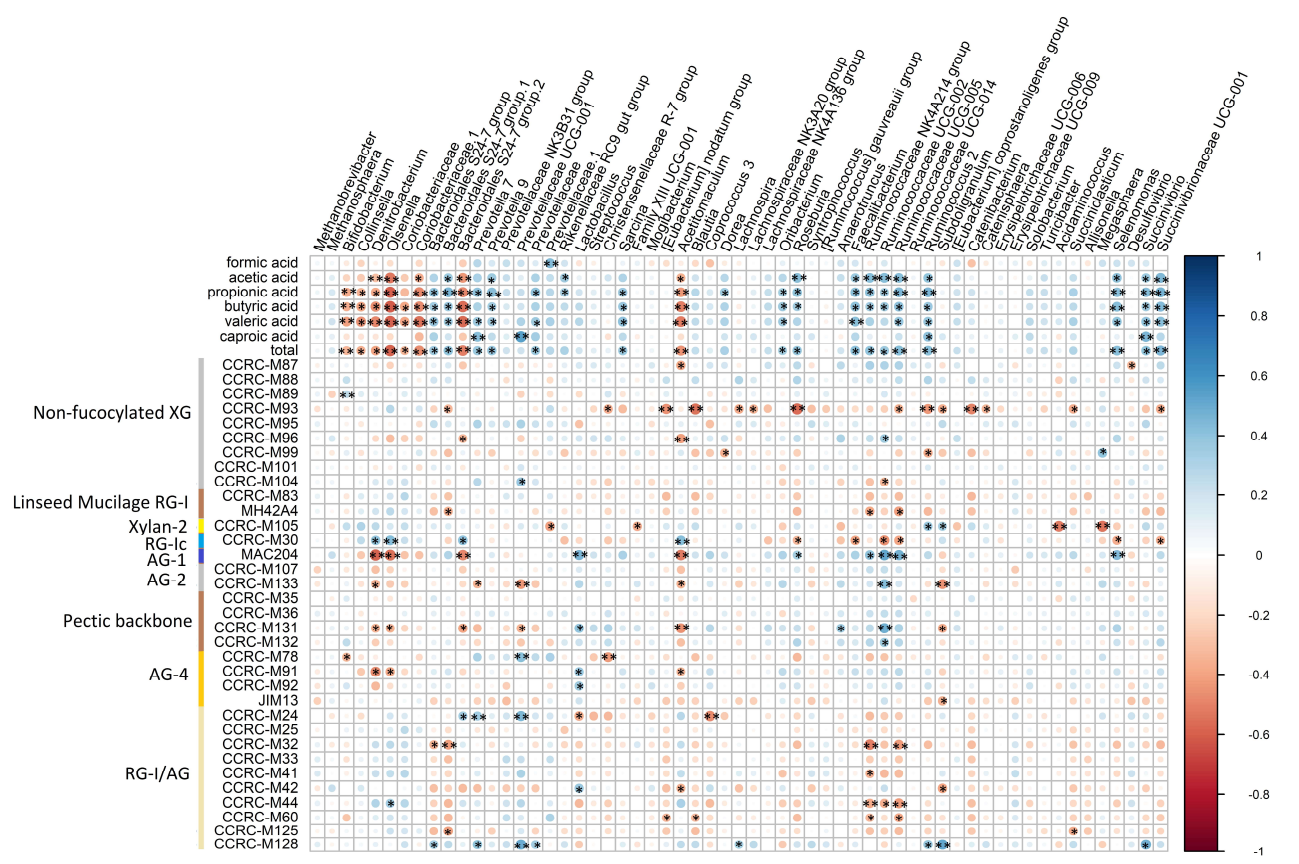

Figure 8: Correlation between core bacterial genera, and SCFA and binding of mAbs. Statistical significance was determined for all pairwise comparisons using Pearson's method. $* \mathrm{P}<0.05$; ** $\mathrm{P}<0.01$. Size of the circles indicates correlation strength, which bigger sizes meaning higher correlations. Blue circles represent positive correlations (correlation coefficients from 0 to 1 ), whereas red circles represent negative correlations (correlation coefficients from 0 to -1). total, total SCFA production. The mAbs are grouped based on the cell wall glycans they predominantly recognize as shown in the panel on the left-hand side of the figure. XG, xyloglucan; RG-I, rhamnogalacturonan I; AG, arabinogalactan; RG-I/AG, arabinogalactan side chains of RG-I.

The correlation between binding of mAbs and relative abundance of microbial genera was also analyzed. Within the mAbs that recognizing non-fucocylated XG, CCRC-M93 had significant negative correlation with Bacteroidales S24-7 group.1, Christensenellaceae R-7 group, [Eubacterium] nodatum group, Blautia, Lachnospira, Lachnospiraceae NK3A20 group, Roseburia, Ruminococcaceae UCG-005, Ruminococcus 2, Subdoligranulum, Catenibacterium, Catenisphaera, Succiniclasticum, and Succinivibrionaceae UCG-001. CCRC-M96 significantly negatively correlated with Bacteroidales S24-7 group.2 and Acetitomaculum, while it significantly positively correlated with Anaerotruncus. CCRCM99 had significant negative correlation with Dorea and Ruminococcus 2, whereas it had significant positive correlation with Megasphaera. CCRC-M104 had significant positive correlation with Prevotellaceae UCG-001, while it negatively correlated with Ruminococcaceae UCG-002.

MH4.2A4 that recognizes linseed mucilage RG-I had significant negative correlations with Bacteroidales S24-7 group.1, Ruminococcaceae NK4A214 group, and Ruminococcaceae UCG-005. 
CCRC-M105 that binds to Xylan-2 had significant negative correlations with Prevotellaceae.1, Family XIII UCG-001, Acidaminococcus, and Megasphaera, whereas it positively correlated with Ruminococcus 2 and Subdoligranulum.

CCRC-M30, binding to RG-Ic, had significant positive correlations with Denitrobacterium, Olsenella, Bacteroidales S24-7 group.2, and Acetitomaculum, while it significantly negatively correlated with Roseburia, Faecalibacterium, Ruminococcaceae UCG-002, Ruminococcaceae UCG-005, Selenomonas, and Succinivibrionaceae UCG-001.

MAC204, that recognizing AG-1, significantly negatively correlated with Denitrobacterium, Olsenella, Bacteroidales S24-7 group.2, Acetitomaculum, and Roseburia, while it had significant positive correlations with Lactobacillus, Sarcina, Roseburia, Ruminococcaceae NK4A214 group, Ruminococcaceae UCG-002, Ruminococcaceae UCG-005, and Selenomonas.

CCRC-M133, binding to AG-2, significantly negatively correlated with Denitrobacterium, Prevotella 7, Prevotellaceae UCG-001, Acetitomaculum, and Subdoligranulum, while it positively correlated with Ruminococcaceae UCG-002.

CCRC-M131, that binds to pectic backbone, had significant negative correlation with Denitrobacterium, Olsenella, Bacteroidales S24-7 group.2, Prevotellaceae UCG-001, Acetitomaculum, and Subdoligranulum, while it positively correlated with Lactobacillus, Anaerotruncus, and Ruminococcaceae UCG-002.

With respect to mAbs binding to AG-4, CCRC-M78 had significant negative correlation with Bifidobacterium, and Christensenellaceae $R-7$ group, whereas it significantly positively correlated with Prevotellaceae UCG-001. CCRC-M91 had significant negative correlation with Denitrobacterium, Olsenella, and Acetitomaculum, and CCRC-M91 and CCRC-M92 significantly positively correlated with Lactobacillus. JIM13 had significant negative correlation with Subdoligranulum.

As for mAbs binding to RG-I/AG, they had significant negative correlation with Bacteroidales S24-7 group (CCRC-M32), Bacteroidales S24-7 group.2 (CCRC-M32 and M125), Lactobacillus (CCRC-M24), [Eubacterium] nodatum group (CCRC-M60), Acetitomaculum (CCRC-M42), Blautia (CCRC-M60), Coprococcus 3 (CCRC-M24), Ruminococcaceae NK4A214 group (CCRC-M32, M41, M44, and M60), Ruminococcaceae UCG-002 (CCRC-M44), Ruminococcaceae UCG-005 (CCRC-M32, M44, and M60) Subdoligranulum (CCRC-M 42), and Succiniclasticum (CCRC-M125). Significantly positively correlations were observed with Olsenella (CCRC-M44), Bacteroidales S24-7 group (CCRC-M128), Prevotella 7 (CCRC-M24 and M128), Prevotellaceae UCG-001 (CCRC-M 24 and M128), Prevotellaceae (CCRC-M128), Lactobacillus (CCRC-M42), Lachnospira (CCRC-M128), Ruminococcus 2 (CCRC-M128), Subdoligranulum (CCRCM128), and Succinivibrio (CCRC-M128).

\section{Discussion}

\section{Cell wall polysaccharides composition of differently pretreated RSM}

Cell wall polysaccharides of RSM (Brassica napus) were comprehensively studied by chemical methods (29), which showed that they generally consisted of arabinan, 
homogalacturonan, Rhamnogalacturoan I (RG-I), type II arabinogalactan (AG), and xyloglucan (XG) (30). In a previous study (Long, de Vries and Venema submitted), we determined the monosaccharide constituent composition, which was in line with Pustjens et al. (2013). Our current findings with the $\mathrm{mAbs}$ are also in line with this, since nonfucosylated XG-, RG-I-, pectic backbone-, and AG-recognizing mAbs bound to CON. By similar reasoning, xylan was also detected in $\mathrm{CON}$, which was not reported before, although it is not entirely clear how much cross-reactivity the mAbs show.

ALK treatment intensively increased binding of mAbs that specifically bind to nonfucosylated XG (Figure 2). This is consistent with previous finding that alkali could extract XG from hemicellulose (29), which led to XG being detected by the mAbs in the current study. Previous research also demonstrated that XG is linked via hydrogen bridges to the surface of cellulose microfibrils, which is extractable with alkali, but not accessible by enzyme (31). However, enzymatic treatments (PECT1, PECT2, and CELL) led to a disappearance of binding nonfucosylated XG-specific mAbs compared to CON, which was unexpected. It could be that XG might become entrapped by other cell wall structures after pectinase or cellulase treatment. It is unknown whether this kind of the hidden phenomenon would reduce the degradability of XG.

Enzymatic treatments increased binding of linseed mucilage RG-I-specific mAbs and some mAbs directed against the arabinogalactan side chains of RG-I (RG-I/AG), and reduced binding of some mAbs directed to pectic backbone, which indicated that PECT1 and PECT2 broke down pectic backbone and exposed RG-I and its arabinogalactan side chains. These results are supported by a previous study showing that PECT1 and PECT2 degraded pectic backbone and released RG-I and its arabinogalactan side chains (29). Moreover, RG-I attached to cellulose microfibrils can be released by cellulase (32), while at the same time it might block the accessibility of pectic backbone because of the shifting of the polysaccharides structure.

ALK treatment led to the disappearance of binding of mAbs that specifically bind to AG-1 while it increased binding of mAbs that specifically binding to AG-2, which indicated that ALK could cause arabinogalactan to be physically entrapped in other cell wall structures, while linear and branched arabinan become accessible (26).

They were lower in binding strengths of mAbs in AG-4 from ALK_B, PECT1_B, PECT2_B, and CELL_B, compared to ALK_A, PECT1_A, PECT2_A, and CELL_A, respectively. This indicated that small fragments were produced in AG-4 group after enzymatic and chemical treating RSM, which were dialyzed out in ALK_B, PECT1_B, PECT2_B, and CELL_B during the subsequent predigestion treatment. Overall, both enzymatic and chemical processed RSM can release some polysaccharides but physically entrap or shield some others at the same time.

\section{Community structure of swine microbiota fed with differently pretreated RSM}

The PCoA analysis showed significant difference of both enzymatic and chemical treatments on swine microbial community $(P=0.004)$ compared to $\mathrm{CON}$, based on unweighted UniFrac metric (Figure 3); and overall there were no significant differences in this metric between treatments (Figure 3). Dietary fibre is known to have a considerable effect on gut microbiota composition (33-35). Glycome profiling of non-processed and processed RSM showed that 
polysaccharides structures were differentially shifted due to ALK, PECT1, PECT2, and CELL treatment in the current study, which were consistent with previous studies $(10,12)$. These observations might explain the specific genus changes in microbiota composition after microbiotas fed with ALK, PECT1, PECT2, and CELL compare to CON (Figure 4), despite the differential effect of the different treatments as assessed by the glycome profiling.

In order to understand which microbes were significantly influenced by dietary supplementation of processed RSM, microbial genera were compared among the five treatments (Figure 4). The relative abundances of Ruminococcaceae NK4A214 group, Ruminococcaceae UCG-002, Ruminococcaceae UCG-005, Roseburia, Bifidobacterium, and Christensenellaceae $R-7$ group were significantly increased in ALK, PECT1, PECT2, and CELL (Figure 4). Research has shown that genera of family Ruminococcaceae contain major (hemi)cellulolytic and pectinolytic species (36-38). Thus, microbes from family Ruminococcaceae play an important role in degrading (hemi)cellulose and pectin in their activity against recalcitrant fibre $(39,40)$. Previous research demonstrated that the higher the relative abundance of Ruminococcaceae NK4A214 group, the higher the fibre degradability $(37,41)$. This observation was consistent with the current study, which showed that the SCFA production (which is usually used as evaluation of fibre degradability in vitro) was also higher in ALK, CELL, PECT1, and PECT2 compared to CON. Furthermore, our study also showed that the relative abundance of Ruminococcaceae NK4A214 group, as well as Ruminococcaceae UCG-002 and Ruminococcaceae UCG-005, significantly correlated with acetic acid, propionic acid, butyric acid, and (not surprisingly) total SCFA production. Roseburia is a well-known butyrate-producing bacteria (42), and a primary degrader of $\beta$ mannans (43). The significant increase in abundance of this genus was accordance with the increased butyric acid production in the current study (Figure 6), and increased predicted abundance of beta-mannosidases (EC.3.2.1.25) in the processed RSM groups (Figure 5). Bifidobacterium is suggested to be a common cross-feeding bacteria for sugar utilization (44). Previous research has been shown that Bifidobacterium bifidum relies on the presence of a primary degrader in order to grow with either resistant starch or xylan both in vitro (45) and in vivo (46). It has also been reported that numerous Bifidobacterium species grew to higher cell densities accompanied by upregulating their respective saccharolytic pathways when grown in co-culture compared to their growth in monoculture (47). This finding was consistent with the current study, where the relative abundance of Bifidobacterium was much higher than that of other genera, especially in ALK (data not shown). Alternatively, synergy existed among genera that were targeting the same substrates, possibly by specializing in degrading different motifs within the molecule.

The relative abundance of Christensenellaceae $R$-7 group increased after enzymatic and chemical treatment, which was consistent with a previous study where rumen microbiota was fed with fibolytic enzyme-treated wheat straw (48). Christensenellaceae plays an important role in degrading fibre (49) and producing acetic and butyric acid (50). All the observations above were supported by former reports that feed processed by carbohydrase enzymes could stimulate growth of specific microbes $(8,9,51)$. It can be speculated that the pretreatment of feed stuffs with carbohydrases causes the release of hydrolysis materials (presumably oligosaccharides), which promote the chemotactic response of specific bacteria, and stimulates their attachment to feed particles, thereby leading to growth of these microbes ( 9 , 15-17). 


\section{Fibre degradation and SCFA production}

Enzymatic and chemical treatment on RSM increased the amount of acetic acid, propionic acid, butyric acid, and thereby total SCFA production (Figure 6). This observation was consistent with the predicted functional profiles related to carbohydrate metabolism, where the relative abundance of fibre breakdown and fermentation enzymes and pathways increased (Figure 5). This finding was in accordance with previous studies that addition of pectolytic enzymes improved degradability of non-starch polysaccharides (NSP) of RSM in vitro $(10)$ and in broilers $(11,12)$. Giraldo et al. also reported that supplementation of exogenous cellulase increased SCFA production (9). Previous research demonstrated that supplementation of carbohydrase on feed before feeding could increase microbial protein production, ruminal cellulolytic bacterial numbers, and ruminal fibrolytic activity (51). Thus, the findings above indicate that the enzymatic and chemical treatment on RSM could sufficiently open cell wall architecture (refer to Figure 2), to enable effective accessibility of NSP to bacterial degradation enzymes, and subsequently stimulate expression of microbial saccharolytic pathways.

No bindings of mAbs recognizing non-fucolysated XG were observed in lumen samples after the microbiota was fed with ALK or CON (Figure 7), which was unexpected as binding signals were seen in the substrates themselves prior to addition to SLIM (Figure 2). The hypothesis could be entertained that XG was immediately utilized by bacteria before our first sampling time point (after 30 minutes), or that the XG structures were unable to be recognized by the mAbs after supplementing them to lumen, due to entrapment by other molecules. Binding signals still existed at $\mathrm{t} 24$ for all mAbs that showed signal at the t0.5 time point, which indicated that these structures cannot be degraded any further or more fermentation time was needed. Glycome profiling showed that binding of mAbs recognizing each polysaccharide structure/epitope were dynamic during the $24 \mathrm{~h}$ fermentation period (Figure 7). It is not unlikely that certain polysaccharide structure, such as (hemi)cellulose and pectin, were exposed to microbes stage by stage, due to ever increasing degradation of the cell wall structures over time. For instance, bacteria should break down side-chain AG before they can utilize RG-I. However, this hypothesis should be validated in future study.

The increased in abundance of Ruminococcaceae NK4A214 group, Ruminococcaceae UCG002, and Ruminococcaceae UCG-005 in enzymatic and chemical treated RSM groups showed negative correlations with XG, RG-I, and RG-I/AG (arabinogalactan side chains of RG-I), and positively correlated with AG-1, AG-2, and pectic backbone. These observations suggested that ALK, CELL, PECT1, and PECT2 stimulated these genera to utilize XG, RGI, and arabinogalactan side chains of RG-I, and exposed AG-1, AG-2, and pectic backbone to other bacteria in the current study. The abundance of Roseburia and Succinivibrionaceae UCG-001 in enzymatic and chemical treated RSM groups had negative correlations with XG and AG-1, which suggested that the treatments on RSM stimulated these genera to degrade $\mathrm{XG}$ and AG-1. A possible model of action can be explained by the adhesion theory of Rumen Cellulolytic Bacteria (52), which supposes that the (in our case) enzymatic and chemical treatments on RSM stimulate attachments of microbes to specific polysaccharides structures (53), which lead the bacteria to degrade them. However, the mechanism of exogenous enzymes enhanced degradation of plant cell walls is complex (54), with many interrelated factors, and requires further studies. Moreover, degradation of fibres requires a plethora of 
microbial enzymes as indicated for instance by the numerous PUL-loci needed by Bacteroides thetaiotaomicron to breakdown pectin (54).

\section{Conclusion}

The present study clearly demonstrated that both enzymatic and chemical pretreatment on RSM shifted its cell wall polysaccharide structure, subsequently altering microbial community composition and functional profile compared to untreated RSM, and eventually increased fibre degradability as evaluated by SCFA production. Furthermore, glycome profiling showed that the abundance of cell wall polysaccharides were dynamically changed during fermentation, and did not continuously decrease during the fermentation period as one might expect. Our findings that ALK, CELL, PECT1 and PECT2 increased fiber degradability in RSM could help guide feed additive strategies to improve efficiency and productivity in swine industry. The current study gave insight into how feed enzyme modulate microbial status, which provides good opportunity to develop novel carbohydrase, particularly in swine feed. 


\section{References:}

1. Simbaya J, Slominski BA, Rakow G, Campbell LD, Downey RK, Bell JM. Quality characteristics of yellow-seeded Brassica seed meals: Protein, carbohydrate, and dietary fiber components. J. Agric. Food Chem. 1995;43(8):2062-6.

2. Slominski BA, Campbell LD. Non-starch polysaccharides of canola meal: Quantification, digestibility in poultry and potential benefit of dietary enzyme supplementation. J. Sci. Food Agric. 1990;53(2):175-84.

3. Carpita NC, Gibeaut DM. Structural models of primary cell walls in flowering plants: consistency of molecular structure with the physical properties of the walls during growth. PIJ. 1993;3(1):1-30.

4. Broxterman SE, Schols HA. Interactions between pectin and cellulose in primary plant cell walls. Carbohydr. Polym. 2018;192:263-72.

5. Eriksson I, Andersson R, Åman P. Extraction of pectic substances from dehulled rapeseed. Carbohydr Res. 1997;301(3-4):177-85.

6. de Vries S, Pustjens AM, Kabel MA, Salazar-Villanea S, Hendriks WH, Gerrits WJ. Processing technologies and cell wall degrading enzymes to improve nutritional value of dried distillers grain with solubles for animal feed: an in vitro digestion study. J. Agric. Food Chem. 2013;61(37):8821-8.

7. De Vries S, Pustjens A, Schols H, Hendriks W, Gerrits W. Improving digestive utilization of fiber-rich feedstuffs in pigs and poultry by processing and enzyme technologies: A review. Anim. Feed Sci. Technol. 2012;178(3-4):123-38

8. $\quad$ Giraldo L, Tejido M, Ranilla M, Ramos S, Carro M. Influence of direct-fed fibrolytic enzymes on diet digestibility and ruminal activity in sheep fed a grass hay-based diet. J. Anim. Sci. 2008;86(7):1617-23.

9. $\quad$ Giraldo L, Tejido M, Ranilla M, Carro M. Effects of exogenous cellulase supplementation on microbial growth and ruminal fermentation of a high-forage diet in Rusitec fermenters. J. Anim. Sci. 2007;85(8):1962-70.

10. Pustjens AM, de Vries S, Gerrits WJ, Kabel MA, Schols HA, Gruppen H. Residual carbohydrates from in vitro digested processed rapeseed ( Brassica napus ) meal. J Agric Food Chem. 2012;60(34):8257-63.

11. Pustjens AM, de Vries S, Schols HA, Gruppen H, Gerrits WJ, Kabel MA. Understanding carbohydrate structures fermented or resistant to fermentation in broilers fed rapeseed (Brassica napus) meal to evaluate the effect of acid treatment and enzyme addition. Poult Sci. 2014;93(4):926-34.

12. De Vries S, Pustjens A, Kabel M, Kwakkel R, Gerrits W. Effects of processing technologies and pectolytic enzymes on degradability of nonstarch polysaccharides from rapeseed meal in broilers. Poult Sci. 2014;93(3):589-98.

13. Bindelle J, Pieper R, Montoya CA, Van Kessel AG, Leterme P. Nonstarch polysaccharide-degrading enzymes alter the microbial community and the fermentation patterns of barley cultivars and wheat products in an in vitro model of the porcine gastrointestinal tract. FEMS Microbiol Ecol. 2011;76(3):553-63.

14. Torok VA, Ophel-Keller K, Loo M, Hughes RJ. Application of methods for identifying broiler chicken gut bacterial species linked with increased energy metabolism. Appl Environ Microbiol. 2008;74(3):783-91.

15. Beauchemin K, Colombatto D, Morgavi D, Yang W. Use of exogenous fibrolytic enzymes to improve feed utilization by ruminants. J. Anim. Sci. 2003;81(14_suppl_2):E37-E47.

16. Ribeiro G, Gonçalves L, Pereira L, Chaves A, Wang Y, Beauchemin K, et al. Effect of fibrolytic enzymes added to a Andropogon gayanus grass silage-concentrate diet on rumen fermentation in batch cultures and the artificial rumen (Rusitec). Animal. 2015;9(7):1153-62.

17. Ribeiro GO, Badhan A, Huang J, Beauchemin KA, Yang W, Wang Y, et al. New recombinant fibrolytic enzymes for improved in vitro ruminal fiber degradability of barley straw. J. Anim. Sci. 2018;96(9):3928-42.

18. Long C, de Vries S, Venema K. Polysaccharide source altered ecological network, functional profile, and short-chain fatty acid production in a porcine gut microbiota. Benef microbes. 2020.

19. Sáyago-Ayerdi SG, Zamora-Gasga VM, Venema K. Prebiotic effect of predigested mango peel on gut microbiota assessed in a dynamic in vitro model of the human colon (TIM-2). Food Res. Int. 2017.

20. Minekus M, Smeets-Peeters M, Bernalier A, Marol-Bonnin S, Havenaar R, Marteau P, et al. A computercontrolled system to simulate conditions of the large intestine with peristaltic mixing, water absorption and absorption of fermentation products. Appl Microbiol Biotechnol. 1999;53(1):108-14.

21. Gibson GR, Cummings JH, Macfarlane GT. Use of a three-stage continuous culture system to study the effect of mucin on dissimilatory sulfate reduction and methanogenesis by mixed populations of human gut bacteria. Appl Environ Microbiol. 1988;54(11):2750-5.

22. Klindworth A, Pruesse E, Schweer T, Peplies J, Quast C, Horn M, et al. Evaluation of general 16S ribosomal RNA gene PCR primers for classical and next-generation sequencing-based diversity studies. Nucleic Acids Res. 2013;41(1):e1-e.

23. Bolyen E, Rideout JR, Dillon MR, Bokulich NA, Abnet CC, Al-Ghalith GA, et al. Reproducible, interactive, scalable and extensible microbiome data science using QIIME 2. Nat. Biotechnol. 2019:1.

24. Callahan BJ, McMurdie PJ, Rosen MJ, Han AW, Johnson AJA, Holmes SP. DADA2: High-resolution 
sample inference from Illumina amplicon data. Nat. Methods. 2016;13:581.

25. Douglas GM, Maffei VJ, Zaneveld J, Yurgel SN, Brown JR, Taylor CM, et al. PICRUSt2: An improved and extensible approach for metagenome inference. BioRxiv. 2019:672295.

26. Pattathil S, Avci U, Baldwin D, Swennes AG, McGill JA, Popper Z, et al. A comprehensive toolkit of plant cell wall glycan-directed monoclonal antibodies. Plant Physiol. 2010;153(2):514-25.

27. Gu Z, Eils R, Schlesner M. Complex heatmaps reveal patterns and correlations in multidimensional genomic data. Bioinformatics. 2016;32(18):2847-9.

28. Pattathil S, Avci U, Miller JS, Hahn MG. Immunological approaches to plant cell wall and biomass characterization: glycome profiling. Biomass Conversion: Springer; 2012. p. 61-72.

29. Pustjens AM, Schols HA, Kabel MA, Gruppen H. Characterisation of cell wall polysaccharides from rapeseed (Brassica napus) meal. Carbohydr Polym. 2013;98(2):1650-6.

30. Siddiqui IR, Wood PJ. Carbohydrates of rapeseed: a review. J. Sci. Food Agric. 1977;28(6):530-8.

31. Pauly M, Albersheim P, Darvill A, York WS. Molecular domains of the cellulose/xyloglucan network in the cell walls of higher plants. P1J. 1999;20(6):629-39.

32. Oechslin R, Lutz MV, Amadò R. Pectic substances isolated from apple cellulosic residue: structural characterisation of a new type of rhamnogalacturonan I. Carbohydr. Polym. 2003;51(3):301-10.

33. Yatsunenko T, Rey FE, Manary MJ, Trehan I, Dominguez-Bello MG, Contreras M, et al. Human gut microbiome viewed across age and geography. nature. 2012;486(7402):222-7.

34. Cotillard A, Kennedy SP, Kong LC, Prifti E, Pons N, Le Chatelier E, et al. Dietary intervention impact on gut microbial gene richness. Nature. 2013;500(7464):585-8.

35. Flint HJ. The impact of nutrition on the human microbiome. Nutr Rev. 2012;70(suppl 1):S10-S3.

36. Nyonyo T, Shinkai T, Mitsumori M. Improved culturability of cellulolytic rumen bacteria and phylogenetic diversity of culturable cellulolytic and xylanolytic bacteria newly isolated from the bovine rumen. FEMS Microbiol Ecol. 2014;88(3):528-37.

37. Hartinger T, Edwards JE, Gómez Expósito R, Smidt H, ter Braak CJ, Gresner N, et al. Differently pretreated alfalfa silages affect the in vitro ruminal microbiota composition. Front Microbiol. 2019;10:2761.

38. Pettipher GL, Latham MJ. Production of enzymes degrading plant cell walls and fermentation of cellobiose by Ruminococcus flavefaciens in batch and continuous culture. Microbiology. 1979;110(1):29-38.

39. Flint HJ, Bayer EA, Rincon MT, Lamed R, White BA. Polysaccharide utilization by gut bacteria: potential for new insights from genomic analysis. Nat. Rev. Microbiol. 2008;6(2):121-31.

40. Shinkai T, Kobayashi Y. Localization of ruminal cellulolytic bacteria on plant fibrous materials as determined by fluorescence in situ hybridization and real-time PCR. Appl Environ Microbiol. 2007;73(5):1646-52. 41. Hartinger T, Gresner N, Südekum K-H. In vitro ruminal fermentation characteristics of alfalfa silages in response to different pre-ensiling treatments. Anim. Feed Sci. Technol. 2019;258:114306.

42. Duncan SH, Hold GL, Barcenilla A, Stewart CS, Flint HJ. Roseburia intestinalis sp. nov., a novel saccharolytic, butyrate-producing bacterium from human faeces. Int J Syst Evol Microbiol. 2002;52(5):1615-20

43. La Rosa SL, Leth ML, Michalak L, Hansen ME, Pudlo NA, Glowacki R, et al. The human gut Firmicute Roseburia intestinalis is a primary degrader of dietary $\beta$-mannans. Nat. Commun. 2019;10(1):1-14.

44. Cockburn DW, Koropatkin NM. Polysaccharide degradation by the intestinal microbiota and its influence on human health and disease. J Mol Biol. 2016;428(16):3230-52.

45. Turroni F, Özcan E, Milani C, Mancabelli L, Viappiani A, van Sinderen D, et al. Glycan cross-feeding activities between bifidobacteria under in vitro conditions. Front Microbiol. 2015;6:1030.

46. Turroni F, Milani C, Duranti S, Mancabelli L, Mangifesta M, Viappiani A, et al. Deciphering bifidobacterial-mediated metabolic interactions and their impact on gut microbiota by a multi-omics approach. The ISME journal. 2016;10(7):1656-68.

47. Milani C, Lugli GA, Duranti S, Turroni F, Mancabelli L, Ferrario C, et al. Bifidobacteria exhibit social behavior through carbohydrate resource sharing in the gut. Sci Rep. 2015;5:15782.

48. Ribeiro GO, Gruninger RJ, Jones DR, Beauchemin KA, Yang WZ, Wang Y, et al. Effect of ammonia fiber expansion-treated wheat straw and a recombinant fibrolytic enzyme on rumen microbiota and fermentation parameters, total tract digestibility, and performance of lambs. J. Anim. Sci. 2020;98(5):skaa116.

49. Mao S, Zhang M, Liu J, Zhu W. Characterising the bacterial microbiota across the gastrointestinal tracts of dairy cattle: membership and potential function. Sci Rep. 2015;5:16116.

50. Morotomi M, Nagai F, Watanabe Y. Description of Christensenella minuta gen. nov., sp. nov., isolated from human faeces, which forms a distinct branch in the order Clostridiales, and proposal of Christensenellaceae fam. nov. Int J Syst Evol Microbiol. 2012;62(1):144-9.

51. Wang Y, McAllister TA, Rode LM, Beauchemin KA, Morgavi DP, Nsereko VL, et al. Effects of an exogenous enzyme preparation on microbial protein synthesis, enzyme activity and attachment to feed in the Rumen Simulation Technique (Rusitec). Br J Nutr. 2001;85(3):325-32.

52. Miron J, Ben-Ghedalia D, Morrison M. Invited review: adhesion mechanisms of rumen cellulolytic 
bacteria. J Dairy Sci. 2001;84(6):1294-309.

53. Meale SJ, Beauchemin KA, Hristov AN, Chaves A, McAllister T. Board-invited review: opportunities and challenges in using exogenous enzymes to improve ruminant production. J. Anim. Sci. 2014;92(2):427-42.

54. Luis AS, Briggs J, Zhang X, Farnell B, Ndeh D, Labourel A, et al. Dietary pectic glycans are degraded by coordinated enzyme pathways in human colonic Bacteroides. Nat. Microbiol. 2018;3(2):210-9. 
Supplemental figures:
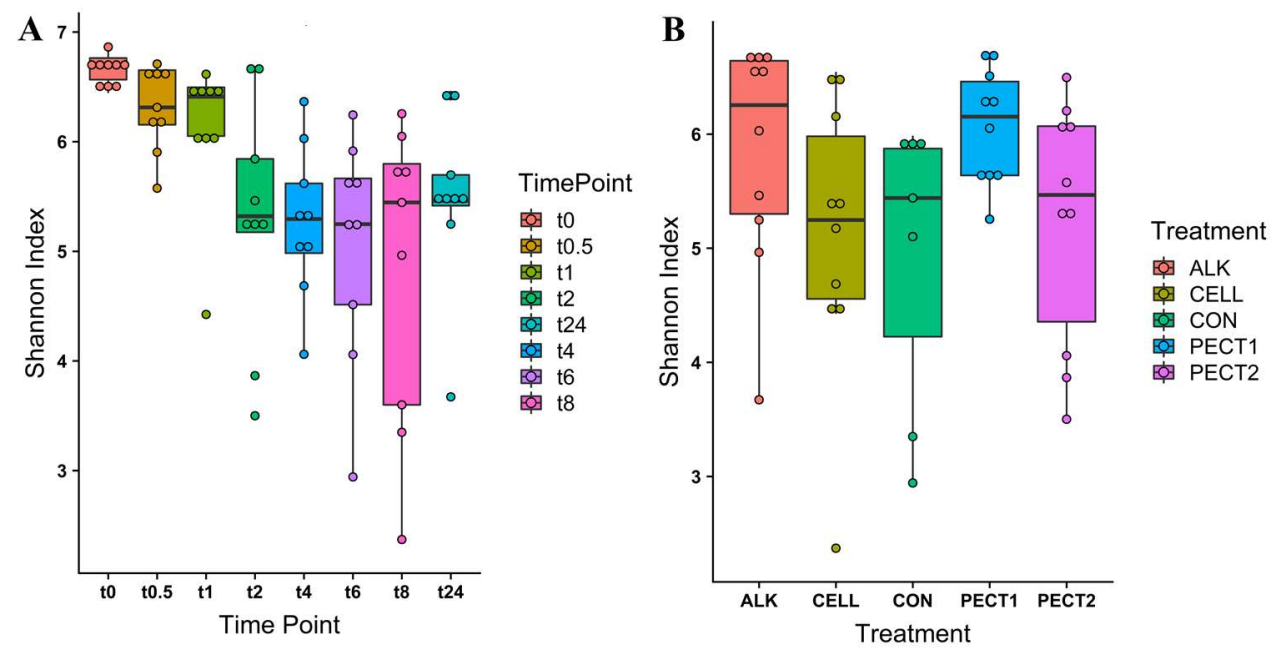

Figure S1. Community diversity represented by Shannon index at ASV level for samples from each time point (A) and treatment (B). ** $\mathrm{P}<0.01$. 


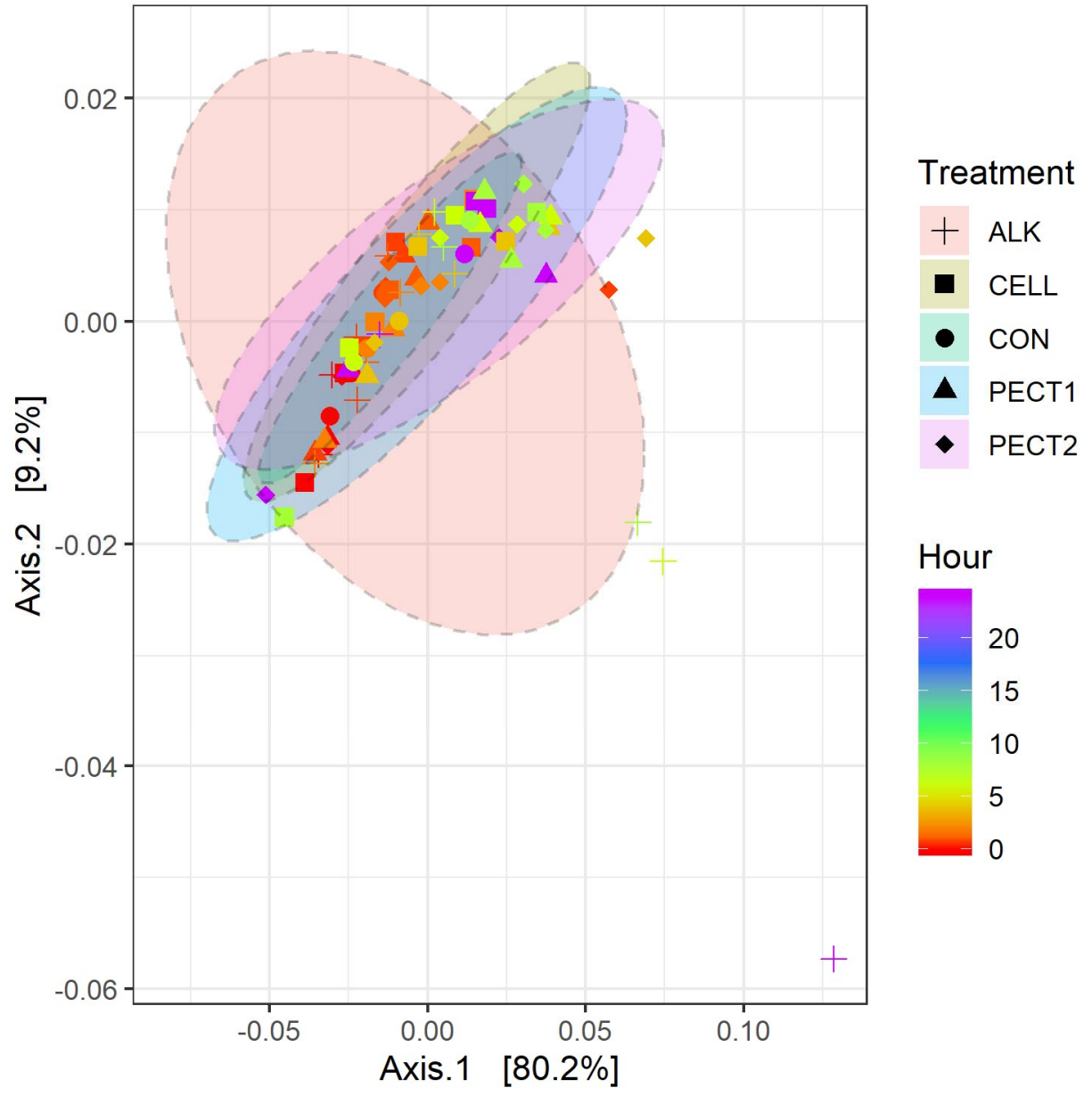

Figure S2. Principal coordinate analysis (PCoA) plot generated based on the calculated distances in weighted UniFrac matrix. Samples were grouped by shape and color in terms of treatment and time point they belonged to, respectively: CON (sphere), ALK (plus), PECT1 (triangle), PECT2 (diamond), CELL (square); a red-green-purple scale was used to indicate the fermentation time (red and purple depict start and end of the fermentation period). 


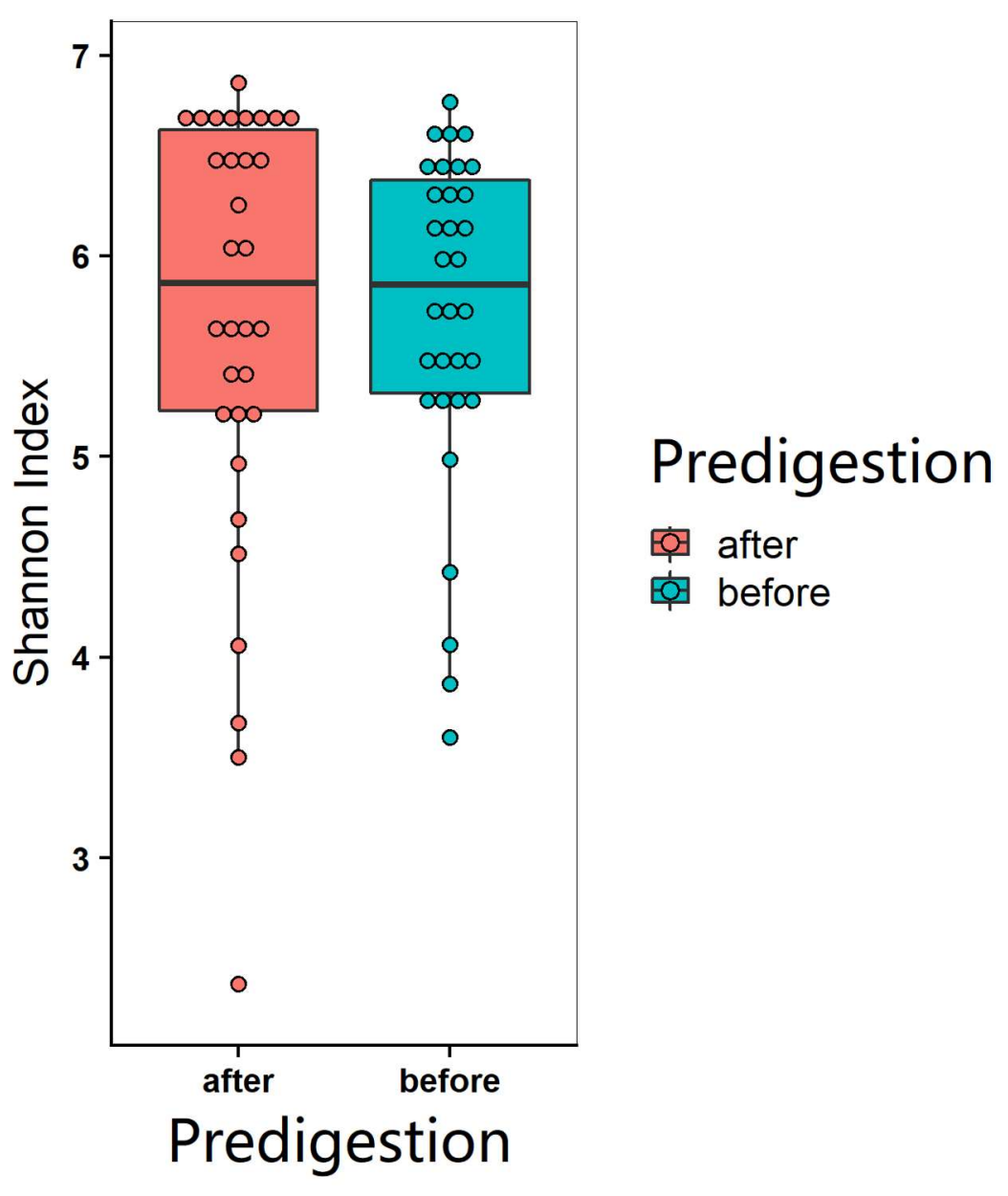

Figure S3. Community diversity represented by Shannon index at ASV level for samples from before and after predigestion. 
Chapter 3 


\title{
Chapter 4
}

\section{Differently pre-treated rapeseed meals affect in vitro swine gut microbiota composition}

\author{
Cheng Long ${ }^{1,2}$, Sonja de Vries ${ }^{3}$, Koen Venema ${ }^{1,2^{*}}$ \\ ${ }^{1}$ Faculty of Science and Engineering, Centre for Healthy Eating \& Food Innovation, \\ Maastricht University - campus Venlo, The Netherlands \\ ${ }^{2}$ School of Nutrition and Translational Research in Metabolism (NUTRIM), Maastricht \\ University, The Netherlands \\ ${ }^{3}$ Animal Nutrition Group, Wageningen University \& Research, Wageningen, The \\ Netherlands \\ "Correspondence: \\ Maastricht University - campus Venlo, Centre for Healthy Eating \& Food Innovation, St. \\ Jansweg 20, 5928 RC Venlo, the Netherlands \\ k.venema@maastrichtuniversity.nl; tel.: +31622435111
}

Published in Frontiers in Microbiology: 2020;11(2125). 


\section{Abstract}

The aim of the study was to investigate the effect of untreated and processed rapeseed meal (RSM) on fibre degradability by pig gut microbiota and the adaptation of the microbiota to the substrate, by using the Swine Large Intestine in vitro Model (SLIM). A standardized swine gut microbiota was fed for $48 \mathrm{~h}$ with pre-digested RSM which was processed enzymatically by a cellulase (CELL), two pectinases (PECT), or chemically by an alkaline (ALK) treatment. Amplicons of the V3-V4 region of the 16S rRNA gene were sequenced to evaluate the gut microbiota composition, whereas short chain fatty acids (SCFA) were measured to assess fibre degradation. Adaptive gPCA showed that CELL and ALK had larger effects on the microbiota composition than PECT1 and PECT2, and all substrates had larger effects than CON. The relative abundance of family Prevotellaceae was significantly higher in CELL treatment compared to others. Regardless of the treatments (including CON), the relative abundance of Dorea, Allisonella, and FamilyXIIIUCG_001 (in the order of Clostridiales) were significantly increased after $24 \mathrm{~h}$, and Parabacteroides, Mogibacterium, Intestinimonas, Oscillibacter, RuminococcaceaeUCG_009, Acidaminococcus, Sutterella, and Citrobacter were significantly higher in abundance at time point 48 compared to the earlier time points. Prevotella 9 had significant positive correlations with propionic and valeric acid, and Mogibacterium positively correlated with acetic and caproic acid. There was no significant difference in SCFA production between untreated and processed RSM. Overall, degradability in the processed RSM was not improved compared to CON during the RSM adaptation period. However, the significantly different microbes detected among treatments, and the bacteria considerably correlating with SCFA production might be important findings to determine strategies to shorten the fibre adaptation period of the microbiota, in order to increase feed efficiency in the animal, and particularly in pig production. 


\section{Introduction}

Rapeseed meal (RSM) is not only an important alternative feed ingredient for protein-rich feeds (where mainly soybean meal is used as protein source), but RSM is also rich in nonstarch polysaccharides (NSP) (1-3). However, a disadvantage of RSM is that a high proportion of cell wall polysaccharides cannot be utilized by endogenous enzymes from monogastric animals, and the NSP can affect the digestion of other nutrients by physical hindrance or physiological shift in the intestine (e.g. increasing digesta viscosity) (3). NSP can only be fermented in the swine large intestine by the gut microbiota, and research has shown that the pig gut microbiota needs an adaptation period to express its maximum enzymatic potential after a dietary change (4). However, most of the scientific research focused on how the fibres status affected nutrient digestibility or animal performance during the adaptation period $(5,6)$, and few monitored how the gut microbiota changed during this period (7).

Previous studies showed that a high-fibre rapeseed diet did not results in a significant increase in SCFA content in the chyme of RSM-fed pigs after a 3-week adaptation period $(7,8)$. However, SCFA-producing microbes, such as Dialister, Shuttleworthia, Bulleidia, Coprococcus, and Lachnospira, were detected more abundant in the colon of high-fibre rapeseed pigs compared to those in the control pigs (7). This might be because the 3 -week adaptation period was too short to have a considerable change in the metabolic function of SCFA-producing microbes, or more likely, the SCFA were rapidly utilized by the intestinal epithelium of the host. Adaptation duration in pigs supplemented with $19.5 \%$ palm kernel showed that 3 weeks of adaptation is needed, and a longer adaptation time is suggested as dietary palm kernel content of the diet increases (6). Huang et al. (2018) also observed that apparent total tract digestibility of acid detergent fibre significantly increased when the adaptation time increased from 7 to 28 days (6). Another report showed that microbial cellulase activity was only observed after a 6-week adaptation period when pigs were fed with different types of dietary fibre ( $8 \%$ sugar beet pulp, or $10 \%$ wheat bran) (9). Montoya et al. (2018) demonstrated that an optimum adaptation time also depends on the dietary level when growing pigs were fed with kiwi fruit (10). Thus, the optimal adaptation time should be evaluated case by case.

Treatment with carbohydrases, such as cellulase and pectinase, have been reported to improve the NSP degradation after a 4-week adaptation in both boilers and in vitro $(3,11)$. Thus, in the current study, RSM (predigested) was treated independently with two kinds of pectinases (PECT1 and PECT2), one cellulase (CELL), or alkaline (ALK), and afterwards the untreated and treated RSM preparations were fed to the recently developed Swine Large Intestine in vitro Model (SLIM) (12) for a $48 \mathrm{~h}$ period. SLIM was established on the basis of the human, computer-controlled, dynamic TNO in vitro model of the colon, TIM-2 (13), which accurately simulates the physiological conditions in the lumen of the human proximal colon, containing a gut microbiota of human origin. A unique design of the machine is that the model is equipped with a dialysate system, which prevents accumulation of microbial metabolites which would lead to the inhibition or death of microbes in the model when they would accumulate. Since all metabolites (i.e. SCFA) are collected, and basically a massbalance can be made, this allows for real determination of the production of microbial metabolites such as SCFA, which is not possible in vivo. 
In the current study, the effects of processed rapeseed meal on swine gut microbiota and SCFA production were investigated. We hypothesized that 1) the treatments on RSM will increase SCFA production (fibre degradability) during the fibre fermentation period compared to when the swine gut microbiota is fed with untreated RSM, and 2) the effects of processed RSM on the swine gut microbiota are different according to the treatments. The results of the current study give insight into how processed RSM affect pig gut microbiota during the fermentation period, which is important information to use to shorten the feed adaptation period and to improve feed efficiency. 
Materials and methods

\section{Substrate preparation}

Rapeseed meal (Brassica napus, Cargill N.V., Antwerp, Belgium; 2011) was obtained from a commercial feed mill (Agrifirm B.V., Utrecht, the Netherlands). Preparation method I (predigesting RSM after carbohydrase or alkaline treatment; Figure 1): to $200 \mathrm{~g}$ of substrate

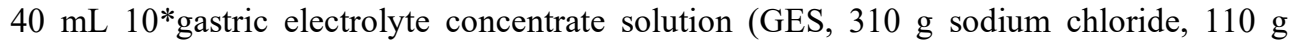
potassium chloride, $15 \mathrm{~g}$ calcium chloride di-hydrate, and $4840 \mathrm{~g}$ ultrapure water) and 360 $\mathrm{mL}$ ultrapure water were added. The $\mathrm{pH}$ was adjusted to 5.5 and then nothing (CON), ALK $(6 \mathrm{M} \mathrm{NaOH})$ or $10 \mathrm{~mL}$ of the following carbohydrases were added; CELL, Accellerase 1000 (Sigma-Aldrich, Missouri, United States); PECT1, Pectinex Ultra SP (Novozymes A/S, Bagsvaerd, Denmark); or PECT2, Multifect Pectinase (DuPont Industrial Biosciences, Genencor division, Rochester, NY). Enzyme preparations were incubated at $37{ }^{\circ} \mathrm{C}$ for $2 \mathrm{~h}$, with occasional shaking (every 30 mins), while ALK was incubated overnight at $4{ }^{\circ} \mathrm{C}$. Enzyme preparations were then heated at $100{ }^{\circ} \mathrm{C}$ for $5 \mathrm{~min}$ to inactive enzymes. Afterwards, for all five samples, $120 \mathrm{~mL}$ GES was added and the $\mathrm{pH}$ adjusted to 3 to continue with the gastric incubation according to the predigestion protocol as described elsewhere (14). After predigestion, the slurry was centrifuged $\left(8.000 \mathrm{~g}\right.$, at $4{ }^{\circ} \mathrm{C}$, for 20 minutes $)$ and dialysis was performed for the supernatants. For dialysis, a dialysis membrane (Sureflux, Nipro Europe Group Companies, Mechelen, Belgium) was used with a peristaltic pump to remove small digestion products and water. After reduction of the total volume to $\sim 450-500 \mathrm{~mL}$, supernatant was mixed with pellet, and freeze-dried. Method II (predigesting RSM before carbohydrase or alkaline treatment; Figure 1): four batches of $200 \mathrm{~g} \mathrm{RSM}$ were predigested as described before (14) and then dialyzed. Afterwards, $55 \mathrm{~mL} 10^{*} \mathrm{GES}$ was added, and $\mathrm{pH}$ adjusted to 5.5, after which $10 \mathrm{~mL}$ of CELL, PECT1, PECT2, or ALK treatment commenced, respectively. Enzyme preparations were incubated at $37{ }^{\circ} \mathrm{C}$ for 2 hours with occasional shaking (every 30 mins), and ALK was incubated overnight at $4{ }^{\circ} \mathrm{C}$. Afterwards, enzyme preparations were heated at $100{ }^{\circ} \mathrm{C}$ for $5 \mathrm{~min}$ to inactive enzymes, and $\mathrm{pH}$ was neutralized to 6.5-7 with $\mathrm{HCl}$ or $\mathrm{NaOH}$, and the samples were freeze-dried.

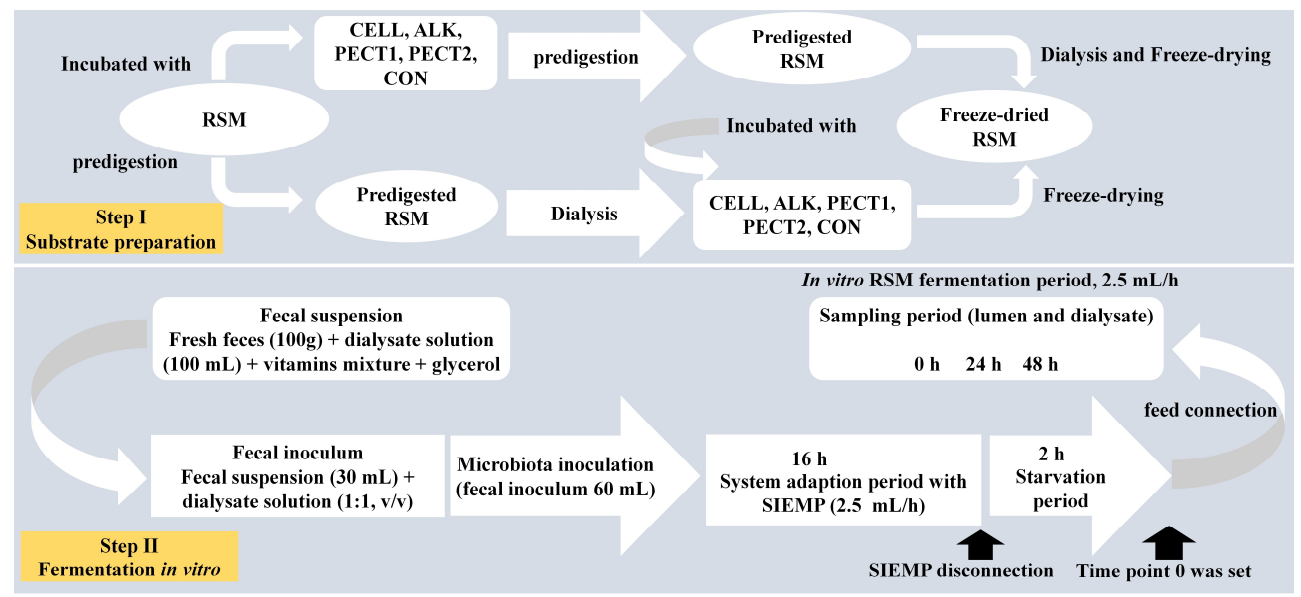

Figure 1. Schematic presentation of experimental setup for fibre fermentation in the Swine Large Intestine in vitro model (SLIM). 


\section{Fermentation in the Swine in vitro Large Intestinal Model (SLIM)}

The setup of SLIM was as follows: a fully computer-controlled in vitro model based on TIM2 (13) was used to mimic the swine large intestine (Figure S1). The pH (5.9) was controlled by continuous addition of $2 \mathrm{M}$ sodium hydroxide. Standard ileal efflux medium of pigs (SIEMP) was used to simulate the materials entering the colon. The SIEMP, adapted from (15) and described in Long et al. (12), contained the following components (g/L): 74.6 maize starch, 9.0 xylan, 19.0 pectin, 9.0 amylopectin, 9.0 arabinogalactan, 9.0 arabinoxylan, 9.0 xyloglucan, 31.5 Tween $80,43.7$ casein, 0.7 ox-bile, 43.7 bactopepton, $4.7 \mathrm{~K}_{2} \mathrm{HPO}_{4} .3 \mathrm{H}_{2} \mathrm{O}$, $0.009 \mathrm{FeSO}_{4} .7 \mathrm{H}_{2} \mathrm{O}, 8.4 \mathrm{NaCl}, 0.8 \mathrm{CaCl}_{2} .2 \mathrm{H}_{2} \mathrm{O}, 0.7 \mathrm{MgSO}_{4} .7 \mathrm{H}_{2} \mathrm{O}, 0.05$ bile, 0.02 haemin and 0.3 cysteine $\mathrm{HCl}$, plus $1.5 \mathrm{~mL}$ of a vitamin mixture containing (per litre): $1 \mathrm{mg}$ menadione, $0.5 \mathrm{mg}$ vitamin B12, $2 \mathrm{mg}$ D-biotin, $10 \mathrm{mg}$ pantothenate, $5 \mathrm{mg}$ p-aminobenzoic, $4 \mathrm{mg}$ thiamine and $5 \mathrm{mg}$ nicotinamide acid. The $\mathrm{pH}$ was adjusted to 5.9. SIEMP only contains indigestible carbohydrates and hence did not require pre-digestion. Dialysis liquid contained (per litre): $2.5 \mathrm{~g} \mathrm{~K}_{2} \mathrm{HPO}_{4} .3 \mathrm{H}_{2} \mathrm{O}, 0.005 \mathrm{~g} \mathrm{FeSO}_{4} .7 \mathrm{H}_{2} \mathrm{O}, 4.5 \mathrm{~g} \mathrm{NaCl}, 0.45 \mathrm{~g} \mathrm{CaCl}_{2} .2 \mathrm{H}_{2} \mathrm{O}, 0.05$ $\mathrm{g}$ bile, $0.5 \mathrm{~g} \mathrm{MgSO}_{4} \cdot 7 \mathrm{H}_{2} \mathrm{O}$ and $0.4 \mathrm{~g}$ cysteine $\cdot \mathrm{HCl}$, plus $1 \mathrm{~mL}$ of the vitamin mixture. All medium components were purchased at Tritium Microbiology (Eindhoven, The Netherlands). The pig fecal inoculum was a standardized microbiota from growing pigs (48 pens with 6 pigs/pen, Hypor Libra x Hypor Maxter, Hendrix Genetics, Boxmeer, The Netherlands), consisting of pooled freshly collected feces from the floor, but only material from the top (so not toughing the floor) was selected. Since this was not an intervention study, feces collection from the floor of the pins of the pigs did not require ethical approval in accordance with local/national guidelines.

In order to create a complete anaerobic environment, SLIM with $90 \mathrm{~mL}$ dialysate in each unit was flushed with gaseous nitrogen for at least 3 hours before incorporating the standardized microbiota (Figure $\mathrm{S} 1$ ). Thirty $\mathrm{mL}$ of the standardized microbiota was added to the system, making the total volume of each SLIM-unit $120 \mathrm{~mL}$. The microbiota was adapted to the model with SIEMP for 16 hours. During the adaptation phase (Figure 1) SIEMP was

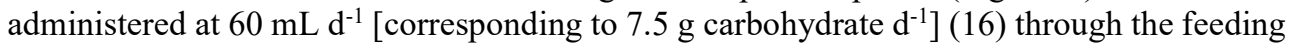
syringe (Figure S1). At the end of the adaptation period, a 2-hour starvation period was performed (Figure 1), which was used to allow all the carbohydrates within SIEMP to be fermented by the microbiota and after this time point 0 was set. Afterwards, the "fibre adaptation' period (48 hours) was performed, in which the microbiota was allowed to adapt to the test products (CON, CELL, PECT1, PECT2 and ALK). During this stage, carbohydrates in SIEMP were replaced with 7.5 grams of (treated) RSM which were added continuously in the model at a rate of $2.5 \mathrm{~mL} / \mathrm{h}$ for $48 \mathrm{~h}$ (Figure 1), corresponding to $7.5 \mathrm{~g}$ carbohydrate $\mathrm{d}^{-1}$.

\section{Sample collection}

Samples from lumen and spent dialysate were collected at time point 0,24 , and $48 \mathrm{~h}(\mathrm{t} 0$, $\mathrm{t} 24$, and 448). They were snap-frozen in liquid nitrogen and stored until analyses. Lumen samples were used to analyze microbiota composition, and both lumen and dialysis samples were analyzed for short chain fatty acid (SCFA) concentrations.

\section{Sequencing of $\mathrm{V3}-\mathrm{V} 4$ region of the $16 \mathrm{~S}$ rRNA gene}

Microbial DNA extraction and sequencing of the V3-V4 region of the 16S rRNA gene were 
performed by BaseClear B.V. (Leiden, The Netherlands). Briefly, genomic DNA extraction was performed using the Quick-DNA ${ }^{\mathrm{TM}}$ Fecal/Soil Microbe Miniprep Kit (Zymo Research, CA, USA) according to the manufacturer's instructions. Barcoded amplicons from the V3V4 region of 16S rRNA genes were generated using a 2-step PCR. 10-25 ng genomic DNA was used as template for the first PCR with a total volume of $50 \mu \mathrm{L}$ using the $341 \mathrm{~F}$ ( $5^{\prime}$ CCTACGGGNGGCWGCAG-3') and the 785R (5'-GACTACHVGGGTATCTAATCC-3') primers (17) appended with Illumina adaptor sequences. PCR products were purified (QIAquick PCR Purification Kit, Venlo, The Netherlands) and the size of the PCR products were checked on a Fragment analyzer (Advanced Analytical, Ankeny, US) and quantified by fluorometric analysis. Purified PCR products were used for the 2nd PCR in combination with sample-specific barcoded primers (Nextera XT index kit, Illumina, CA, USA). Subsequently, PCR products were purified, checked on a Fragment analyzer and quantified, followed by multiplexing, clustering, and sequencing on an Illumina MiSeq with the paired-end (2x) 300 bp protocol and indexing. The sequencing run was analyzed with the Illumina CASAVA pipeline (v1.8.3) and demultiplexed based on sample-specific barcodes. Raw sequencing data was submitted to the European Nucleotide Archive under the accession number: PRJEB38485.

\section{Bioinformatics analysis}

The demultiplexed raw sequences obtained from BaseClear were processed using QIIME2 pipeline (18). In short, reads were imported, quality filtered and dereplicated with q2-dada2 (19), after which dada2 was performed with paired-end reads and truncations parameters as follows: the first 10 base pairs were trimmed off and at position 280 base pairs the fragment was truncated in forward reads, and at position 240 base pairs for the reverse reads. The processed sequences were used for all the downstream analyses. Alpha-diversity (Shannon index) and beta diversity (weighted and unweighted UniFrac; $(20,21)$ ) were analyzed by the q2-phylogeny plugin (https://github.com/qiime2/q2-diversity).

Adaptive generalized PCA, gPCA. Adaptive gPCA $(22,23)$ was performed on the ASVtables generated in QIIME. This is a relatively new method which can obtain a lowdimensional representation of the samples in which the axes are interpretable at a fine phylogenetic scale, which can be used to compare the dissimilarities between samples more accurately than that of UniFrac. The mathematical algorithm for gPCA can be found in the literature (22). R packages biomformat, yaml, Biostrings, phyloseq, Hmisc, qiiime2R, vegan, ggplot2, and adaptiveGPCA were used in this analysis.

Phylogenetic Investigation of Communities by Reconstruction of Unobserved States, PICRUSt2. PICRUSt2 software (24) was used to predict microbial functional abundances based on marker gene sequences. KEGG database was used to predict the results.

\section{Chemical analyses}

Short-chain fatty acids analyses. Samples from lumen and dialysate were analyzed by Brightlabs (Venlo, The Netherlands) for determination of concentrations of SCFA (including the middle chain fatty acids valeric and caproic acid). Ion exclusion chromatography (IEC) was applied on an 883 Ion Chromatograph (IC; Metrohm, Herisau, Switzerland), using a Transgenomic IC Sep ICE-ION-300 column $(30 \mathrm{~cm}$ length, $7.8 \mathrm{~mm}$ diameter and $7 \mu \mathrm{m}$ particles) and a MetroSep RP2 Guard. The mobile phase consists of $1.5 \mathrm{mM}$ aqueous 
sulphuric acid. A column flow rate of $0.4 \mathrm{ml} \mathrm{min}^{-1}$ was used. The temperature of the column was $65{ }^{\circ} \mathrm{C}$. The organic acids were detected using suppressed conductivity detection. Samples were centrifuged $(14,000 \mathrm{rpm}, 10 \mathrm{~min})$, and the clear supernatant was filtered through a $0.45 \mu \mathrm{m}$ PFTE filter and diluted with mobile phase (for lumen 1:5, for dialysate 1:2). Ten microliters were loaded on the column by an autosampler 730 (Metrohm). Molecules were eluted according to their pKa.

\section{Statistical methods}

Kruskal-Wallis Rank Sum Test (one-way ANOVA on ranks) was applied to compare alpha diversities (Shannon index) among different RSM treatments and time points, and Wilcoxon Rank Sum Test was used for pairwise comparison in $\mathrm{R}$ version 3.5.3 (https://www.rproject.org/). Bonferroni was used to correct $\mathrm{P}$-values. Permutational multivariate analysis of variance (PERMANOVA; (25)) was performed to test the significance of beta diversity (weighted and unweighted UniFrac) between non-processed and processed RSM in QIIME2. The results were visualized in R. Linear discriminant analysis effect size [LEfSe] (26) was used to find biomarkers between groups using relative abundances from the feature tables generated in QIIME at genus level.

Pearson correlations between continuous meta-variables (SCFA production) and taxonomic variables were calculated and visualized in R. Parameters were set as follows: Missing values for meta-variables were handled as NO imputation (replacing missing data with substituted); zeros were kept for the calculation of correlation; a minimum relative abundance of $0.1 \%$ was considered for calculation; a minimum of 4 pairs of observations were required for calculation of correlations.

T-tests were conducted to compare SCFA production among CON, ALK, PECT1, PECT2, and CELL in the built-in R package. 


\section{Results}

RSM with different treatment methods differentially changed the microbial phylogenetical relationship during the fibre fermentation period

There were no significant difference in $\alpha$-diversity (Shannon Index) between non-processed RSM (CON) and processed RSM (ALK, PECT1, PECT2, and CELL) (Figure 2A). Alphadiversity was significantly decreased during the fibre fermentation period (Figure 2B), significant at $72 \mathrm{~h}$. Unweighted UniFrac shows that $\mathrm{CON}$ had a significant different microbial community compared to ALK, PECT1, PECT2, and CELL while there were no significant difference between non-processed and processed RSM in terms of weighted UniFrac (Figure S2). The biplot of unweighted UniFrac (Figure S2) showed that genus Prevotella 9 was mainly associated with t0, while Ruminococcaceae gauvreauii group, Eubacterium nodatum group, Succiniclasticum, Mogibacterium, Olsenella, Prevotellaceae NK3B31 group Bacteroidles S24-7 group Rikenellaceae RC9 gut group, and Ruminococcaceae UCG-002 were associated with t24 and t48. Afterwards, adaptive gPCA (23) was used to identify the dissimilarities among samples. The results show that the starting points ( $\mathrm{t} 0$ ) of all the treatments were approximately located in the same spot of the principle plane, and the later time points lay along the first adaptive gPCA axis (Figure 3A). After 48 hours adaptation, the microbiota composition changed considerably in the processed RSM groups compared to the start time points, whereas $\mathrm{CON}$ only showed a slight difference between $\mathrm{t} 48$ and t0 (Figure 3B).
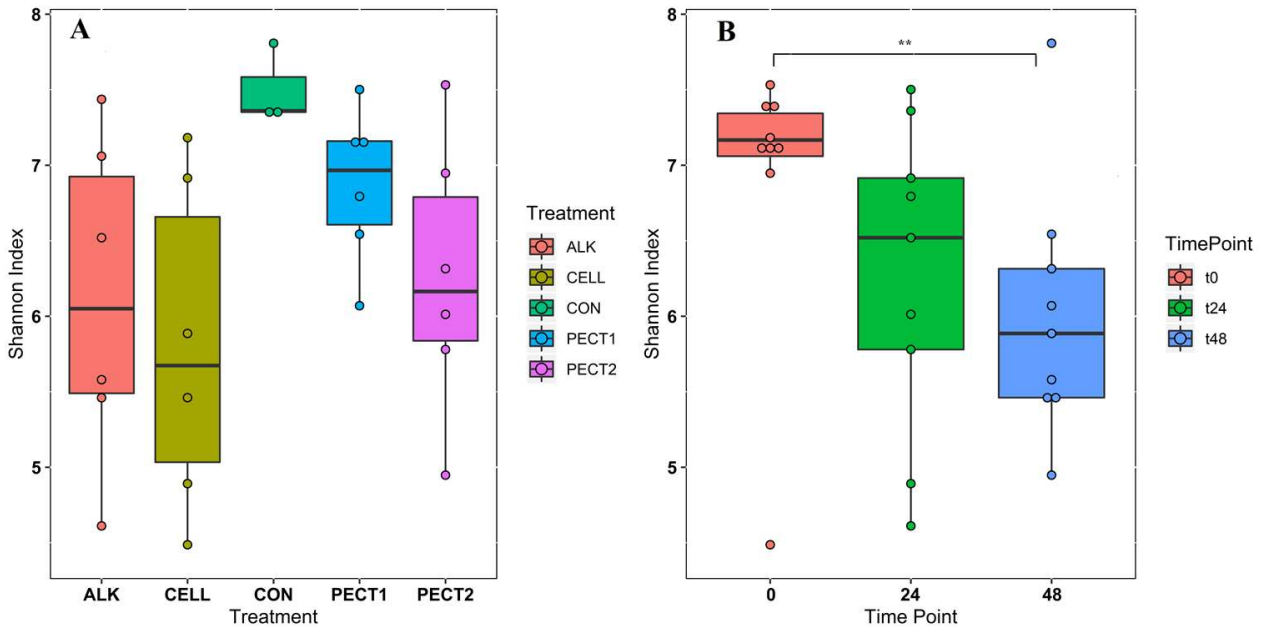

Figure 2. Community $\alpha$-diversity represented by Shannon index from each treatment (A) and time point (B). The treatments are non-processed RSM (CON) and RSM processed by Accellerase 1000 (CELL), Pectinex Ultra SP (PECT1), Multifect Pectinase (PECT2), or 6M $\mathrm{NaOH}(\mathrm{ALK})$. 

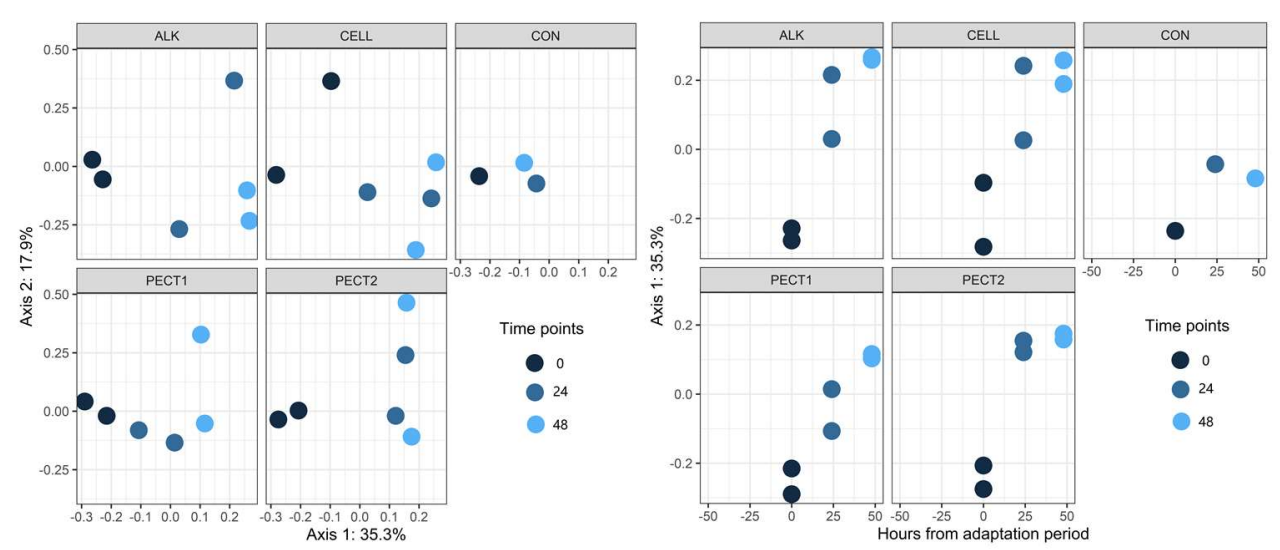

Figure 3. Two views of results from adaptive gPCA. (A) shows the sample scores from gPCA plotted on the first two axes. (B) sample scores have been centered by treatments so as to better show the within-treatment variation, and the centered scores along the first axis are displayed over time.

Alpha-diversity (Shannon index) and $\beta$-diversity (weighted and unweighted UniFrac) showed that there were no significant differences between microbiota fed with modified RSM before or after dialysis (data not shown). These indicated that predigesting before or after modifying RSM by ALK or enzymes did not differentially affect microbiome composition. Our previous study showed that predigesting before or after processing RSM had little effect on their monosaccharide constitution (Long et al. manuscript2 submitted). Therefore, they were treated as duplicates in the following microbial relative abundance analysis.

Fermentation time had larger effect on the microbiota composition during the fibre fermentation period than the treatments on RSM did

Figure 4A shows at the phylum-level that the relative abundances of Bacteroidetes in response to ALK, CON, PECT1, PECT2, and CELL were quite similar to each other at t0, whereas they continuously deceased with ALK, PECT1, and CELL feeding at both 24 and t 48 compared to $\mathrm{t} 0$, while feeding CON and PECT2 decreased the relative abundances of Bacteroidetes at $\mathrm{t} 24$, which slightly increased again at $\mathrm{t} 48$. The relative abundance of Firmicutes in ALK, CON, PECT1, and PECT2 was quite stable during the fibre fermentation period, while it continuously increased in CELL. The relative abundances of Actinobacteria in ALK, PECT1, and PECT2 increased, whereas those of CON and CELL were stable during the fibre fermentation period. ALK decreased the relative abundance of Proteobacteria, while the others treatments increased this phylum during the fibre fermentation period. 


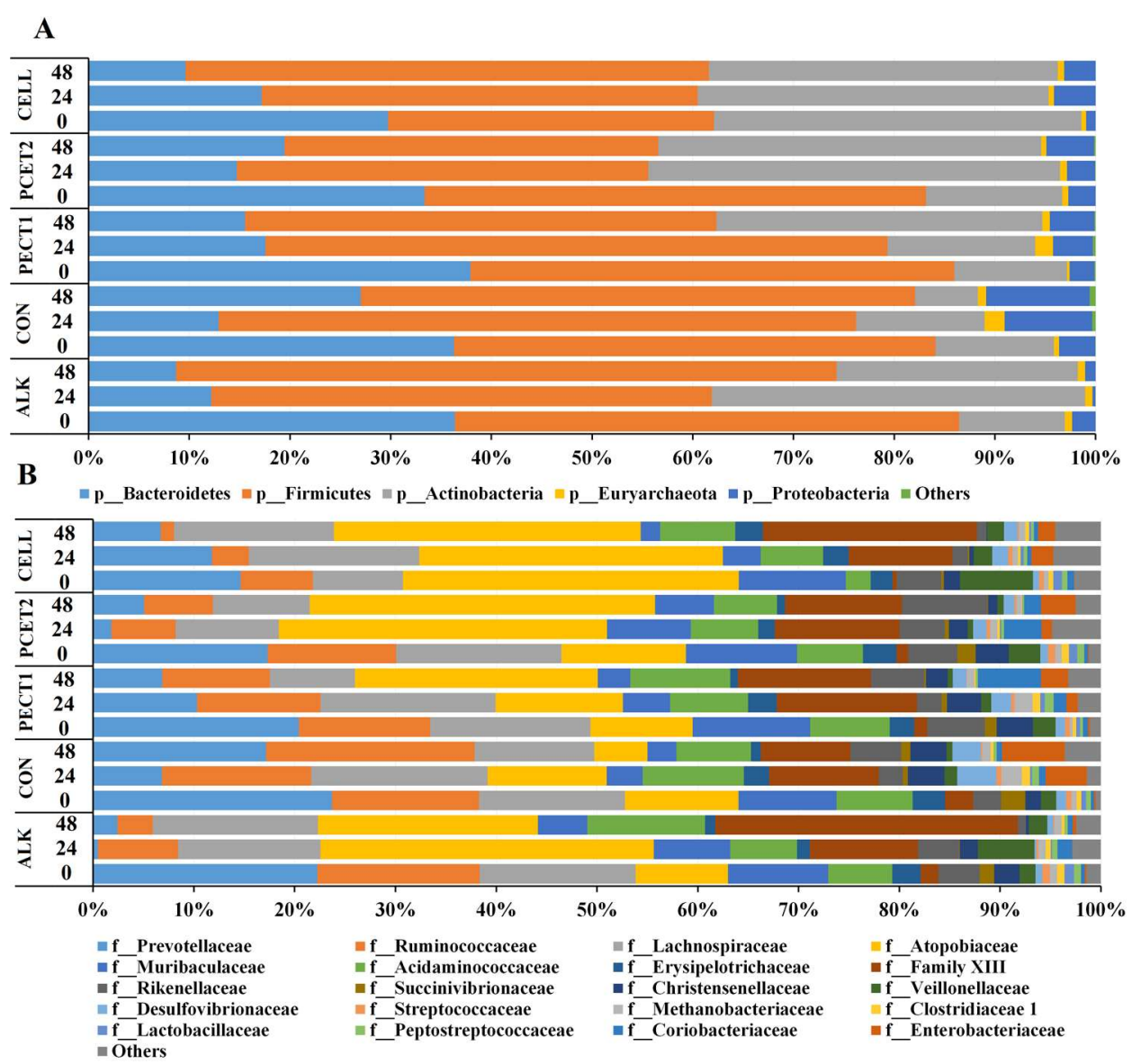

Figure 4. Relative abundances of microbial phyla (A) and families (B) in pig microbiomes fed with non-processed RSM (CON) or RSM processed by Accellerase 1000 (CELL), Pectinex Ultra SP (PECT1), Multifect Pectinase (PECT2), or 6M NaOH (ALK) at different time points $(0 \mathrm{~h}, 24 \mathrm{~h}$, and $48 \mathrm{~h})$.

Figure 4B shows at the family level that the different substrates had an effect on relative abundance at the family level, and the top six most abundant families are summarized below. ALK, PECT2, and CELL feeding decreased the relative abundance of Prevotellaceae, Ruminococcaceae, and Muribaculacceae. Lachnospiraceae was stable, while Atopobiaceae and Acidaminococaceae increased upon feeding ALK. Lachnospiraceae decreased, Atopobiaceae increased, and Acidaminococaceae was stable upon feeding PECT2. Lachnospiraceae increased, Atopobiaceae was stable, and Acidaminococaceae decreased with CELL treatment. CON and PECT1 feeding decreased the relative abundance of Prevotellaceae, Lachnospiraceae, and Muribaculacceae, and stabilized Ruminococcaceae and Acidaminococaceae. The numbers of Atopobiaceae decreased with CON, while it increased with PECT1. 
Linear discriminant analysis effect size [LEfSe] (26) was used to find the significantly different taxonomic ASVs between treatments (Figure 5A, Figure S3A) and during the fibre fermentation period (Figure 5B and Figure S3B). The relative abundance of Family Prevotellaceae was significant higher in CELL treatment compared to CON. The relative abundances of ten genera were significant higher in CON compared to CELL: Prevotella 9, Kurthia, Christensenellaceae R_7 group, Lachnoclostridium, Anaerotruncus, Ruminococcaceae NK4A214 group, Ruminococcaceae UCG_002, and Ruminococcaceae UCG_003, Ruminococcaceae UCG_005, and Ruminococcaceae UCG_010. There were no significantly different microbes in PECT1 and PECT2 treatments.
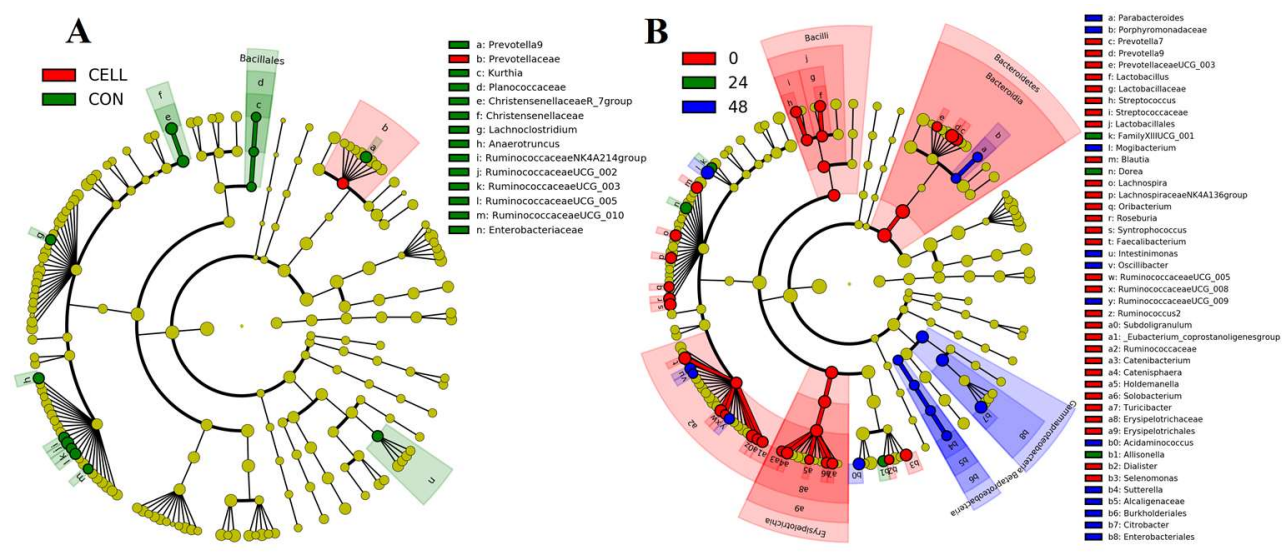

Figure 5. LEfSe results of pig microbiota fed with A) CELL and CON, and B) at time point 0,24 , and $48 \mathrm{~h}$. Differences are represented by the color of the substrates (red, green, and blue indicating CELL and time point $0 \mathrm{~h}$, time point $24 \mathrm{~h}$, and CON and time point $48 \mathrm{~h}$, for panels A and B respectively).

At genus level, more significantly different microbes were detected among the different time points (Figure 5B and Figure S3B). the relative abundance of three microbes, Dorea, Allisonella, and FamilyXIIIUCG_001 (in the order of Clostridiales), were significantly increased after $24 \mathrm{~h}$ adaptation. The relative abundance of eight microbes were significantly higher in 448 compared to the earlier time points. These were Parabacteroides, Mogibacterium, Intestinimonas, Oscillibacter, RuminococcaceaeUCG_009, Acidaminococcus, Sutterella, and Citrobacter. Figure 5B shows that the relative abundance of 24 genera (linked to t0) significantly decreased after the fibre adaptation period ( 24 and t48).

\section{Functional metagenomic profiles changed after the fibre fermentation period}

The relative abundances of PICRUSt2-predicted Enzyme Classification numbers and pathways were used to create PCA plots (Figure 6). Figure 6 shows that samples from t0 clustered together and clearly separated with samples from 124 and t48. However, there was no clearly separation among different treatments (Figure 6). 

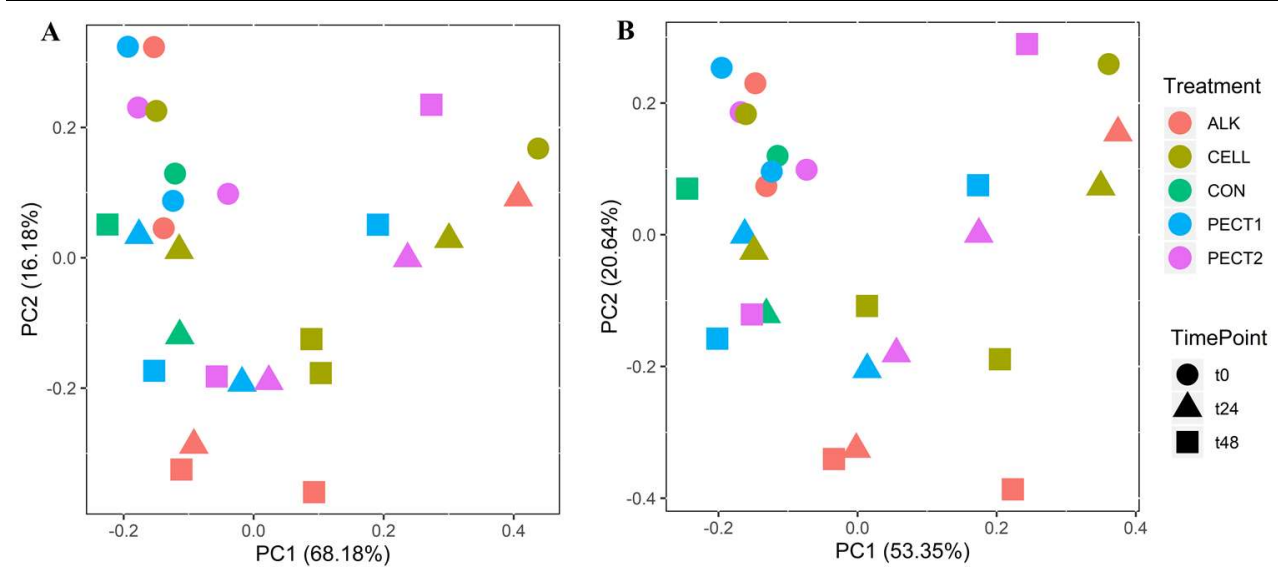

Figure 6. PCA plots of the relative abundances of Enzyme Classification numbers (A) and pathways (B) from microbiotas fed with CELL, CON, PECT1, PECT2, and ALK at different time points $(0,24,48 \mathrm{~h})$.

In this study we focused on carbohydrate metabolism related microbial functions. Figure 7 shows that 8 pathways were significantly increased after the fibre adaptation period, and they were D-galactarate degradation I, D-glucarate degradation I, glycoxylate cycle, pyruvate fermentation to acetone, TCA cycle IV (2-oxoglutarate decarboxylase), superpathway of "Dglucarate and D-galactarate degradation", superpathway of "glycolysis, pyruvate dehydrogenase, TCA, and glyoxylate bypass", and superpathway of "glycoxylate bypass and TCA". On the other hand, 4 pathways were significantly decreased after the fibre adaptation period, which were D-galacturonate degradation I, lactose and galactose degradation I, mannan degradation, and pyruvate fermentation to propanoate I. Additional significant increased other pathways and all enzymes are displayed in Table S1 and S2, respectively.
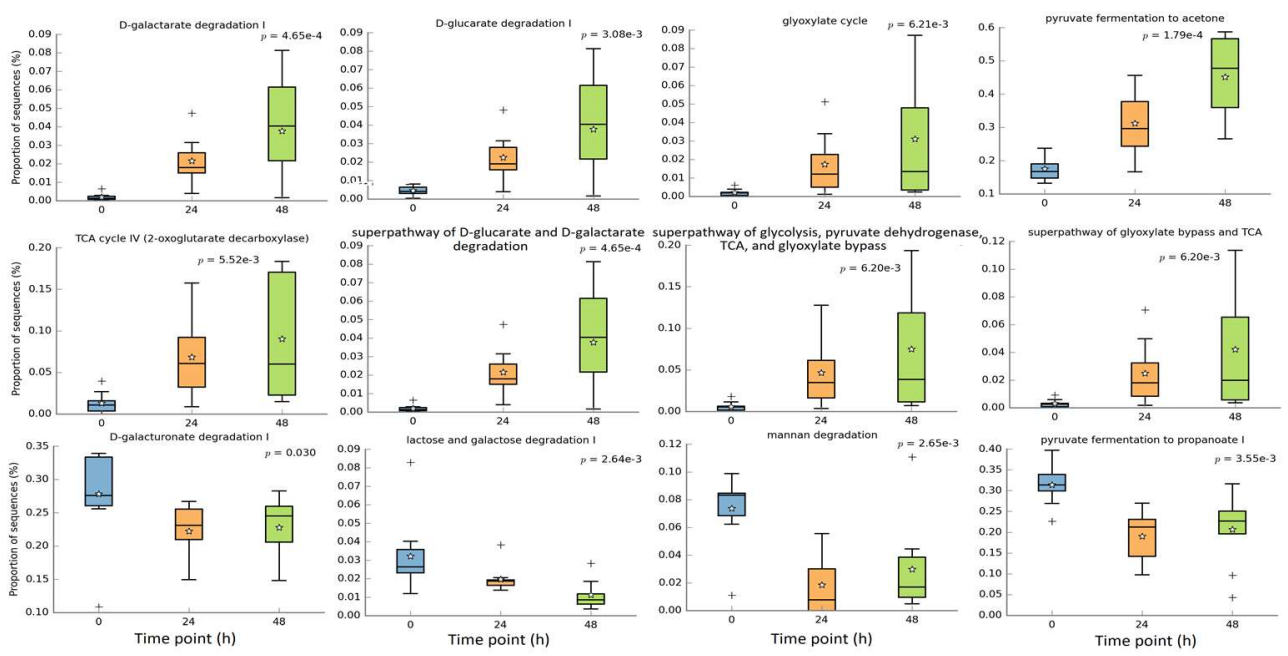
Figure 7 Significantly different metagenomic functions in relative abundance among different time points.

There were no significantly different pathways between treatments and CON.

The cumulative amount of SCFA continuously increased during the fibre fermentation period

Figure 8A shows the average cumulative amount of total, individual SCFA (acetic, propionic, butyric, and including the middle chain fatty acids valeric and caproic acid) at $\mathrm{t} 24$ and $\mathrm{t} 48$. When mentioning total SCFA or SCFA production, the middle chain fatty acids valeric and caproic acid, were included in the current context. Production of total and individual SCFA constantly increased after 24 and $48 \mathrm{~h}$ of fermentation. As generally observed, more acetic acid was produced compared to the amount of propionic and butyric acid, which were similar to each other. There were no significant difference between the first $24 \mathrm{~h}(\mathrm{t} 24)$ and second $24 \mathrm{~h}(\mathrm{t} 48)$ in SCFA production, except for propionic acid production, where the amount of propionic acid production at $\mathrm{t} 24$ was significant higher than that at $\mathrm{t} 48(\mathrm{P}<0.05)$, independent of substrate (Figure 8B). The first $24 \mathrm{~h}$ of fibre adaptation resulted in an increase of 7.0, 7.4, 7.5, 6.3 and $6.6 \mathrm{mmol}$ total SCFA/g substrate in response to ALK, CELL, CON, PECT1, and PECT2, respectively. This slightly increased during the second $24 \mathrm{~h}$ of fermentation, to 7.1, 8.2, 7.9, and $7.0 \mathrm{mmol}$ total $\mathrm{SCFA} / \mathrm{g}$ substrate in response to ALK, CELL, CON, and PECT1 feeding, respectively. Only for PECT2 the SCFA production slightly decreased during the second $24 \mathrm{~h}$ of fermentation compared to the first $24 \mathrm{~h}(5.7$ mmol SCFA/g substrate). 

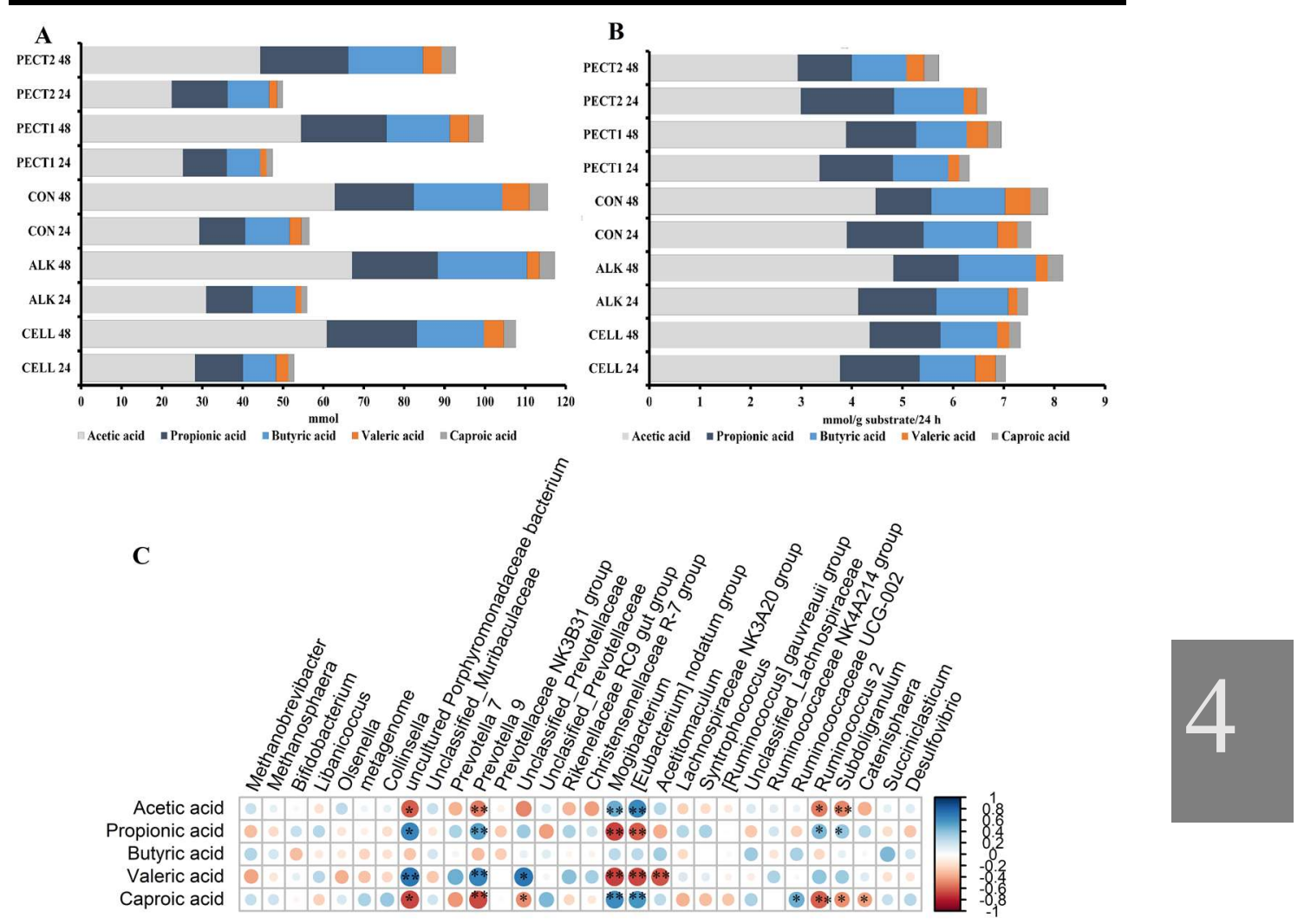

Figure 8. Average cumulative short chain fatty acid (SCFA) production in mmol (A) and SCFA increase in mmol/g substrate/24h (B) for non-processed RSM (CON) and RSM processed by Accellerase 1000 (CELL), Pectinex Ultra SP (PECT1), Multifect Pectinase (PECT2), or $6 \mathrm{M} \mathrm{NaOH}$ (ALK). The values at time point 0 were artificially set to 0 to allow determination of cumulative production. (C) correlation between SCFA production and core genera, ${ }^{*} \mathrm{p}<0.05$; blue: positive correlation; red: negative correlation. Core genera: the table was normalized via division by the sum of sequences in a given sample and multiplied by the minimum sum across all samples. Relative abundances were filtered as follows: values below a relative abundance threshold of $0.01 \%$ were not taken into account; taxa with a median relative abundance $<1 \%$ in all groups were not considered for statistical analysis.

There were no significant differences in SCFA production between unprocessed and processed RSM during the fibre fermentation period.

Correlations between the relative abundance at genus level and SCFA production were evaluated (Figure 8C). Prevotella $9\left(P_{\text {propionic acid }}<0.01, P_{\text {valeric acid }}<0.01\right)$ and uncultured Porphyromonadaceae bacterium $\left(P_{\text {propionic acid }}=0.03, P_{\text {valeric acid }}<0.01\right)$ had significant positive correlations with both propionic and valeric acid, while Mogibacterium $\left(P_{\text {propionic acid }}\right.$ $\left.<0.01, P_{\text {valeric acid }}<0.01\right)$ and $[$ Eubacterium $]$ nodatum group $\left(P_{\text {propionic acid }}=0.02, P_{\text {valeric acid }}\right.$ $<0.01)$ had negative correlation with these acids. Caproic acid significantly negatively correlated with Prevotella $9(P<0.01)$, Ruminococcus $2(P=0.03)$ and uncultured 
Porphyromonadaceae bacterium $(P=0.01)$, whereas Mogibacterium $\left(P_{\text {acetic acid }}=0.01, P_{\text {caproic }}\right.$ acid $=0.03)$ and $[$ Eubacterium $]$ nodatum group $\left(P_{\text {acetic acid }}=0.01, P_{\text {caproic acid }}=0.03\right)$ positively correlated with acetic and caproic acid. An unclassified genus from Prevotellaceae $(P<0.01)$ had positive correlation with valeric acid, while Acetitomaculum $(P<0.01)$ negatively correlated with it.

\section{Discussion}

The pig gut microbiota needs an adaptation period to express its maximum enzymatic potential after a change of diet (4). In the current study, treatment of RSM was performed prior to ingestion to decrease this adaptation period, but no significant difference was detected in SCFA production among non-processed (CON) and processed RSM (ALK, PECT1, PECT2, and CELL) fermentation during the $48 \mathrm{~h}$ fibre fermentation period in the swine in vitro large intestine model (SLIM). However, when another $24 \mathrm{~h}$ fermentation period was performed after this $48 \mathrm{~h}$ 'fibre adaptation' period, considerable changes were found in the amount of SCFA production in response to different treatments (Long et al. manuscript submitted). Previous studies show that treatments (ALK, PECT1 and PECT2) on RSM did improve the NSP degradation after 4-week adaptation in boilers and pigs $(3,27)$. Thus, it is important to know how the microbiota changes during the fibre adaptation period. The current study showed that essentially a $48 \mathrm{~h}$ period was sufficient to increase the pathways, involved in carbohydrate fermentation.

Alpha-diversity significantly decreased after $48 \mathrm{~h} \mathrm{RSM} \mathrm{fermentation,} \mathrm{while} \mathrm{there} \mathrm{were} \mathrm{no}$ significant changes between treatments and CON during the fibre adaptation period. This may due to the selection of particular microbes to utilize RSM regardless of the treatment, since RSM was the major nutrient in all cases, compared to the microbial adaptation period ( $16 \mathrm{~h}$ SIEMP adaptation), and the different treatments applied only led to changes in cell wall structures in RSM (2). In previous studies, it has been shown that RSM increased the relative abundance of Dorea and Lactobacillus in chickens (28, 29). Lachnospira, Coprococcus, Bulleidia, and Shuttleworthia are increased by RSM after three weeks adaptation in pigs (7). In the current study, a large number of genera (24 genera) significantly decreased after the fibre fermentation period, and only three and eight genera were considerably increased after adapting to the substrate for $24 \mathrm{~h}$ and $48 \mathrm{~h}$, respectively. The decreased taxa may not utilize RSM well, while RSM may be beneficial for the increased taxa. The significantly increased genera observed in the current study are Dorea, Allisonella, FamilyXIIIUCG_001 (in the order of Clostridiales), Parabacteroides, Mogibacterium, Intestinimonas, Oscillibacter, Sutterella, Citrobacter, RuminococcaceaeUCG_009, and Acidaminococcus. No overlapping genera were observed between the current study and research of Umu et al. (7), which might be because of a different pig breed. On the other hand, Dorea was found significantly increased by RSM in both the current study and research of Long et al., where six weeks of adaptation period was performed in laying hens (28).

Disappearance of some low abundance microbes were observed in either processed or nonprocessed RSM treatment since unweighted UniFrac (only considering presence or absence) was found significantly different $(P=0.02)$, while there was no difference in terms of weighted UniFrac (accounting also for abundance of observed organisms) among the treatments. This indicated that the treatments applied on RSM indeed have an effect on the microbiota composition, but that the short fibre fermentation period used may not yet have led to significant changes. In another study, we showed however that weighted UniFrac was 
observed to be significantly different among the treatments when a single shot of the fibres was introduced after this initial $48 \mathrm{~h}$ (Long et al. manuscript submitted). Zooming in to individual taxa, at the family level, Prevotellaceae significantly increased with CELL. Prevotellaceae is an important family, which is involved in degrading hemicellulose (30). We also observed that genera (e.g. Prevotella 9) from Prevotellaceae significantly positively correlated with SCFA production (Figure 7). To demonstrate the dynamic changes in swine microbiota composition in response to differently pretreated RSM, lumen samples from each time point were analyzed. The most dominant phyla were Firmicutes, Bacteroidetes, and Actinobacteria at t0, regardless of treatment (Figure 4). This was in line with previous studies (31-34), which indicated that core microbes existed. The relative abundance of Bacteroidetes decreased after supplementing with ALK, PECT1, and PECT2, whereas that of Actinobacteria increased (Figure 4). These observations implied that supplementation of ALK, PECT1, and PECT2 stimulated the growth of microbes from phylum Actinobacteria and inhibited the growth of bacteria from phylum Bacteroidetes. Actinobacteria has been reported to efficiently utilize (hemi)cellulose (35-37). Firmicutes and Proteobacteria were stimulated after supplementing with CELL (Figure 4). Firmicutes is a phylum that consists of a group of dominant butyrate-producing bacteria $(38,39)$, which play an important role in carbohydrate degradation. Research showed that the less abundant phylum, Proteobacteria, has significant functional variability in human gut microbiota (40). Zooming in to family level, ALK, PECT1, and PECT2 simulated the growth of Atopobiaceae (phylum Actinobacteria); CELL benefited the growth of Lachnospiraceae (phylum Firmicutes). The observations above suggested these microorganisms that were stimulated by these treatments play roles in the fibre degradation. Altogether, this indicated that fibre adaptation period competitively excluded low abundant microbes and created a better ecological niche for the growth of RSM-utilization (fibre fermentation) members of the microbiota.

CELL and ALK have larger effect on pig gut microbiota compared to other treatments, since their within-treatment distances between 0 and 488 were larger than those of CON, PECT1, and PECT2 (Figure 3). Our other study, with the fibre shot after $48 \mathrm{~h}$ adaptation, showed also that more genera were observed to be significantly changed particularly upon feeding CELL and ALK than with PECT1 and PECT2, when compared to CON (Long et al. manuscript submitted). The primary cell wall of RSM contains a backbone of cellulose microfibrils, which are interlinked with xyloglucan via hydrogen bonds forming a stiff network (41). Pectins are linked to each other and cross-linked with hemicellulose and cellulose (42). CELL might have relative easy access to target cellulose in RSM, whereas PECT1 and PECT2 could have less opportunity to access pectins, supposedly hindered by cellulose microfibrils. Cell wall structures processed by CELL would lead to utilization of the fibres by microbes, and increase their abundances. Alkaline pretreatment of RSM breaks the alkali-labile bonds, which are known to hinder the complete fermentation of NSP in swine, and improved NSP utilization in feed (2). However, also PECT1 and PECT2 affected the swine gut microbiota in these experiments, since gPCA showed that their distances between time point 0 and $48 \mathrm{~h}$ were larger than that of CON. A previous report showed that a cocktail of PECT1 and PECT2 improved degradability of non-starch polysaccharides of RSM in broilers (3). Treatments on RSM, either with carbohydrases or alkaline, did not improve SCFA production during the fibre fermentation period compared to non-processed RSM. This indicated that the processing applied to RSM could not improve their degradability compared to CON, in other words, they might not increase the feed efficiency during this period in vivo. It might be the 
fibre adaptation period was not enough to express its maximum enzymatic potential (4), although as mentioned earlier within $48 \mathrm{~h}$ the pathways involved in carbohydrate fermentation as increased (Figure 7A). Moreover, our own experiments with the fibre shot after $48 \mathrm{~h}$ showed that more pathways related to fibre fermentation were predicted to be upregulated (Long et al. manuscript submitted). PCA plots of Enzyme Classification numbers and pathways also show that samples from $t 0$ clustered together, but separately from samples from $\mathrm{t} 24$ and $\mathrm{t} 48$. This also indicated that microbial function between $\mathrm{t} 0$ and later time points was modulated, and geared towards degradation of these new substrates. Previous studies showed that a high-fibre rapeseed diet did not results in a significant increase in SCFA content in the chyme of RSM-fed pigs after a 3-week adaptation period $(7,8)$, which seemed to indicate that the adaptation period was not long enough. Thus, strategies should be considered to shorten the fibre adaptation period, in order to increase feed efficiency.

In conclusion, the current study demonstrated that microbiota composition was significantly affected by both RSM (processed or not) and adaptation time. Unweighted UniFrac showed that microbial community composition was significantly separated between processed RSM and CON, and CELL and ALK in general changed the microbiota composition more than PECT1 and PECT2 did. Carbohydrate metabolism related microbial functions were significantly increased after the fibre fermentation period. However, degradability of the processed RSM was not improved compared to CON during the fibre fermentation period, as assessed by SCFA production, which indicated a relative long adaptation period is needed after a diet change to RSM for swine microbiota. Thus, the significantly different microbes detected between treatments, and the bacteria considerably correlating with SCFA production might be an important finding to design specific strategies to shorten the fibre adaptation period, in order to increase feed efficiency in animals, and particularly in pig production. 


\section{References:}

1. Pustjens AM. Fate of rapeseed meal polysaccharides during digestion in pigs and poultry: effect of processing and enzyme addition. 2013.

2. Pustjens AM, de Vries S, Bakuwel M, Gruppen H, Gerrits WJ, Kabel MA. Unfermented recalcitrant polysaccharide structures from rapeseed (Brassica napus) meal in pigs. Ind Crops Prod. 2014;58:271-9.

3. De Vries S, Pustjens A, Kabel M, Kwakkel R, Gerrits W. Effects of processing technologies and pectolytic enzymes on degradability of nonstarch polysaccharides from rapeseed meal in broilers. Poult Sci. 2014;93(3):589-98.

4. Castillo M, Martín-Orúe S, Anguita M, Pérez J, Gasa J. Adaptation of gut microbiota to corn physical structure and different types of dietary fibre. Livest. Sci. 2007;109(1-3):149-52.

5. Lyu ZQ, Huang CF, Li YK, Li PL, Liu H, Chen YF, et al. Adaptation duration for net energy determination of high fiber diets in growing pigs. Anim. Feed Sci. 2018;241:15-26.

6. Huang C, Zhang S, Stein HH, Zhao J, Li D, Lai C. Effect of inclusion level and adaptation duration on digestible energy and nutrient digestibility in palm kernel meal fed to growing-finishing pigs. Asian-Australas $\mathrm{J}$ Anim Sci. 2018;31(3):395-402.

7. Umu ÖCO, Fauske AK, Åkesson CP, de Nanclares MP, Sørby R, Press CM, et al. Gut microbiota profiling in Norwegian weaner pigs reveals potentially beneficial effects of a high-fiber rapeseed diet. PloS one. 2018;13(12).

8. Chen C, Pérez de Nanclares M, Kurtz JF, Trudeau MP, Wang L, Yao D, et al. Identification of redox imbalance as a prominent metabolic response elicited by rapeseed feeding in swine metabolome1. J. Anim. Sci. 2018;96(5):1757-68.

9. Castillo M, Martín-Orúe SM, Anguita M, Pérez JF, Gasa J. Adaptation of gut microbiota to corn physical structure and different types of dietary fibre. Livest. Sci. 2007;109(1-3):149-52.

10. Montoya CA, Henare SJ, Zhu P, Rutherfurd SM, Moughan PJ. Adaptation over time of intestinal fermentation in the growing pig is influenced by the amount of kiwifruit consumed. Br J Nutr. 2018:1-43.

11. Pustjens AM, de Vries S, Gerrits WJ, Kabel MA, Schols HA, Gruppen H. Residual carbohydrates from in vitro digested processed rapeseed ( Brassica napus ) meal. J Agric Food Chem. 2012;60(34):8257-63.

12. Long C, de Vries S, Venema K. Polysaccharide source altered ecological network, functional profile, and short-chain fatty acid production in a porcine gut microbiota. Benef Microbes. 2020 .

13. Minekus M, Smeets-Peeters M, Bernalier A, Marol-Bonnin S, Havenaar R, Marteau P, et al. A computercontrolled system to simulate conditions of the large intestine with peristaltic mixing, water absorption and absorption of fermentation products. Appl Microbiol Biotechnol. 1999;53(1):108-14.

14. Sáyago-Ayerdi SG, Zamora-Gasga VM, Venema K. Prebiotic effect of predigested mango peel on gut microbiota assessed in a dynamic in vitro model of the human colon (TIM-2). Food Res. Int. 2017.

15. Gibson GR, Cummings JH, Macfarlane GT. Use of a three-stage continuous culture system to study the effect of mucin on dissimilatory sulfate reduction and methanogenesis by mixed populations of human gut bacteria. Appl Environ Microbiol. 1988;54(11):2750-5.

16. de Souza CB, Roeselers G, Troost F, Jonkers D, Koenen M, Venema K. Prebiotic effects of cassava bagasse in TNO's in vitro model of the colon in lean versus obese microbiota. J. Funct. Foods. 2014;11:210-20.

17. Klindworth A, Pruesse E, Schweer T, Peplies J, Quast C, Horn M, et al. Evaluation of general 16S ribosomal RNA gene PCR primers for classical and next-generation sequencing-based diversity studies. Nucleic Acids Res. 2013;41(1):e1-e.

18. Bolyen E, Rideout JR, Dillon MR, Bokulich NA, Abnet CC, Al-Ghalith GA, et al. Reproducible, interactive, scalable and extensible microbiome data science using QIIME 2. Nat. Biotechnol. 2019:1.

19. Callahan BJ, McMurdie PJ, Rosen MJ, Han AW, Johnson AJA, Holmes SP. DADA2: High-resolution sample inference from Illumina amplicon data. Nat. Methods. 2016;13:581.

20. Lozupone C, Knight R. UniFrac: a new phylogenetic method for comparing microbial communities. Appl Environ Microbiol. 2005;71(12):8228-35.

21. Lozupone CA, Hamady M, Kelley ST, Knight R. Quantitative and qualitative beta diversity measures lead to different insights into factors that structure microbial communities. Appl Environ Microbiol. 2007;73(5):1576-85.

22. Fukuyama J. Adaptive gPCA: A method for structured dimensionality reduction. arXiv preprint arXiv:170200501. 2017.

23. Fukuyama J, Rumker L, Sankaran K, Jeganathan P, Dethlefsen L, Relman DA, et al. Multidomain analyses of a longitudinal human microbiome intestinal cleanout perturbation experiment. PLoS Comput Biol. 2017;13(8):e1005706

24. Douglas GM, Maffei VJ, Zaneveld J, Yurgel SN, Brown JR, Taylor CM, et al. PICRUSt2: An improved and extensible approach for metagenome inference. BioRxiv. 2019:672295 
25. Anderson MJ. Permutational multivariate analysis of variance. Department of Statistics, University of Auckland, Auckland. 2005;26:32-46.

26. Segata N, Izard J, Waldron L, Gevers D, Miropolsky L, Garrett WS, et al. Metagenomic biomarker discovery and explanation. Genome biology. 2011;12(6):R60.

27. Pustjens AM, de Vries S, Bakuwel M, Gruppen H, Gerrits WJ, Kabel MA. Degradability of rapeseed meal polysaccharides in pigs. Fiber fermentation in pigs and poultry Sense and nonsense of its manipulation.2014;64.

28. Long $\mathrm{C}$, Wang J, Zhang $\mathrm{H}$, Wu S, Qi G. Effects of dietary rapeseed meal supplementation on cecal microbiota in laying hens with different flavin-containing monooxygenase 3 genotypes. Poult Sci. 2017;96(6):174858.

29. Chiang G, Lu W, Piao X, Hu J, Gong L, Thacker P. Effects of feeding solid-state fermented rapeseed meal on performance, nutrient digestibility, intestinal ecology and intestinal morphology of broiler chickens. AsianAustralas J Anim Sci. 2009;23(2):263-71.

30. Emerson EL, Weimer PJ. Fermentation of model hemicelluloses by Prevotella strains and Butyrivibrio fibrisolvens in pure culture and in ruminal enrichment cultures. Appl. Microbiol. Biotechnol. 2017;101(10):426978.

31. Casellas NM, Berri EM, Fabrellas JE, Levenez F, Lemonnier G, Denis C, et al., editors. Establishment of the swine gut microbiome during early life and further impact on adult health and performance. 34 International Society for Animal Genetics Conference; 2014.

32. Costa MO, Chaban B, Harding JCS, Hill JE. Characterization of the fecal microbiota of pigs before and after inoculation with "Brachyspira hampsonii". PLoS One. 2014;9(8):e106399.

33. Cantu-Jungles TM, do Nascimento GE, Zhang X, Iacomini M, Cordeiro LM, Hamaker BR. Soluble xyloglucan generates bigger bacterial community shifts than pectic polymers during in vitro fecal fermentation. Carbohydr. Polym. 2019;206:389-95.

34. Ramayo-Caldas Y, Mach N, Lepage P, Levenez F, Denis C, Lemonnier G, et al. Phylogenetic network analysis applied to pig gut microbiota identifies an ecosystem structure linked with growth traits. The ISME journal. 2016;10(12):2973

35. Munk C, Lapidus A, Copeland A, Jando M, Mayilraj S, Del Rio TG, et al. Complete genome sequence of Stackebrandtia nassauensis type strain (LLR-40K-21 T). Standards in genomic sciences. 2009;1(3):292-9.

36. Rastogi G, Bhalla A, Adhikari A, Bischoff KM, Hughes SR, Christopher LP, et al. Characterization of thermostable cellulases produced by Bacillus and Geobacillus strains. Bioresour Technol. 2010;101(22):8798-806.

37. Wang C, Dong D, Wang H, Müller K, Qin Y, Wang H, et al. Metagenomic analysis of microbial consortia enriched from compost: new insights into the role of Actinobacteria in lignocellulose decomposition. Biotechnol. Biofuels. 2016;9(1):22.

38. Sheridan PO, Martin JC, Lawley TD, Browne HP, Harris HM, Bernalier-Donadille A, et al. Polysaccharide utilization loci and nutritional specialization in a dominant group of butyrate-producing human colonic Firmicutes. Microbial genomics. 2016;2(2).

39. La Rosa SL, Leth ML, Michalak L, Hansen ME, Pudlo NA, Glowacki R, et al. The human gut Firmicute Roseburia intestinalis is a primary degrader of dietary $\beta$-mannans. Nat. commun. 2019;10(1):1-14.

40. Bradley PH, Pollard KS. Proteobacteria explain significant functional variability in the human gut microbiome. Microbiome. 2017;5(1):36.

41. Carpita NC, Gibeaut DM. Structural models of primary cell walls in flowering plants: consistency of molecular structure with the physical properties of the walls during growth. PIJ. 1993;3(1):1-30.

42. Broxterman SE, Schols HA. Interactions between pectin and cellulose in primary plant cell walls. Carbohydr. Polym. 2018;192:263-72. 
Supplemental figures:
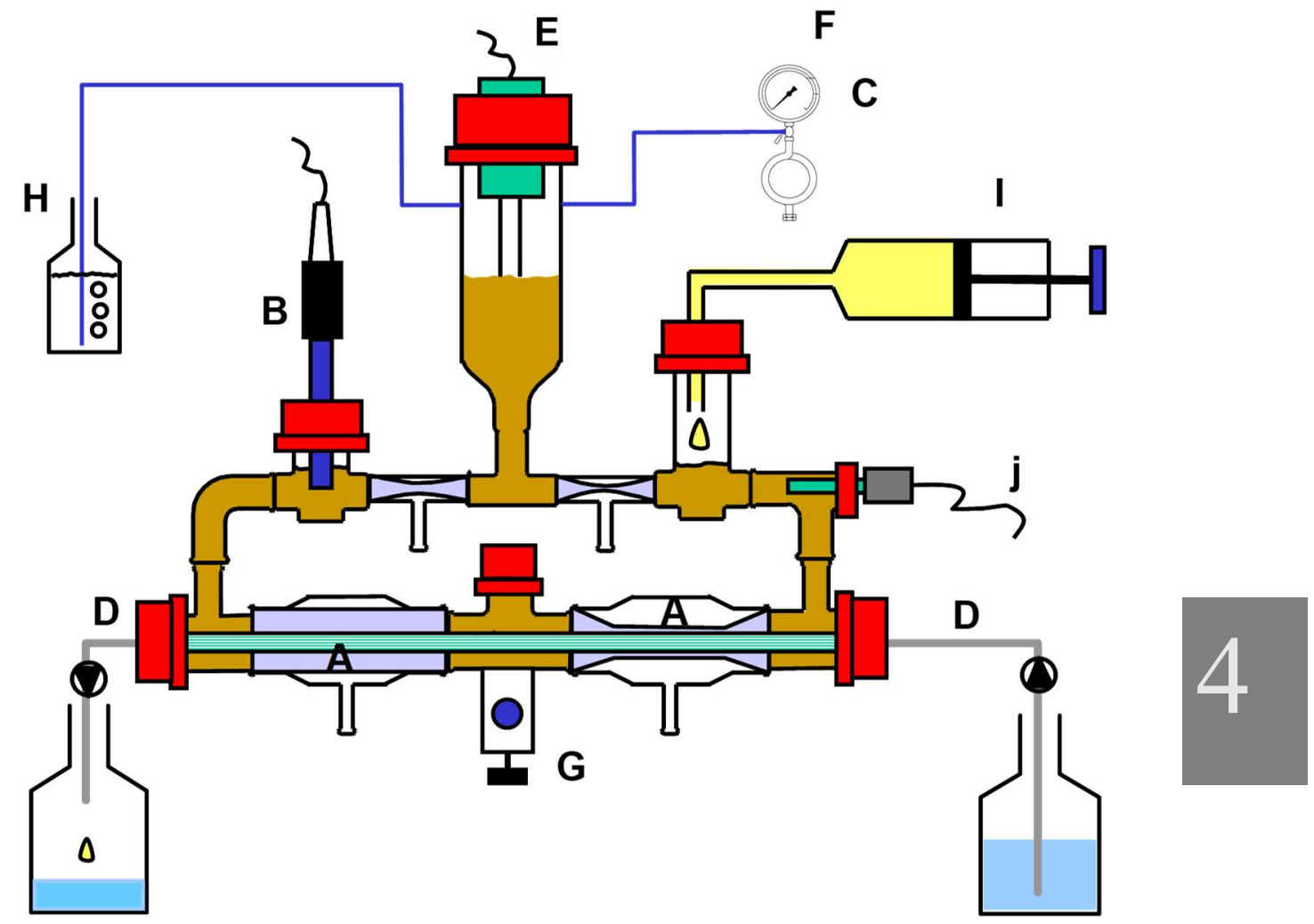

Figure S1 SLIM system. A. peristaltic compartments; B. pH-electrode; C. alkali pump; D. dialysis liquid circuit with hollow fibers; E. level-sensor; F. $\mathrm{N}_{2}$ gas inlet; G. sampling port; H. gas outlet; I. 'ileum efflux' container, or, food syringe; J. temperature sensor. 

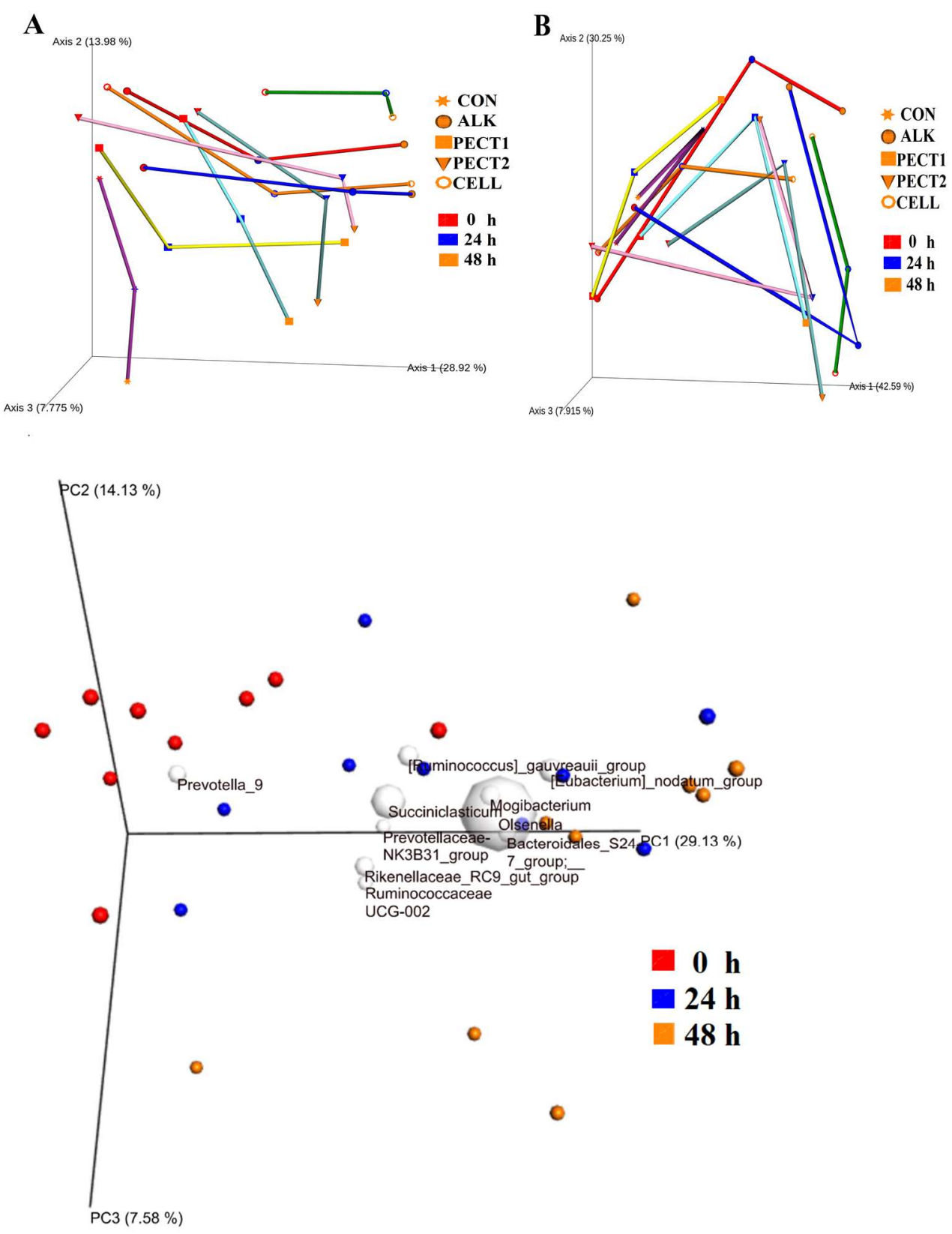

Figure S2. Principle coordinate analysis ( $\mathrm{PCoA})$ plots generated based on the calculated distances in unweighted (A) and weighted (B) UniFrac matrices. C: Biplot of unweighted UniFrac. Samples were grouped by shape and color in terms of treatment and time point they belonged to, respectively: non-modified RSM (CON) star, RSM modified by $6 \mathrm{M} \mathrm{NaOH}$ (ALK) sphere, RSM modified by Pectinex Ultra SP (PECT1) square, RSM modified by Multifect Pectinase (PECT2), triangle, and RSM modified by Accellerase 1000 (CELL), ring; time point $0 \mathrm{~h}$ (red), $24 \mathrm{~h}$ (blue), $48 \mathrm{~h}$ (orange). The trajectories started from time point $0 \mathrm{~h}$, and ended with time point $48 \mathrm{~h}$. 
Rapeseed meal in response to swine gut microbiota
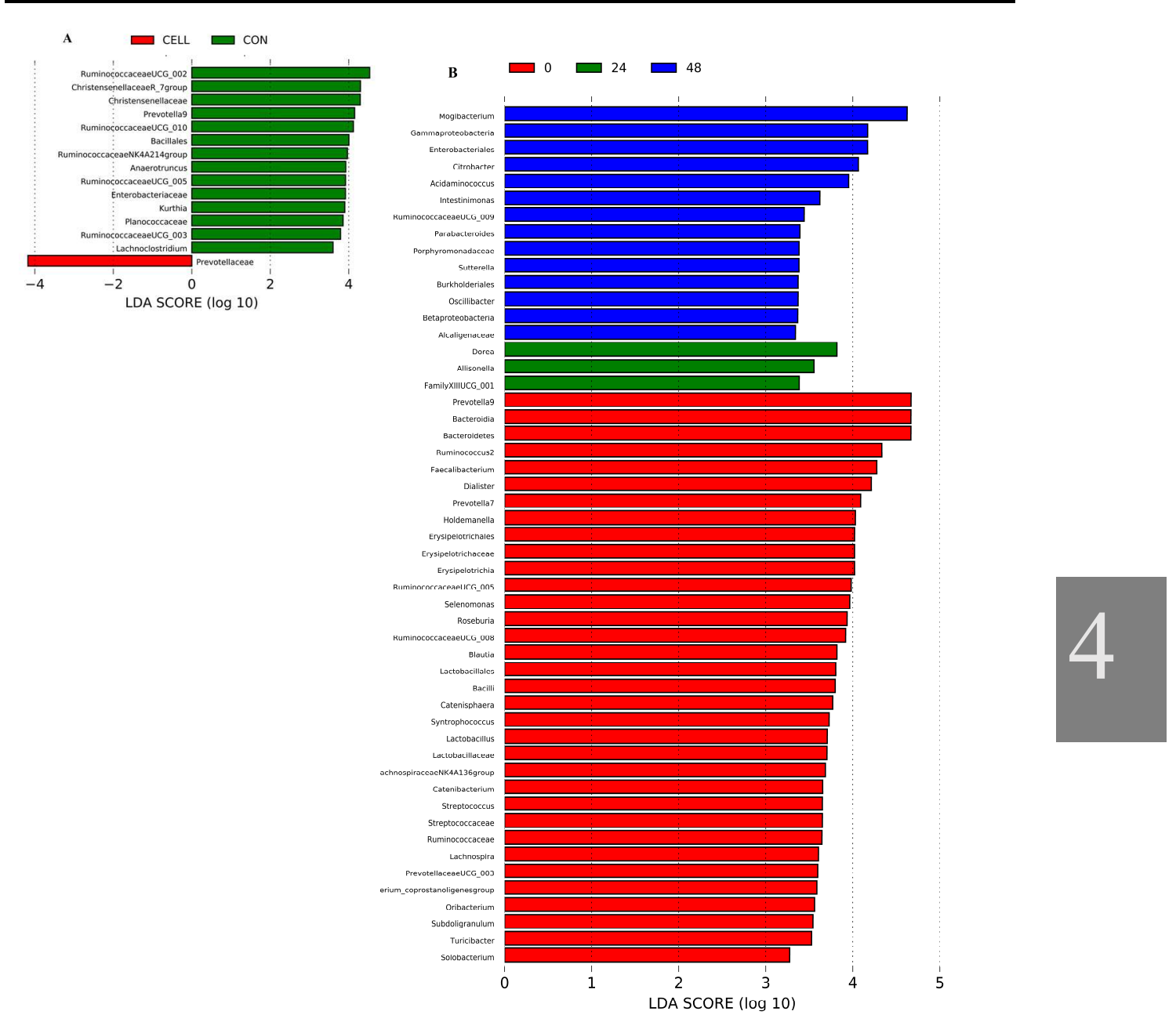

Figure S3. LEfSe results of pig microbiota fed with (A) CELL and CON, and (B) at time point 0,24 , and $48 \mathrm{~h}$. 
Chapter 4 


\title{
Chapter 5
}

\section{Cellulase and alkaline treatment improve intestinal microbial degradation of recalcitrant fibres of rapeseed meal in pigs}

\author{
Cheng Long ${ }^{1,2}$, Christiane Rösch ${ }^{3}$, Sonja de Vries ${ }^{4}$, Henk Schols ${ }^{3}$, Koen Venema ${ }^{1,2 *}$ \\ ${ }^{1}$ Faculty of Science and Engineering, Centre for Healthy Eating \& Food Innovation, \\ Maastricht University - campus Venlo, The Netherlands \\ ${ }^{2}$ School of Nutrition and Translational Research in Metabolism (NUTRIM), Maastricht \\ University, The Netherlands \\ ${ }^{3}$ Laboratory of Food Chemistry, Wageningen University \& Research, Wageningen, The \\ Netherlands \\ ${ }^{4}$ Animal Nutrition Group, Wageningen University \& Research, Wageningen, The \\ Netherlands \\ *Correspondence: \\ Maastricht University - campus Venlo, Centre for Healthy Eating \& Food Innovation, St. \\ Jansweg 20, 5928 RC Venlo, The Netherlands \\ k.venema@maastrichtuniversity.nl; tel.: +31622435111
}

Published in Journal of Agricultural and Food Chemesitry: 2020, 68(39) 11011-11025. 
ABSTRACT The aim of current study was to investigate whether degradation of rapeseed meal (RSM) by a swine gut microbiota consortium was improved by modifying RSM by treatment with cellulase (CELL), two pectinases (PECT), or alkaline (ALK) compared to untreated RSM and to assess whether microbiota composition and activity changed. The predicted relative abundances of carbohydrate digestion and absorption, glycolysis, pentose phosphate pathway, and pyruvate metabolism were significantly increased upon CELL and ALK feeding, and CELL and ALK also exhibited increased total short-chain fatty acid (SCFA) production compared to CON. Megasphaera, Prevotella, and Desulfovibrio were significantly positively correlated with SCFA production. Findings were validated in ileal cannulated pigs, which showed that CELL and ALK increased fibre degradation of RSM. In conclusion, CELL and ALK rather than PECT1 or PECT2 increased fibre degradation in RSM, and this information could guide feed additive strategies to improve efficiency and productivity in the swine industry. 


\section{Introduction}

The European Union (EU) is highly dependent on imports of protein-rich animal feed ingredients $(70 \%)$. This percentage is even higher when the focus is on soya beans alone, where the EU imports $95 \%$ of its demand or on average 36.1 million tons of soybean equivalent on a yearly basis ${ }^{1}$. Of these, 9 million tons of soybean meal are annually used in pig production. In order to have a more sustainable supply of responsible protein-rich feed ingredients, the European livestock sector needs an alternative local protein feed ingredient to fill the "protein gap".

Rapeseed meal (RSM), a byproduct from rapeseed oil production, is not only a suitable protein source for swine feed but also a potentially energy source. RSM contains a high amount of cell wall polysaccharides, even higher when compared to soybean meal commonly used in the feed industry. Non-starch polysaccharides (NSP) constitute 20 to $40 \%$ of RSM 2-3, which are represented by pectic polysaccharides (homogalacturonan, rhamnogalacturonan, arabinogalactan and arabinan), cellulose, and hemicelluloses (xyloglucan, galactomannan, and glucuronoxylan) ${ }^{4-5}$. A drawback of RSM is that the complex cell wall polysaccharides cannot be utilized by endogenous enzymes from monogastric animals, and also can only partly be fermented by the microbial community in the gastrointestinal tract (GIT). Reports show that NSP can only be degraded for 3 to $6 \%$ in chicken $^{3,6-7}$, and around 58 to $68 \%$ in pigs, which is rather low compared to other NSP-rich feed ingredients, such as sugar beet pulp (approximately $85 \%$ of NSP is degraded by pigs) ${ }^{8}$. Thus, pre-treatment of RSM is needed to improve its digestibility and fermentability. Usage of carbohydrases, e.g. $\beta$-glucanases, xylanases, cellulase and/or pectinase, is common in poultry feed, however less feed enzymes are used in pig diets with respect to increasing fibre degradation.

Meanwhile, the intestinal microbiota plays a critical role in host nutrition, health, performance and quality of meat products, since the microbiota in the GIT can degrade the undigested substrates, and create SCFA and oligosaccharides from cell wall NSP, acting as an additional energy source and having a potential prebiotic effects, respectively ${ }^{9}$. The chemical composition and structure of the substrates largely determine the (changes in) microbial composition of the bacterial community in the GIT, as microbes differ in their substrate preferences (degradation capacity) and growth requirements ${ }^{10-11}$. As a result, microbial composition and metabolic function are very much dependent on biochemical conditions of digesta. Torok and colleagues 12 investigated changes in gut microbial population in response to the supplementation of an NSP-degrading enzyme (containing $\beta$ glucanase, xylanase, and protease activities) in a barley-based diet in chickens, and the results showed that microbial composition revealed distinct clusters correlating with unsupplemented and enzyme supplemented birds. Supplementation of carbohydrases has been shown to modulate gut microbiota in a limited number of studies, both in animal and in vitro models ${ }^{13-15}$.

In the current study, RSM (predigested with digestive enzymes) was treated independently with two kinds of pectinases (PECT1 and PECT2), one cellulase (CELL), or alkaline (ALK), and afterwards the untreated and treated RSM preparations were fermented in the Swine Large Intestine in vitro Model (SLIM) ${ }^{16}$. and in vivo (in ileal cannulated pigs). We hypothesized that 1) carbohydrase increases NSP degradability of RSM, and 2) feed 
enzymes-treated RSM differentially affects the pig gut microbiota composition and through this affects the predicted microbial functional profile and potential energy yield of the substrate. The $16 \mathrm{~S}$ rRNA gene sequencing technology was used to monitor the microbial communities. The results of the current study provide insight into how carbohydrases affect swine gut microbiota, which is important information to exploit (new) feed enzymes. 
Materials and methods

\section{Substrate preparation}

Rapeseed meal (Brassica napus, Cargill N.V., Antwerp, Belgium) was obtained from a commercial feed mill (Agrifirm B.V., Utrecht, the Netherlands). Preparation method I (predigestion of RSM after carbohydrase or alkaline treatment) [Figure 1] was as follows: to

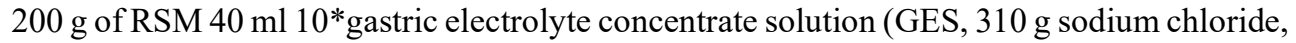
$110 \mathrm{~g}$ potassium chloride, $15 \mathrm{~g}$ calcium chloride di-hydrate, and $4840 \mathrm{~g}$ ultrapure water) and $360 \mathrm{~g} / \mathrm{mL}$ ultrapure water were added. The $\mathrm{pH}$ was adjusted to 5.5 and then nothing (CON), $10 \mathrm{~mL}$ of alkaline (ALK, $6 \mathrm{M} \mathrm{NaOH})$, or the following carbohydrases were added: CELL (Accellerase 1000, Sigma-Aldrich, Missouri, United States), PECT1 (Pectinex Ultra SP, Novozymes A/S, Bagsvaerd, Denmark), or PECT2 (Multifect Pectinase, DuPont Industrial Biosciences, Genencor division, Rochester, NY). Enzyme/substrate mixtures were incubate at $37{ }^{\circ} \mathrm{C}$ for $2 \mathrm{~h}$, with occasional shaking (every $30 \mathrm{mins}$ ), while ALK was incubated overnight at $4{ }^{\circ} \mathrm{C}$. Enzyme/substrate mixtures were then heated at $100{ }^{\circ} \mathrm{C}$ for $5 \mathrm{~min}$ to inactive enzymes. For all treatments, $\mathrm{pH}$ was neutralized to 6.5-7 with $\mathrm{HCl}$ or $\mathrm{NaOH}$. Afterwards, for all five samples, $120 \mathrm{~mL}$ GES was added and $\mathrm{pH}$ adjusted to 3 to continue with the gastric incubation according to the predigestion protocol as described elsewhere ${ }^{17}$. After predigestion, the slurry was centrifuged $\left(8,000 \mathrm{~g}, 4^{\circ} \mathrm{C}, 20 \mathrm{~min}\right)$ and dialysis was performed for the supernatants. For dialysis, a dialyzer (Sureflux, Nipro Europe Group Companies, Mechelen, Belgium) was used with a peristaltic pump to remove small digestion products and water. After reduction of the total volume to $\sim 450-500 \mathrm{~mL}$, supernatant was mixed with the pellet, and freeze-dried. For method II (digestion of RSM before carbohydrase or alkaline treatment) [Figure 1], four quantities of $200 \mathrm{~g}$ RSM were predigested as described above and then dialyzed. Afterwards, $55 \mathrm{~mL} \mathrm{10*GES} \mathrm{was} \mathrm{added,} \mathrm{and} \mathrm{pH}$ adjusted to 5.5, after which $10 \mathrm{~mL}$ of CELL, PECT1, PECT2, or ALK treatment commenced, respectively. Enzyme/substrate mixtures were incubate at $37^{\circ} \mathrm{C}$ for 2 hours with occasional shaking (every 30 mins), and ALK was incubated overnight at $4{ }^{\circ} \mathrm{C}$. Afterwards, enzyme/substrate mixtures were heated at $100{ }^{\circ} \mathrm{C}$ for $5 \mathrm{~min}$ to inactive enzymes, and $\mathrm{pH}$ was neutralized to $6.5-7$ with $\mathrm{HCl}$ or $\mathrm{NaOH}$. Samples from both method I and method II were subsequently freeze-dried. Samples are differentiated by the suffix_B (for before) or_A (for after) (e.g. PECT1_A) for carbohydrase- or ALK-treatment prior to and after digestion, respectively. 


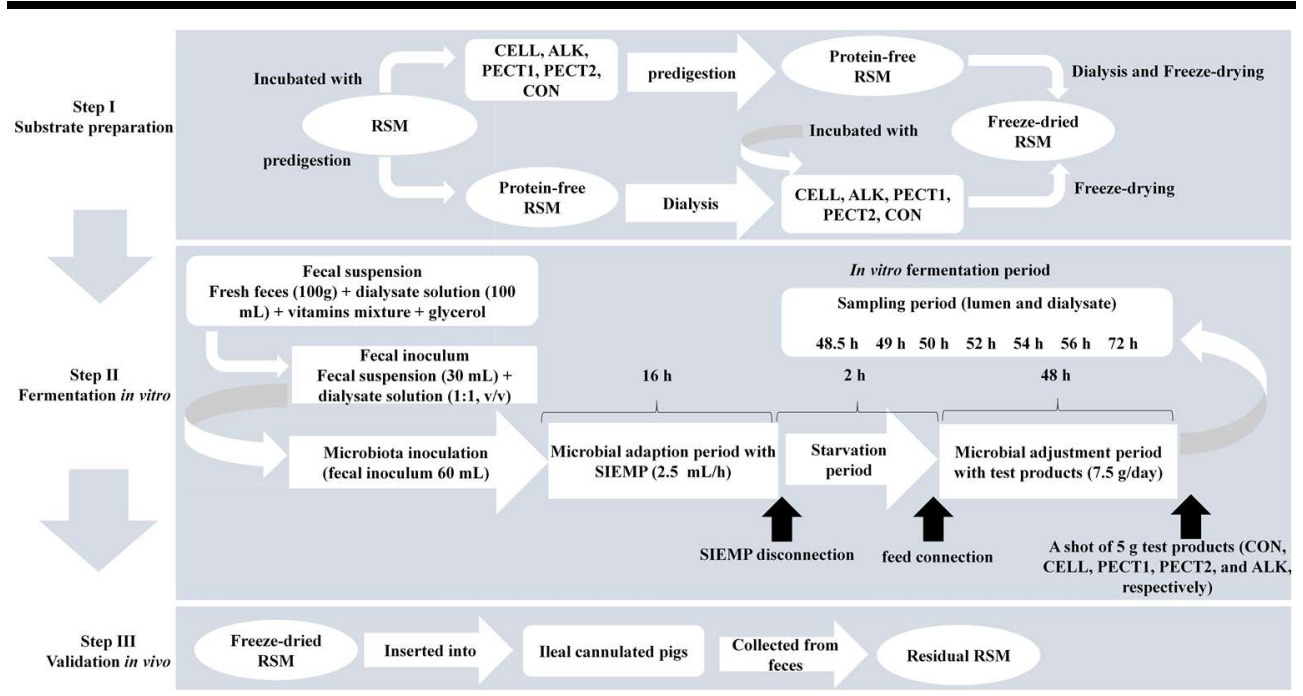

Figure 1. Schematic of experimental design.

\section{Fermentation in The Swine in vitro Large Intestinal Model (SLIM)}

The setup of SLIM has been described before (Long et al., 2020). Briefly, a fully computercontrol in vitro model was used to mimic the swine large intestine. The $\mathrm{pH}$ (5.9) was controlled by the addition of $2 \mathrm{M}$ sodium hydroxide. Standard ileal efflux medium of pigs (SIEMP) was used to simulate the materials entering the colon ${ }^{16}$. SIEMP and dialysate solution are described in detail in Long et al. ${ }^{16}$. Briefly, The SIEMP, slightly modified from ${ }^{18}$ and described in ${ }^{19}$ contained the following components $(\mathrm{g} / \mathrm{L})$ : 74.6 maize starch, 9.0 xylan, 9.0 pectin, 9.0 amylopectin, 9.0 arabinogalactan, 9.0 arabinoxylan, 9.0 xyloglucan, 31.5 Tween $80,43.7$ casein, 0.7 ox-bile, 43.7 bactopepton, $4.7 \mathrm{~K}_{2} \mathrm{HPO}_{4} .3 \mathrm{H}_{2} \mathrm{O}, 0.009 \mathrm{FeSO}_{4} .7 \mathrm{H}_{2} \mathrm{O}$, $8.4 \mathrm{NaCl}, 0.8 \mathrm{CaCl}_{2} .2 \mathrm{H}_{2} \mathrm{O}, 0.7 \mathrm{MgSO}_{4} .7 \mathrm{H}_{2} \mathrm{O}, 0.05$ bile, 0.02 haemin and 0.3 cysteine $\cdot \mathrm{HCl}$, plus $1.5 \mathrm{~mL}$ of a vitamin mixture containing (per litre): $1 \mathrm{mg}$ menadione, $0.5 \mathrm{mg}$ vitamin B12, $2 \mathrm{mg}$ D-biotin, $10 \mathrm{mg}$ pantothenate, $5 \mathrm{mg}$ p-aminobenzoic, $4 \mathrm{mg}$ thiamine and $5 \mathrm{mg}$ nicotinamide acid. The $\mathrm{pH}$ was adjusted to 5.9. Dialysis liquid contained (per litre): $2.5 \mathrm{~g}$ $\mathrm{K}_{2} \mathrm{HPO}_{4} .3 \mathrm{H}_{2} \mathrm{O}, 0.005 \mathrm{~g} \mathrm{FeSO}_{4} .7 \mathrm{H}_{2} \mathrm{O}, 4.5 \mathrm{~g} \mathrm{NaCl}, 0.45 \mathrm{~g} \mathrm{CaCl}_{2} .2 \mathrm{H}_{2} \mathrm{O}, 0.05 \mathrm{~g}$ bile, $0.5 \mathrm{~g}$ $\mathrm{MgSO}_{4} \cdot 7 \mathrm{H}_{2} \mathrm{O}$ and $0.4 \mathrm{~g}$ cysteine $\mathrm{HCl}$, plus $1 \mathrm{~mL}$ of the vitamin mixture. All medium components were purchased at Tritium Microbiology (Eindhoven, The Netherlands). The pig fecal inoculum was a standardized microbiota from growing pigs collected from the floor (48 pens with 6 pigs/pen, Hypor Libra x Hypor Maxter, Hendrix Genetics, Boxmeer, The Netherlands), but only fresh feces and from the top (not toughing the floor) was selected. Feces was pooled and mixed with dialysate as described before ${ }^{16}$.

In order to create a complete anaerobic environment, SLIM with $90 \mathrm{~mL}$ dialysate in each of the 4 individual units was flushed with gaseous nitrogen for at least 3 hours before incorporating the standardized microbiota. Thirty $\mathrm{mL}$ of the standardized microbiota was added to each SLIM-unit, making the total volume $120 \mathrm{~mL}$. Figure 1 shows the experimental set-up for fibre addition to SLIM. The microbiota was adapted to the model with SIEMP for 16 hours. During the adaptation phase, SIEMP was added into each SLIM-unit at a rate of $2.5 \mathrm{~mL} / \mathrm{h}$ through the feeding syringe. At the end of the adaptation period, a 2-hour starving 
period was performed, which was used to allow all the carbohydrates within SIEMP to be fermented by the microbiota. Afterwards, the fibre adjustment period (48 hours) was performed, in which the microbiota was allowed to adapt to the test products (CON, CELL, PECT1, PECT2 and ALK-treated RSM). During this stage, carbohydrates in SIEMP were replaced with 7.5 grams/day of (treated) RSM which were added continuously in the model at a rate of $2.5 \mathrm{~mL} / \mathrm{h}$. At the end of the $48 \mathrm{~h}$ adaptation period, a shot of 5 grams of the different RSMs was given to the system at time point 48 hours.

\section{In vivo fermentation in growing pigs by means of the mobile nylon bag technique}

The mobile nylon bag technique (MNBT) studies were carried out at the Animal Nutrition Group of Wageningen University \& Research in Wageningen, The Netherlands. Two pigs (TN 70, Topigs Norsvin) with initial body weight of $28 \pm 6.8 \mathrm{~kg}$ were fitted with a simple T-cannula at the distal ileum ${ }^{20}$ for the insertion of nylon bags. After surgery, the pigs were individually housed on tenderfeet floors with small openings. They were fed their diet as mash. All experimental procedures were approved by the local institution for animal welfare (IVD) of Wageningen University \& Research.

The MNBT studies included the nine feedstuffs which are described above (CON, 4 RSM substrates treated with carbohydrases or ALK before digestion, and 4 RSM substrates treated with carbohydrases or ALK after digestion). The procedures were slightly modified from previous research ${ }^{21}$. Samples of 0.3 to $0.5 \mathrm{~g}$ of each feedstuff were ground and filled into a nylon cloth (bag size: $25 \mathrm{~mm}$ X $40 \mathrm{~mm}$, pore size $48 \mu \mathrm{m}$, Sefar Nitex, Heiden Swiss, 03$37 / 24$ ), and afterwards sealed by means of a heat sealer. Eight bags per feedstuff were prepared, 4 bags for 2 pigs. The bags were inserted in the distal ileum through the cannula divided over 10 days; two bags at a time with two or three insertion moments at 15 mins intervals per day. Some bags were not collected and these replicates were repeated. The average collection time was 126.1 mins (range from 42.3 mins to 175.5 mins). In total, 64 bags were retrieved from feces and directly frozen at $-20{ }^{\circ} \mathrm{C}$ before transporting to the laboratory. Bags were subsequently cleaned from adherent feces using ultrapure water and thereafter immediately freeze-dried.

\section{Sample collection}

\section{in vitro SLIM}

Lumen samples from time point $48 \mathrm{~h}$ (just before the shot) were analyzed for constituent monosaccharide composition, molecular weight distribution, and oligosaccharide profiling. Samples from lumen and spent dialysate were collected at time point 48.5, 49, 50, 52, 54, 56, and $72 \mathrm{~h}$. They were snap-frozen in liquid nitrogen and stored until analyses. Lumen samples were used to analyze microbiota composition, constituent monosaccharide composition, molecular weight distribution, and oligosaccharide profiling, while both lumen and dialysis samples were analyzed for SCFA concentrations.

\section{MNBT study}

Samples from MNBT studies were pooled together according to treatment, and used to analyze constituent monosaccharide composition.

\section{S rRNA gene sequencing}


Microbial DNA extraction and sequencing of the V3-V4 region of the 16S rRNA gene were performed by BaseClear B.V. (Leiden, The Netherlands). Briefly, genomic DNA extraction from a single sample at each time point was performed using the Quick-DNA ${ }^{\mathrm{TM}} \mathrm{Fecal} /$ Soil Microbe Miniprep Kit (Zymo Research, California, US) according to the manufacturer's instructions. Barcoded amplicons from the V3-V4 region of $16 \mathrm{~S}$ rRNA genes were generated using a 2-step PCR. 10-25 ng genomic DNA was used as template for the first PCR with a total volume of $50 \mu \mathrm{L}$ using the $341 \mathrm{~F}$ (5'-CCTACGGGNGGCWGCAG-3') and the 785R (5'-GACTACHVGGGTATCTAATCC-3') primers 22 appended with Illumina adaptor sequences. PCR products were purified (QIAquick PCR Purification Kit, Qiagen, Venlo, The Netherlands) and the size of the PCR products were checked on a Fragment analyzer (Advanced Analytical, Ankeny, US) and quantified by fluorometric analysis. Purified PCR products were used for the 2nd PCR in combination with sample-specific barcoded primers (Nextera XT index kit, Illumina, California, US). Subsequently, PCR products were purified, checked on a Fragment analyzer and quantified, followed by multiplexing, clustering, and sequencing on an Illumina MiSeq with the paired-end (2x) $300 \mathrm{bp}$ protocol and indexing by Baseclear B.V. (Leiden, The Netherlands). The sequencing run was analyzed with the Illumina CASAVA pipeline (v1.8.3) with demultiplexing based on sample-specific barcodes. Raw sequencing data was submitted to the European Nucleotide Archive under the accession number: PRJEB36980.

\section{Bioinformatics analysis}

The demultiplexed raw sequences obtained from BaseClear were processed using QIIME2 pipeline ${ }^{23}$. In short, reads were imported and quality filtered and dereplicated with q2-dada2 ${ }^{24}$. Next, dada2 was performed with paired-end reads and truncation parameters were as follows: the first 10 base pairs were trimmed off and at position 280 base pairs the fragment was truncated in forward reads, and at position 240 base pairs for the reverse reads. The processed sequences were used for all the downstream analyses. Alpha-diversity (Shannon index) and beta diversity (weighted and unweighted UniFrac) were analyzed by the q2phylogeny plugin (https://github.com/qiime2/q2-diversity). All scripts used in the current analysis were deposited in Supplemental file 1: R_Markdown.html.

Random Forest. The Random Forest supervised machine learning algorithm was performed to predict treatments and timepoints from microbiome composition. The predictive models were built in R using the "caret" package. Specifically, samples were divided into training (more than $60 \%$ of the total samples) and test sets. Once the data were split, the function 'train' was used to fit the random forest model. Afterward, class labels on the test set were predicted by using the function 'predict', and compared to the real class labels. To interpret random forest results, proximity plots were produced in R. To understand more about the random forest model, the amplicon sequence variant (ASV) with the most influence in the random forest prediction was identified. All the analyses were done in $\mathrm{R}$ version 3.5.3 program, and the following packages were used: bioformat, yaml, Biostrings, phyloseq, Hmisc, qiime2R, vegan, ggplot2, tidyverse, caret, and randomForest.

Phylogenetic Investigation of Communities by Reconstruction of Unobserved States, PICRUSt2. The PICRUSt 2 software ${ }^{25}$ was used to predict microbial functional abundances based on marker gene sequences. KEGG database was used to predict the results.

\section{Chemical analyses}


Short-chain fatty acids. Samples from lumen and dialysate were analyzed by Brightlabs (Venlo, The Netherlands) for determination of concentrations of SCFA. Ion exclusion chromatography (IEC) was applied on an 883 Ion Chromatograph (IC; Metrohm, Switzerland), using a Transgenomic IC Sep ICE-ION-300 column $(30 \mathrm{~cm}$ length, $7.8 \mathrm{~mm}$ diameter and $7 \mu \mathrm{m}$ particles) and a MetroSep RP2 Guard. The mobile phase consists of 1.5 $\mathrm{mM}$ aqueous sulphuric acid. A column flow rate of $0.4 \mathrm{ml}^{*} \mathrm{~min}^{-1}$ was used. The temperature of the column was $65{ }^{\circ} \mathrm{C}$. The organic acids were detected using suppressed conductivity detection. Samples were centrifuged $(21,000 \mathrm{~g}, 10 \mathrm{~min})$ and the clear supernatant was filtered through a $0.45 \mu \mathrm{m}$ PFTE filter and diluted with mobile phase (for lumen 1:5, for dialysate 1:2). Ten microliters were loaded on the column by an autosampler 730 (Metrohm). Molecules were eluted according to their pKa.

Constituent monosaccharide composition. The constituent monosaccharide content and composition were determined using a prehydrolysis step with $72 \%(\mathrm{w} / \mathrm{w} \%)$ sulfuric acid at $30{ }^{\circ} \mathrm{C}$ for $1 \mathrm{~h}$ followed by hydrolysis with $1 \mathrm{M}$ sulfuric acid at $100{ }^{\circ} \mathrm{C}$ for $3 \mathrm{~h}$. The monosaccharides formed upon hydrolysis were derivatized to alditol acetates and analyzed by gas chromatography using inositol as internal standard ${ }^{26}$. The colorimetric $m$ hydroxydiphenyl assay was used to determine the total uronic acid content ${ }^{27}$.

Molecular weight distribution. Fermentation digests (corresponding to $2 \mathrm{~mL}$ lumen samples) or dry raw materials, which were dissolved in ultrapure water, were centrifuged (10 min, $18,000 \mathrm{~g}, 24{ }^{\circ} \mathrm{C}$ ) to obtain the soluble fraction, which was analyzed for molecular weight distribution using high-performance size exclusion chromatograph (HPSEC) on an Ultimate 3000 HPLC (Dionex, Sunnyvale, CA, USA). Three SK-Gel columns in series (4000-3000-2500 Super AW; 150 X 6 mm) were used for the analysis. All columns were from Tosoh Bioscience (Tokyo, Japan). Pullulan molecular-mass standards (Polymer Laboratories, Palo Alto, CA, USA) were used for calibration ${ }^{28}$.

Oligosaccharide Profiling. High-performance anion exchange chromatograph (HPAEC) was performed on an ICS5000 system (Dionex), equipped with a Dionex CarboPac PA-1 colomn ( 2 X $250 \mathrm{~mm}$ ) in combination with a CarboPac PA-1 guard column ( 2 X 250). The flow rate was $0.3 \mathrm{~mL} / \mathrm{min}$ with an eluent profile starting with $0.02 \mathrm{M} \mathrm{NaOH}$ until $13 \mathrm{~min}$, then increasing to $0.1 \mathrm{M} \mathrm{NaOH}$ until $15 \mathrm{~min}$, followed by a linear gradient of $0-500 \mathrm{mM}$ $\mathrm{NaOAc}$ in $0.1 \mathrm{M} \mathrm{NaOH}$ until $45 \mathrm{~min}$, followed by a gradient to $1 \mathrm{M} \mathrm{NaOAc}$ in $0.1 \mathrm{M} \mathrm{NaOH}$ in $1 \mathrm{~min}$ and $7 \mathrm{~min}$ at $1 \mathrm{M} \mathrm{NaOAc}$ in $0.1 \mathrm{M} \mathrm{NaOH}$. After this, the column was equilibrated with $0.1 \mathrm{M} \mathrm{NaOH}$ for $3 \mathrm{~min}$ and $0.02 \mathrm{M} \mathrm{NaOH}$ for $20 \mathrm{~min}$. An ICS5000ED (Dionex) Pulsed Amperometric Detector and Chromeleon software version 7 were used. Oligomers of cellulose (DP 2-DP 6) were used as standards to identify cellulose oligomers in the elution profile.

\section{Statistics}

Kruskal-Wallis Rank Sum Test (one-way ANOVA on ranks) was applied to compare alpha diversities (Shannon index and Faith's PD) among different RSM treatments, and Wilcoxon Rank Sum Test was used for pairwise comparison in R version 3.5.3 (https://www.rproject.org/). Bonferroni adjustments were used to correct P-values for multiple comparisons. Permutational multivariate analysis of variance (PERMANOVA) was performed to test the significance of beta diversity (weighted and unweighted UniFrac) between non-processed and processed RSM in QIIME2. The results were visualized in R. 
The ASV table (feature table of QIIME2) was normalized and filtered in R and statistical analysis was performed in STAMP ${ }^{29}$. The table was normalized via division by the sum of sequences in a given sample and multiplied by the minimum sum across all samples. Relative abundances were filtered as following: values below a relative abundance threshold of $0.01 \%$ were not taken into account; taxa with a median relative abundance $<1 \%$ in all groups were not considered for statistical analysis. White's non-parametric t-test was applied to compare between CON group and treatments. $P$-values were corrected using the Benjamini-Hochberg method. A $q$-value (corrected $P$-value) $<0.05$ was considered significant.

Pearson correlations between continuous meta-variables and taxonomic variables were calculated and visualized in $\mathrm{R}$ ( $\mathrm{R}$ version 3.5.3). Parameters were set as following: Missing values for meta-variables were handled as NO imputation (replacing missing data with substituted); zeros were kept for the calculation of correlation; a minimum number of $0.1 \%$ was considered for calculation; a minimum of 4 paired observations were required for calculation of correlations.

T-tests were conducted to compare SCFA production between CON and the treated RSM substrates in the built-in $\mathrm{R}$ package ( $\mathrm{R}$ version 3.5.3). 


\section{Results}

\section{Description and characteristics of untreated and processed RSM}

Table 1 shows the constituent monosaccharide composition of (processed) RSM. The carbohydrate content of $\mathrm{CON}$ is $62 \% \mathrm{w} / \mathrm{w}$. Dominant sugars are glucose (Glc, $31 \mathrm{~mol} \%$ ), uronic acid (UA, $19 \mathrm{~mol} \%$ ), arabinose (Ara, $5 \mathrm{~mol} \%$ ), and galactose (Gal, $11 \mathrm{~mol} \%$ ). CON contained $56 \%$ pectin (defined as: rhamnose + arabinose + galactose + uronic acid) and $44 \%$ (hemi)cellulose (xylose + mannose + fucose + glucose). The values of pectin and (hemi)cellulose for ALK_A, ALK_B, PECT1_A, PECT1_B, PECT2_A, PECT2_B, CELL_A, and CELL_B were $55 \%$ and $45 \%, 52 \%$ and $48 \%, 54 \%$ and $46 \%, 50 \%$ and $50 \%$, $56 \%$ and $44 \%, 51 \%$ and $49 \%, 57 \%$ and $43 \%$, and $60 \%$ and $40 \%$, respectively. Relative pectin contents decreased in ALK, PECT1, and PECT2 treatment, while they increased in CELL treatment, compared to CON. ALK, PECT1, and PECT2 treatment increased (hemi)cellulose values, compared to CON. Predigesting before or after processing RSM had little effect on the monosaccharide constitution.

Table 1. Constituent monosaccharide composition of processed RSM

\begin{tabular}{|c|c|c|c|c|c|c|c|c|c|}
\hline & \multicolumn{8}{|c|}{$(\mathrm{mol} \%)$} & \multirow{2}{*}{$\begin{array}{r}(\mathrm{w} / \mathrm{w} \%) \\
\text { Total }\end{array}$} \\
\hline & Rha & Fuc & Ara & Xyl & Man & Gal & Glc & UA & \\
\hline $\mathrm{CON}$ & 1 & 1 & 25 & 10 & 2 & 11 & 31 & 19 & 62 \\
\hline ALK_A & 1 & 1 & 23 & 9 & 3 & 11 & 35 & 17 & 58 \\
\hline ALK_B & 1 & 1 & 26 & 9 & 2 & 8 & 32 & 21 & 52 \\
\hline PECT̄1_A & 1 & 1 & 26 & 9 & 2 & 14 & 37 & 9 & 61 \\
\hline PECT1_B & 1 & 1 & 22 & 8 & 2 & 10 & 34 & 21 & 53 \\
\hline PECT2_A & 1 & 1 & 16 & 8 & 3 & 11 & 37 & 22 & 63 \\
\hline PECT2_B & 1 & 1 & 23 & 9 & 3 & 11 & 31 & 21 & 57 \\
\hline CELL_A & 1 & 1 & 24 & 9 & 2 & 11 & 28 & 23 & 63 \\
\hline CELL_B & 1 & 1 & 24 & 9 & 3 & 10 & 30 & 23 & 56 \\
\hline
\end{tabular}

Rha, rhamnose; Fuc, fucose; Ara, arabinose; Xyl, xylose; Man, mannose; Gal, galactose, Glc, glucose; UA, Uronic acid. _A, RSM was treated after predigesting, _B, RSM was treated before predigesting.

\section{Considerable changes occurred in the microbiota fed with ALK- and CELL-processed} RSM after a shot of 5 grams of test products.

Changes in the gut microbiota in response to a shot of 5 grams of the different treated RSM substrates were determined. When data from all of the time points were pooled, Shannon index of ALK and CELL were significant lower than CON, while there were no significantly differences for PECT1 and PECT2 compared to CON (Figure 2). Within the same group, different time points did not appear significantly different in term of Shannon index (data not shown). Phylogeny based UniFrac distance matrix measurements were then used to compare the $\beta$-diversity of the microbial communities between microbiota fed with non-processed and processed RSM. Unweighted UniFrac, which is clustering data based on presence or absence of ASV, clustered the non-processed and processed RSMs samples separately $(P<$ 0.001). There was no clear separation between CON and PECT1, and between CON and PECT2 in terms of weighted UniFrac metrics $(P>0.05)$, which also considers the relative abundance of the ASV, while CON was significantly different with ALK and CELL $(P<$ 0.001) (Figure 3, and S1). Both weighted and unweighted UniFrac revealed that bacterial community structure of CON were more similar to PECT1 and PECT2 than those of ALK and CELL, while microbial community compositions of PECT1 and PECT2 were similar to 
each other $\left(P_{\text {weighted UniFrac }}=0.131, P_{\text {unweighted Unifrac }}=0.078\right)($ Supplemental file 2: Figure S1 $)$.

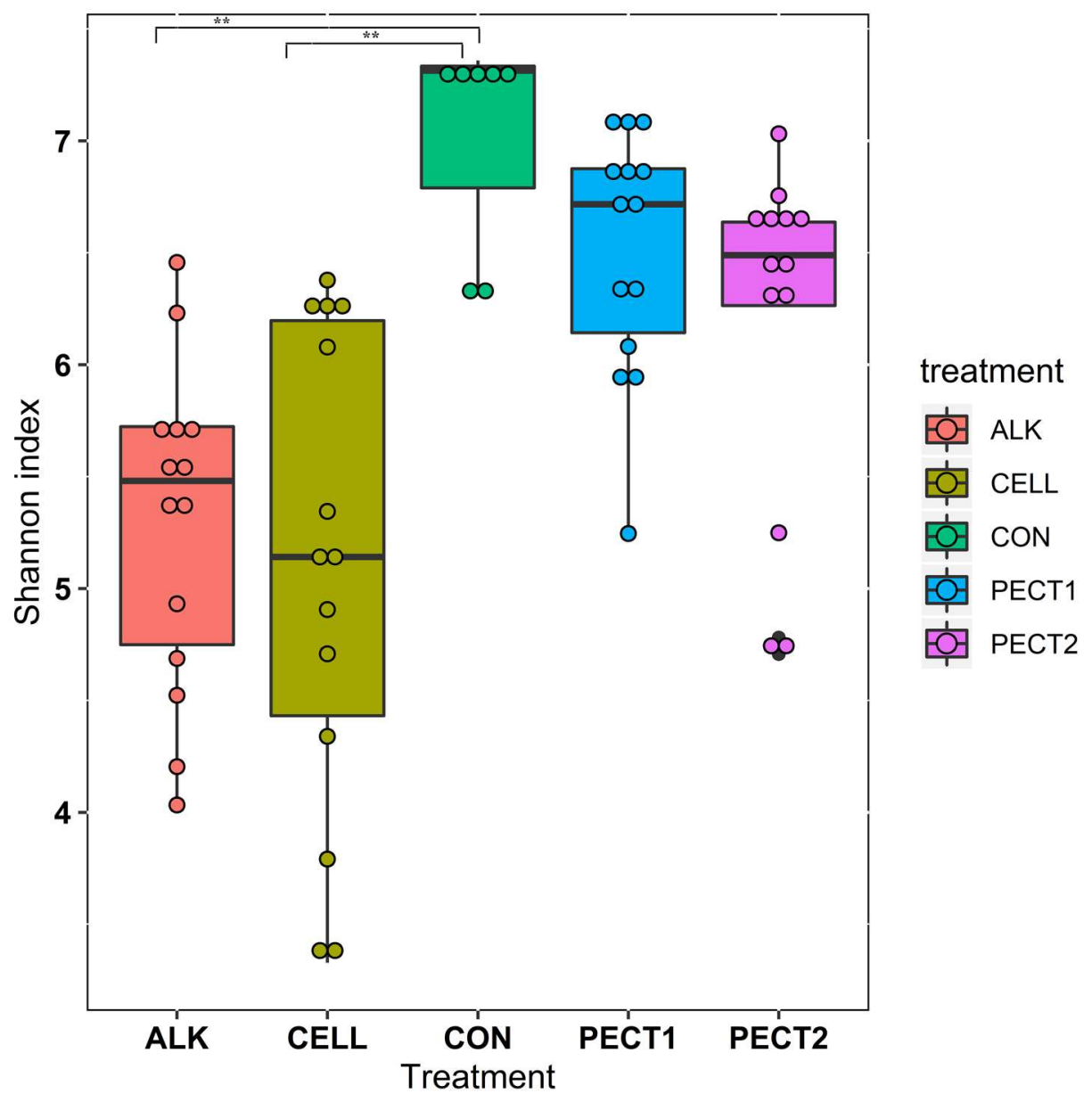

Figure 2. Community diversity represented by Shannon index at ASV level for samples from each treatment. Shannon index was calculated based on the average of ten iterations at equal sampling-depth 7139 for each sample. Each bar represents the samples from the microbiota fed with non-processed RSM (CON) and RSM processed by Accellerase 1000 (CELL), Pectinex Ultra SP (PECT1), Multifect Pectinase (PECT2), or 6M NaOH (ALK). 


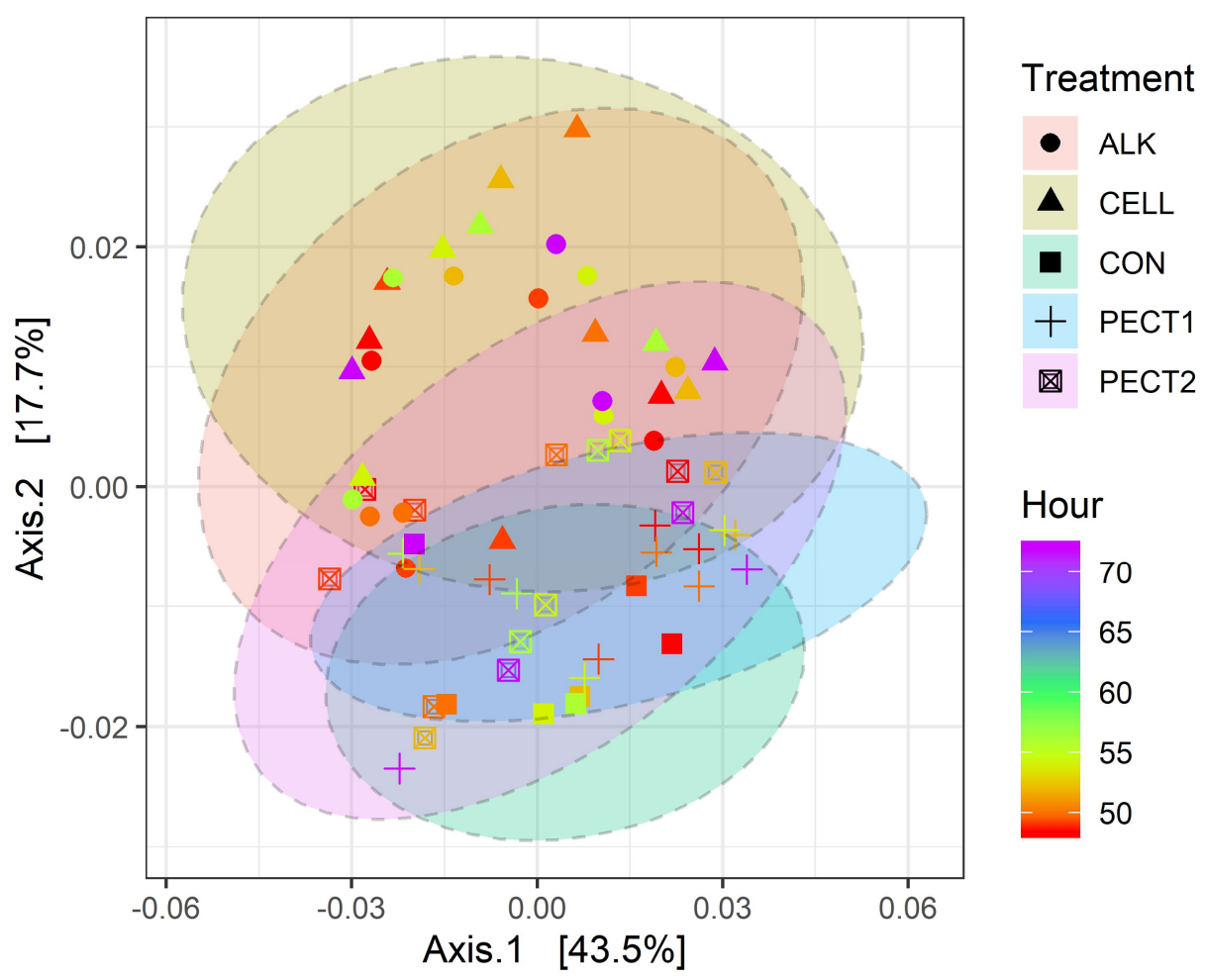

Figure 3. Principle coordinate analysis ( $\mathrm{PCoA})$ plot generated based on the calculated distances in the unweighted matrix. Samples were grouped by shape and colour in terms of treatment and time point, respectively: non-processed RSM (CON), square; RSM processed by $6 \mathrm{M} \mathrm{NaOH}$ (ALK), sphere; RSM processed by Pectinex Ultra SP (PECT1), plus; RSM processed by Multifect Pectinase (PECT2), square with cross inside; RSM processed by Accellerase 1000 (CELL), triangle; a red-green-purple scale was used to indicate the fermentation time (red and purple depict start and end of the fermentation period).

Alpha-diversity (Shannon index) and $\beta$-diversity (weighted and unweighted UniFrac) showed that there were no significant differences between microbiota fed with processed RSM before or after dialysis (data not shown). This indicated that predigesting before or after processing RSM by ALK or enzymes, did not result in different microbiome compositions. Furthermore, predigesting before or after processing RSM also had little effect on their monosaccharide constitution as described above. Therefore, they were treated as duplicates in the (following) microbial relative abundance analysis.

We next compared microbial relative abundance of CON with those of the microbiotas fed with the different processed RSMs to identify significantly different bacterial taxa. Data from all time points were grouped. No significant differences were detected at the phylum level when comparing CON to the other groups (ALK, PECT1, PECT2 and CELL) (data not shown). At the genus level, when compared to CON, ALK treatment resulted in significantly 
increased relative abundance of Olsenella $(P=0.017)$, Runimicoccus gauvreauii group $(P=$ 0.019), Eubacterium nodatum group $(P<0.001)$, Megasphaera $(P<0.001)$, Bifidobacterium $(P<0.001)$, Acidaminococcus $(P<0.001)$, and Acetitomaculum $(P<0.001)$, which were from the phyla Actinobacteria and Firmicutes. In addition ALK treatment significantly decreased the relative abundance of Ruminococcaceae UCG-002 $(P<0.001)$, Christensenellaceae $R-7$ group $(P<0.001)$, Enterobacteriaceae unknown group $(P<0.001)$, $p$-2534-18B5 gut group from the order Bacteroidales $(P<0.001)$, Citrobacter $(P<0.001)$, Prevotella $9(P=0.004)$, Rikenellaceae $R C 9$ gut group $(P=0.006)$, Desulfovibrio $(P=$ $0.007)$, Prevotellaceae NK3B31 group $(P=0.030)$, and Lachnoclostridium $(P=0.031)$, which were from the phyla Bacteroidetes, Firmicutes and Proteobacteria (Figure 4 and Supplemental file 2: Figure S2).
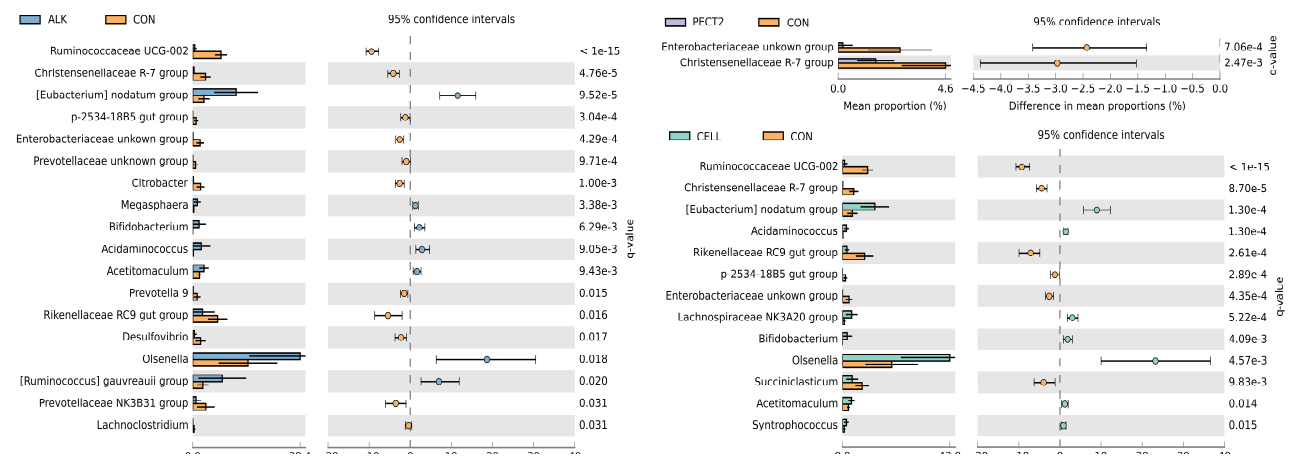

Figure 4. Significantly different microbial genera in relative abundance in the different treatments compared to CON. White's non-parametric T-test was applied to compare between $\mathrm{CON}$ group and treatments. $P$-values were corrected using the Benjamini-Hochberg method ( $q$-values). The shown mean relative abundance percentages of the taxa were calculated using all samples taken over time within each treatment.

PECT2 treatment significantly decreased the relative frequencies of Enterobacteriaceae unknown group $(P<0.001)$ and Christensenellaceae $R-7$ group $(P<0.001)$ when compared with CON group (Figure 4). There was no significant difference at genus level between CON group and PECT1 treatment group.

Relative abundance of Olsenella $(P<0.001)$, Eubacterium nodatum group $(P<0.001)$, Acidaminococcus $(P<0.001)$, Lachnospiraceae NK3A20 group $(P<0.001)$, Bifidobacterium $(P<0.001)$, Acetitomaculum $(P=0.014)$, and Syntrophococcus $(P=0.016)$, from the phyla Actinobacteria and Firmicutes, significantly increased in microbiota fed CELL-processed RSM. Moreover, CELL treatment decreased the relative abundance of Ruminococcaceae UCG-002, Christensenellaceae $R-7$ group, $p$-2534-18B5 gut group from the order Bacteroidales, Rikenellaceae RC9 gut group, and Succiniclasticum, which were from Firmicutes and Bacteroidetes (Figure 4 and S2).

ALK and CELL significantly increased the microbial functional abundances related to fibre degradation and SCFA production compared to CON.

PICRUST2 was performed to the 16S rRNA gene data to predict metagenomic functional profiles. Compared with CON, 111 features were significantly different in ALK, 108 features 
in CELL, 2 features in PECT1 and 1 feature in PECT2 (Supplemental file 2: Figure S3). As the current study focused on fibre degradation, only carbohydrate metabolism related microbial functional features are summarized. The relative abundances of carbohydrate digestion and absorption $(P=0.047)$, galactose metabolism $(P=0.008)$, glycolysis $(P=$ $0.001)$, pentose phosphate pathway $(P=0.004)$, propanoate metabolism $(P<0.001)$, and pyruvate metabolism $(P<0.001)$ were predicted to be significantly higher upon ALK feeding, while glycan biosynthesis and metabolism $(P=0.005)$, and lipopolysaccharide biosynthesis $(P=0.005)$ were significant higher in CON (Figure 5A). After the microbiota was fed with CELL, microbial functional abundance of carbohydrate digestion and absorption $(P=0.019)$, energy metabolism $(P=0.018)$, fructose mannose metabolism $(P=0.020)$, galactose metabolism $(P=0.010)$, glycerolipid metabolism $(P<0.001)$, glycolysis $(P<0.001)$, pentose phosphate pathway $(P=0.004)$ and pyruvate metabolism $(P=0.001)$ significantly increased (Figure 5B). There were no significant changes in carbohydrate metabolism related microbial abundance upon feeding PECT1 or PECT2 when compared to CON.

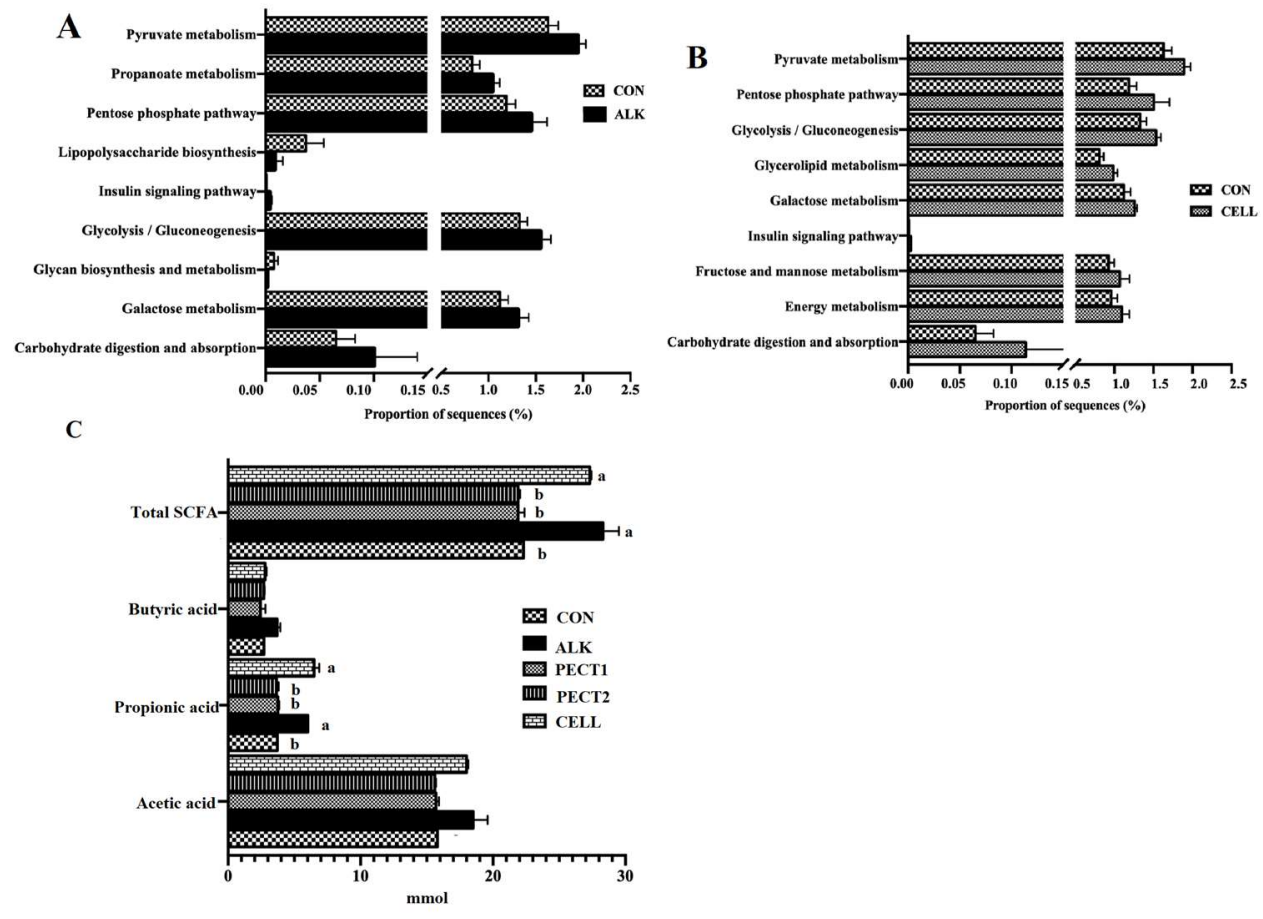

Figure 5. Significantly different metagenomic functions in relative abundance in the treatments ALK (A) and CELL (B) compared to CON. Difference of short-chain fatty acid production (C) during fermentation of ALK, PECT1, PECT2, and CELL compared to CON.

Cumulative short-chain fatty acid production by the microbiota fed with CON was compared with those by the microbiotas fed with ALK, PECT1, PECT2 or CELL. The amount of propionic acid $\left(P_{\mathrm{ALK}}=0.010, P_{\mathrm{CELL}}=0.006\right)$ and the total SCFA $\left(P_{\mathrm{ALK}}=0.008, P_{\mathrm{CELL}}=\right.$ 0.015) in ALK and CELL were significant higher than those in CON, while there were no significant differences in SCFA production in PECT1 and PECT2 compared with CON 
(Figure 5C).

\section{Random Forest revealed a RSM degradation pattern in a porcine gut microbiota.}

The supervised machine learning technique Random Forest was applied to predict fermentation time. Every possible time interval was used \{e.g. $(48,49](49,72],(48,50](50$, $72],(48,52](52,72],(48,54](54,72]$, and $(48,56](56,72]\}$, but only the time interval, $(48$, 52] $(52,72]$, performed well at the prediction task (Supplemental file 3: Table S1). The Random Forest proximity plot shows that samples from time point 48.5 to 52 were clustered (Figure 6A). This indicated that microbiota composition considerably changed only after incubation for 4 hours after a shot of 5 grams of treated RSM, which may have occurred because the nutrient composition in the lumen significantly changed. To further understand the Random Forest model, the ASV with the most influence in the Random Forest prediction was identified, which turned out to be a genus in the family Veillonellaceae: Megasphaera (Figure 6B). Megasphaera was also significantly higher in ALK when tested with the Kruskal-Wallis non-parametric test (Figure 4).
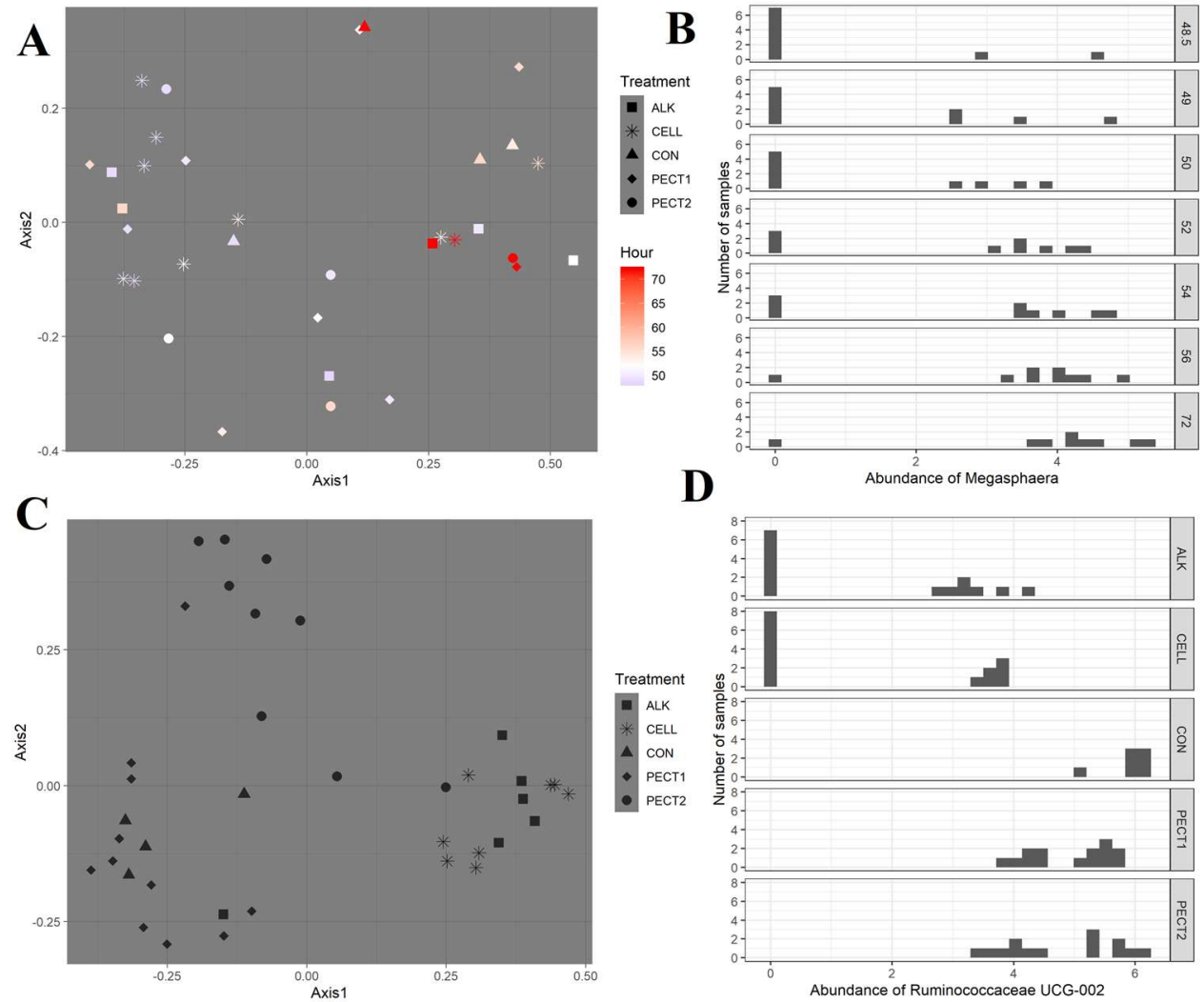

Figure 6. Random Forest proximity plots of time points (A) and treatments (C), and the ASV with the most influence in the random forest prediction (B for time points, $D$ for treatments). To generate this representation, a distance was calculated between samples based on how frequently samples occur in the same tree partition in the random forest's bootstrapping 
procedure. If a pair of samples frequently occured in the same partition, the pair was assigned a low distance. The resulting distances are then input to the PCA.

Interestingly, the effect on the microbiota composition of the different processing methods on RSM was also predicted by Random Forest. Table S2 (Supplemental file 2) shows that Random Forest performed well at this prediction task, and the Random Forest proximity plot demonstrates that microbiota fed with CON had less difference with PECT1 and PECT2, while there was a larger distance with ALK and CELL (Figure 6C). The microorganisms with the most influence in classifying the different processing methods was in family Ruminococcaceae: Ruminococcaceae UCG-002 (Figure 6D).

\section{HPSEC elution profiles showed almost complete degradation of soluble high molecular weight polysaccharides at time point $52 \mathrm{~h}$.}

Molecular weight distributions of soluble fibres from RSM with different processing methods are shown in Figure 7. For ALK, PECT1, PECT2 and CELL fermentation, an increase in the amount of soluble materials corresponding to high molecular weight $\left(\mathrm{M}_{\mathrm{w}}\right)$ was seen $2,1,1$, and $1 \mathrm{~h}$ after the shot (at time point 50 or $49 \mathrm{~h}$ ), respectively. While for $\mathrm{CON}$, the increase in soluble materials with high $\mathrm{Mw}$, was seen already after $0.5 \mathrm{~h}$ of incubation (at time point $48.5 \mathrm{~h}$ ). Although high $\mathrm{M}_{\mathrm{w}}$ material was observed before the shot of 5 grams RSM (at time point 48), indicating that the starvation period did not lead to complete fermentation of the fibres, as observed before ${ }^{30}$, they were degraded rapidly after $0.5 \mathrm{~h}$ (at time point $48.5 \mathrm{~h}$ ), since the peaks of $48.5 \mathrm{~h}$ were lower than those of $48 \mathrm{~h}$, which can be seen in the HPSEC profiles of ALK, PECT1, PECT2 and CELL. From $4 \mathrm{~h}$ onward (at time point $52 \mathrm{~h}$ ), an almost complete disappearance in the high $\mathrm{M}_{\mathrm{w}}$ fraction occurred, indicating degradation and/or utilization of all high $\mathrm{M}_{\mathrm{w}}$ polysaccharides.
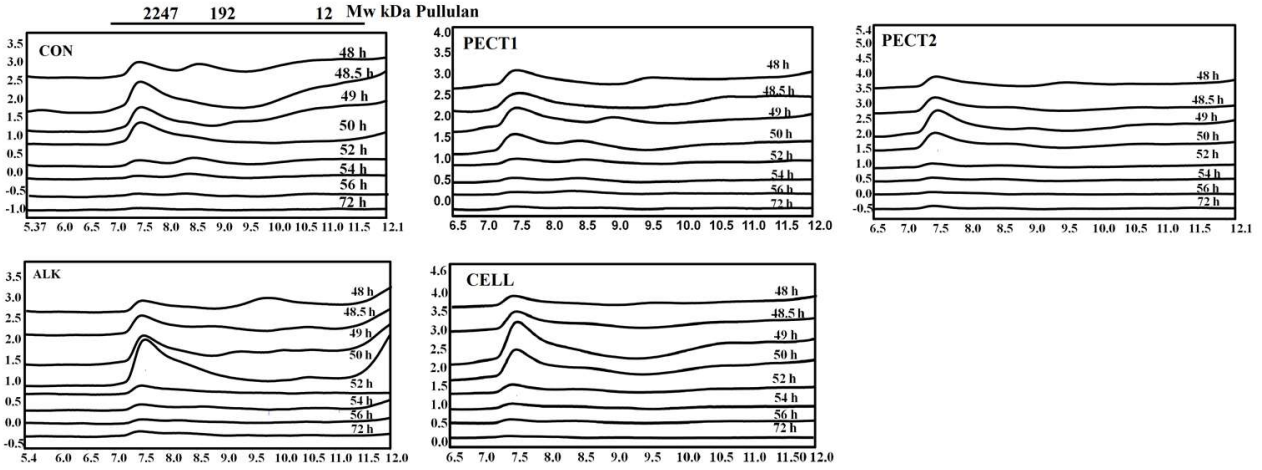

Figure 7. HPSEC elution patterns of soluble fraction of fermentation digests during fermentation of CON (A), ALK (B), PECT1 (C), PECT2 (D), and CELL (E). Pullulan was used as a calibrant.

Compared to the highest peak of the elution profile of CON $(48.5 \mathrm{~h})$, the highest peaks of the elution profile of ALK and CELL ( $50 \mathrm{~h}$ and $49 \mathrm{~h}$, respectively) showed a higher amount of high $\mathrm{M}_{\mathrm{w}}$ material, while those of the elution profile of PECT1 and PECT2 were 
approximately equal to CON (Figure 7). This indicated that more materials were solubilized under the conditions when ALK and CELL were fed, than with the other substrates.

HPAEC elution profiles indicated large amount of soluble oligo-celluloses formed during ALK and CELL fermentation.

To determine oligomers formed and utilized during fermentation of processed versus nonprocessed RSM, soluble fractions from fermentation digests were analyzed using HPAEC (Figure 8). For ALK and CELL, a few peaks can be identified as oligomers of cellulose on the basis of standards of cellulose oligomers as indicated in Figure 8 (cellobiose, cellotriose, cellotetraose, cellopentaose, cellohexaose). However, most of the peaks in ALK and CELL fermentation samples were not identifiable on the basis of the cellulodextrin standard. These oligomers are only present upon ALK and CELL feeding, and were not seen with PECT1, PECT2 and CON. The oligomers were increased to highest amounts $0.5 \mathrm{~h}$ after addition of the $5 \mathrm{~g}$ shot (time point $48.5 \mathrm{~h}$ ), and decreased to be almost completely fermented after $8 \mathrm{~h}$ (time point $56 \mathrm{~h}$ ).
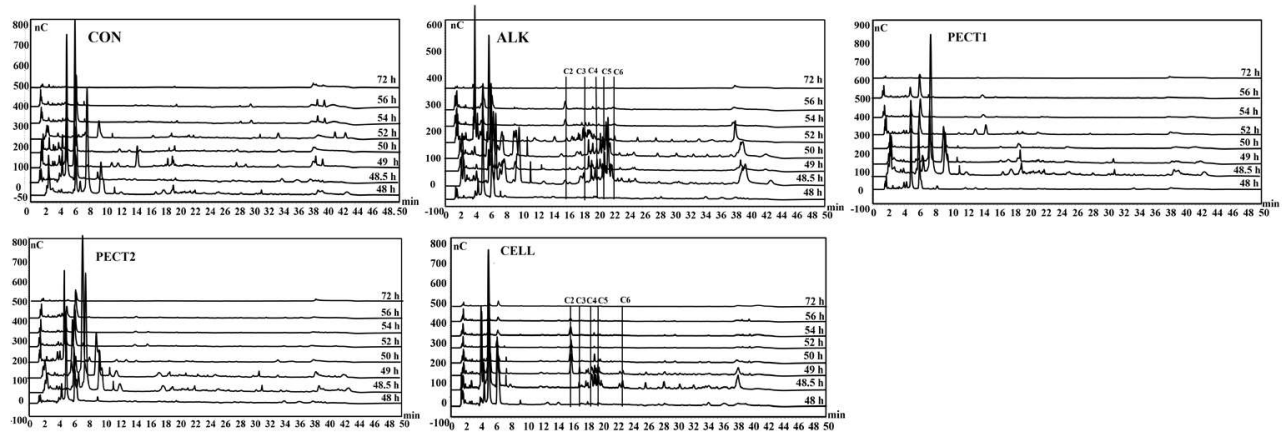

Figure 8, HPAEC elution profile of fermentation digests during fermentation of CON (A), ALK (B), PECT1 (C), PECT2 (D), and CELL (E). C2: D- $(+)$ cellobiose; C3: 1,4- $\beta$-Dcellotriose; C4: 1,4- $\beta$-D-cellotetrose; C5: 1,4- $\beta$-D-cellopentose; C6: $1,4-\beta$-D-cellohexose.

No pectin oligomers were observed in both enzymatic and chemical treatment groups.

\section{Constituent monosaccharide composition of fermentation samples showed that less residual carbohydrates remained in ALK and CELL compared to CON.}

Direct utilization of polysaccharides during RSM fermentation can be revealed from the decrease in the carbohydrate content. Figure 9 shows the utilization of the main monosaccharides in RSM, which are arabinose, galactose, glucose, and uronic acid. The amount of main monosaccharides was lower after $24 \mathrm{~h}$ fermentation (at time point 72 ) for ALK and CELL compared to CON. The utilization of the main monosaccharides plateaued after time point 52, except for glucose, which was continuously utilized until time point 72 . The utilization of arabinose and galactose were faster in ALK and PECT1, compared to the other treatments. 

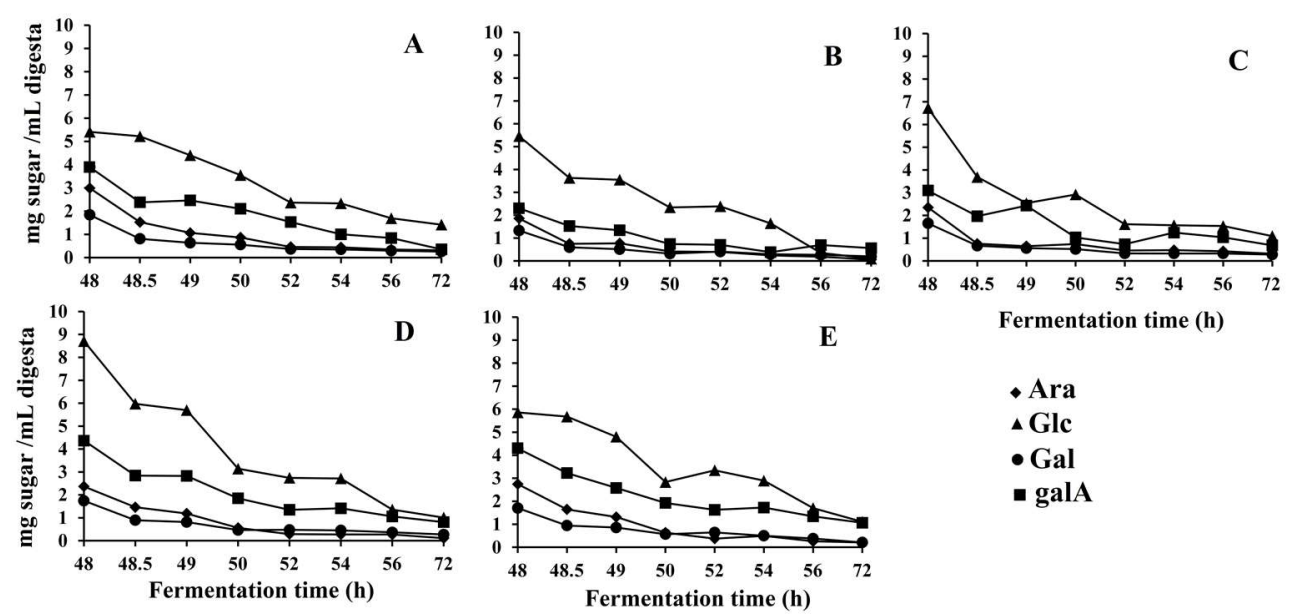

Fermentation time (h)

- Ara

$\triangle$ Glc

- Gal

- galA

Figure 9. Utilization of arabinose (Ara), galactose (Gal), glucose (Glc), and uronic acid (UA) present in CON (A), ALK (B), PECT1 (C), PECT2 (D), and CELL (E) during in vitro fermentation. Values presented are means of duplicates measurements.

\section{Correlation between microbiota abundance and SCFA production and monosaccharide composition.}

Correlations between the relative abundances at genus level and SCFA production and monosaccharide composition $(\mathrm{mg} / \mathrm{mL}$ sugar left) at each time point were analyzed (Figure 10). Bifidobacterium, [Eubacterium] nodatum group, and Acidaminococcus had significant negative correlations with acetic and butyric acid, while Prevotella 7 and Megasphaera had significant positive correlation with acetic, propionic and butyric acid. Butyric acid significantly positively correlated with Prevotellaceae NK3B31 group and Desulfovibrio. 


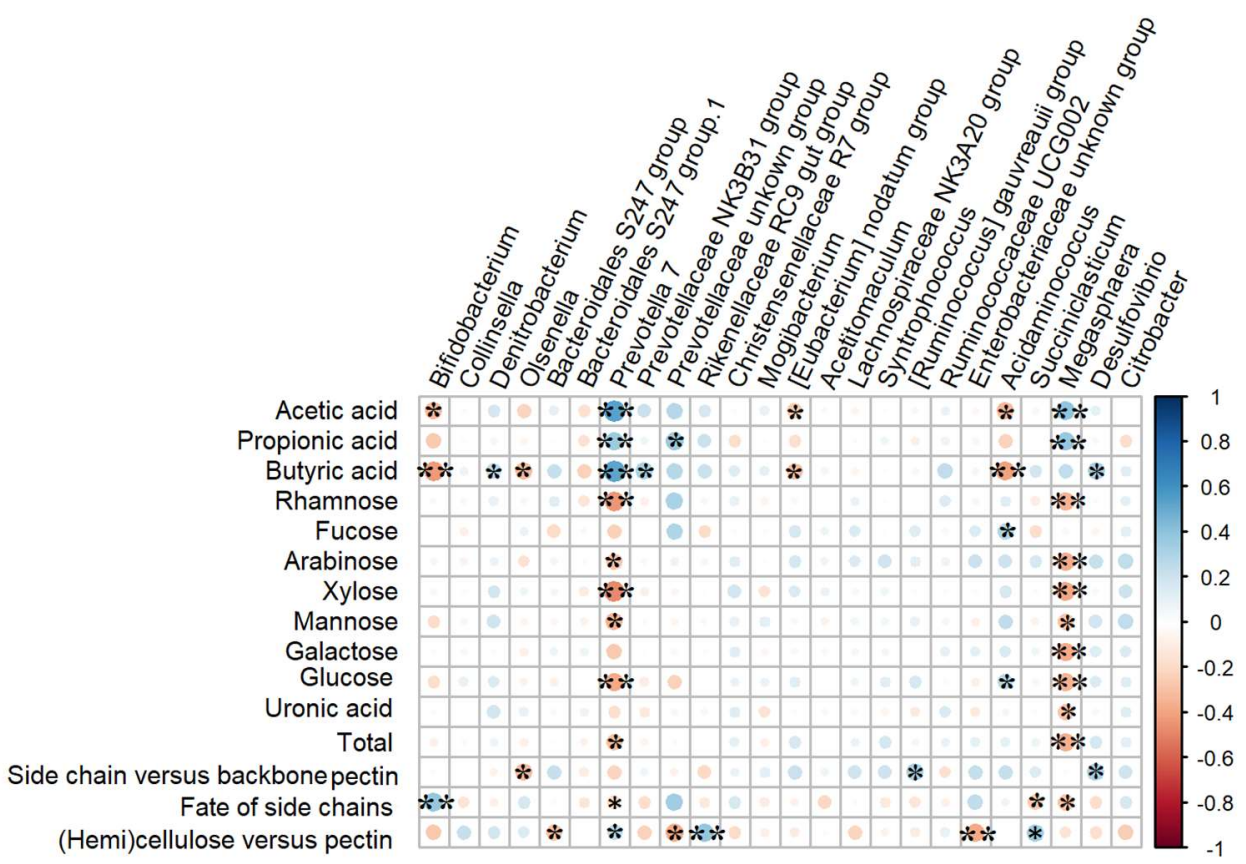

Figure 10. Correlation between core bacterial genera and SCFA production and molar percentage of monosaccharides. Statistical significance was determined for all pairwise comparisons using Spearman's method.. The relative abundances of ASVs were significantly negatively correlated with monosaccharides $(\mathrm{mg} / \mathrm{mL}$ sugar left in the lumen), which indicated the more bacterial abundance, the more utilization of these monosaccharides. For instance, Prevotella 7 was significantly negatively correlated with Rha, which means that more Rha was utilized when the relative abundance of Prevotella 7 increased. Side chain versus backbone pectin $=(\mathrm{Ara}+\mathrm{Gal}):(\mathrm{galA}+\mathrm{Rha})$, Fate of side chains $=$ Ara $: \mathrm{Gal}$, $($ Hemi $)$ cellulose versus pectin $=(\mathrm{Xyl}+\mathrm{Glc}+\mathrm{Man}):(\mathrm{galA}+\mathrm{Gal}+\mathrm{Ara}+\mathrm{Rha}) . \mathrm{Rha}$, rhamnose; Fuc, fucose; Ara, arabinose; Xyl, xylose, Man, mannose, Gal, galactose, Glc, glucose; galA, assumed to be equal to uronic acid; Total, the total sugar left in the lumen * $P<0.05$; ** $P<0.01$. Size of the circles means correlation values, which bigger sizes meaning higher correlation values. Blue circles represent positive correlations, whereas red circles represent negative correlations.

Rha, Ara, Xyl, Man, Glu, and total monosaccharides (Total) had significant negative correlations with Prevotella 7 and Megasphaera, and Gal and UA also had significant negative correlations with Megasphaera. Olsenella was significantly negatively correlated with 'Side chain versus backbone pectin' $[=(\mathrm{Ara}+\mathrm{Gal}):(\mathrm{galA}+\mathrm{Rha})]$, while [Ruminococcus $]$ gauvreauii group and Desulfovibrio had significant positive correlation with this. Bifidobacterium was significantly positively correlated with 'Fate of side chains' [Ara : Gal], whereas Succiniclasticum and Megasphaera had significant negative correlation with this. Bacteroidales S24-7 group and unknown genera from family Prevotellaceae and Enterobacteriaceae had significant negative correlation with '(Hemi)cellulose versus pectin' $[(\mathrm{Xyl}+\mathrm{Glc}+\mathrm{Man}):(\mathrm{galA}+\mathrm{Gal}+\mathrm{Ara}+\mathrm{Rha})]$, while Prevotella 7 and Rikenellaceae RC9 gut group were significantly positively correlated with this. 
MNBT validated that more fibres were degraded in case of ALK and CELL treatment, compared to PECT1 and PECT2.

Utilization of cell wall polysaccharides upon RSM fermentation assessed using the ileal cannulated growing pigs can be revealed from the decrease in the total carbohydrate content as well as from the constituent monosaccharides of the material in the collected bags after transit through the pigs (Table 2). After the nine substrates (in the nylon bags) passed through the ileal cannulated growing pigs, there were $31 \%(\mathrm{CON}), 11$ and $22 \%$ ALK_A and ALK_B, 30 and $31 \%$ PECT1_A and_B, 30 and $30 \%$ PECT2_A and B, and 19 and $22 \%$ CELL_A and $B$ carbohydrates left in the residues, respectively (calculated from the sugar composition and the recovery of DM). The constituent monosaccharides of non-processed and processed RSM were similar to each other (Table 1), while mol percentages of Ara, Glc and Gal were shifted in ALK and CELL after fermentation in sacco (Table 2). The percentages of Ara and Gal were lower in ALK and CELL after fermentation, where the mol percentages of Ara decreased from $23 \%$ to $9 \%$ for ALK_A, $26 \%$ to $5 \%$ for ALK_B, $24 \%$ to $5 \%$ for CELL_A and from $24 \%$ to $10 \%$ for CELL_B (Table 1 and Table 2 ). Molar percentages of Gal were diminished from $11 \%$ to $6 \%$ in ALK_A, from $8 \%$ to $4 \%$ in ALK B, $11 \%$ to $6 \%$ in CELL_A, and from $10 \%$ to $7 \%$ in CELL_B. Glc was increased from $35 \%$ to $37 \%$ in ALK_A, from $32 \%$ to $68 \%$ in ALK_B, from $28 \%$ to $50 \%$ in CELL_A, and from $30 \%$ to $37 \%$ in CELL_B. On top of this change in mol percentages, the recovery of DM was also reduced by at least $69 \%$, as described above, indicating e.g. that for ALK_A (11\% DM recovery) Ara decreased by approximately 25-fold (Supplemental file 2: Table S3).

Table 2. Constituent monosaccharide composition of residues obtained from in sacco fermentation of CON, ALK, PECT1, PECT2 and CELL in ileal cannulated pigs.

\begin{tabular}{|c|c|c|c|c|c|c|c|c|c|c|}
\hline & \multicolumn{8}{|c|}{$(\mathrm{mol} \%)$} & \multirow[b]{2}{*}{ w/w $\%$ Total } & \multirow{2}{*}{$\begin{array}{c}\text { (w/w \%) } \\
\text { Recovery } \\
\text { of DM }\end{array}$} \\
\hline & Rha & Fuc & Ara & Xyl & Man & Gal & Glc & UA & & \\
\hline $\mathrm{CON}$ & 2 & 1 & 17 & 9 & 2 & 10 & 40 & 20 & 87 & 36 \\
\hline ALK_A & 2 & 1 & 9 & 13 & 2 & 6 & 37 & 30 & 82 & 13 \\
\hline ALK_B B & 1 & 1 & 5 & 2 & 1 & 4 & 68 & 18 & 80 & 27 \\
\hline PECT̄1_A & 2 & 1 & 19 & 9 & 2 & 11 & 46 & 10 & 87 & 35 \\
\hline PECT1_B & 2 & 1 & 18 & 11 & 2 & 10 & 39 & 17 & 89 & 35 \\
\hline PECT2_A & 2 & 1 & 10 & 13 & 2 & 5 & 39 & 28 & 86 & 35 \\
\hline PECT2_B & 3 & 1 & 13 & 12 & 2 & 6 & 32 & 30 & 86 & 35 \\
\hline CELL_ĀA & 3 & 1 & 5 & 10 & 3 & 6 & 50 & 23 & 83 & 23 \\
\hline $\mathrm{CELL}^{-} \mathrm{B}$ & 3 & 1 & 10 & 10 & 2 & 7 & 37 & 30 & 81 & 27 \\
\hline
\end{tabular}

Rha, rhamnose; Fuc, fucose; Ara, arabinose; Xyl, xylose; Man, mannose; Gal, galactose, Glc, glucose; UA, Uronic acid. _A, RSM was treated after predigesting, _B, RSM was treated before predigesting.

PECT1, PECT2, and CON had less composition shift in these monosaccharides compared to ALK and CELL. Molar percentages of Ara decreased from $25 \%$ to 17\% for CON, from 26 to $18 \%$ for both PECT1_A and PECT1_B, from $16 \%$ to $10 \%$ for PECT2_A, and from $23 \%$ to $13 \%$ for PECT2 B. The changes of Gal were within $2 \%$ for PECT1, $\mathrm{PECT} 2$, and CON. Molar percentage of Glc in PECT1, PECT2, and CON increased by $7 \%, 6 \%$, and $2 \%$ on average, respectively. This does not mean that the microbiota did not ferment these substrates though. Since the recovery of total carbohydrate for these substrates in sacco is $30 \%$ (see above), it means that $70 \%$ has been fermented. Despite this, the remaining carbohydrate has a similar monosaccharide composition as the original substrate that was inserted in the ileum. 


\section{Discussion}

Our in vitro studies on the swine microbiota demonstrated that feeding the microbiota with RSM processed with two kinds of pectinases (PECT1 and PECT2), a cellulase (CELL), or ALK, induced differences in the composition and functionality of the gut microbiota compared to CON. Our findings revealed that ALK and CELL significantly increased the microbial functional abundances related to fibre degradation and also increased SCFA production compared to CON, which did not occur with PECT1 and PECT2. This is in line with the greater reduction of monosaccharide amounts in the nylon bag experiments.

Alpha diversities of ALK and CELL were lower compared to CON, while there were no significant difference among PECT1, PECT2 and CON (Figure 2), which may be due to the selection of particular genera in Actinobacteria and Firmicutes ${ }^{31}$. The abundances of many microbes after feeding with CELL and ALK were significantly higher than for CON, while abundances were not shifted with PECT1 and PECT2 (Figure 4). It has been shown that exogenous carbohydrases from Trichoderma longibrachiatum help in degradation of specific bonds of cell walls either before or after ingestion of the enzyme preparation, and in turn this caused an increase in the numbers and/or activities of bacteria that utilize the polysaccharides in the GIT 13, 32. Supplementation of carbohydrases has been shown to modulate gut microbiota, in various animal models, or in vitro ${ }^{13-15}$. Another possible mode of action of carbohydrases that has been shown in the rumen is that the enzyme preparation alters the fibre structures of substrate and stimulates the attachment of rumen microbiota to feed particles, resulting in improved the fibre degradation ${ }^{14}$. Giraldo et. al (2008) also reported that supplementing carbohydrases directly into the rumen increased the overall fibrolytic activity and stimulated the growth of cellulolytic bacteria ${ }^{15}$. In the current study, CELL treatment (prior to ingestion) significantly increased the numbers of Olsenella, [Eubacterium] nodatum group, Acidaminococcus, Lachnospiraceae NK3A20 group, Bifidobacterium, Acetitomaculum, and Syntrophococcus (Figure 4). These genera may prefer to utilize cellulose- and/or hemicellulose-fragments generated by the action of CELL. However, in the literature only Eubacterium has been reported as cellulolytic ${ }^{33}$. CELL contains multiple glycolytic activities, including exo-1,4- $\beta$-glucanase (cellobiohydrolase), endo-1,4- $\beta$ glucanase, hemicellulase and $\beta$-glucosidase. CELL may have broken down some bonds in cellulose thereby enhancing (hemi)cellulose utilization by the gut bacteria and at the same time also expose other polysaccharides (e.g. pectins) to other bacteria. ALK increased Megasphaera, and Ruminococcus gauvreauii group. Reports show that Ruminococcus are the most commonly cellulolytic organisms ${ }^{33-36}$. Megasphaera is reported to contain glycosyl hydrolase (GH) family 53, which is involved in plant cell wall degradation ${ }^{37}$. These reports indicate that ALK can disrupt the cell wall architecture, by solubilization of polysaccharides by breaking hydrogen bonds and hydrolyzing ester linkages thereby removing esters present as decoration on polysaccharides, making them more accessible for further enzyme degradation and utilization by the microbiota. Interestingly, both PECT1 and PECT2 showed no significant difference in Shannon index compared to CON and a very similar microbiota composition, which seemed to indicate that neither pectinase really changed the cell wall structure, or the changes did not meet the swine gut microbiotas' hydrolytic capacities, and as a result no bacteria were selectively stimulated. We reported before that a cocktail of PECT1 and PECT2 improved degradability of non-glucose polysaccharides of RSM in broilers ${ }^{38-39}$. Nevertheless, it seems that cell wall break down by PECT1 and PECT2 does not lead to advantages for members of the swine gut microbiota. 
The shifts in the bacterial community structures were converted into predicted functional metagenomic profiles (Figure 3,5 and 6C). PECT1 and PECT2 had little effect on the predicted microbial function, as expected, since there were few changes in the microbial structure. In contrast, CELL and ALK had greater microbial composition shifts, subsequently resulting in more changes in microbial function. In the current study, we were interested in fibre utilization, thus, the significantly different microbial functions of carbohydrate metabolism were summarized (Figure 5). The abundances of the carbohydrate related microbial functional metabolism pathways, pyruvate metabolism, propanoate metabolism, pentose phosphate pathway, galactose metabolism, energy metabolism, fructose and mannose metabolism, and carbohydrate digestion and absorption (Figure 5), were higher in CELL and/or ALK compared to CON, while the abundances of lipopolysaccharide biosynthesis and glycan biosynthesis and metabolism were higher in CON compared to ALK. This was corroborated by the SCFA production, which showed that the total SCFA and propionic acid production were significant higher in CELL and ALK compared to CON (Figure 5). Giraldo et al. ${ }^{14}$ reported that supplementation of endoglucanase and xylanase increased propionate production by $28 \%$, and total SCFA production by $11 \%$. Thus, the hypothesis can be entertained that the complementary action between the stimulated microbes and prior incubation with the exogenous enzymes leads to the increase in hydrolytic capacity ${ }^{40}$. However, another study ${ }^{41}$ demonstrated that the concentration of cellulolytic bacteria was not the limiting factor in the digestion of cellulose, and reported that factors associated with the forage and/or the rate of cellulose hydrolysis by cellulase may have a greater influence on the amount of cellulose digested in the rumen. According to the current study, a prerequisite for the complementary action in breaking down recalcitrant fibres might be that the cell wall structure of the substrate is processed appropriately by the feed enzyme, thereby making it more amenable to subsequent degradation by the gut microbial enzymes. HPAEC showed that oligomers of cellulose were detected upon feeding CELL and ALK, which were not seen with PECT1, PECT2 and CON (Figure 8). Our in sacco study also showed that more carbohydrate was utilized upon feeding CELL and ALK, based on dry matter recovery. Moreover, constituent monosaccharide compositions shifted with CELL and ALK. This did not occur with PECT1, PECT2 and CON (Table 2). This can be explained by the fact that CELL and ALK broke down cellulose microfibrils, and stimulated fibrolytic bacteria, which expressed more related enzymes. At the end, more SCFA were produced. This might also be why SCFA production was not increase with PECT1 or PECT2, since no oligomers of pectins were detected in HPAEC (Figure 8). However, the mechanism of exogenous enzymes enhancing degradation of plant cell walls is complex with many interrelated factors, and requires further studies ${ }^{42}$. Moreover, degradation of fibres requires a plethora of microbial enzymes as indicated by the numerous PUL-loci needed by Bacteroides thetaiotaomicron to breakdown pectin ${ }^{42}$. So, this future research needs to elucidate these interrelated factors, since a better understanding of the mode of the action will allow the development of feed enzymes designed specifically to improve feed digestion by swine.

Random Forest analysis found that the microbiota structures were significantly changed after 4 hours fermentation after a shot of processed or non-processed RSM (Figure 6A). This can be explained by comparing this with the results of HPSEC and HPAEC, which showed that almost all the high molecular weight polysaccharides fibres were utilized after 4 hours fermentation (time point $52 \mathrm{~h}$ ), and converted into low molecular weight sugars which are 
substrate for the microbiota. The changes of nutrient composition led to the shifts of microbiota structure. Megasphaera was identified by Random Forest as the most important genus during the classification. Figure 6B shows that a large number of samples contained Megasphaera during the fermentation period. This indicates that Megasphaera might be a microbe, which can utilize the non-processed and processed RSM well. Genome-wide analysis of Megasphaera sp. showed that the genomes had genes coding GH25, GH32, GH43, GH53, GH73 and GH77, which indicate its ability to degrade complex carbohydrates 37,43 . Megasphaera is also known to produce all of the SCFA including valerate ${ }^{37,43-44}$. In the current study, the correlation between SCFA production and relative abundance of genera also showed that Megasphaera had significantly positive correlation with acetic acid and propionic acid (Figure 10). Prevotella 7, and Desulfovibrio were also significantly positively correlated with SCFA production (Figure 10), while Megasphaera and Prevotella 7 had considerably negative correlations with the 'Fate of side chains' (Ara : Gal), and/or had positive correlations with '(Hemi)cellulose versus pectin' $[(\mathrm{Xyl}+\mathrm{Glc}+\mathrm{Man}):(\mathrm{galA}+\mathrm{Gal}$ + Ara + Rha)]. This indicated that these genera had the ability to use the side chain of pectins in the RSM cell wall, and produced SCFA, but only after degradation of the cellulose network, as this was not seen for substrates treated with PECT1 and PECT2. Prevotella 7 and Megasphaera also had significant negative correlation with most of the monosaccharides, which indicated they can use the monosaccharides well. Research showed that Desulfovibrio significantly increased after being fed RG-I-enriched pectin, and SCFA production was also found increased ${ }^{45}$. Prevotella is well known as an important pectinolytic bacterium ${ }^{46-48}$. So, removal of cellulose by CELL seems to increase accessibility of microbial enzymes to pectin. However, in literature, reasoning the other way around is more frequently done: pectinases to remove pectins from the pores from the cell wall enhancing the activity of cellulases ${ }^{30}$.

In conclusion, CELL and ALK feeding considerably changed the swine microbiota structure and predicted functional profile compared to CON, which did not occur with PECT1 and PECT2. The hypothesis is entertained that this results from the different cell wall architecture of RSM once processed by this carbohydrase or alkaline. The increase in relative abundance of pathways involved carbohydrate fermentation in CELL or ALK can be considered as a positive effect of these treatments in fibre utilization and SCFA production. Moreover, it was validated in pigs that CELL and ALK feeding improved the overall degradation of RSM by the mobile nylon bag technique. With all the data collectively considered, we speculate that carbohydrase enzyme, i.e. CELL, improve fibre degradation of RSM during the fermentation by changing the microbial community structure and enzymatic activity and subsequently shifting the microbiota metagenomic functional profile. 


\section{References:}

1. Bouxin, A. Feed \& Food Statistical Yearbook 2018. http://www.fefac.eu. (Accessed 19 December 2019).

2. Karr-Lilienthal, L. K.; Kadzere, C. T.; Grieshop, C. M.; Fahey, G. C., Chemical and nutritional properties of soybean carbohydrates as related to nonruminants: A review. Livest Prod Sci. 2005, 97, 1-12.

3. Simbaya, J.; Slominski, B. A.; Rakow, G.; Campbell, L. D.; Downey, R. K.; Bell, J. M., Quality characteristics of yellow-seeded Brassica seed meals: Protein, carbohydrate, and dietary fiber components. J. Agric. Food Chem. 1995, 43, 2062-2066.

4. Slominski, B. A.; Campbell, L. D., Non-starch polysaccharides of canola meal: Quantification, digestibility in poultry and potential benefit of dietary enzyme supplementation. J. Agric. Food Chem. 1990, 53, 175-184.

5. de Vries, S., Fiber fermentation in pigs and poultry: sense and nonsense of its manipulation. Doctoral dissertation, Wageningen University \& Research, Wageningen, 2014.

6. $\quad$ Pustjens, A. M.; Schols, H. A.; Kabel, M. A.; Gruppen, H., Characterisation of cell wall polysaccharides from rapeseed (Brassica napus) meal. Carbohydr Polym. 2013, 98, 1650-6

7. $\quad$ Siddiqui, I. R.; Wood, P. J., Carbohydrates of rapeseed: a review. J. Sci. 1977, 28, 530-538.

8. Slominski, B. A., Hydrolysis of galactooligosaccharides by commercial preparations of $\alpha$-galactosidase and $\beta$-fruetofuranosidase: Potential for use as dietary additives. J. Sci. 1994, 65, 323-330.

9. Meng, X.; Slominski, B., Nutritive values of corn, soybean meal, canola meal, and peas for broiler chickens as affected by a multicarbohydrase preparation of cell wall degrading enzymes. Poult. Sci. 2005, 84, 12421251.

10. Pustjens, A. M.; de Vries, S.; Bakuwel, M.; Gruppen, H.; Gerrits, W. J.; Kabel, M. A., Unfermented recalcitrant polysaccharide structures from rapeseed (Brassica napus) meal in pigs. Ind Crops Prod. 2014, 58, 271279.

11. Staack, L.; Della Pia, E. A.; Jørgensen, B.; Pettersson, D.; Pedersen, N. R., Cassava cell wall characterization and degradation by a multicomponent NSP-targeting enzyme (NSPase). Sci. Rep. 2019, 9, 1-11.

12. Flint, H. J.; Scott, K. P.; Duncan, S. H.; Louis, P.; Forano, E., Microbial degradation of complex carbohydrates in the gut. Gut Microbes. 2012, 3, 289-306.

13. Apajalahti, J.; Kettunen, A.; Graham, H., Characteristics of the gastrointestinal microbial communities, with special reference to the chicken. Worlds Poult. Sci. J. 2004, 60, 223-232.

14. Högberg, A.; Lindberg, J. E., Influence of cereal non-starch polysaccharides and enzyme supplementation on digestion site and gut environment in weaned piglets. Anim. Feed Sci. Technol. 2004, 116, 113128.

15. Kiarie, E.; Nyachoti, C. M.; Slominski, B. A.; Blank, G., Growth performance, gastrointestinal microbial activity, and nutrient digestibility in early-weaned pigs fed diets containing flaxseed and carbohydrase enzyme1,2. Anim. Sci. J. 2007, 85, 2982-2993.

16. Nsereko, V.; Beauchemin, K.; Morgavi, D.; Rode, L.; Furtado, A.; McAllister, T.; Iwaasa, A.; Yang, W.; Wang, Y., Effect of a fibrolytic enzyme preparation from Trichoderma longibrachiatum on the rumen microbial population of dairy cows. Can. J. Microbiol. 2002, 48, 14-20.

17. Giraldo, L.; Ranilla, M.; Tejido, M.; Carro, M., Influence of exogenous fibrolytic enzymes and fumarate on methane production, microbial growth and fermentation in Rusitec fermenters. Br. J. Nutr. 2007, 98, 753-761.

18. Giraldo, L.; Tejido, M.; Ranilla, M.; Ramos, S.; Carro, M., Influence of direct-fed fibrolytic enzymes on diet digestibility and ruminal activity in sheep fed a grass hay-based diet. Anim. Sci. J. 2008, 86, 1617-1623.

19. Long, C.; de Vries, S.; Venema, K., Polysaccharide source altered ecological network, functional profile, and short-chain fatty acid production in a porcine gut microbiota. Benef. Microbes. https://doi.org/10.3920/BM2020.0006. 2020.

20. Sáyago-Ayerdi, S. G.; Zamora-Gasga, V. M.; Venema, K., Prebiotic effect of predigested mango peel on gut microbiota assessed in a dynamic in vitro model of the human colon (TIM-2). Int. Food Res. J. 2017, 118, 8995.

21. Gibson, G. R.; Cummings, J. H.; Macfarlane, G. T., Use of a three-stage continuous culture system to study the effect of mucin on dissimilatory sulfate reduction and methanogenesis by mixed populations of human gut bacteria. Appl. Environ. Microbiol. 1988, 54, 2750-2755.

22. $\quad$ Maathuis, A.; Hoffman, A.; Evans, A.; Sanders, L.; Venema, K., The effect of the undigested fraction of maize products on the activity and composition of the microbiota determined in a dynamic in vitro model of the human proximal large intestine. J Am Coll Nutr. 2009, 28, 657-666.

23. Li, S.; Sauer, W.; Hardin, R., Effect of dietary fibre level on amino acid digestibility in young pigs. Can. J. Anim. Sci. 1994, 74, 327-333.

24. Thacker, P.; Qiao, S., Further modifications to the mobile nylon bag technique to determine nutrient 
digestibility for swine. Asian-Australas J Anim Sci. 2001, 14, 1149-1156.

25. Klindworth, A.; Pruesse, E.; Schweer, T.; Peplies, J.; Quast, C.; Horn, M.; Glöckner, F. O., Evaluation of general 16S ribosomal RNA gene PCR primers for classical and next-generation sequencing-based diversity studies. Nucleic Acids Res. 2013, 41 (1), e1-e1.

26. Bolyen, E.; Rideout, J. R.; Dillon, M. R.; Bokulich, N. A.; Abnet, C. C.; Al-Ghalith, G. A.; Alexander, H.; Alm, E. J.; Arumugam, M.; Asnicar, F., Reproducible, interactive, scalable and extensible microbiome data science using QIIME 2. Nat. Biotechnol. 2019, 37, 852-857.

27. Callahan, B. J.; McMurdie, P. J.; Rosen, M. J.; Han, A. W.; Johnson, A. J. A.; Holmes, S. P., DADA2: High-resolution sample inference from Illumina amplicon data. Nature Methods. 2016, 13, 581.

28. Douglas, G. M.; Maffei, V. J.; Zaneveld, J.; Yurgel, S. N.; Brown, J. R.; Taylor, C. M.; Huttenhower, C.; Langille, M. G., PICRUSt2 for prediction of metagenome functions. Nat. Biotechnol. 2020, 1-5.

29. Englyst, H. N.; Cummings, J. H., Simplified method for the measurement of total non-starch polysaccharides by gas-liquid chromatography of constituent sugars as alditol acetates. Analyst. 1984, 109, 937-942.

30. Ahmed, A. E. R.; Labavitch, J. M., A simplified method for accurate determination of cell wall uronide content. J. Food Biochem. 1978, 1, 361-365.

31. Rösch, C.; Taverne, N.; Venema, K.; Gruppen, H.; Wells, J. M.; Schols, H. A., Effects of in vitro fermentation of barley $\beta$-glucan and sugar beet pectin using human fecal inocula on cytokine expression by dendritic cells. Mol. Nutr. Food Res. 2017, 61, 1600243.

32. Parks, D. H.; Tyson, G. W.; Hugenholtz, P.; Beiko, R. G., STAMP: statistical analysis of taxonomic and functional profiles. Bioinformatics 2014, 30, 3123-3124.

33. Ramasamy, U. S.; Venema, K.; Schols, H. A.; Gruppen, H., Effect of soluble and insoluble fibers within the in vitro fermentation of chicory root pulp by human gut bacteria. J. Agric. Food Chem. 2014, 62, 6794-802.

34. Umu, O. C.; Frank, J. A.; Fangel, J. U.; Oostindjer, M.; da Silva, C. S.; Bolhuis, E. J.; Bosch, G.; Willats, W. G.; Pope, P. B.; Diep, D. B., Resistant starch diet induces change in the swine microbiome and a predominance of beneficial bacterial populations. Microbiome. 2015, 3, 16.

35. Beauchemin, K.; Colombatto, D.; Morgavi, D.; Yang, W.; Rode, L., Mode of action of exogenous cell wall degrading enzymes for ruminants. Can. J. Anim. Sci. 2004, 84, 13-22.

36. Robert, C.; Bernalier-Donadille, A., The cellulolytic microflora of the human colon: evidence of microcrystalline cellulose-degrading bacteria in methane-excreting subjects. FEMS Microbiol. Ecol. 2003, 46, 8189.

37. Wedekind, K. J.; Mansfield, H. R.; Montgomery, L., Enumeration and isolation of cellulolytic and hemicellulolytic bacteria from human feces. Appl. Environ. Microbiol. 1988, 54, 1530-1535.

38. Kopečný, J.; Hajer, J.; Mrázek, J., Detection of cellulolytic bacteria from the human colon. Folia Microbiol. 2004, 49, 175.

39. Flint, H. J.; Bayer, E. A.; Rincon, M. T.; Lamed, R.; White, B. A., Polysaccharide utilization by gut bacteria: potential for new insights from genomic analysis. Nat. Rev. Microbiol. 2008, 6, 121-131.

40. $\quad$ Shetty, S. A.; Marathe, N. P.; Lanjekar, V.; Ranade, D.; Shouche, Y. S., Comparative genome analysis of Megasphaera sp. reveals niche specialization and its potential role in the human gut. PloS one 2013, 8, e79353.

41. De Vries, S.; Pustjens, A.; Kabel, M.; Kwakkel, R.; Gerrits, W., Effects of processing technologies and pectolytic enzymes on degradability of nonstarch polysaccharides from rapeseed meal in broilers. Poult. Sci. 2014, 93, 589-598

42. Pustjens, A. M.; de Vries, S.; Gerrits, W. J.; Kabel, M. A.; Schols, H. A.; Gruppen, H., Residual carbohydrates from in vitro digested processed rapeseed (Brassica napus ) meal. J. Agric. Food Chem. 2012, 60, 8257-63.

43. de Vries, S.; Pustjens, A. M.; Kabel, M. A.; Salazar-Villanea, S.; Hendriks, W. H.; Gerrits, W. J., Processing technologies and cell wall degrading enzymes to improve nutritional value of dried distillers grain with solubles for animal feed: an in vitro digestion study. J. Agric. Food Chem. 2013, 61, 8821-8828.

44. Morgavi, D.; Beauchemin, K.; Nsereko, V.; Rode, L.; Iwaasa, A.; Yang, W.; McAllister, T.; Wang, Y., Synergy between ruminal fibrolytic enzymes and enzymes from Trichoderma longibrachiatum. J. Dairy Sci. 2000, $83,1310-1321$

45. Dehority, B.; Tirabasso, P., Effect of ruminal cellulolytic bacterial concentrations on in situ digestion of forage cellulose. Anim. Sci. J. 1998, 76, 2905-2911.

46. Luis, A. S.; Briggs, J.; Zhang, X.; Farnell, B.; Ndeh, D.; Labourel, A.; Baslé, A.; Cartmell, A.; Terrapon, N.; Stott, K., Dietary pectic glycans are degraded by coordinated enzyme pathways in human colonic Bacteroides. Nat. Microbiol. 2018, 3, 210-219.

47. Marounek, M.; Fliegrova, K.; Bartos, S., Metabolism and some characteristics of ruminal strains of Megasphaera elsdenii. Appl. Environ. Microbiol. 1989, 55, 1570-1573.

48. Chiquette, J. In The role of probiotics in promoting dairy production, 30 th Western Nutrition Conference, 2009; $\mathrm{p} 2$. 
49. Mao, G.; Li, S.; Shen, X.; Zhou, S.; Orfila, C.; Linhardt, R. J.; Ye, X.; Chen, S., Depolymerized RG-I enriched pectin from citrus segment membrane modulates gut microbiota, increases SCFAs production, promotes the growth of Bifidobacterium spp., Lactobacillus spp. and Faecalibaculum spp. Food Funct. 2019, 10, 7828-7843. 50. Marounek, M.; Kalachnyuk, G., Stoichiometry of pectin and glucose fermentation in Prevotella ruminicola. Ukrainskii biokhimicheskii zhurnal (1978). 1995, 67, 107-110.

51. Nograšek, B.; Accetto, T.; Fanedl, L.; Avguštin, G., Description of a novel pectin-degrading bacterial species Prevotella pectinovora sp. nov., based on its phenotypic and genomic traits. J. Microbiol. 2015, 53, 503-510. 52. Tian, L.; Bruggeman, G.; van den Berg, M.; Borewicz, K.; Scheurink, A. J.; Bruininx, E.; de Vos, P.; Smidt, H.; Schols, H. A.; Gruppen, H., Effects of pectin on fermentation characteristics, carbohydrate utilization, and microbial community composition in the gastrointestinal tract of weaning pigs. Mol. Nutr. Food Res. 2017, 61, 1600186. 


\section{Supplemental figures:}

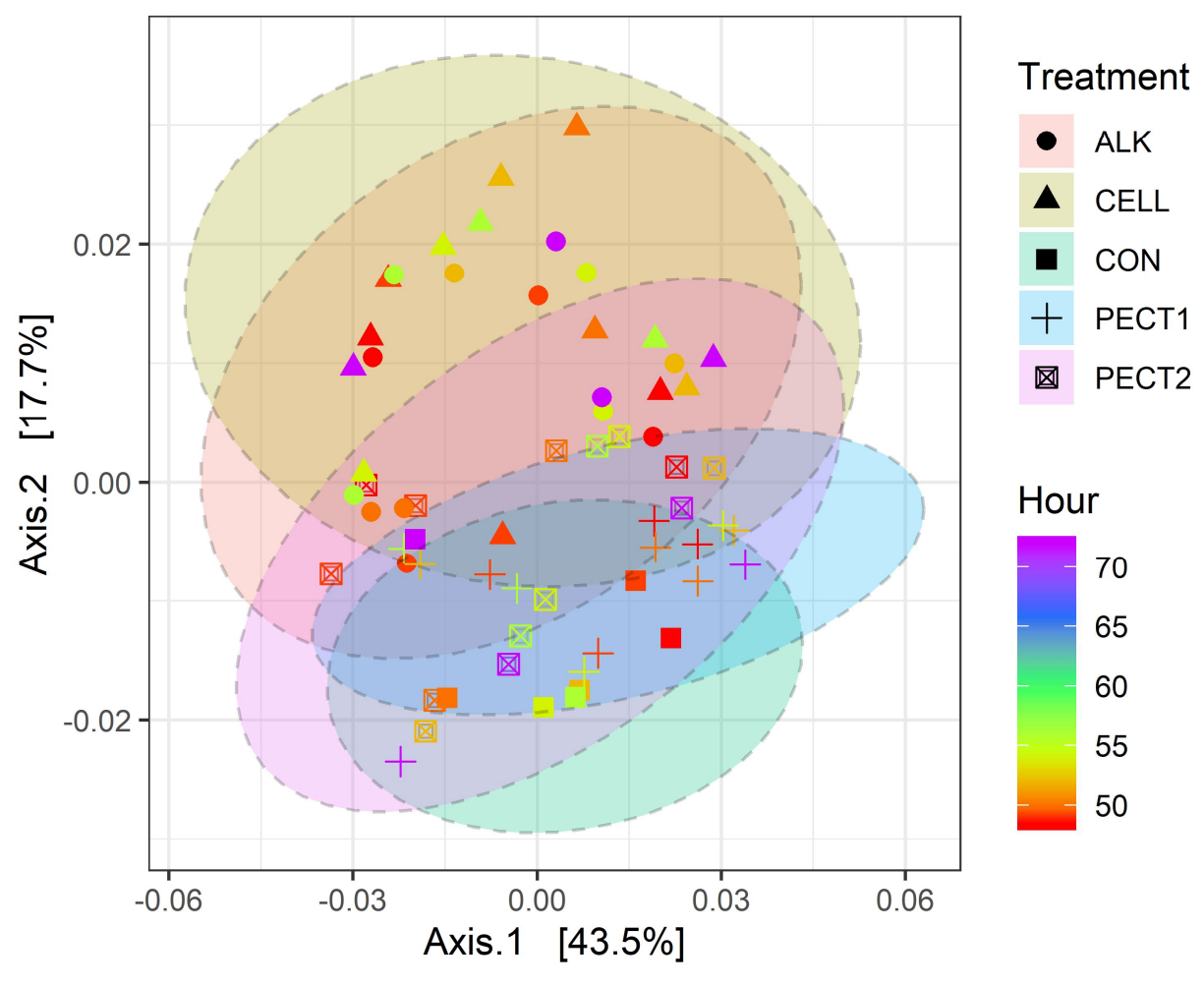

Figure S1. Principle coordinate analysis (PCoA) plot generated based on the calculated distances in the weighted matrix. Samples were grouped by shape and colour in terms of treatment and time point, respectively: non-processed RSM (CON), square; RSM processed by $6 \mathrm{M} \mathrm{NaOH}$ (ALK), sphere; RSM processed by Pectinex Ultra SP (PECT1), plus; RSM processed by Multifect Pectinase (PECT2), square with cross inside; RSM processed by Accellerase 1000 (CELL), triangle; a red-green-purple scale was used to indicate the fermentation time (red and purple depict start and end of the fermentation period). 


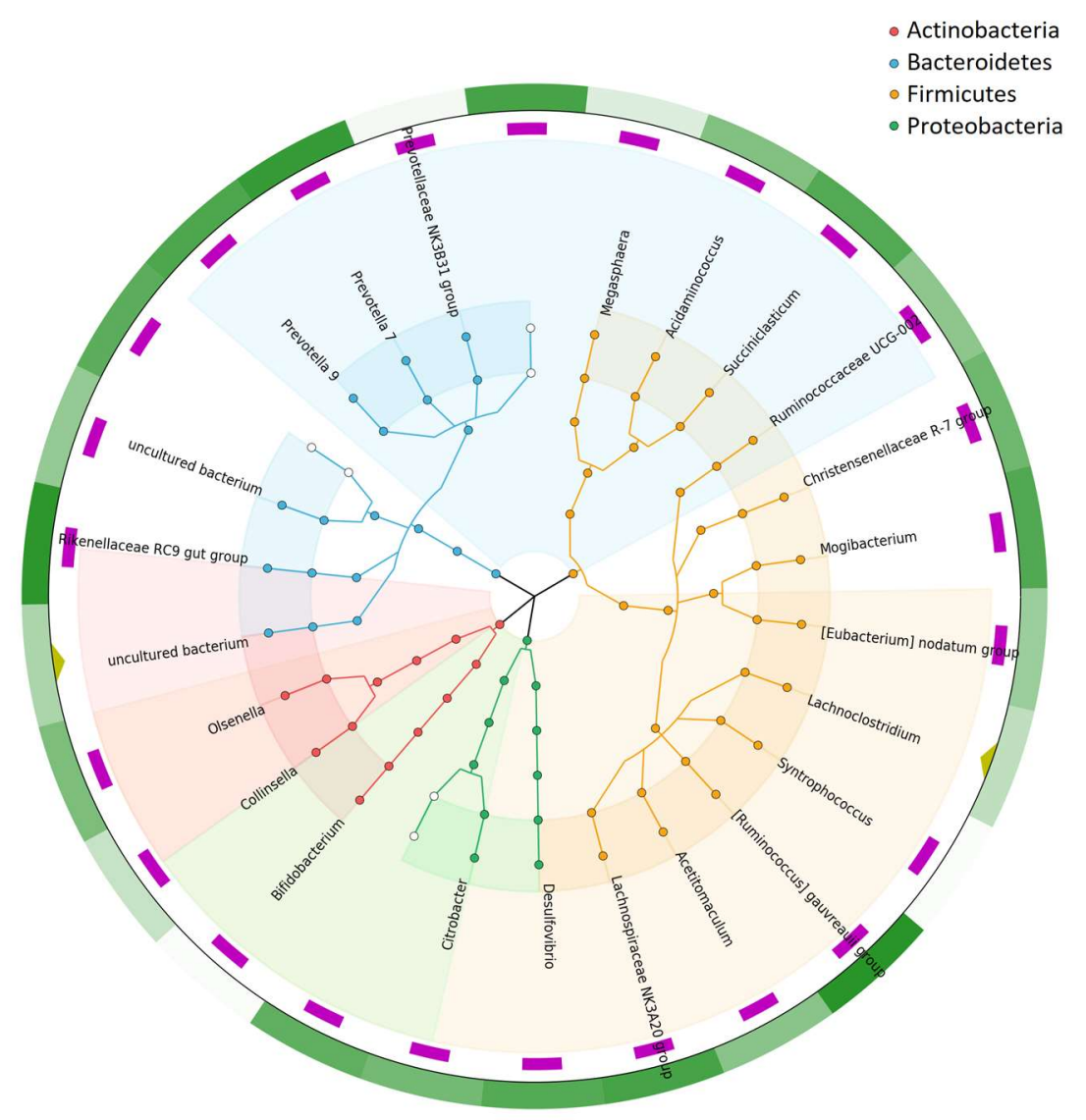

Figure S2. Phylogenetic tree of the core bacteria fed with non-modified and modified RSM. The table underlying the tree was normalized via division by the sum of sequences in a given sample and multiplied by the minimum sum across all samples. Relative abundances were filtered as following: values below a relative abundance threshold of $0.01 \%$ were not taken into account; taxa with a median relative abundance $<1 \%$ in all groups were not considered for statistical analysis; purple squares represents the relative abundance of the ASVs higher than $0.5 \%$, while the yellow triangle stands for those lower than $0.5 \%$. The outer ring heatmap stands for the microbial relative abundance. Light green indicates lower abundance, while dark green represents higher abundance. 

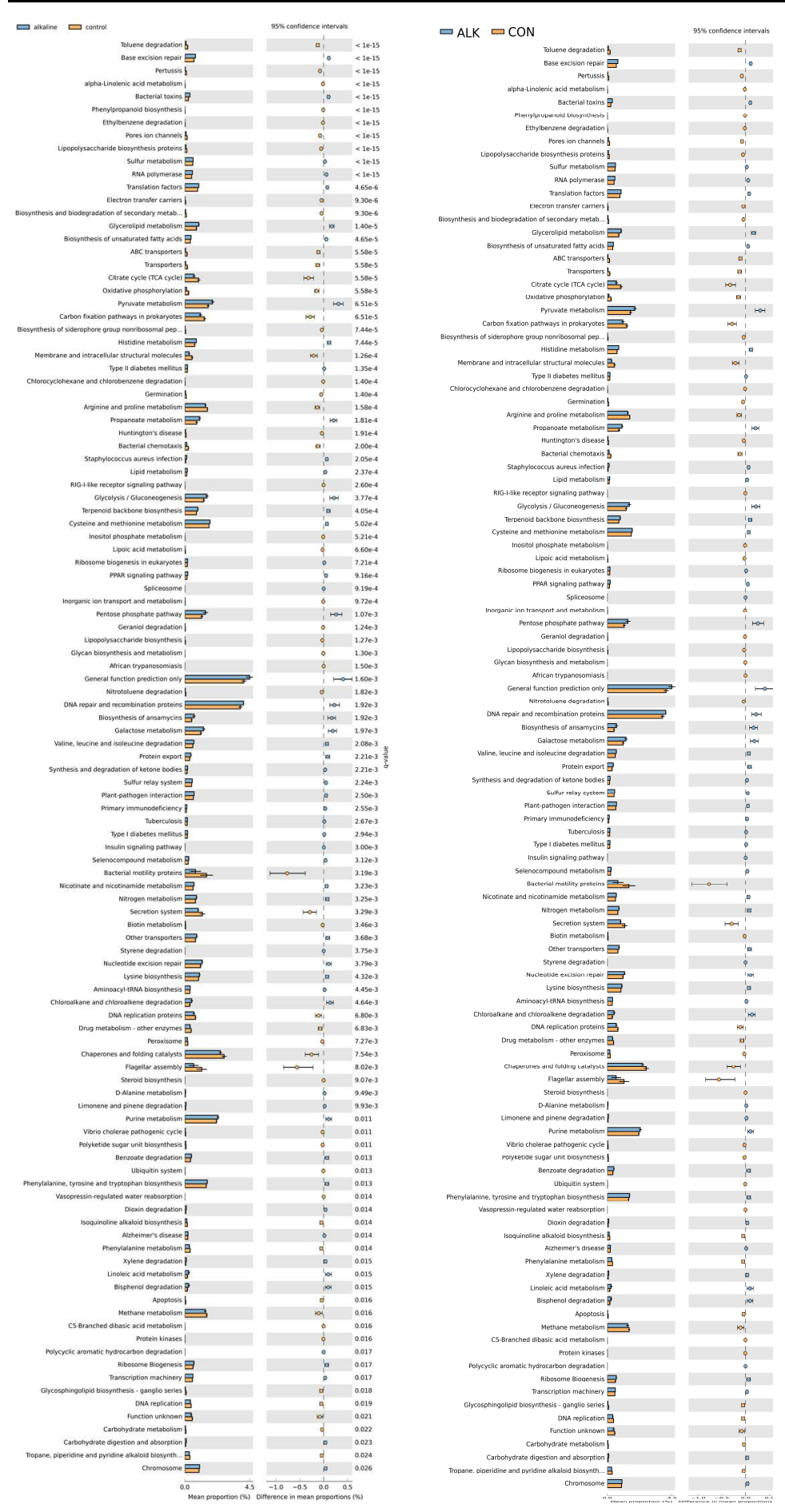
Figure S3: Significantly different metagenomic function in relative abundance in different treatment compared to $\mathrm{CON}$

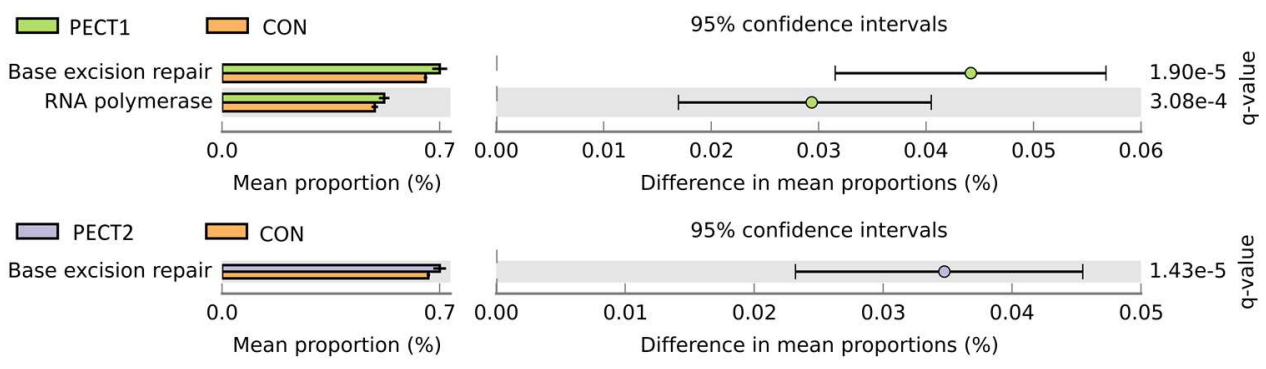

Figure S3: Significantly different metagenomic function in relative abundance in different treatment compared to $\mathrm{CON}$ 
Chapter 5 


\section{Chapter 6}

\section{High-throughput screening of structures of plant cell wall polysaccharides using a novel method}

Cheng Long ${ }^{1,2}$, Koen Venema ${ }^{1,2^{*}}$

${ }^{1}$ Faculty of Science and Engineering, Centre for Healthy Eating \& Food Innovation, Maastricht University - campus Venlo, The Netherlands

${ }^{2}$ School of Nutrition and Translational Research in Metabolism (NUTRIM), Maastricht University, The Netherlands

\section{"Correspondence:}

Maastricht University - campus Venlo, Centre for Healthy Eating \& Food Innovation, St. Jansweg 20, 5928 RC Venlo, The Netherlands

k.venema@maastrichtuniversity.nl; tel.: +31622435111

Prepared for publication 


\begin{abstract}
The aim of the study was to develop a methodology, which enables to detect and determine plant cell wall structures in plant materials. The technique (glycome profiling of plant cell wall polysaccharides, or Glycoprofiling) is developed with the use of carbohydrate binding modules (CBMs) with unique specificities for plant cell wall polysaccharides, linked to a green fluorescent protein (GFP) as reporter. Different plasmid constructs with 35 different CBMs were made. These were overexpressed in E.coli and purified with the use of a HIStag. The fusion proteins were then tested against a panel of 40 carbohydrates. When used on plant material, the profiling results would provide a global overview of the cell wall polysaccharide composition and structure. The toolbox can be used to extend the monoclonal antibody repertoir that we used in Chapter 3, and can easily be extended with additional CBM-fusion proteins. Moreover, fusion proteins with different colors (e.g. m-Cherry (red), YFP (yellow) or BFP (blue)) can be made by simple exchange with GFP. This toolbox may become essential in the study of the dynamics of breakdown of dietary fibres by the (pig) gut microbiota.
\end{abstract}




\section{Introduction}

Plant cell wall polysaccharides are composed of (hemi)celluloses and pectins. Cellulose is crystalline and strong, thus it is an important structural component of the primary cell wall in green plants. Hemicelluloses bind with pectin and cellulose to form a network of cross-linked fibres. Pectins are linked to each other and cross-linked between pectin and hemicellulose, and between pectin and cellulose (1). Pectins consist of homogalacturonan (HG), rhamnogalacturonan I and II (RG-I and RG-II), xylogalacturonan, arabinogalactan type I and type II (AG-I and AG-II) and arabinan. Hemicelluloses are composed of xylan, xyloglucan, mannan, and $\beta$-glucan. The plant cell wall is a potential energy source as animal feed for livestock. However, plant cell wall polysaccharides cannot be utilized by endogenous enzymes in animal, and also can only be partly degraded by gut microbiota. Therefore, in order to develop new methods to improve the degradability of plant cell wall polysaccharides, a better understand of the cell wall polysaccharide composition and structure and their interactions are essential.

One of the promising high-throughput way to screen the polysaccharide structure in plant cell wall is by the use of carbohydrate binding modules (CBMs) and/or monoclonal antibodies (mAbs) (2). CBMs are the non-catalytic part of cell-wall-degrading enzymes, and they are attached to the glycoside hydrolase $(\mathrm{GH})$ catalytic modules. Usually, CBMs have to recognize and bind to the specific polysaccharides first, before the GHs cleave the polysaccharides (3). CBMs are grouped into amino acid sequence-based families in the Carbohydrate-Active EnZymes (CAZy) database (http://www.cazy.org/) (4), and currently, there are $87 \mathrm{CBM}$ families in the database. CBMs have been applied to recognize xylans (5), cellulose $(6,7)$, and mannans (7) directly in plant materials.

Another important tool for study of plant cell wall structure is using immunohistochemical techniques (e.g. mAbs) (8), which is developed from neoglycoprotein immunization procedures or from retrospective characterization after immunization with complex cell wallderived materials (9). Repertoires of cell wall-directed monoclonal antibodies are being assembled. For example, the CarboSource Services, of the Complex Carbohydrate Research Center, University of Georgia, USA (https://www.ccrc.uga.edu/ carbosource/CSS_home.html), PlantProbes, of the University of Leeds. UK (http://www.plantprobes.net), Biosupplies Australia (http://www.biosupplies.com.au), or the WallBioNet: Plant Cell Wall Biosynthesis Research Network (http://glycomics.ccrc.uga.edu/wall2/index.html). mAbs have been utilized to bind epitopes present on RG-I (10), homogalacturonan (11), xylogalacturonan (12), xylans (13), xyloglucan (14), arabinoxylans (13), and arabinogalactan (15). We used a collection of these mAbs in Chapter 3 to study the dynamic fermentation of rapeseed meal fibres by the pig microbiota.

The aim of the study was to create a set of fusion proteins with 35 different CBMs and green fluorescent protein (GFP) as a reporter. This (initial) toolbox with CBM-GFP fusions extends the monoclonal antibodies that we have used in Chapter 3 of this thesis. 
Materials and methods

Carbohydrate binding modules, CBMs

Cloning The nucleotide sequence of CBM domains were downloaded from NCBI and the genes were synthetized by BaseClear B.V. (Leiden, The Netherlands). Care was taken that no BmHI, EcoRI or KpnI sites were present in the synthesized genes, as these restriction sites were used for cloning. When these sites were present, the nucleotide sequenced was changed such that this did not lead to an amino acid change in the expressed fusion protein. The synthetic genes contained a BamHI or EcoRI site on the 5'-end and a KpnI site on the 3-end. A list of CBM domains and their (predicted) specificities is shown in Supplementary Table 1. The gene fragments were cloned using standard procedures into pET 28c (containing the green fluorescent protein gene (GFP) with an N-terminal six-histidine tag) using the 5' BamHI or EcoRI and 3' KpnI sites and transformed into E.coli DH5 $\alpha$. The reconstructed expression vectors encode GFP + CBMs fusion proteins (Figure 1). The DNA sequence fidelity of all constructs was verified by bidirectional sequencing by BaseClear.

Plasmids DNA electrophoresis plasmid DNA was isolated from E.coli transformants using QIAprep Spin Miniprep Kit (QIAGEN, Venlo, The Netherlands). Restriction enzymes (BanHI and KpnI) were used to cut out the insert fragment in $2 \mathrm{~h}$ at $37^{\circ} \mathrm{C}$ in $50 \mu \mathrm{L}$ of reaction buffer. Then, the fragments size was assessed using the Bioanalyzer Instrument (Agilent, California, United States).

Protein overexpression and purification All verified constructs were transformed into the expression strain, Escherichia coli BL 21 (DE3). Next, $250 \mathrm{~mL}$ of Luria-Bertani broth, containing $50 \mu \mathrm{g} / \mathrm{ml}$ kanamycin, was inoculated with the transformed cells and incubated at $37^{\circ} \mathrm{C}$. Once an optical density of 0.6 at $600 \mathrm{~nm}$ was reached, expression of the recombination protein was induced by adding isopropyl 1-thio- $\beta$-D-galactopyranoside (IPTG) to a final concentration of $1 \mathrm{mM}$. The cultures were incubated for an additional $5 \mathrm{~h}$ at $17{ }^{\circ} \mathrm{C}$. Cells were harvest by centrifugation at $4000 \mathrm{x}$ g for $20 \mathrm{~min}$. The His-tagged fusion proteins were subsequently purified by using the QIAexpress ${ }^{\circledR}$ Ni-NTA Fast Start kit (Qiagen, Venlo, The Netherlands) according to the manufacturer's protocol.

SDS-PAGE and Coomassie Staining The molecular weight of the purified overexpressed proteins was determined the proteins molecular weight using SDS-PAGE according to the manufacturer's protocol (Bio-Rad Laboratories, California, United States). SDS-PAGE gel was stained with Coomassie staining solution (Bio-Rad Laboratories).

Glycome profiling (Glycoprofiling)of polysaccharide panel

Polysaccharides Polysaccharides from various plant sources were obtained from Megazyme (Bray, Wicklow, Ireland) and Sigma (St. Louis, Missouri, Unites States) (Table S1). Stock solutions were prepared by solubilizing the polysaccharides at $1 \mathrm{mg} / \mathrm{mL}$ in deionized water and storing these stock solutions at $-20^{\circ} \mathrm{C}$.

Glycoprofiling Samples prepared above were applied $(50 \mu \mathrm{L}$ of $100 \mu \mathrm{g} / \mathrm{mL}$ in deionized water per well) in duplicate to Corning ${ }^{\circledR} 96$ well black plates (Corning Inc., New York, United States) and dried to the well surfaces by evaporation overnight at $37^{\circ} \mathrm{C}$. Control wells contained deionized water. The plates were then blocked with $200 \mu \mathrm{L}$ of $11 \%(\mathrm{w} / \mathrm{v})$ bovine serum albumin (BSA) in Tris-buffered saline $(50 \mathrm{mM}$ Tris-HCl, pH 7.6, containing $100 \mathrm{mM}$ sodium chloride) for $1 \mathrm{~h}$. The blocking agent was removed by aspiration, and $50 \mu \mathrm{l}$ of purified CBM-GFP fusion proteins were added to each well and incubated for $1 \mathrm{~h}$ at room temperature. Subsequently, the supernatant was removed and wells were washed three times with $200 \mu \mathrm{L}$ of $0.1 \%(\mathrm{w} / \mathrm{v})$ BSA in Tris-buffered saline (wash buffer). Next, CBM proteins (50 $\mu \mathrm{l})$ were added to a well as positive control (maximum fluorescence). The GFP fluorescence of each 
microplate well was subsequently read by using a multi-mode microtiter plate reader (BioTek Synergy HTX, BioSPX, Abcoude, The Netherlands).

\section{Result and discussion}

The synthesized CBM genes were inserted into pET28c vector, containing the GFP gene with an N-terminal six-histidine tag (see Figure 1 for the case of CBM27). In total, 35 CBM genes were used to reconstruct fusions with GFP in the expression vector. These were CBM2, CBM3, CBM6, CBM4-9-1, CBM4-9-2, CBM11, CBM13, CBM13-1, CBM13-2, CBM13-3, CBM13-4, CBM15, CBM17, CBM20, CBM21, CBM26,CBM27, CBM29-65-1, CBM2965-2, CBM35, CBM36, CBM39, CBM41, CBM47, CBM51, CBM58, CBM60, CBM61, CBM62, CBM63, CBM66, CBM67, CBM71, CBM80 and CBM81(Supplemetary Table 1). The sizes of the inserted fragments in the plasmids were assessed by electrophoresis, which showed that the insert size of each CBM was consistent with their gene size (for an example see Figure 2). Constructs with the correct size were subsequently verified by bidirectional sequencing. The results of SDS-PAGE of the Ni-column purified proteins (Figure 3) also revealed that the protein molecular weight of each CBM-GFP fusion was in line with their predicted size (using their gene maps).

The key steps of the Glycoprofiling technique are summarized in Figure 4 and can be divided into three stages: polysaccharide immobilization, epitope recognition by CBM and binding of the CB-GFP fusion protein, and fluorescence reading. The type of 96-well plate was selected based on literature (16), where the polysaccharides can optimally immobilize on the surface of the bottom of the plate.

The reproducibility of the Glycoprofiling technique was tested by comparing data derived from duplicated wells. The results shown in Table 1 indicate that CBM27, which has the specificity to recognize $\alpha$-D-(1-6)-Galp and $\beta$-D-(1-4)-Manp, binds to xylan(beechwood), galactomannan(carob), galactomannan, guar galactomannan, D-galacto-D-mannan, and locust beangum. The coefficient of variation $(\mathrm{CV})$ of fluorescence values for these polysaccharides were $7.9 \%, 10.9 \%, 5.2 \%, 8.3 \%, 3.4 \%$, and $5.7 \%$, respectively. The low CV values indicated high reproducibility. Furthermore, the rest of the 35 polysaccharides showed no binding, which indicated the high specificity of the CBMs (Table S2). The maximum fluorescence signal for CBM27 was on average 20.5 (Table S1, positive control), indicating particularly strong binding of CBM27 to D-galacto-D-mannan (same average fluorescence signal as for positive control; Table 1). Although it is not possible to conclude this for all fusion proteins, the fact that the CBM27-GFP fusion still recognizes its (predicted) target, shows that at least this fusion protein correctly folds into its separate domains (GFP and CBM). Care was taken in constructing the fusion proteins to include a few amino acids more than just the CBM domain, which could function as a sort of linker between the two domains in the fusion. Future analysis will have to show whether the other fusion proteins are also correctly folded. In any case, all fusion proteins fluoresced green upon excitation (data not shown) suggesting correct folding of the other fusion proteins as well.

When used on plant material (such as rapeseed meal), the profiling results would provide a global overview of the cell wall polysaccharide composition and structure. The toolbox can be used to extend the monoclonal antibody repertoir that we used in Chapter 3, and can easily be extended with additional CBM-fusion proteins. Moreover, fusion proteins with different colors (e.g. m-Cherry (red), YFP (yellow) or BFP (blue)) can be made by simple exchange with GFP. This toolbox may become essential in the study of the dynamics of breakdown of dietary fibres by the (pig) gut microbiota. 


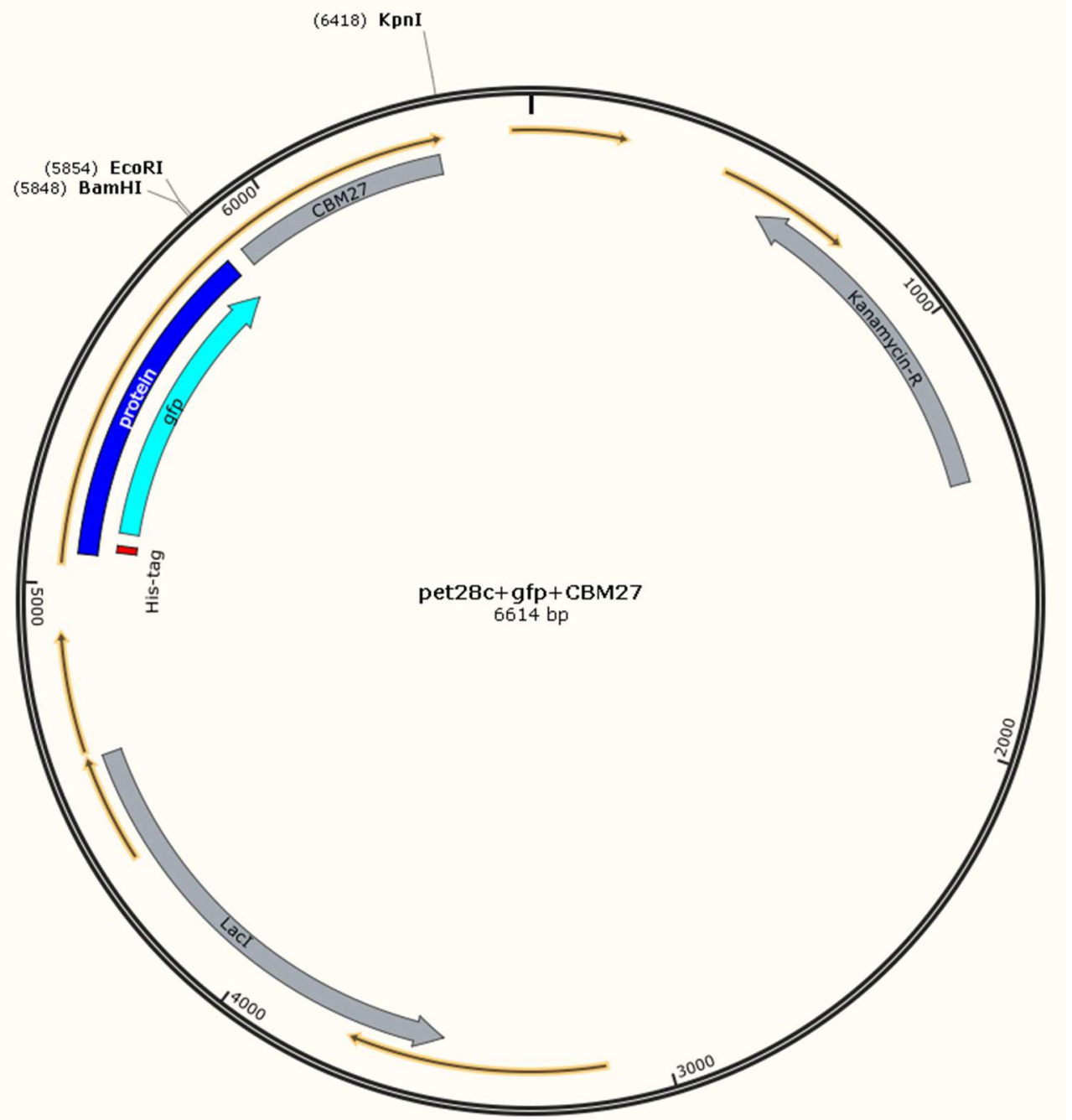

Figure 1. An example of plasmid gene map (CBM27). 


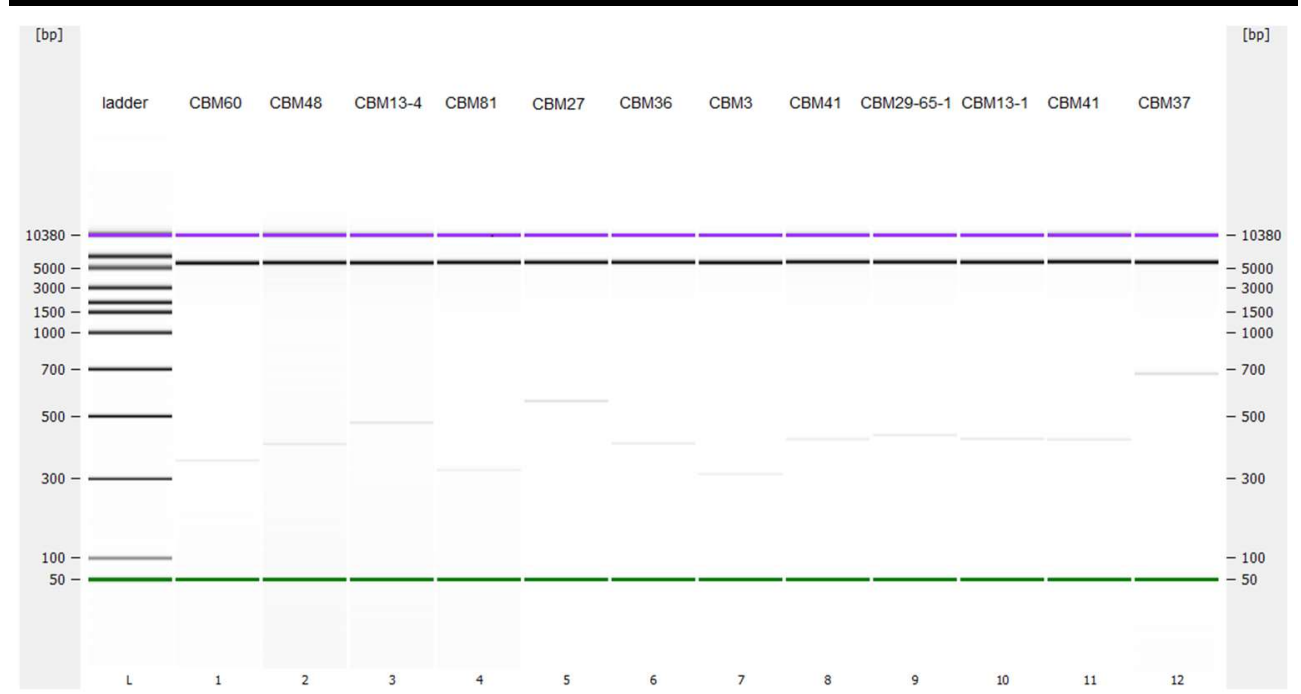

Figure 2. CBM-Plasmid DNA electrophoretic image in the Bioanalyzer for 12 representative CBM constructs after digesting with BamHI and KpnI. The black colored-bands are the vector, the light-grey colored bands of different size in each lane are the respective CBM genes. The blue and green colored bands are the internal standaards for the Bioanalyzer.

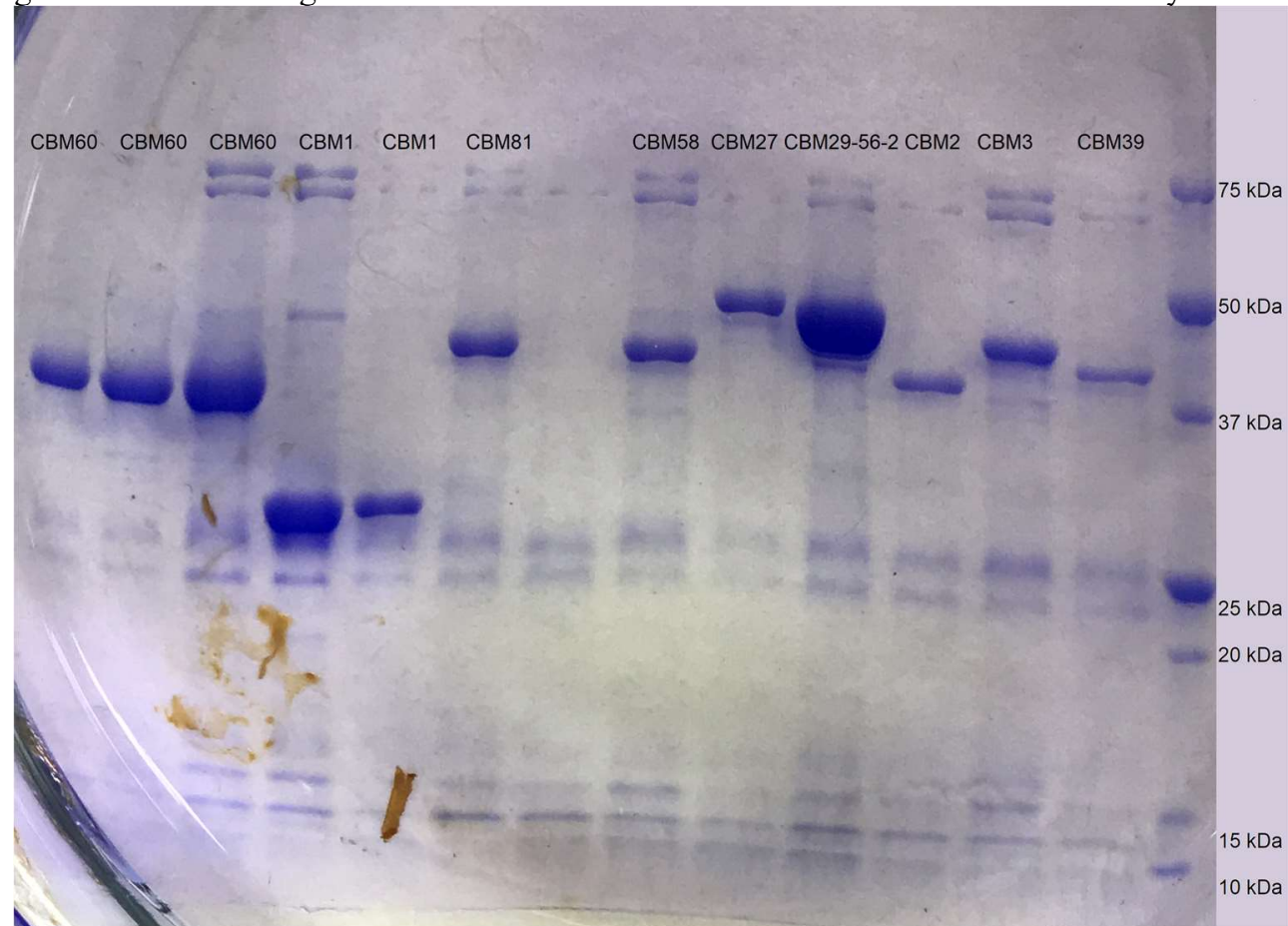

Figure 3. SDS-PAGE analysis on the expression of representiative recombinant CBM-GFP fusionis. Multiple leanes with the same CBM-code indicate separate eluted fractions collected from the Ni-column. 
(६

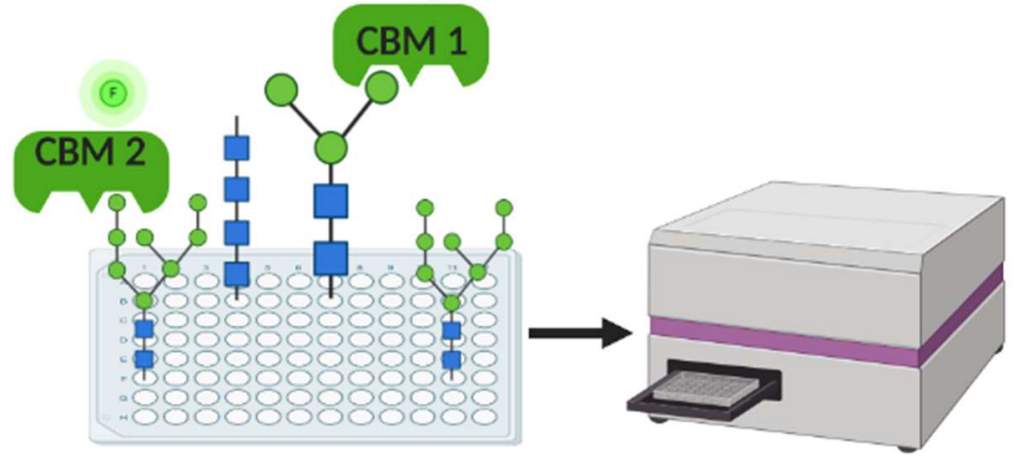

Figure 4. Schematic representation of fluorescence reading in Glycoprofiling.

Table 1. Fluorescence values due to binding of CBM27 to polysaccharides when tested in the Glycoprofiling.

\begin{tabular}{|l|l|l|l|}
\hline & CBM27-1 & CBM27-2 & C.V. \% \\
\hline Guar medium visicosity xylan(beechwood) & 17 & 19 & 7.9 \\
\hline Galactomannan(carob) high viscosity & 14 & 12 & 10.9 \\
\hline Guar galactomannan(GD21) enzyme modified guar & 14 & 13 & 5.2 \\
\hline Guar galactomannan (GD 28) enzyme modified guar & 18 & 16 & 8.3 \\
\hline D-galacto-D-mannan from ceratonia & 21 & 20 & 3.4 \\
\hline Locust beangum from ceratonia siliqua seeds & 13 & 12 & 5.7 \\
\hline
\end{tabular}




\section{References:}

1. Broxterman SE, Schols HA. Interactions between pectin and cellulose in primary plant cell walls. Carbohydr. Polym. 2018;192:263-72.

2. Moller I, Sorensen I, Bernal AJ, Blaukopf C, Lee K, Obro J, et al. High-throughput mapping of cell-wall polymers within and between plants using novel microarrays. Plant J. 2007;50(6):1118-28.

3. Crouch LI, Labourel A, Walton PH, Davies GJ, Gilbert HJ. The contribution of non-catalytic carbohydrate binding modules to the activity of lytic polysaccharide monooxygenases. J Biol Chem. 2016;291(14):7439-49.

4. Cantarel BL, Coutinho PM, Rancurel C, Bernard T, Lombard V, Henrissat B. The Carbohydrate-Active EnZymes database (CAZy): an expert resource for glycogenomics. Nucleic Acids Res. 2009;37(suppl_1):D233-D8. 5. McCartney L, Blake AW, Flint J, Bolam DN, Boraston AB, Gilbert HJ, et al. Differential recognition of plant cell walls by microbial xylan-specific carbohydrate-binding modules. Proc. Natl. Acad. Sci. U.S.A. 2006;103(12):4765-70.

6. Blake AW, McCartney L, Flint JE, Bolam DN, Boraston AB, Gilbert HJ, et al. Understanding the biological rationale for the diversity of cellulose-directed carbohydrate-binding modules in prokaryotic enzymes. $\mathrm{J}$ Biol Chem. 2006;281(39):29321-9.

7. Filonova L, Kallas ÅM, Greffe L, Johansson G, Teeri TT, Daniel G. Analysis of the surfaces of wood tissues and pulp fibers using carbohydrate-binding modules specific for crystalline cellulose and mannan. Biomacromolecules. 2007;8(1):91-7.

8. Knox JP. Revealing the structural and functional diversity of plant cell walls. Curr Opin Plant Biol. 2008;11(3):308-13.

9. Moller I, Marcus SE, Haeger A, Verhertbruggen Y, Verhoef R, Schols H, et al. High-throughput screening of monoclonal antibodies against plant cell wall glycans by hierarchical clustering of their carbohydrate microarray binding profiles. Glycoconj J. 2008;25(1):37-48.

10. Altaner C, Hapca AI, Knox JP, Jarvis MC. Detection of $\beta$-1-4-galactan in compression wood of Sitka spruce [Picea sitchensis (Bong.) Carrière] by immunofluorescence. Holz. 2007;61(3):311-6.

11. Clausen MH, Willats WG, Knox JP. Synthetic methyl hexagalacturonate hapten inhibitors of antihomogalacturonan monoclonal antibodies LM7, JIM5 and JIM7. Carbohydr Res. 2003;338(17):1797-800.

12. Willats WG, McCartney L, Steele-King CG, Marcus SE, Mort A, Huisman M, et al. A xylogalacturonan epitope is specifically associated with plant cell detachment. Planta. 2004;218(4):673-81.

13. McCartney L, Marcus SE, Knox JP. Monoclonal antibodies to plant cell wall xylans and arabinoxylans. J Histochem Cytochem. 2005;53(4):543-6.

14. Marcus SE, Verhertbruggen Y, Hervé C, Ordaz-Ortiz JJ, Farkas V, Pedersen HL, et al. Pectic homogalacturonan masks abundant sets of xyloglucan epitopes in plant cell walls. BMC Plant Biol. 2008;8(1):60.

15. Smallwood M, Yates EA, Willats WG, Martin H, Knox JP. Immunochemical comparison of membraneassociated and secreted arabinogalactan-proteins in rice and carrot. Planta. 1996;198(3):452-9.

16. Pattathil S, Avci U, Baldwin D, Swennes AG, McGill JA, Popper Z, et al. A comprehensive toolkit of plant cell wall glycan-directed monoclonal antibodies. Plant Physiol. 2010;153(2):514-25. 


\section{Supplemental Table:}

\begin{tabular}{|c|c|}
\hline CBMs & Specificity \\
\hline CBM2 & $\beta$-D-Glcp-(1-4)- $\beta$-D-Glcp-(1-4)- $\beta$-D-Glcp \\
\hline CBM3 & $\beta$-D-Glcp-(1-4)- $\beta$-D-Glcp \\
\hline \multirow[b]{4}{*}{ CBM6 } & $\beta$-D-Xylp-(1-4)- $\beta$-D-Xylp-(1-4)- $\beta$-D-Xylp \\
\hline & 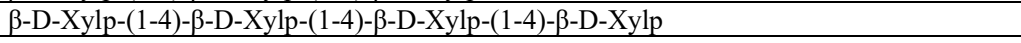 \\
\hline & $\beta$-D-Xylp-(1-4)- $\beta$-D-Xylp-(1-4)- $\beta$-D-Xylp \\
\hline & $\beta$-D-Glcp-(1-4)- $\beta$-D-Galp-(1-4)- $\beta$-D-Glcp-(1-4)- $\beta$-D-Glcp \\
\hline CBM4-9-1 & $\beta$-D-Glcp-(1-3)- $\beta$-D-Glcp-(1-3)- $\beta$-D-Glcp-(1-3)- $\beta$-D-Glcp-(1-3)- $\beta$-D-Glcp-(1-3)- $\beta$-D-Glcp \\
\hline CBM4-9-2 & $\beta$-D-Xylp-(1-4)- $\beta$-D-Xylp-(1-4)- $\beta$-D-Xylp-(1-4)- $\beta$-D-Xylp-(1-4)- $\beta$-D-Xylp \\
\hline CBM11 & Beta-1,4- and Beta-1,3-1,4-Mixed Linked Glucans at a Single Binding Site \\
\hline \multirow[b]{5}{*}{ CBM13 } & $\alpha$-D-Manp-(1-2)- $\alpha$-D-Manp \\
\hline & $\alpha$-D-Manp-(1-2)- $\alpha$-D-Manp \\
\hline & $\alpha$-D-Manp-(1-2)- $\alpha$-D-Manp \\
\hline & $\alpha$-D-Manp-(1-2)- $\alpha$-D-Manp \\
\hline & $\alpha$-D-Manp-(1-2)- $\alpha$-D-Manp-(1-2)- $\alpha$-D-Manp \\
\hline \multirow[b]{2}{*}{ CBM13-1 } & $\alpha$-L-Arap \\
\hline & $\beta$-D-Galp \\
\hline \multirow[b]{3}{*}{ CBM13-2 } & $\beta$-D-Galp-(1-4)- $\beta$-D-Glcp \\
\hline & $\beta$-D-Xylp-(1-4)- $\beta$-D-Xylp-(1-4)- $\beta$-D-Xylp-(1-4)- $\beta$-D-Xylp-(1-4)- $\alpha$-D-Xylp \\
\hline & 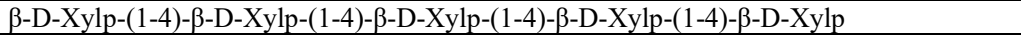 \\
\hline CBM13-3 & $\beta$-D-Galp-(1-4)- $\alpha-\mathrm{D}-\mathrm{Glcp}$ \\
\hline \multirow[b]{5}{*}{ CBM13-4 } & $\beta$-D-Galp-(1-3)- $\beta$-D-Galp \\
\hline & $\beta$-D-Galp-(1-3)- $\beta$-D-Galp-(1-3)- $\beta$-D-Galp \\
\hline & $\beta$-D-Galp-(1-4)- $\beta$-D-Glep \\
\hline & $\beta$-D-Galp \\
\hline & $\beta$-D-Galp1S-(1-1)-<non carb $>$ \\
\hline CBM15 & 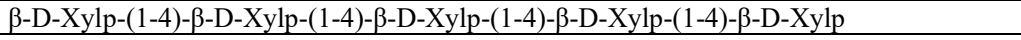 \\
\hline CBM17 & $\beta$-D-Glcp-(1-4)- $\beta$-D-Glcp-(1-4)- $\beta$-D-Glcp-(1-4)- $\beta$-D-Glcp \\
\hline \multirow[b]{4}{*}{ CBM20 } & $\alpha$-D-Glcp-(1-4)- $\alpha$-D-Glcp \\
\hline & $\begin{array}{l}\alpha \text {-D-Glcp-(1-4)- } \alpha \text {-D-Glcp-(1-4)- } \alpha \text {-D-Glcp1N-(1-4)- } \alpha \text {-D-6-deoxy-Glcp4N-(1-4)- } \alpha \text {-D-Glcp- } \\
(1-4)-\beta \text {-D-Glcp }\end{array}$ \\
\hline & $\alpha$-D-Glcp-(1-4)- $\alpha-\mathrm{D}-\mathrm{Glcp}$ \\
\hline & $\alpha$-D-Glcp-(1-4)- $\beta$-D-Glcp \\
\hline \multirow[b]{2}{*}{ CBM21 } & $\alpha$-D-Glcp-(1-6)- $\alpha$-D-Glcp-(1-6)- $\alpha$-D-Glcp-(1-6)- $\alpha$-D-Glcp \\
\hline & $\alpha$-D-Glcp-(1-6)- $\alpha$-D-Glcp-(1-6)- $\alpha$-D-Glcp \\
\hline \multirow[b]{2}{*}{ CBM26 } & $\alpha$-D-Glcp-(1-4)- $\alpha$-D-Glcp \\
\hline & $\beta$-D-Glcp \\
\hline \multirow{3}{*}{$\begin{array}{l}\text { CBM27 } \\
\text { CBM29- } \\
\text { CBM65-1 }\end{array}$} & $\beta$-D-Manp-(1-4)- $\beta$-D-Manp-(1-4)- $\beta$-D-Manp-(1-4)- $\beta$-D-Manp-(1-4)- $\beta$-D-Manp \\
\hline & $\begin{array}{l}\alpha \text {-D-Galp-(1-6)[ } \beta \text {-D-Manp-(1-4)]- } \beta \text {-D-Manp-(1-4)[ } \alpha-D-G a l p-(1-6)]-\beta-D-M a n p-(1-4)-\beta-D- \\
\text { Manp-(1-4)- } \beta \text {-D-Manp }\end{array}$ \\
\hline & \\
\hline \multirow{5}{*}{$\begin{array}{l}\text { CBM29- } \\
\text { CBM65-2 }\end{array}$} & $\begin{array}{l}\beta \text {-D-Manp-(1-4)- } \beta \text {-D-Manp-(1-4)- } \beta \text {-D-Manp-(1-4)- } \beta \text {-D-Manp-(1-4)- } \beta \text {-D-Manp-(1-4)- } \beta \text {-D- } \\
\text { Manp }\end{array}$ \\
\hline & $\beta$-D-Glcp-(1-4)- $\beta$-D-Glcp-(1-4)- $\beta$-D-Glcp-(1-4)- $\beta$-D-Glcp-(1-4)- $\beta$-D-Glcp-(1-4)- $\alpha$-D-Glcp \\
\hline & $\beta$-D-Glcp-(1-4)- $\beta$-D-Glcp-(1-4)- $\beta$-D-Glcp-(1-4)- $\beta$-D-Glcp-(1-4)- $\beta$-D-Glcp-(1-4)- $\alpha$-D-Glcp \\
\hline & $\beta$-D-Glcp-(1-4)- $\beta$-D-Glcp-(1-4)- $\beta$-D-Glcp-(1-4)- $\beta$-D-Glcp-(1-4)- $\beta$-D-Glcp-(1-4)- $\alpha$-D-Glcp \\
\hline & $\beta$-D-Manp-(1-4)- $\beta$-D-Manp-(1-4)- $\beta$-D-Manp-(1-4)- $\beta$-D-Manp-(1-4)- $\beta$-D-Manp \\
\hline \multirow[b]{3}{*}{ CBM35 } & $\alpha$-D-Glcp-(1-6)- $\beta$-D-Glcp \\
\hline & $\alpha$-D-Glcp-(1-6)- $\alpha$-D-Glcp \\
\hline & $\alpha$-D-Glcp-(1-6)- $\alpha-D-G l c p-(1-4)-\alpha-D-G l c p$ \\
\hline CBM36 & $\beta$-D-Xylp-(1-4)- $\beta$-D-Xylp-(1-1)-methyl \\
\hline CBM39 & $\beta$-D-Glcp-(1-3)- $\beta$-D-Glcp-(1-3)- $\beta$-D-Glcp-(1-3)- $\beta$-D-Glcp-(1-3)- $\beta$-D-Glcp-(1-3)- $\beta$-D-Glcp \\
\hline CBM41 & $\alpha$-D-Glcp-(1-4)- $\alpha$-D-Glcp \\
\hline
\end{tabular}




\begin{tabular}{|c|c|}
\hline \multicolumn{2}{|c|}{\begin{tabular}{l|l|} 
& $\alpha$-D-Glcp-(1-4)- $\alpha$-D-Glcp \\
\cline { 2 - 2 }
\end{tabular}} \\
\hline & $\alpha$-D-Glcp-(1-4)- $\alpha$-D-Glcp-(1-4)- $\alpha$-D-Glcp \\
\hline & $\alpha$-D-Glcp-(1-4)- $\alpha$-D-Glcp \\
\hline & 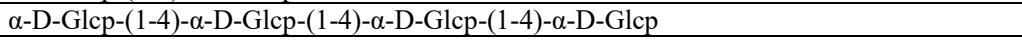 \\
\hline \multirow[t]{2}{*}{ CBM47 } & $\alpha$-D-Glcp-(1-3)- $\alpha$-D-Glcp \\
\hline & $\alpha$-D-Glcp-(1-4)- $\beta$-D-Glcp \\
\hline CBM51 & $\beta$-D-Galp-(1-1)-methyl \\
\hline \multirow[b]{3}{*}{ CBM58 } & $<$ non carb>-(1-4)- $\alpha$-D-6-deoxy-Glcp4N-(1-4)- $\alpha$-D-Glcp-(1-4)- $\alpha$-D-Glcp \\
\hline & $<$ non carb $>-(1-4)-\alpha$-D-6-deoxy-Glcp4N-(1-4)- $\beta$-D-Glcp-(1-4)-ASP \\
\hline & $\alpha$-D-Glcp-(1-4)- $\alpha$-D-Glcp \\
\hline \multirow[b]{2}{*}{ CBM60 } & $\beta$-D-Glcp-(1-4)- $\beta$-D-Glcp \\
\hline & $\beta$-D-Galp-(1-4)- $\beta$-D-Galp \\
\hline \multirow[b]{2}{*}{ CBM61 } & $\beta$-D-Galp-(1-4)- $\beta$-D-Galp-(1-4)- $\beta$-D-Galp \\
\hline & $\beta$-D-Galp-(1-4)- $\beta$-D-Galp-(1-4)- $\beta$-D-Galp \\
\hline CBM62 & $\alpha$-D-Galp-(1-6)- $\beta$-D-Manp \\
\hline \multirow[b]{2}{*}{ CBM63 } & 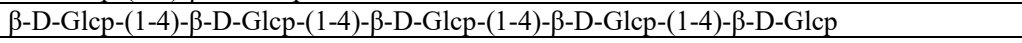 \\
\hline & $\beta$-D-Glcp-(1-4)- $\beta$-D-Glcp-(1-4)- $\beta$-D-Glcp-(1-4)- $\beta$-D-Glcp-(1-4)- $\beta$-D-Glcp-(1-4)- $\beta$-D-Glcp \\
\hline \multirow{2}{*}{ CBM66 } & $\beta$-D-Fruf \\
\hline & $\beta$-D-Fruf-(2-6)- $\beta$-D-Fruf \\
\hline CBM67 & $\alpha$-L-Rhap \\
\hline \multirow[b]{3}{*}{ CBM71 } & $\beta$-D-Galp-(1-4)- $\beta$-D-GlcpNAc \\
\hline & 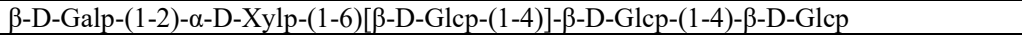 \\
\hline & $\alpha$-D-Galp-(1-6)- $\beta$-D-Manp \\
\hline \multirow[b]{5}{*}{ CBM80 } & $\beta$-D-Manp-(1-4)- $\beta$-D-Manp-(1-4)- $\beta$-D-Manp-(1-4)- $\beta$-D-Manp-(1-4)- $\beta$-D-Manp \\
\hline & $\begin{array}{l}\beta \text {-D-Manp-(1-4)- } \beta \text {-D-Manp-(1-4)- } \beta \text {-D-Manp-(1-4)- } \beta \text {-D-Manp-(1-4)- } \beta \text {-D-Manp-(1-4)- } \beta \text {-D- } \\
\text { Manp }\end{array}$ \\
\hline & $\beta$-D-Glcp-(1-4)- $\beta$-D-Glcp-(1-4)- $\beta$-D-Glcp \\
\hline & $\beta$-D-Manp-(1-4)- $\beta$-D-Manp-(1-4)- $\beta$-D-Manp \\
\hline & $\beta$-D-Manp-(1-4)- $\beta$-D-Manp-(1-4)- $\beta$-D-Manp-(1-4)- $\beta$-D-Manp-(1-4)- $\beta$-D-Manp \\
\hline \multirow[b]{2}{*}{ CBM81 } & $\beta$-D-Glcp-(1-4)- $\beta$-D-Glcp-(1-4)- $\beta$-D-Glcp-(1-4)- $\beta$-D-Glcp-(1-4)- $\beta$-D-Glcp \\
\hline & $\beta$-D-Glcp-(1-4)- $\beta$-D-Glcp-(1-4)- $\beta$-D-Glcp-(1-4)- $\beta$-D-Glcp-(1-4)- $\beta$-D-Glcp \\
\hline
\end{tabular}

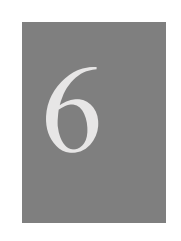


Table S2. Duplicate fluorescence values due to binding of CBM27-GFP fusion to polysaccharides when tested in the Glycoprofiling.

\begin{tabular}{|c|c|c|}
\hline & CBM27 & CBM27 \\
\hline Wheat arabinoxylan insoluble & 0 & 0 \\
\hline Wheat arabinoxylan acid debranched; $22 \%$ arabinose & 0 & 1 \\
\hline 1,4- $\beta$-D-mannan Brohydride reduced & 0 & 0 \\
\hline Mannan(Ivory nut) & 0 & 0 \\
\hline Wheat arabinoxylan low visosity & 0 & 0 \\
\hline Galactomannan(carob) low viscosity & 2 & 4 \\
\hline Debranched arabinan & 0 & 0 \\
\hline Azo-galacromannan from carob & 2 & 4 \\
\hline Lichenan (Icelandicmoss) 1,3:1,4- $\beta$-D-Glucan & 0 & 1 \\
\hline Rye arabinoxylan high viscosity & 0 & 0 \\
\hline Pullulan & 0 & 0 \\
\hline Guar medium visicosity xylan(beechwood) & 17 & 19 \\
\hline Karaya gum & 0 & 0 \\
\hline Rhamnogalacturonan from soy bean pectic fibre & 0 & 0 \\
\hline Larch arabinogalactan & 0 & 1 \\
\hline Galactomannan(carob) high viscosity & 14 & 12 \\
\hline Guar galactomannan(GD21) enzyme modified guar & 14 & 13 \\
\hline Guar galactomannan (GD 28) enzyme modified guar & 18 & 16 \\
\hline Arabinan sugar beet & 0 & 0 \\
\hline Pectic galactan potato & 0 & 0 \\
\hline Beta glucan(barley) low viscosity & 0 & 1 \\
\hline Linear 1,5- $\alpha$-L-arabian sugar beet & 0 & 1 \\
\hline Galactan & 0 & 1 \\
\hline Pachyman 1,3- $\beta$-D-glucan & 0 & 0 \\
\hline Xyloglucan from tamarind seed & 0 & 0 \\
\hline Cm-pachyman & 0 & 0 \\
\hline Rhamnogalaturonan from potato pectic fibre & 0 & 1 \\
\hline Cm-curdlan degree of carboxymethylation & 0 & 0 \\
\hline Amylose (potato & 0 & 0 \\
\hline Glucomannan(konjac) low viscosity & 5 & 5 \\
\hline Chitin from shrimp shells & 0 & 0 \\
\hline D-galacto-D-mannan from ceratonia & 21 & 20 \\
\hline Dextran & 0 & 0 \\
\hline Cm-cellulose 4M for endo-cellulase & 0 & 0 \\
\hline Xanthan gum from axnthomonas & 0 & 1 \\
\hline Tragacanth & 0 & 2 \\
\hline Gum Arabic & 0 & 0 \\
\hline 4-O-Methyl-D-glucurono-D-xylan & 0 & 0 \\
\hline Xylan beechwood & 0 & 0 \\
\hline Locust beangum from ceratonia siliqua seeds & 13 & 12 \\
\hline Beta glucan (oat) medium viscosity & 0 & 2 \\
\hline Negative control & 0 & 1 \\
\hline Positive control & 20 & 21 \\
\hline
\end{tabular}




\section{Chapter 7}

General discussion 


\subsection{Motivation and aim of the research}

The project titled "Improving intestinal degradation of recalcitrant fibres in pigs" aimed to improve the utilization of recalcitrant fibres in swine feed from by-products of seed oil industry, in order to reduce feed costs and maintain sustainable use of feed raw materials.

As fibres are fermented/utilized by the gut microbiota, a swine large intestinal in vitro model was developed to study the effect of fibres on swine gut microbiota. In this thesis, fibres in rapeseed meal (RSM), which is a cheap and abundant by-product of rapeseed oil production, were studied. The effect of three enzymatic treatments and one chemical treatment on the recalcitrant fibres of RSM was investigated. The effect of the unmodified and the four modified RSMs on the swine gut microbiota (composition and activity) was studied. Last, a preliminary novel high-throughput technique to screen polysaccharide composition and structure was developed.

\subsection{Swine in vitro Large Intestinal Model}

The Swine in vitro Large Intestinal Model (SLIM) was developed based on the human, computer-controlled, dynamic TNO in vitro model of colon, nick-named TIM-2 (1). As TIM2 was originally designed for human beings, the colon parameters, such as temperature $\left(37^{\circ} \mathrm{C}\right), \mathrm{pH}(5.8)$, and Standard Ileal Efflux Medium (SIEM), are adapted to the physiology of human beings. For SLIM, therefore temperature $\left(39{ }^{\circ} \mathrm{C}\right), \mathrm{pH}(5.9)$ and medium composition (SIEMP; SIEM for pigs) were modified to correspond to swine physiology (2, 3)

7.2.1 Similarities between the microbiota composition of the original pig fecal inoculum and the microbiota fed with modified SIEM containing different starch sources

After feeding the gut microbiota in SLIM modified SIEM contataining different starch sources (maize, potato or wheat), in search of the optimal starch source for the pig microbiota, the microbiota composition was determined and a network analysis was performed (Chapter 2). The different starch sources impacted the microbiota composition: they had a considerable effect on both alpha-diversity (inverse Simpson, and Faith's PD), and beta-diversity (weighted and unweighted UniFrac, and Bray-Curtis) (Chapter 2), indicating that some microbial species (composition and/or abundance) among the three starch treatments were significantly different. In Chapter 2, among the three starch treatments, 11 genera, 7 genera and 7 genera were more abundant upon maize, potato, and wheat starch treatment, respectively. The inherent properties of starches, such as crystallinity, granular structure, amylose:amylopectin ratio, and retrogradation of amylose, will influence the formation of resistant starch (4). Therefore, the proportion and amount of resistant starch types of the starch sources may have led to alteration of the microbiota composition after feeding these starches.

The ensemble method was used based on multiple similarity measures in combination with generalized boosted linear models on microbial abundance (5). This investigates whether the shift of microbial abundance will change the bacterial co-occurrence relationships. Our results showed that maize starch-based network had much better scale-free properties, as evaluated by the value of degree distribution fitting $\mathrm{R}^{2}$ (Chapter 2). Scale-free networks display a very strong robustness against random failures (6). Thus the microbiota fed with maize starch may be more stable or resilient, compared to those fed with potato and wheat starch.

In order to investigate which functional composition driven by the different starch sources was more similar to the original pig microbiota inoculum, a pig fecal sample was included in the analyses. Both the PCA and the heatmap of the functional predictive profile showed that 
the original pig fecal sample clustered with maize starch treatment, which indicated that the functional profile of these samples was more similar to the original pig inoculum (Chapter 2).

As discussed above, the microbiota fed with maize starch may be more stable or resilient, compared to those fed potato or wheat starch, which can more closely simulate the microbial ecology in vivo. And the predictive functional metagenome of the microbiota fed with maize starch was more similar to the original inoculum. Thus, maize starch was used as starch source in the recipe of standard ileal effluent medium for pigs (SIEMP). But the microbial composition between microbiota fed with maize starch and the original inoculum still was very different. The original inoculum separated in a principal coordinate analysis from the microbiota fed with one of the three starch sources, which indicated a different microbial composition between the original inoculum and starch-fed microbiotas (Chapter 2). This is reasonable, as pig diets have different carbohydrate sources than human beings. The dominant phyla in the maize starch-fed microbiota were Firmicutes and Actinobacteria, while they were Firmicutes and Bacteroidetes in the original inoculum (Chapter 2). Previous reports (7-9) have also shown that the most abundant phyla were Firmicutes and Bacteroidetes in pig gut microbiota. Therefore, the next study was performed, in which an attempt was made to adjust the microbial composition in the in vitro model to become more similar to the original inoculum.

7.2.2 Similarities between the microbiota composition of the original pig fecal inoculum and the microbiota fed with maize starch-based SIEMP supplemented with arabinoxylan (AX) or AX and xyloglucan (XG)

Compared to only supplementing AX to maize starch based SIEMP, microbiota fed AX-XG on top of maize-starch had a higher relative abundance of Bacteroidetes and Firmicutes, and a lower relative abundance of Actinobacteria, which made the microbial composition more similar to the original inoculum (Chapter 2). This indicated that the XG supplementation can further increase a relative abundance of bacteria in the phyla Bacteroidetes and Firmicutes, at the expense of the phylum Actinobacteria. Larsbrink et. al (10) confirmed Bacteroidetes can catabolize XG by a discrete genetic locus, which corroborates that XG supplementation induced the growth of Bacteroidetes in our study. Cantu-Jungles et. al (11) reported that a substantial increase in the $B$. uniformis population occurred after XG addition to an in vitro fecal fermentation. At a lower phylogenetic level in our study, XG mainly increased the amount of Prevotellaceae, and led to a slight decrease in the number of Atopobiaceae, Lachospiraceae, and Muribaculaceae. We did not find any research about Prevotellaceae catabolizing XG in pigs. But Svartström et. al (12) reported that Prevotellaceae from the rumen microbiota can express xyloglucanses, which are XG-degrading enzymes.

Supplementation with both $\mathrm{AX}$ and $\mathrm{XG}$ in maize-based SIEMP also showed higher reproducibility than only supplementing AX to maize-based SIEMP. Chapter 2 shows that microbiota compositions between replicates at different time points upon AX-XG feeding was much more similar than those on AX. In order to test the reproducibility of microbial composition, C.V. of replicates of microbiota fed with AX-XG of alpha diversity, microbial abundance, and microbial activities were evaluated (Chapter 2), which were all lower than $15 \%$ (expect for 5 taxa in microbial abundance). The low C.V.s indicated that the results of the biological replicates were very reproducible (13). The high reproducibility of TIM-2 (the human version) was previously evaluated by the clustering of the microbiota after the adaptation period (at t0) (14), and during an entire experiment (15). Since SLIM (or TIM-2) 
is computer-controlled, it is highly reproducible. This has been shown in numerus studies (15-18).

All data collectively considered, maize starch with arabinoxylans (AX) and xyloglucan (XG) should be added to the SIEM recipe to simulate ileal effluent medium for pigs (SIEMP), and this outcome layed the foundation for experiments mimicking the swine large intestine fermentation in the other chapters of this thesis.

\subsection{Characteristics of non-processed and processed RSM}

RSM was processed by a cellulase (CELL), two pectinase (PECT1 and PECT2), alkaline (ALK) treatment or nothing (CON) by two different method (Figure 1). Method I, RSM was processed before predigesting its protein; Method II, RSM was processed after prediesting its protein (Figure 1). Moreover, biological (Chapter 3) and chemical method (Chapter 5) were performed to charactise polysaccharide composition of non-processed and processed RSM.

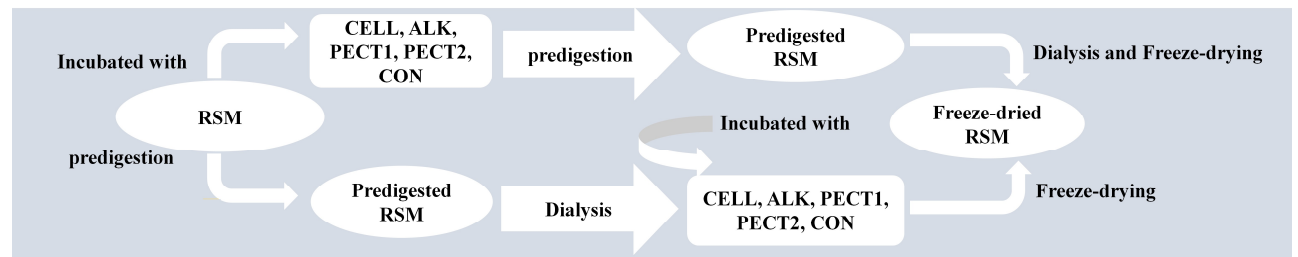

Figure 1. Schematic representation of processing RSM by two different method.

\subsubsection{Evaluation by glycoprofiling with monoclonal antibodies (mAbs)}

Cell wall polysaccharides of RSM (Brassica napus) were comprehensively studied by chemical methods (19), which showed that they generally consisted of arabinan, homogalacturonan, Rhamnogalacturoan I (RG-I), type II arabinogalactan (AG), and xyloglucan (XG) (20). In a previous study (Long, de Vries and Venema submitted), we determined the monosaccharide constituent composition, which was in line with Pustjens et al. (2013). Our current findings, using glycoprofiling with the mAbs, are also in line with this, since nonfucosylated XG-, RG-I-, pectic backbone-, and AG-recognizing mAbs bound to CON. By similar reasoning, xylan was also detected in $\mathrm{CON}$, which was not reported before, although it is not entirely clear how much cross-reactivity the mAbs show.

ALK treatment intensively increased binding of mAbs that specifically bind to nonfucosylated XG (Chapter 3). This is consistent with previous finding that alkali could extract XG from hemicellulose (19), which led to XG being detected by the mAbs in the current study. Previous research also demonstrated that XG is linked via hydrogen bridges to the surface of cellulose microfibrils, which is extractable with alkali, but not accessible by enzyme (21). However, enzymatic treatments (PECT1, PECT2, and CELL) led to a disappearance of binding nonfucosylated XG-specific mAbs compared to CON, which was unexpected. It could be that XG might become entrapped by other cell wall structures after pectinase or cellulase treatment. It is unknown whether this kind of the entrapment phenomenon would reduce the degradability of XG.

Enzymatic treatments increased binding of linseed mucilage RG-I-specific mAbs and some mAbs directed against the arabinogalactan side chains of RG-I (RG-I/AG), and reduced binding of some mAbs directed to pectic backbone, which indicated that PECT1 and PECT2 broke down pectic backbone and exposed RG-I and its arabinogalactan side chains. These results are supported by a previous study showing that PECT1 and PECT2 degraded pectic 
backbone and released RG-I and its arabinogalactan side chains (19). Moreover, RG-I attached to cellulose microfibrils can be released by cellulase (22), while at the same time it might block the accessibility of pectic backbone because of the shifting of the polysaccharides structure.

ALK treatment led to the disappearance of binding of mAbs that specifically bind to AG-1 while it increased binding of mAbs that specifically binding to AG-2, which indicated that ALK could cause arabinogalactan to be physically entrapped in other cell wall structures, while linear and branched arabinan become accessible (23).

They binding strengths of mAbs in AG-4 were lower upon ALK_B, PECT1_B, PECT2_B, and CELL_B treatment, compared to ALK A, PECT1_A, PECT'2_A, and CELL_A. This indicated that small fragments were produced in AG-4 group after enzymatic and chemical treating RSM, which were dialyzed out in ALK_B, PECT1_B, PECT2_B, and CELL_B during the subsequent predigestion treatment. Overall, both enzymatic and chemical processed RSM can release some polysaccharides but physically entrap or shield some others at the same time.

\subsubsection{Evaluation by chemical method}

The carbohydrate content of $\mathrm{CON}$ is $62 \% \mathrm{w} / \mathrm{w}$ (Chapter 5). Dominant sugars are glucose (Glc, $31 \mathrm{~mol} \%$ ), uronic acid (UA, $19 \mathrm{~mol} \%$ ), galactose (Gal, $11 \mathrm{~mol} \%$ ) and arabinose (Ara, $5 \mathrm{~mol} \%$ ). This is inline with privious study (19). CON contained $56 \%$ pectin (defined as: rhamnose + arabinose + galactose + uronic acid) and $44 \%$ (hemi)cellulose (xylose + mannose + fucose + glucose).

The values of pectin and (hemi)cellulose for ALK_A, ALK_B, PECT1_A, PECT1_B, PECT2_A, PECT2_B, CELL_A, and CELL_B were $\overline{55} \%$ and $\overline{45} \%, 52 \%$ and $48 \%, 5 \overline{4} \%$ and $46 \%, 50 \%$ and $50 \%, 56 \%$ and $44 \%, 51 \%$ and $49 \%, 57 \%$ and $43 \%$, and $60 \%$ and $40 \%$, respectively. Relative pectin contents decreased in ALK, PECT1, and PECT2 treatment, as expected for the PECT enzymes, while they increased in CELL treatment, compared to CON. PECT1 and PECT2 did not decrease pectin to a large extent, which might be caused by shielding or inaccessibility of the pectin molucules by (hemi)cellulose.

The ALK, PECT1, and PECT2 treatments increased (hemi)cellulose values, compared to $\mathrm{CON}$, as expected. Predigesting before or after processing RSM had little effect on the monosaccharide constitution.

7.4 The effect of (processed) RSM on the microbiota composition and fibre degradability in the SLIM

In SLIM, the swine microbiota was fed with non-processed and processed RSM by a single shot of $5 \mathrm{~g}$ RSM (Chapter 3, Figure 2A), continuously $48 \mathrm{~h}$ feeding of RSM (Chapter 4, Figure $2 \mathrm{~B}$ ), or a single shot of $5 \mathrm{~g}$ RSM after $48 \mathrm{~h}$ of adaptation to RSM (Chapter 5, Figure $2 \mathrm{C}$ ). In order to investigate the effect of processed RSM on the microbiota, $\beta$-diversity and relative abundance of microbial genera and predictive microbial function profile were analyzed. 


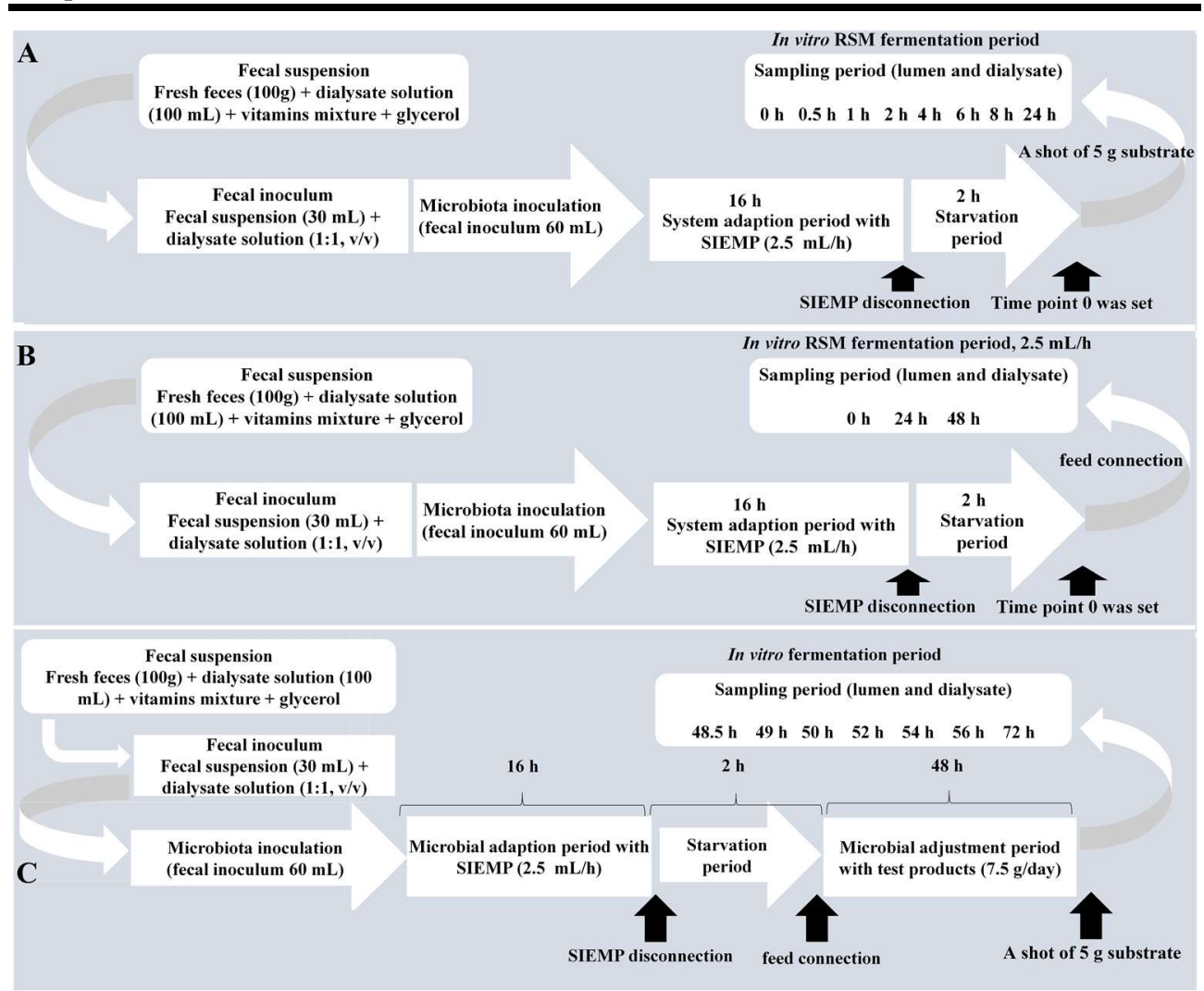

Figure 2. Schematic experimental setup for fibre fermentation in the Swine Large Intestine in vitro model (SLIM), in Chpater 3 (A), Chapter 4 (B) and Chapter 5 (C).

\subsubsection{Gut microbiota}

Our studies suggested that processed RSM (ALK, PECT1, PECT2 and CELL) had a significant different microbioal community compared to $\mathrm{CON}$ as evaluated by unweighted UniFrac, no matter how the RSM was fed (Chapter 3, 4 and 5). Dietary fibre is known to have a considerable effect on gut microbiota composition (24-26). Characterization of nonprocessed and processed RSM showed that polysaccharide structures were differentially shifted due to ALK, PECT1, PECT2, and CELL treatment in Chapter 3 and 5, which were consistent with previous studies $(27,28)$. These observations might explain the specific genus changes in microbiota composition after microbiotas were fed with ALK, PECT1, PECT2, and CELL compare to CON (Chapter 3, 4 and 5), despite the differential effect of the treatments on RSM as assessed by biological and chemical method (Chapter 3 and 5).

In order to understand which microbes were significantly influenced by dietary supplementation of processed RSM, microbial genera determined by next generation sequencing (V3-V4 region of the $16 \mathrm{~S}$ rDNA gene) were compared among the five treatments. In Chapter 3 (Figure 2A), the relative abundances of Ruminococcaceae NK4A214 group, Ruminococcaceae UCG-002, Ruminococcaceae UCG-005, Roseburia, Bifidobacterium, and Christensenellaceae R-7 group were significantly increased in ALK, PECT1, PECT2, and CELL. Research has shown that genera of the family Ruminococcaceae contain major (hemi)cellulolytic and pectinolytic species (29-31). Thus, microbes from the family 
Ruminococcaceae play an important role in degrading (hemi)cellulose and pectin in their activity against recalcitrant fibre $(32,33)$. Previous research demonstrated that the higher the relative abundance of Ruminococcaceae $N K 4 A 214$ group, the higher the fibre degradability $(30,34)$. This observation was consistent with the current study, which showed that the SCFA production (which is usually used as evaluation of fibre degradability in vitro) was also higher in ALK, CELL, PECT1, and PECT2 compared to CON. Furthermore, our study also showed that the relative abundance of Ruminococcaceae $N K 4 A 214$ group, as well as Ruminococcaceae UCG-002 and Ruminococcaceae UCG-005, significantly correlated with acetic acid, propionic acid, butyric acid, and (not surprisingly) total SCFA production. Roseburia is a well-known butyrate-producing bacteria (35), and a primary degrader of $\beta$ mannans (36). The significant increase in abundance of this genus was accordance with the increased butyric acid production in Chapter 3, and increased predicted abundance of betamannosidases (EC.3.2.1.25) in the processed RSM groups. Bifidobacterium is suggested to be a common cross-feeding bacteria for sugar utilization (37). Previous research has been shown that Bifidobacterium bifidum relies on the presence of a primary degrader in order to grow with either resistant starch or xylan both in vitro (38) and in vivo (39). It has also been reported that numerous Bifidobacterium species grew to higher cell densities accompanied by upregulating their respective saccharolytic pathways when grown in co-culture compared to their growth in monoculture (40). This finding was consistent with the current study, where the relative abundance of Bifidobacterium was much higher than that of other genera, especially in ALK. Alternatively, synergy existed among genera that were targeting the same substrates, possibly by specializing in degrading different motifs within the fibre molecule.

The relative abundance of Christensenellaceae $R-7$ group increased after enzymatic and chemical treatment, which was consistent with a previous study where rumen microbiota was fed with fibolytic enzyme-treated wheat straw (41). Christensenellaceae plays an important role in degrading fibre (42) and producing acetic and butyric acid (43). All the observations above were supported by former reports that feed processed by carbohydrase enzymes could stimulate growth of specific microbes (44-46). It can be speculated that the pretreatment of feed stuffs with carbohydrases causes the release of hydrolysis materials (presumably oligosaccharides), which promote the chemotactic response of specific bacteria, and stimulates their attachment to feed particles, thereby leading to growth of these microbes (45, 47-49).

In the Chapter 5 (Figure 2C), CELL treatment of RSM significantly increased the numbers of Olsenella, [Eubacterium] nodatum group, Acidaminococcus, Lachnospiraceae NK3A20 group, Bifidobacterium, Acetitomaculum, and Syntrophococcus. These genera may prefer to utilize cellulose- and/or hemicellulose-fragments generated by the action of CELL. However, in the literature only Eubacterium has been reported as cellulolytic (50). The CELL enzyme preparation contains multiple glycolytic activities, including exo-1,4- $\beta$-glucanase (cellobiohydrolase), endo-1,4- $\beta$-glucanase, hemicellulase and $\beta$-glucosidase. CELL may have broken down some bonds in cellulose thereby enhancing (hemi)cellulose utilization by the gut bacteria and at the same time also expose other polysaccharides (e.g. pectins) to other bacteria. ALK increased Megasphaera, and Ruminococcus gauvreauii group. Reports show that Ruminococcus are the most commonly found cellulolytic organisms (32, 50-52). Megasphaera is reported to contain glycosyl hydrolase (GH) family 53, which is involved in plant cell wall degradation (53). These reports together with our results indicate that ALK can disrupt the cell wall architecture, by solubilization of polysaccharides, presumably by breaking hydrogen bonds and hydrolyzing ester linkages thereby removing esters present as 
decoration on polysaccharides, making them more accessible for further enzyme degradation and utilization by the microbiota. Interestingly, both PECT1 and PECT2 showed no significant difference in Shannon index compared to CON and a very similar microbiota composition, which seemed to indicate that neither pectinase really changed the cell wall structure, or the changes did not meet the swine gut microbiotas' hydrolytic capacities, and as a result no bacteria were selectively stimulated. We reported before that a cocktail of PECT1 and PECT2 improved degradability of non-glucose polysaccharides of RSM in broilers $(27,28)$. Nevertheless, it seems that cell wall break down by PECT1 and PECT2 does not lead to advantages for members of the swine gut microbiota.

\subsubsection{Fibre degradation}

To investigate the dynamic changes of polysaccharide composition and structure in CON, ALK, CELL, PECT1, and PECT2 treated RSM during in vitro fermentation, a time series of sampling was performed and the set of $34 \mathrm{mAbs}$ was used to screen the lumen digests. Glycome profiling showed that binding of mAbs to lumen digests were dynamic during the in vitro fermentation in SLIM (Chapter 3 ). No bindings of mAbs recognizing non-fucolysated XG were observed in lumen samples after the microbiota was fed with ALK or CON (Chapter 3 ), which was unexpected as binding signals were seen in the substrates themselves prior to addition to SLIM (Chapter 3). The hypothesis could be entertained that XG was immediately utilized by bacteria before our first sampling time point (after 30 minutes), or that the XG structures were unable to be recognized by the mAbs after supplementing them to lumen, due to entrapment by other molecules. Binding signals still existed at 24 for all mAbs that showed signal at the 0.5 time point, which indicated that these structures cannot be degraded any further or more fermentation time was needed. Glycome profiling showed that binding of mAbs recognizing each polysaccharide structure/epitope were dynamic during the $24 \mathrm{~h}$ fermentation period (Chapter 3). It is not unlikely that certain polysaccharide structure, such as (hemi)cellulose and pectin, were exposed to microbes stage by stage, due to ever increasing degradation of the cell wall structures over time. For instance, bacteria should break down side-chain AG before they can utilize the backbone of RG-I. However, this hypothesis should be validated in future studies.

The chemical analytical method (Chapter 5) showed that the amount of main monosaccharides (arabinose, galactose, glucose, and uronic acid) was lower after $24 \mathrm{~h}$ fermentation for ALK and CELL compared to CON. The utilization of the main monosaccharides plateaued after time point 52 (4 hours after the shot), except for glucose, which was continuously utilized until time point 72 (24 hours after the shot). The utilization of arabinose and galactose was faster in ALK and PECT1, compared to the other treatments. HPSEC showed that an almost complete disappearance in the high Mw fraction occurred from $4 \mathrm{~h}$ onward (at time point $52 \mathrm{~h}$ ), indicating degradation and/or utilization of all high Mw polysaccharides. Compared to the highest peak of the elution profile of CON (48.5 h), the highest peaks of the elution profile of ALK and CELL (50 h and $49 \mathrm{~h}$, respectively) showed a higher amount of high Mw material, while those of the elution profile of PECT1 and PECT2 were approximately equal to CON (Chapter 5). This indicated that more materials were solubilized under the conditions when ALK and CELL-treated RSM were fed, than with the other substrates.

SCFA production was used to evaluate the fibre degradability of RSM by the swine gut microbiota. The cumulative acetic, propionic, and butyric acid and total SCFA production were higher in ALK, CELL, PECT1, and PECT2 compared to CON after the single shot (Chapter 3; Figure 2A). This observation was consistent with the predicted functional profiles 
related to carbohydrate metabolism, where the relative abundance of fibre breakdown and fermentation enzymes and pathways increased (Chapter 3). This finding was in accordance with previous studies that addition of pectolytic enzymes improved degradability of nonstarch polysaccharides (NSP) of RSM in vitro (27) and in broilers $(28,54)$. Giraldo et al. also reported that supplementation of exogenous cellulase increased SCFA production (45). Previous research demonstrated that supplementation of carbohydrase to feed before feeding could increase microbial protein production, ruminal cellulolytic bacterial numbers, and ruminal fibrolytic activity (44). Thus, the findings above indicate that the enzymatic and chemical treatment on RSM could sufficiently open cell wall architecture, to enable effective accessibility of NSP to bacterial degradation enzymes, and subsequently stimulate expression of microbial saccharolytic pathways.

In Chapter 4 (Figure 2B), there were no significant differences in SCFA production between unprocessed and processed RSM during the fibre fermentation period. This indicated that the processing applied to RSM could not improve their degradability compared to CON, in other words, they might not increase the feed efficiency during this period in vivo. It might be the fibre adaptation period was not enough to express the maximum enzymatic potential (55). Alternatively, the $48 \mathrm{~h}$ fibre adaptation period was sufficient for the microbiota to allow it to adapt to the substrate and even ferment CON efficiently.

The amount of propionic acid and the total SCFA in ALK and CELL were significant higher than those in CON, while there were no significant differences in SCFA production in PECT1 and PECT2 compared with CON in Chapter 5 (Figure 2C). This was corroborated by the carbohydrate related microbial functional metabolism pathways, which showd that the abundances of pyruvate metabolism, propanoate metabolism, pentose phosphate pathway, galactose metabolism, energy metabolism, fructose and mannose metabolism, and carbohydrate digestion and absorption were higher in CELL and/or ALK compared to CON. The mobile nylon bag technique was used to validate the fibre degradability in ileal cannulated pigs. The results showed that more fibres were degraded in case of ALK and CELL treatment, compared to CON, while there was no increased fibre degradability in PECT1 and PECT2 treated RSM, compared to CON (Chapter 5).

All data collectively considered, different feed methods (Figure 2A-C) lead to different SCFA production in terms of ALK, CELL, CON, PECT1, and PECT2 (Chapter 3, 4, and 5). A relative long adaptation period is needed after a diet change to RSM for swine microbiota (Chapter 4). Therefore, a gradual feeding of (modified) RSM to the swine gut microbiota might lead to better RSM adaptation. ALK and CELL treatment on RSM can improve its fibre degradability (Chapter 5).

7.5 A preliminary novel high-throughput technique to screen cell wall polysaccharide structures

The study was perfomed to develop a methodology based on carbohydrate binding modules (CBM), which enables to detect and determine plant cell wall structures in plant materials. The key steps of the Glycoprofiling technique can be divided into three stages: polysaccharide immobilization, CBM recognition and CB,-GFP fusion protein binding, and fluorescence reading. The optimized type of 96-well plate was selected based on literature (23), where the polysaccharides are immobilized well on the surface of the bottom of the plate. The results showed high reproducibility and specificity in recognizing polysaccharides by the Glycoprofiling technique. The set of different GFP-CBM fusion proteins can be extended by adding different CBMs, as well as exchanging GFP for other colored-fluorescent proteins. This would ultimately lead to a suit of fluorescent fusion proteins to dissect the 
structure of dietary carbohydrates.

\subsection{Future perspectives}

The current studies were focused on investigating the effect of modified (enzymatic and chemical treatment) RSM on swine gut microbiota composition and activity, and the fibre degradability by the gut microbiota, with the aim to increase use of recalcitrant fibres by the pig gut microbiota. Our in vivo study validated that CELL and ALK rather than PECT1 or PECT2 increased fibre degradation in RSM, which could help to guide feed additive strategies to improve efficiency and productivity in swine industry. The current studies gave insight into how feed enzymes may modulate microbial status, which provides good opportunity to develop novel carbohydrase, particularly in swine feed.

It is complicated and costly to investigate the effect of feed fermentation by the gut microbiota in pigs in vivo, due to the interaction with other feed-components in the swine feed (such as other fibers), the ethical considerations (such as using pathogens, toxic compounds, and experiments in animal themselves) and other uncontrollable factors (such as infectious disease or death during the experiment, which are important for reproducibility of results). Therefore, the newly developed SLIM (Chapter 2) is an excellent alternative to study the effect on the composition and/or activity of the gut microbiota without ethical constraints. It allows the screening of a large number of substances such as dietary ingredients and drugs, and even would allow the use of radioactive compounds or pathogens.

The microbes modulated by (processed) RSM, which had significant positive correlations with SCFA production, might be potential SCFA producer or RSM consumer (Chapter 3, 4 and 5). It would be interesting to investigate or validate the role of the potential SCFA producer in improving recalcitrant fibre degradability in a future study. One possible way is to deep mine the genomes of theses microbes for encoded the carbohydrases by bioinformatic tools. What is more, these enzymes can be overexpressed in an expression host and subsequenctly used to process RSM to investigate its degradability in pigs. Our study also showed that some carbohydrate structures (e.g. pectin) could not be accessed by the corresponding enzymes (e.g. pectinase), which is the limit step of improving its degradability. New bioinformatic tools should be developed to explore this kind of new carbohydrase (which can access and degrade the inaccesibable structures), which might only exist in specific strains, strains that are increased by these recalcitrant fibres.

The microbes, which significantly negatively correlated with bindings of mAbs, might be the mAbs-specific polysaccharide-epitope degraders (Chapter 3). However, we still do not know fully how the enzymatic and chemical treatment on the RSM cell wall structure affect the bacterial utilization. Thus, known polysaccharide structures, either changed by modification by the feed/microbial enzymes or not, should be fed to the swine gut microbiota to study the limiting step of the utilization of the recalcitrant RSM fibres, in order to develop novel feed enzymes and to determine structure(fibre)-function(microbiota) relationships and the correlation to health. The latter (health status) of course cannot be tested in SLIM, and requires well-controlled animal studies. To truely be able to make such structure-function relationships, more powerful and high-throughput techniques should be developed to analyze the plant cell wall polysaccharides, also dynamically during microbial breakdown. Our glycoprofiling method (Chapter 6) is a promising high-throughput method to screen polysaccharide structures, however, it is still in its preliminary stage, and one has to extend the CBM toolkit. Furthermore, isolation of microbial enzymes, which can significantly utilize the recalcitrant fibre, as eluded to above, might be another way to develop novel cell wall degrading enzymes. 
From Chapter 3, PECT1 and PECT2 can improve SCFA production and only ALK and CELL increased fibre degradability as validated in Chapter 5. Therefore, combined addition of pectinase and cellulase/alkaline treatment might further improve the fibre fermentability of RSM. Chapter 4 demonstrated that a relative long adaptation period is needed after a diet change to RSM for the swine microbiota, which has to be considered in practice, in order to improve its fibre utilization.

In conclusion, this thesis showed the initital steps for enzymatic and chemical treatment of RSM to significantly improve its fibre fermentability(in vitro and in vivo) and change microbiota composition in vitro. The combination of both pectinase and cellulase is expected to further improve recalcitrant fibre degradability. The potential SCFA producers and polysaccharide degraders in RSM utilization might be worthy of studying further for their importance in improving RSM fibre fermentability. 


\section{References:}

1. Minekus M, Smeets-Peeters M, Bernalier A, Marol-Bonnin S, Havenaar R, Marteau P, et al. A computercontrolled system to simulate conditions of the large intestine with peristaltic mixing, water absorption and absorption of fermentation products. Appl Microbiol Biotechnol. 1999;53(1):108-14.

2. Pluske J, Hampson D. Rice-based Diets in Pigs: For Protection Against Intestinal Bacterial Infections: a Report for the Rural Industries Research and Development Corporation: Rural Industries Research and Development Corporation; 2005.

3. Jackson PG, Cockcroft PD. Handbook of pig medicine: Elsevier Health Sciences; 2007.

4. Sajilata MG, Singhal RS, Kulkarni PR. Resistant starch-a review. Comprehensive reviews in food science and food safety. Compr. Rev. Food Sci. Food Saf. 2006;5(1):1-17.

5. Faust K, Raes J. Microbial interactions: from networks to models. Nat. Rev. Microbiol. 2012;10:538.

6. Cohen R, Havlin S. Complex networks: structure, robustness and function: Cambridge university press; 2010 .

7. Ramayo-Caldas Y, Mach N, Lepage P, Levenez F, Denis C, Lemonnier G, et al. Phylogenetic network analysis applied to pig gut microbiota identifies an ecosystem structure linked with growth traits. The ISME journal. 2016;10(12):2973.

8. Casellas NM, Berri EM, Fabrellas JE, Levenez F, Lemonnier G, Denis C, et al., editors. Establishment of the swine gut microbiome during early life and further impact on adult health and performance. 34 International Society for Animal Genetics Conference; 2014.

9. Costa MO, Chaban B, Harding JCS, Hill JE. Characterization of the fecal microbiota of pigs before and after inoculation with "Brachyspira hampsonii". PLoS One. 2014;9(8):e106399.

10. Larsbrink J, Rogers TE, Hemsworth GR, McKee LS, Tauzin AS, Spadiut O, et al. Adiscrete genetic locus confers xyloglucan metabolism in select human gut Bacteroidetes. Nature. 2014;506(7489):498-502.

11. Cantu-Jungles TM, do Nascimento GE, Zhang X, Iacomini M, Cordeiro LM, Hamaker BR. Soluble xyloglucan generates bigger bacterial community shifts than pectic polymers during in vitro fecal fermentation. Carbohydr. Polym. 2019;206:389-95.

12. Svartström O, Alneberg J, Terrapon N, Lombard V, de Bruijn I, Malmsten J, et al. Ninety-nine de novo assembled genomes from the moose (Alces alces) rumen microbiome provide new insights into microbial plant biomass degradation. The ISME journal. 2017;11(11):2538.

13. Gomez KA, Gomez AA. Statistical procedures for agricultural research: John Wiley \& Sons; 1984.

14. Verhoeckx K, Cotter P, López-Expósito I, Kleiveland C, Lea T, Mackie A, et al. The impact of food bioactives on health: in vitro and ex vivo models: Springer; 2015.

15. Aguirre M, de Souza CB, Venema K. The gut microbiota from lean and obese subjects contribute differently to the fermentation of arabinogalactan and inulin. PloS one. 2016;11(7):e0159236.

16. Kovatcheva-Datchary P, Egert M, Maathuis A, Rajilić-Stojanović M, De Graaf AA, Smidt H, et al. Linking phylogenetic identities of bacteria to starch fermentation in an in vitro model of the large intestine by RNAbased stable isotope probing. Environ Microbiol. 2009;11(4):914-26.

17. Martinez RC, Cardarelli HR, Borst W, Albrecht S, Schols H, Gutiérrez OP, et al. Effect of galactooligosaccharides and Bifidobacterium animalis Bb-12 on growth of Lactobacillus amylovorus DSM 16698, microbial community structure, and metabolite production in an in vitro colonic model set up with human or pig microbiota. FEMS Microbiol Ecol. 2013;84(1):110-23.

18. Rose DJ, Venema K, Keshavarzian A, Hamaker BR. Starch-entrapped microspheres show a beneficial fermentation profile and decrease in potentially harmful bacteria during in vitro fermentation in faecal microbiota obtained from patients with inflammatory bowel disease. Br. J. Nutr. 2010;103(10):1514-24.

19. Pustjens AM, Schols HA, Kabel MA, Gruppen H. Characterisation of cell wall polysaccharides from rapeseed (Brassica napus) meal. Carbohydr Polym. 2013;98(2):1650-6.

20. Siddiqui IR, Wood PJ. Carbohydrates of rapeseed: a review. J. Sci. Food Agric. 1977;28(6):530-8.

21. Pauly M, Albersheim P, Darvill A, York WS. Molecular domains of the cellulose/xyloglucan network in the cell walls of higher plants. P1J. 1999;20(6):629-39.

22. Oechslin R, Lutz MV, Amadò R. Pectic substances isolated from apple cellulosic residue: structural characterisation of a new type of rhamnogalacturonan I. Carbohydr. Polym. 2003;51(3):301-10.

23. Pattathil S, Avci U, Baldwin D, Swennes AG, McGill JA, Popper Z, et al. A comprehensive toolkit of plant cell wall glycan-directed monoclonal antibodies. Plant Physiol. 2010;153(2):514-25.

24. Yatsunenko T, Rey FE, Manary MJ, Trehan I, Dominguez-Bello MG, Contreras M, et al. Human gut microbiome viewed across age and geography. nature. 2012;486(7402):222-7.

25. Cotillard A, Kennedy SP, Kong LC, Prifti E, Pons N, Le Chatelier E, et al. Dietary intervention impact on gut microbial gene richness. Nature. 2013;500(7464):585-8.

26. Flint HJ. The impact of nutrition on the human microbiome. Nutr Rev. 2012;70(suppl 1):S10-S3.

27. Pustjens AM, de Vries S, Gerrits WJ, Kabel MA, Schols HA, Gruppen H. Residual carbohydrates from 
in vitro digested processed rapeseed ( Brassica napus ) meal. J Agric Food Chem. 2012;60(34):8257-63.

28. De Vries S, Pustjens A, Kabel M, Kwakkel R, Gerrits W. Effects of processing technologies and pectolytic enzymes on degradability of nonstarch polysaccharides from rapeseed meal in broilers. Poult Sci. 2014;93(3):589-98.

29. Nyonyo T, Shinkai T, Mitsumori M. Improved culturability of cellulolytic rumen bacteria and phylogenetic diversity of culturable cellulolytic and xylanolytic bacteria newly isolated from the bovine rumen. FEMS Microbiol Ecol. 2014;88(3):528-37.

30. Hartinger T, Edwards JE, Gómez Expósito R, Smidt H, ter Braak CJ, Gresner N, et al. Differently pretreated alfalfa silages affect the in vitro ruminal microbiota composition. Front Microbiol. 2019;10:2761.

31. Pettipher GL, Latham MJ. Production of enzymes degrading plant cell walls and fermentation of cellobiose by Ruminococcus flavefaciens in batch and continuous culture. Microbiology. 1979;110(1):29-38.

32. Flint HJ, Bayer EA, Rincon MT, Lamed R, White BA. Polysaccharide utilization by gut bacteria: potential for new insights from genomic analysis. Nat. Rev. Microbiol. 2008;6(2):121-31.

33. Shinkai T, Kobayashi Y. Localization of ruminal cellulolytic bacteria on plant fibrous materials as determined by fluorescence in situ hybridization and real-time PCR. Appl Environ Microbiol. 2007;73(5):1646-52.

34. Hartinger T, Gresner N, Südekum K-H. In vitro ruminal fermentation characteristics of alfalfa silages in response to different pre-ensiling treatments. Anim. Feed Sci. Technol. 2019;258:114306.

35. Duncan SH, Hold GL, Barcenilla A, Stewart CS, Flint HJ. Roseburia intestinalis sp. nov., a novel saccharolytic, butyrate-producing bacterium from human faeces. Int J Syst Evol Microbiol. 2002;52(5):1615-20

36. La Rosa SL, Leth ML, Michalak L, Hansen ME, Pudlo NA, Glowacki R, et al. The human gut Firmicute Roseburia intestinalis is a primary degrader of dietary $\beta$-mannans. Nat. Commun. 2019;10(1):1-14.

37. Cockburn DW, Koropatkin NM. Polysaccharide degradation by the intestinal microbiota and its influence on human health and disease. J Mol Biol. 2016;428(16):3230-52.

38. Turroni F, Özcan E, Milani C, Mancabelli L, Viappiani A, van Sinderen D, et al. Glycan cross-feeding activities between bifidobacteria under in vitro conditions. Front Microbiol. 2015;6:1030.

39. Turroni F, Milani C, Duranti S, Mancabelli L, Mangifesta M, Viappiani A, et al. Deciphering bifidobacterial-mediated metabolic interactions and their impact on gut microbiota by a multi-omics approach. The ISME journal. 2016;10(7):1656-68.

40. Milani C, Lugli GA, Duranti S, Turroni F, Mancabelli L, Ferrario C, et al. Bifidobacteria exhibit social behavior through carbohydrate resource sharing in the gut. Sci Rep. 2015;5:15782.

41. Ribeiro GO, Gruninger RJ, Jones DR, Beauchemin KA, Yang WZ, Wang Y, et al. Effect of ammonia fiber expansion-treated wheat straw and a recombinant fibrolytic enzyme on rumen microbiota and fermentation parameters, total tract digestibility, and performance of lambs. J. Anim. Sci. 2020;98(5):skaa116.

42. Mao S, Zhang M, Liu J, Zhu W. Characterising the bacterial microbiota across the gastrointestinal tracts of dairy cattle: membership and potential function. Sci Rep. 2015;5:16116.

43. Morotomi M, Nagai F, Watanabe Y. Description of Christensenella minuta gen. nov., sp. nov., isolated from human faeces, which forms a distinct branch in the order Clostridiales, and proposal of Christensenellaceae fam. nov. Int J Syst Evol Microbiol. 2012;62(1):144-9.

44. Wang Y, McAllister TA, Rode LM, Beauchemin KA, Morgavi DP, Nsereko VL, et al. Effects of an exogenous enzyme preparation on microbial protein synthesis, enzyme activity and attachment to feed in the Rumen Simulation Technique (Rusitec). Br J Nutr. 2001;85(3):325-32.

45. Giraldo L, Tejido M, Ranilla M, Carro M. Effects of exogenous cellulase supplementation on microbial growth and ruminal fermentation of a high-forage diet in Rusitec fermenters. J. Anim. Sci. 2007;85(8):1962-70.

46. Giraldo L, Tejido M, Ranilla M, Ramos S, Carro M. Influence of direct-fed fibrolytic enzymes on diet digestibility and ruminal activity in sheep fed a grass hay-based diet. J. Anim. Sci. 2008;86(7):1617-23.

47. Beauchemin K, Colombatto D, Morgavi D, Yang W. Use of exogenous fibrolytic enzymes to improve feed utilization by ruminants. J. Anim. Sci. 2003;81(14_suppl_2):E37-E47.

48. Ribeiro G, Gonçalves L, Pereira L, Chaves A, Wang Y, Beauchemin K, et al. Effect of fibrolytic enzymes added to a Andropogon gayanus grass silage-concentrate diet on rumen fermentation in batch cultures and the artificial rumen (Rusitec). Animal. 2015;9(7):1153-62.

49. Ribeiro GO, Badhan A, Huang J, Beauchemin KA, Yang W, Wang Y, et al. New recombinant fibrolytic enzymes for improved in vitro ruminal fiber degradability of barley straw. J. Anim. Sci. 2018;96(9):3928-42.

50. Robert C, Bernalier-Donadille A. The cellulolytic microflora of the human colon: evidence of microcrystalline cellulose-degrading bacteria in methane-excreting subjects. FEMS Microbiol Ecol. 2003;46(1):819.

51. Wedekind KJ, Mansfield HR, Montgomery L. Enumeration and isolation of cellulolytic and hemicellulolytic bacteria from human feces. Applied and Environmental Microbiology. 1988;54(6):1530-5.

52. Kopečný J, Hajer J, Mrázek J. Detection of cellulolytic bacteria from the human colon. Folia Microbiol. 2004;49(2):175. 
53. Shetty SA, Marathe NP, Lanjekar V, Ranade D, Shouche YS. Comparative genome analysis of Megasphaera sp. reveals niche specialization and its potential role in the human gut. PloS one. 2013;8(11):e79353.

54. Pustjens AM, de Vries S, Schols HA, Gruppen H, Gerrits WJ, Kabel MA. Understanding carbohydrate structures fermented or resistant to fermentation in broilers fed rapeseed (Brassica napus) meal to evaluate the effect of acid treatment and enzyme addition. Poult Sci. 2014;93(4):926-34.

55. Castillo M, Martín-Orúe S, Anguita M, Pérez J, Gasa J. Adaptation of gut microbiota to corn physical structure and different types of dietary fibre. Livest. Sci. 2007;109(1-3):149-52. 
Summary 
Rapeseed meal (RSM), a byproduct from rapeseed oil production, is not only a suitable protein source for swine feed but also a potential energy source. RSM contains a high amount of cell wall polysaccharides, even higher when compared to soybean meal commonly used in the feed industry. A drawback of RSM is that the complex cell wall polysaccharides cannot be utilized by endogenous enzymes from monogastric animals, and also can only be partly fermented by the microbial community in the gastrointestinal tract (GIT). Therefore, enzymatic and chemical treatment on RSM were performed to improve the recalcitrant fibre degradability of RSM.

Chapter 1 reviews the carbohydrate composition in RSM, and polysaccharide degradation in RSM by microbes. The possible processing methods to increase use of recalcitrant fibres are described as well. In addition, the literature was reviewed on microbiota composition in pigs. At the end, different in vitro fermentation models were introduced in the chapter, including batch fermentation and dynamic fermentation models (TIM-2, SHIME and SIMGI). Chapter 2 describes the development of the Swine Large Intestinal in vitro Model (SLIM), which was developed based on the human, computer-controlled, dynamic TNO in vitro model of the colon, nick-named TIM-2. First, physiological parameters were modified from humans to pigs. Briefly, the temperature was changed from $37{ }^{\circ} \mathrm{C}$ to $39{ }^{\circ} \mathrm{C}$, and the $\mathrm{pH}$ changed from 5.8 to 5.9. Moreover, the simulated ileal efflux medium (SIEM) used to feed the microbiota in the model was modified from humans to pigs (SIEMP).

In Chapter 3, a shot of $5 \mathrm{~g}$ (modified) RSM was directly fed to the standardized swine gut microbiota, and the effect of processing methods on the RSM utilization and microbiota composition was studies in SLIM. The present study clearly demonstrated that both enzymatic and chemical pretreatment on RSM shifted its cell wall polysaccharide structure, subsequently altering microbial community composition and functional profile compared to untreated RSM, and eventually increased fibre degradability as evaluated by SCFA production. Furthermore, glycome profiling showed that the abundance of cell wall polysaccharides were dynamically changed during fermentation, and did not continuously decrease during the fermentation period as one might expect.

Continuous fermentation of the modified RSM for $48 \mathrm{~h}$ in SLIM was described in Chapter 4. The current study demonstrated that microbiota composition was significantly affected by both RSM (processed or not) and adaptation time. Unweighted UniFrac showed that microbial community composition was significantly separated between processed RSM and CON, and CELL and ALK in general changed the microbiota composition more than PECT1 and PECT2 did. Carbohydrate metabolism related microbial functions were predicted to significantly increase after the fibre fermentation period. However, degradability of the processed RSM was not improved compared to CON during the fibre fermentation period, as assessed by SCFA production, which indicated that a relative long adaptation period is needed after a diet change to RSM for swine microbiota.

In Chapter 5, the swine gut microbiota was adapted to (modified) RSM for $48 \mathrm{~h}$ first, which was followed by a shot of $5 \mathrm{~g}$ (modified) RSM as evaluation period. Afterwards, the degradability of (modified) RSM was validated in vivo (ileal cannulated pigs) by the mobile nylon bag technique. CELL and ALK feeding considerably changed the swine microbiota structure and functional profile compared to CON, which did not occur with PECT1 and PECT2. The hypothesis is entertained that this results from the different cell wall architecture of RSM once processed by this carbohydrase or alkaline treatment. The increase in relative abundance of pathways involved in carbohydrate fermentation in the microbiota fed CELLor ALK-treated RSM can be considered as a positive effect of these treatments in fibre 
utilization and SCFA production. Moreover, it was validated in pigs that CELL and ALK feeding improved the overall degradation of RSM by the mobile nylon bag technique. With all the data collectively considered, we speculate that carbohydrase enzyme, i.e. CELL, improve fibre degradation of RSM during the fermentation by changing the microbial community structure and enzymatic activity and subsequently shifting the microbiota metagenomic functional profile.

Chapter 6 describes a preliminary novel high-throughput technique to screen polysaccharide structures. The results showed high reproducibility and specificity in recognizing polysaccharides by the glycoprofiling technique, making use of fluorescently labeled carbohydrate binding modules (CBMs), which are fibre recognition domains of amongst others carbohydrases 
Samenvatting 
Koolzaadmeel (RSM), een bijproduct van de productie van koolzaadolie, is niet alleen een geschikte eiwitbron voor varkensvoer, maar ook een potentiele energiebron. RSM bevat een hoog gehalte aan celwandpolysacchariden, zelfs nog hoger in vergelijking met sojameel dat veel wordt gebruikt in de diervoederindustrie. Een nadeel van RSM is dat de complexe celwandpolysacchariden niet kunnen worden gebruikt door endogene enzymen van monogastrische dieren, en ook slechts gedeeltelijk kunnen worden gefermenteerd door de microbiële gemeenschap in het maagdarmkanaal. Daarom werd een enzymatische en chemische behandeling op RSM uitgevoerd om de weerbarstige vezelafbraak te verbeteren.

Hoofdstuk 1 geeft een overzicht van de koolhydraatsamenstelling in RSM en de afbraak van polysacchariden in RSM door microben. De mogelijke verwerkingsmethode om het gebruik van weerbarstige vezels te vergroten, wordt ook beschreven. Daarnaast werd ook de litearuur samengevat met betrekking tot de samenstelling van de microbiota bij varkens. Uiteindelijk zijn in het hoofdstuk verschillende in vitro fermentatiemodellen geïntroduceerd, waaronder batch fermentatie en dynamische fermentatie modellen (TIM-2, SHIME en SIMGI).

Hoofdstuk 2 beschrijft de ontwikkeling van het Swine Large Intestinal in vitro Model (SLIM), dat is ontwikkeld op basis van het menselijke, computergestuurde, dynamische TNO in vitro model van het colon, met de bijnaam TIM-2. Ten eerste werden fysiologische parameters gewijzigd van mens naar varken. In het kort werd de temperatuur veranderd van $37^{\circ} \mathrm{C}$ naar $39^{\circ} \mathrm{C}$, en de $\mathrm{pH}$ van 5,8 naar 5,9. Tevens werd het gesimuleerde ileale effluxmedium (SIEM), dat wordt gebruikt om de microbiota in het model te voeden, gewijzigd van mens tot varken (SIEMP).

In Hoofdstuk 3 werd een shot van 5 g (gemodificeerde) RSM direct aan de gestandaardiseerde darmmicrobiota toegevoerd, en het effect van verwerkingsmethoden op het gebruik van RSM en de samenstelling van de microbiota werd bestudeerd in SLIM. De huidige studie toonde duidelijk aan dat zowel enzymatische als chemische voorbehandeling op RSM de polysaccharidestructuur van de celwand verschoof, waardoor de microbiële gemeenschapssamenstelling en het functionele profiel veranderden in vergelijking met onbehandeld RSM, en uiteindelijk de afbreekbaarheid van vezels verhoogde, zoals beoordeeld door korte-keten vetzuur-productie. Bovendien toonde glycome-profilering aan dat de samenstelling van de celwandpolysacchariden tijdens de fermentatie dynamisch veranderde en niet continu afnam tijdens de fermentatieperiode, zoals men zou verwachten. Continue fermentatie van de gemodificeerde RSM gedurende 48 uur in SLIM is beschreven in Hoofdstuk 4. De huidige studie toonde aan dat de samenstelling van microbiota significant werd beïnvloed door zowel RSM (al dan niet geprocessed) als fermentatietijd. Ongewogen UniFrac toonde aan dat de samenstelling van microbiële gemeenschappen significant gescheiden was tussen verwerkte RSM en CON, en CELL en ALK veranderden in het algemeen de samenstelling van de microbiota meer dan PECT1 en PECT2. Aan koolhydraatmetabolisme gerelateerde microbiële functies waren significant verhoogd na de vezelfermentatieperiode. De afbreekbaarheid van het verwerkte RSM was echter niet verbeterd in vergelijking met $\mathrm{CON}$ tijdens de vezelfermentatieperiode, zoals beoordeeld door korte-keten vetzuur-productie, wat erop wees dat een relatief lange aanpassingsperiode nodig is voor microbiota van varkens na een dieetwijziging naar RSM.

In Hoofdstuk 5 werd de microbiota van de darm van de varkens eerst gedurende 48 uur aangepast aan (gemodificeerd) RSM, gevolgd door een inname van $5 \mathrm{~g}$ (gemodificeerd) RSM als evaluatieperiode. Daarna werd de afbreekbaarheid van (gemodificeerd) RSM gevalideerd in een in vivo studie (gecanuleerde varkens) door middel van een mobiele nylon zaktechniek. CELL- en ALK-voeding veranderden de microbiota-structuur en het functionele profiel van 
varkens aanzienlijk in vergelijking met CON, wat niet voorkwam bij PECT1 en PECT2. De hypothese wordt verondersteld dat dit het gevolg is van de verschillende celwandarchitectuur van RSM, eenmaal verwerkt door deze carbohydrase of alkaline behandeling. De toename van de relatieve overvloed aan microbiele routes die gepaard gaan met koolhydraatfermentatie in CELL of ALK kan worden beschouwd als een positief effect van deze behandelingen bij vezelgebruik en korte-keten vetzuur-productie. Bovendien werd bij varkens gevalideerd dat CELL- en ALK-behandeling de algehele afbraak van RSM verbeterde met behulp van de mobiele nylon zaktechniek. Alle gegevens gezamenlijk beschouwd, speculeren we dat het carbohydrase-enzym, d.w.z. CELL, de afbraak van vezels van RSM tijdens de fermentatie verbetert door de microbiële gemeenschapsstructuur en enzymatische activiteit te veranderen en vervolgens het metagenomische functionele profiel van de microbiota te veranderen.

Hoofdstuk 6 beschrijft een niet volledige nieuwe high-throughput techniek om polysaccharidestructuren te screenen. De resultaten toonden een hoge reproduceerbaarheid en specificiteit aan bij het herkennen van polysacchariden door de glycoprofileringstechniek, gebruikmakend van fluorescent gelabelde koolhydraatbindende modules (CBM's), wat vezelherkenningsdomeinen van onder andere koolhydraat hydrolyserende enzymen zijn. 
-

总结

- 
菜籽粕--生产菜籽油的副产品, 不仅是合适的猪的蛋白质饲料原料, 而且还是潜在的 能源饲料原料。菜籽粕含有大量的细胞壁多糖, 与饲料企业中通常使用的豆粕相比, 非淀粉多糖含量甚至更高。使用菜籽粕作为饲料原料的一个缺点是复杂的细胞壁多 糖不能被单胃动物的内源酶所利用, 也只能被肠道中的微生物群落部分发酵。因此, 本研究对菜籽粕进行了酶和化学处理, 以改善菜籽粕中纤维的降解性。

第 1 章回顾了菜䊏粕中的碳水化合物的组成以及微生物对菜籽粕中多糖的降解。还描 述了提高纤维降解率的可能的加工方法。另外, 文献回顾了猪的微生物菌落组成。 最后, 本章介绍了不同的体外发酵模型（TIM-2, SHIME 和 SIMGI）。

第 2 章介绍了猪大肠体外模型（SLIM）的开发, 该模型是基于人的, 全电脑控制的, 结肠体外模型 (昵称为 TIM-2) 开发的。首先, 将人的生理参数改为猪的生理参数。 即, 温度从 $37^{\circ} \mathrm{C}$ 改变为 $39^{\circ} \mathrm{C}$, 并且 $\mathrm{pH}$ 从 5.8 改变为 5.9 。此外, 微生物的培养基已 从人的 (SIEM) 开发为猪的 (SIEMP)。

在第 3 章中, 将 5 克处理过的菜籽粕添加到标准化的猪肠道菌群中, 并在 SLIM 中研 究了加工方法对菜籽粕的利用率和菌群组成的影响。本研究清楚地表明, 对菜籽粕 的酶制剂和化学预处理均改变了其细胞壁多糖结构, 与未处理的菜籽粕相比, 其改 变了微生物群落组成和功能特性, 最终提高了纤维的降解率 (通过短链脂肪酸

(SCFA) 的产量评估)。此外, 多糖组成分析表明, 发酵过程中细胞壁多糖的含量 是动态变化的, 并且在发酵过程中并没有如人们所预期的那样持续减少。

加工的菜籽粕在 SLIM 中连续发酵 48 小时的研究在第 4 章中进行了描述。当前研究 表明, 微生物群组成受已加工或未加工的菜籽粕和发酵时间的影响很大。未加权的 UniFrac 分析表明, 经过处理的菜籽粕组和对照组之间的微生物群落组成差异显著, 并且 CELL 和 ALK 比 PECT1 和 PECT2 更能改变微生物群落组成。纤维发酵后, 与 碳水化合物代谢相关的微生物功能显着增加。但是, 通过 SCFA 产量评估, 在纤维发 酵期间, 与对照组相比, 加工后的菜籽粕的降解率并未得到改善, 这表明, 饲料原 料改为菜籽粕后, 猪的微生物菌群需要相对较长的适应期。

在第 5 章中, 猪肠道菌群首先适应加工后的菜籽粕 48 小时, 然后添加 5 克该菜籽粕 作为试验期。然后, 通过猪的瘘管技术, 验证了加工处理确实提高了菜籽粕的降解 性。与对照组相比, CELL 和 ALK 喂养大大改变了猪的微生物菌群结构和功能, 而 PECT1 和 PECT2 则没有。因此我们提出了这样的假设: 这是由于通过该酶制剂或碱 处理改变了菜籽粕的细胞壁结构所致。与碳水化合物利用相关的微生物丰度的增加 对纤维的利用和 SCFA 的产生有积极作用。此外, 通过移动尼龙袋技术证实在猪饲料 中使用 CELL 和 ALK 可改善菜籽粕的降解率。综合考虑所有数据, 我们推测, 通过 改变微生物群落结构和酶活性并随后改变微生物菌落的宏基因组功能谱, 纤维降解 酶制剂 (即 CELL) 可提高菜籽粕中的纤维降解率。

第 6 章介绍了分析多糖结构的高通量技术。结果显示, 通过使用荧光标记的碳水化合 物结合模块 (carbohydrate binding modules), 该技术在糖基分析中具有很高的重现性 和特异性。 


\section{Impact}


It is complicated and costly to investigate the effect of feed fermentation by the gut microbiota in pigs in vivo, due to the interaction with other feed-components (such as other fibers) in the swine feed, the ethical considerations (such as using pathogens, toxic compounds, and animal experiments itself) and other uncontrollable factors (such as infectious disease or death during the experiment), which are important for reproducibility of results. In vitro fermentative models are considered excellent alternatives to study the effect on the composition and/or activity of the gut microbiota without ethical constraints. They allow the reasonable high throughput screening of a large number of substances such as dietary ingredients and components thereof such as enzyme-, chemical- or physicallytreated recalcitrant fibres. In this thesis, a Swine Large Intestinal in vitro Model (SLIM) was developed, which was developed based on the human, computer-controlled, dynamic TNO in vitro model of the colon, nick-named TIM-2. Therefore, the new developed SLIM can be used by researchers in both universities and feed-related companies to study the scientific questions which cannot be answered in vivo as mentioned previously. In this thesis, the SLIM system was used to study the (increased) fermentation of (enzyme- or chemically-processed) recalcitrant fibres in rapeseed meal (RSM), with the aim to increase energy extraction from the diet, through liberation of short-chain fatty acids (SCFA) produced by the pig gut microbiota.

The European Union (EU) is the second-largest importer of raw material for feed (especially protein-rich feed ingredients) in the world, after China. Therefore, in order to have a more sustainable supply of responsible protein-rich ingredients, the European livestock sector needs an alternative local protein feed ingredient to fill the "protein gap". Rapeseed meal (RSM), a byproduct from rapeseed oil production, is not only a suitable protein source for animal feed but also a potential energy source. RSM contains a high amount of cell wall polysaccharides, even higher when compared to soybean meal commonly used in the feed industry. However, the high amount of pectins in RSM can also reduce the absorption of other nutrients in the gut. Therefore, the aim of the project was to improve utilization of recalcitrant fibre of by-product of rapeseed oil in pigs. Enzymatic and chemical treatment on the RSM were performed in the project to improve the digestibility and fermentability of these by-product in pigs.

The current thesis provides the first detailed analysis of changes in the swine intestinal microbiota due to RSM processed by enzymatic and chemical treatment using the newly developed SLIM sytem. The present studies clearly demonstrated that both enzymatic (cellulase or two different pectinases) and chemical (6 N sodium hydroxide; alkaline) pretreatment on RSM shifted its cell wall polysaccharide structure, subsequently altering microbial community composition and functional profile compared to untreated RSM, and eventually increased fibre degradability as evaluated by SCFA production in SLIM. Moreover, it was validated in pigs by the mobile nylon bag technique that cellulase and alkaline treatment on RSM improved the overall degradation of RSM. Our findings that the specific treatments increased fibre degradation in RSM could help to guide feed additive strategies to improve efficiency and productivity in swine industry. The current study gave insight into how feed enzymes may modulate microbial status, which provides good opportunity to develop novel carbohydrase, particularly in swine feed. In particular, the genomes of microorganisms that were stimulated by the processed RSM can be mined for novel carbohydrases, that may be used as novel enzymes to pretreat RSM to increase the use of its recalcitrant fibres by the pig gut microbiota.

In order to develop new methods to improve the degradability of plant cell wall 
polysaccharides, a better understanding of the cell wall polysaccharide composition and structure and their interactions are essential. In the thesis, a high-throughput technique was developed to screen composition and structure of plant cell wall polysaccharides. The technique (glycome profiling of plant cell wall polysaccharides, or glycoprofiling) was developed with the use of carbohydrate binding modules (CBMs) with unique specificities for plant cell wall polysaccharides, linked to a green fluorescent protein (GFP) as reporter. This toolbox may become essential in the study of the dynamics of breakdown of dietary fibres by the (pig) gut microbiota.

Researchers from both academia and industry can access our results from scientific journals. The result of SLIM model is published in Beneficial Microbes, the results of RSM degradation in pigs are published in Journal of Agricultural and Food Chemistry and Frontiers in Microbiology, and the results of the glycoprofiling technique are prepared for publication. The results call for additional research into recalcitrant fibre fermentation by the (pig) gut microbiota, e.g., by combining multiple enzymes for pretreatment. Both tools (SLIM and glycoprofiling) in our view are pivotal for studying the increased use of feed components by the gut microbiota, leading to increased energy extraction for the host. The use of the tools can be extended to other fibre-sources and other hosts (beyond pigs). 
Impact 
Acknowledgments 
Doing $\mathrm{PhD}$ is a kind of "practice Buddhism", therefore, I want to express my greatest gratitude to the people who inspired, helped, and/or was willing to help me during the journey.

First of all, Koen, thank you for supervising me and for helping me solve problems during my $\mathrm{PhD}$. Whenever I had a trouble, after I went to your office, you always gave me a suitable solution and I left with a happy face. You are definitely a "problem terminator". Thank you for all the critical questions and thoughtful thinking on my articles and thesis.

Thanks Dr. Sonja de Vries from Wageningen University. Thanks for hosting me in the Group of Animal Nutrition. Thanks for helping me collecting pig feces. Thank you for the assistance during the nylon bag experiment. Thank you for your critical comments when reviewing my manuscripts. And thanks to all the people from the Group of Animal Nutrition who helped me during this period.

I would like to thank Prof. Dr Henk Schols from Wageningen University. Even though I still do not know you are my official co-promotor or not, you are definitely the other promotor of mine in my heart. Thank you for hosting me in you lab, which let me feel like home. Thank you for sharing your knowledge of carbohydrate to me, from which I learnt a lot. Thank you for all the critical comments when reviewing my manuscript. Christiane, thank you for the technical assistance of using GC, HPSEC and HPAEC. And thanks to all the people from the Laboratory of Food Chemistry who helped me during this period.

Thanks Rogier, Nicole and all other support staffs in campus Venlo, thanks for your assistant during my $\mathrm{PhD}$ period.

I also would like to specially thank my colleagues in Villa Flora at Maastricht University. Thank Dr Carlota Bussolo de Souza and all the technicians-Rob, Sanne and Jessica. Thanks for all the technical assistance you provided during the past several years. Thank Carlota for teaching me to use TIM-2. Thank Jessica for organizing everything very well in the lab. I would like to specially thank Sanne for helping me with the CBM work. I would like to thank Rob for sharing his experience how to handle marriage, the "yes" thing. Thank all the colleagues, whom were not only mentioned on this paragraph but also previous and latter paragraphs in Villa Flora, for the gift cards and investment on my little Ran.

Thank all my office mates and colleagues, Evy, Miram, Monica, Britt, Anouk, Tim, and Ardi. Thank you for kindly and patiently explaining Dutch culture things to me. Thanks for the activities you organized, I really enjoyed them. I would like to specially thank Evy for helping with calling my family doctor, oxxio, tax office, and so many other things I can't remember now. Dank je wel!

Thanks all the visiting PhDs from worldwide, Maria1, Maria 2, Diogo, Lucia, Bea, Don, Stephanie, Paulina and GIZEM!! Because of you guys, I felt not alone in the Netherlands. Thank Lucia and Bea for teaching me Spanish. You are my amiga. Thank Bea for inviting me to Efteling. It was amazing, even though I was scared of the roll-coaster. I rode twice in the front line with you!! I would like to thank Gizem for your help in the lab, and also the special gift for Ran from your mom, which was all the way from Turkey and made by your mom herself. My wife and I really like it, and of course, Ran herself likes it too (I guess). 
Thank all my friends in Wageningen, Taojun, Caifang, An Ran and Zhang Chen, and other PhDs I do not know your names. Thanks for hosting me and feeding me with your best food during my research in Wageningen. I am really missing the hot-pot now. One day I wish we can unite again in China.

I also would like to thank all the Chinese friends in Venlo and Maastricht, Nemo, Jim, Vicky, Gloria, Xie Chen, Qiao jie, Lily, Mutong, Guo Le, and others. Thank you for the activities and food. We had a very nice time in Venlo and Maastricht.

最后, 感谢我的爸爸和妈妈, 谢谢你们对我无条件的关爱与支持。感谢姐姐和姐夫 对我的支持和替我照顾好了爸妈。感谢小燕子和坨坨哥的理解与爱。感谢坨坨哥的 到来，你是我最好的“博士论文”。

Thank you!

Dank ye wel!

谢谢!

Cheng 
About the author 


\section{Curriculum Vitae}

Cheng Long was born on the $19^{\text {th }}$ of July 1990 in Pingxiang, China. In 2009, he started his bachelor in Business Administration at Jiangxi Agricultural University, after one semester, and he decided to study Animal Science. In 2013, he completed his bachelor in Animal Science. From here, he went on further, to pursue his master in Animal Nutrition and Feed Science at Chinese Academy of Agricultural Sciences where he worked on

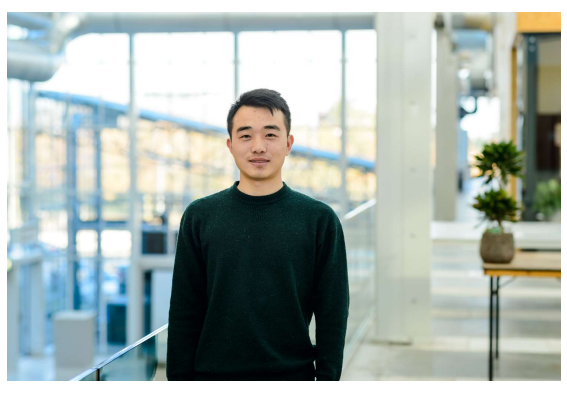
improving egg flavor by inspecting cecal bacteria and trimethylamine concentration when laying hens were administrated rapeseed meal based feed. In September 2016, he started his $\mathrm{PhD}$ at Center for Healthy Eating \& Food Innovation, Maastricht University. His PhD project was "Improving intestinal degradation of recalcitrant fiber in pigs", where he was working on illuminating the characterization of the inaccessible cell wall architecture and the effect of modified fibres on swine gut microbiota. During this project, he also closely cooperated with Group of Animal Nutrition and Laboratory of Food Chemistry at Wageningen University. The outcome of his research during his $\mathrm{PhD}$ is presented in this thesis. 


\section{List of Publications}

Long C, de Vries S, Venema K. Polysaccharides source alters molecular ecological network interaction, functional profile, and short-chain fatty acid production in a porcine gut microbiota. Beneficial Microbes. 2020:11 (6), 591-610. DOI: 10.3920/BM2020.0006.

Long C, de Vries S, Venema K. Differently Pre-treated Rapeseed Meals Affect in vitro Swine Gut Microbiota Composition. Frontiers in Microbiology. 2020;11(2125). DOI: 10.3389/fmicb.2020.570985.

Long C, Rösch C, de Vries Sonja, Schols HA, Venema K. Cellulase and alkaline treatment rather than pectinase improves intestinal microbial degradation of recalcitrant fibres of rapeseed meal in pigs. Journal of Agricutrual and Food Chemistry. 2020: 68(39) 1101111025. DOI: $10.1021 /$ acs.jafc.0c03618

Long, C, Venema K. Pretreatment of rapeseed meal increases its recalcitrant fibre fermentation and alters the microbial community in an in vitro model of swine large intestine. Frontiers in Microbiology. 2020, DOI: 10.3389/fmicb.2020.588264.

Long, C, Venema K. Chemical and nutritional characteristics, and microbial degradation of rapeseed meal recalcitrant carbohydrates. Submitted for publication.

Long, C, Venema K. High-throughput screening of structures of plant cell wall polysaccharides using a novel method. Prepared for publication.

Other work:

Long, C., Wang, J., Zhang, H.J., Wu, S.G. and Qi, G.H., 2017. Effects of dietary rapeseed meal supplementation on cecal microbiota in laying hens with different flavin-containing monooxygenase 3 genotypes. Poultry science, 96(6), pp.1748-1758.

Long C, Chen L, Wang J, Wu SG, Zhang HJ, Yue HY, Qi GH. Effects of Dietary Canola Meal Level on Performance, Egg Quality, Trimethylamine Content in Egg Yolk of FlavinContaining Monooxygenases 3 Genotype Laying Hens[J]. Chinese Journal of Animal Nutrition, 2016, 28(6): 1687-1695.

Long C, Wang J, Wu SG, Zhang HJ, Yue HY, Qi GH. Influence of Feed Factors on Egg Flavor and the Measures for Flavor Improvement [J]. Chinese Journal of Animal Nutrition, 2015, 27(2):352-358.

Wang, J., Long, C., Zhang, H., Zhang, Y., Wang, H., Yue, H., Wang, X., Wu, S. and Qi, G., 2016. Genetic variant in Flavin-containing monooxygenase 3 alters lipid metabolism in laying hens in a diet-specific manner. International journal of biological sciences, 12(11), p.1382.

Wang, J., Zhang, H.J., Xu, L., Long, C., Samuel, K.G., Yue, H.Y., Sun, L.L., Wu, S.G. and Qi, G.H., 2016. Dietary supplementation of pyrroloquinoline quinone disodium protects 
against oxidative stress and liver damage in laying hens fed an oxidized sunflower oil-added diet. animal, 10(7), pp.1129-1136.

Wang, J., Zhang, H.J., Samuel, K.G., Long, C., Wu, S.G., Yue, H.Y., Sun, L.L. and Qi, G.H., 2015. Effects of dietary pyrroloquinoline quinone disodium on growth, carcass characteristics, redox status, and mitochondria metabolism in broilers. Poultry Science, 94(2), pp.215-225. 
Overview of completed training activities

\section{Discipline specific courses}

\section{Courses}

The Intestinal Microbiome and Diet in Human and Animal Health, Wageningen, The Netherlands, 2017

$15^{\text {th }}$ European Training Course on Carbohydrates, Wageningen, The Netherlands, 2018

Gut health in pigs and poultry, Wageningen, The Netherlands, 2019

\section{Conferences}

The $6^{\text {th }}$ Beneficial Microbes Conference, Amsterdam, The Netherlands, 2017

The $19^{\text {th }}$ Gutday, Venlo, The Netherlands, 2017

$14^{\text {th }}$ International Symposium Digestive Physiology of Pigs, Brisbane, Australia, 2018

The $7^{\text {th }}$ Beneficial Microbes Conference, Amsterdam, The Netherlands, 2018

The $20^{\text {th }}$ Gutday, Middleburg, The Netherlands, 2018

\section{General courses}

Effective communication Intermediate, Maastricht, The Netherlands, 2017

Biosafety in the laboratory, Maastricht, The Netherlands, 2018 